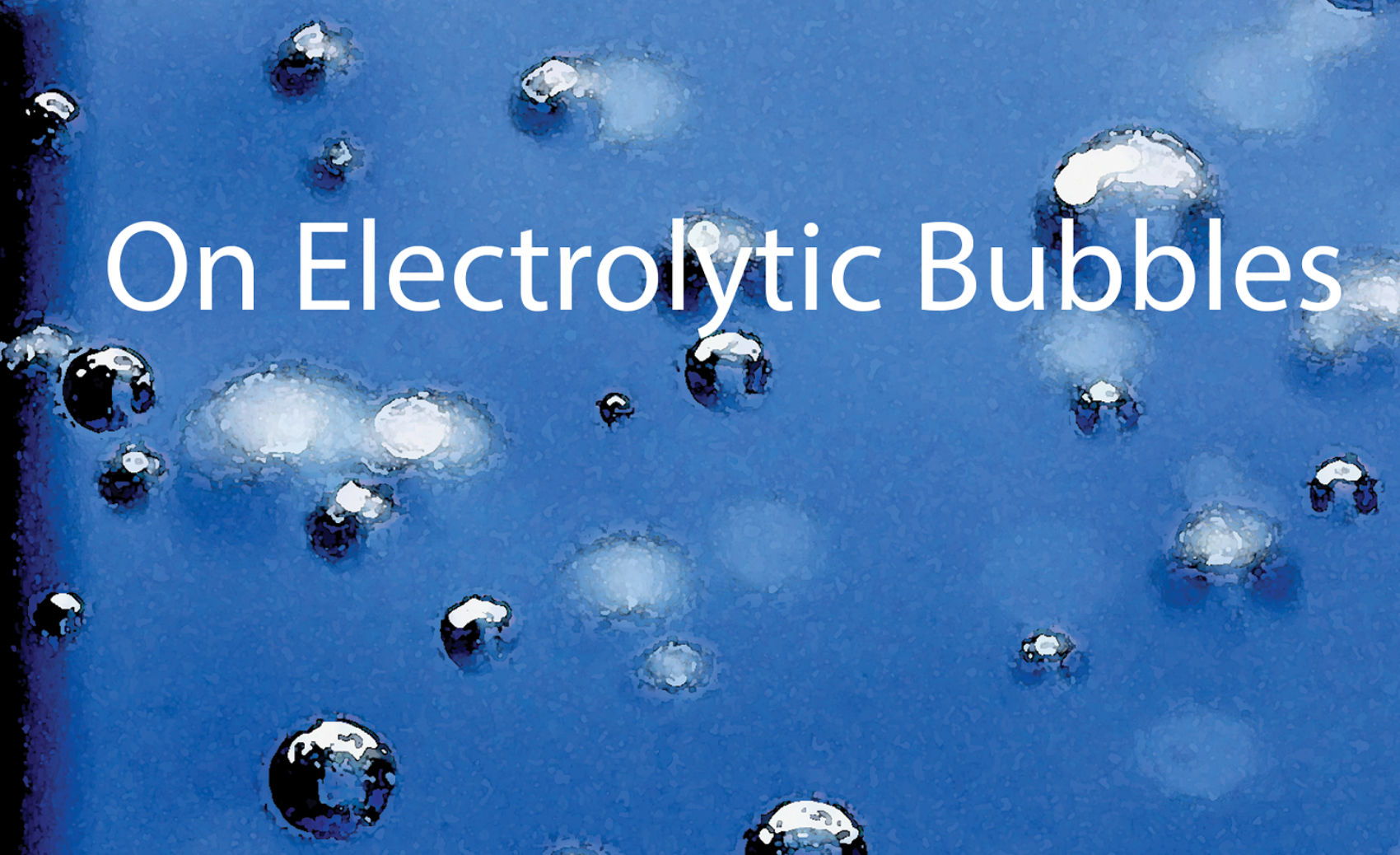

$\frac{6}{2}$

5
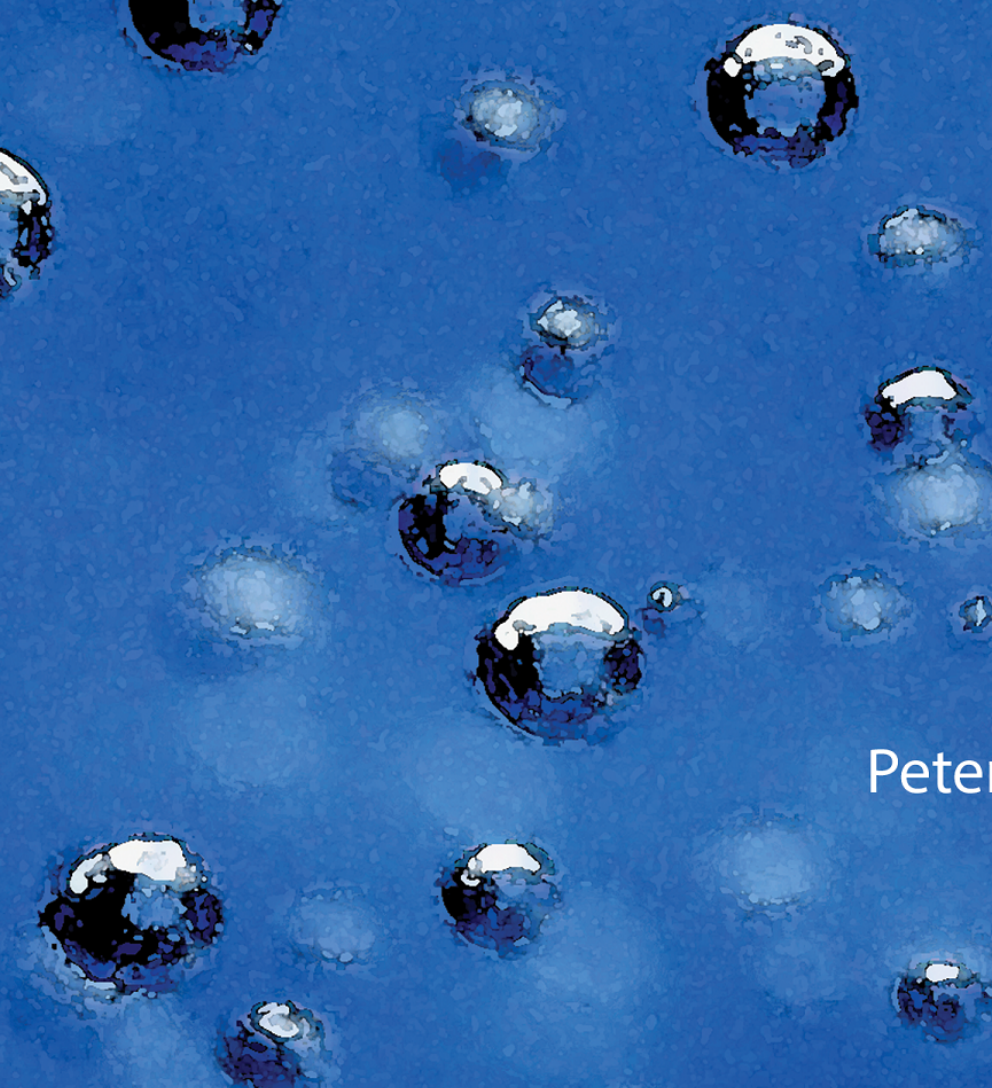


\section{ON ELECTROLYTIC BUBBLES}

Peter van der Linde 



\section{ON ELECTROLYTIC BUBBLES}

\section{DISSERTATION}

to obtain

the degree of doctor at the University of Twente, on the authority of the rector magnificus, prof.dr. T.T.M. Palstra, on account of the decision of the Doctorate Board, to be publicly defended on Thursday the $4^{\text {th }}$ of April 2019 at 12:45 hours

by

\section{Peter van der Linde}

born on the $5^{\text {th }}$ of August 1985

in Maartensdijk, the Netherlands 
This dissertation has been approved by:

Supervisor:

prof. dr. J.G.E. Gardeniers

Co-supervisor:

dr. ir. D. Fernández Rivas

The work in this thesis was carried out at the Mesoscale Chemical Systems group of the Faculty of Science and technology, and the MESA+ Institute for Nanotechnology, both at the University of Twente. This work was supported by the Netherlands Centre for Multiscale Catalytic Energy Conversion (MCEC), an NWO Gravitation programme funded by the Ministry of Education, Culture and Science of the government of the Netherlands.

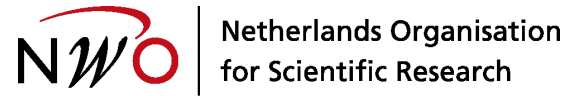

Nederlandse titel: Elektrolytische bellen

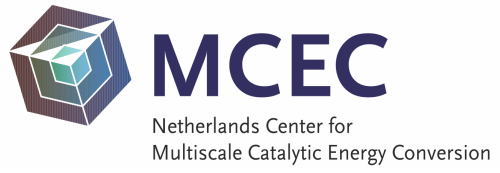

Publisher:

Peter van der Linde, Mescoscale Chemical Systems, University of Twente, P.O. Box 217, 7500AE Enschede, The Netherlands

p.vanderlinde@utwente.nl / petervanderlinde.email@gmail.com

Cover design. Front: image "Bubbles" (CC0 1.0) adapted to a watercolor. Back: scanning electron microscopy image of crystals on a silicon substrate. Printed by: Ipskamp Printing

Lay-out: Peter van der Linde

ISBN: 978-90-365-4741-3

DOI: $10.3990 / 1.9789036547413$

(C) 2019 Enschede, The Netherlands. All rights reserved. No parts of this thesis may be reproduced, stored in a retrieval system or transmitted in any form or by any means without permission of the author. Alle rechten voorbehouden. Niets uit deze uitgave mag worden vermenigvuldigd, in enige vorm of op enige wijze, zonder voorafgaande schriftelijke toestemming van de auteur. 


\section{GRADUATION COMMITTEE:}

Chairman prof. dr. J.L. Herek

Supervisor prof. dr. J.G.E. Gardeniers Universiteit Twente, TNW

Co-supervisor dr. ir. D. Fernández Rivas Universiteit Twente, TNW

Members prof. dr. ir. N.G. Deen TU Eindhoven

prof. dr. ir. J. Huskens Universiteit Twente, TNW

prof. dr. P.E. de Jongh Universiteit Utrecht

prof. dr. D. van der Meer Universiteit Twente, TNW

prof. dr. G. Mul Universiteit Twente, TNW 


\section{Contents}

1 Introduction 1

1.1 Hydrogen as energy carrier . . . . . . . . . . . 2

1.2 The aim of this work . . . . . . . . . . 5

1.3 Thesis structure . . . . . . . . . . . . . 7

2 Influence of bubbles on energy and mass transfer efficiencies $\begin{array}{ll}\text { of electrochemical systems } & 9\end{array}$

2.1 Introduction . . . . . . . . . . . . . . 10

2.2 Hydrogen gas to replace fossil fuels . . . . . . . . . . . . 11

2.3 Electrolysis at the mesoscale . . . . . . . . . . . . . 12

2.4 Overpotential losses . . . . . . . . . . . . . . . 13

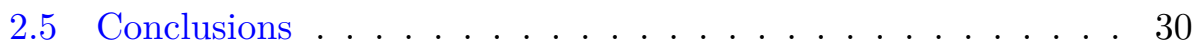

3 Gas bubble evolution on microstructured silicon substrates 33 3.1 Introduction . . . . . . . . . . . . . . . 34

3.2 Materials and methods . . . . . . . . . . . . . . 36

3.3 Results and discussion . . . . . . . . . . . . . . . . . . 39

3.4 Conclusions . . . . . . . . . . . . . . . . 54

4 Electrolysis-driven and pressure-controlled diffusive growth of successive bubbles on micro-structured surfaces $\quad 61$

4.1 Introduction . . . . . . . . . . . . . . . . . . 62

4.2 Experiments . . . . . . . . . . . . . . 6 65

4.3 Results and discussion . . . . . . . . . . . . . . 72

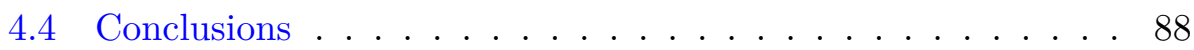

5 Spatial control over electrolytic bubbles nearby gas evolving $\begin{array}{ll}\text { electrodes } & 101\end{array}$

5.1 Introduction . . . . . . . . . . . . . . . . . . 102

5.2 Experimental . . . . . . . . . . . . . . . . . . 103

5.3 Results and Discussion . . . . . . . . . . . . . . . . . 104

5.4 Summary . . . . . . . . . . . . . . . . . . . . 110 
6 Perspectives for future studies and conclusions $\quad 115$

6.1 Introduction . . . . . . . . . . . . . . 116

6.2 Electrolyzers with multiple nucleation sites . . . . . . 116

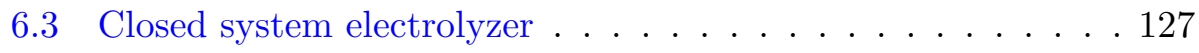

6.4 Final conclusions . . . . . . . . . . . . . . . . . . 134

$\begin{array}{lll}7 & \text { Bibliography } & 135\end{array}$

$\begin{array}{ll}\text { Summary } & 153\end{array}$

$\begin{array}{ll}\text { Samenvatting } & 155\end{array}$

$\begin{array}{ll}\text { Publications and presentations } & 157\end{array}$

$\begin{array}{ll}\text { Acknowledgements } & 159\end{array}$

$\begin{array}{ll}\text { About the author } & 161\end{array}$ 


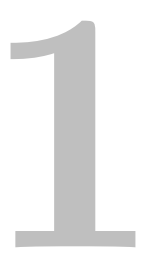

\section{Introduction}

This chapter provides the introduction to the thesis. The later chapters all have individual introductions, specifically providing the introduction for that chapter. In this chapter, the motivation, background, and aim of this work are given. This is finalized by an outline for this thesis where descriptions of the later chapters are given. 


\subsection{Hydrogen as energy carrier}

The natural gas and oil resources readily accessible are limited and a smaller supply than the demand is expected in several decades [1]. Therefore, renewable energy sources have been suggested to relieve the energy demand of these resources [2]. Earth's largest source of renewable energy is sunlight. About 100,000 - 173,000 terawatts are provided by the sun each year to the Earth's surface, in contrast the power consumption is currently about 10 - 20 terawatts annually $[3,4,5]$. Because of the intermittency in both solar energy supply and global demand, energy storage and distribution is required [6]. Solar-driven water splitting is one of the methods that can address the storage of energy and distribution requirements.

During solar-driven water splitting, sunlight is used to convert water to oxygen and hydrogen gas. Hydrogen gas is a dense lightweight energy carrier and can be stored using existing methods of compression. When required, hydrogen gas can be recombined with oxygen gas, either from storage or from environmental air, and upon combustion, energy is released together with water. The fact that no $\mathrm{CO}_{2}$ is emitted during this reaction contributes to another societal issue: $\mathrm{CO}_{2}$ emission has been linked to climate change [7] and therefore renewable energy techniques without $\mathrm{CO}_{2}$ emission are urgently needed.

Due to the favorable band gap of semiconductor materials, they can be used in solar-driven water splitting reactions as photo-electrode materials to absorb photons of incident solar light [8]. Figure 1.1(A) shows a schematic overview of an electrolysis cell with a photo-cathode driving a water splitting reaction. In the semiconductor's valence band, shown in Figure 1.1(B), electrons are promoted to the conduction band with the energy from the absorbed photons. The process leaves behind an electron-hole. This charge separation can be used to create potentials large enough to break water molecules and form oxygen and hydrogen gas. 

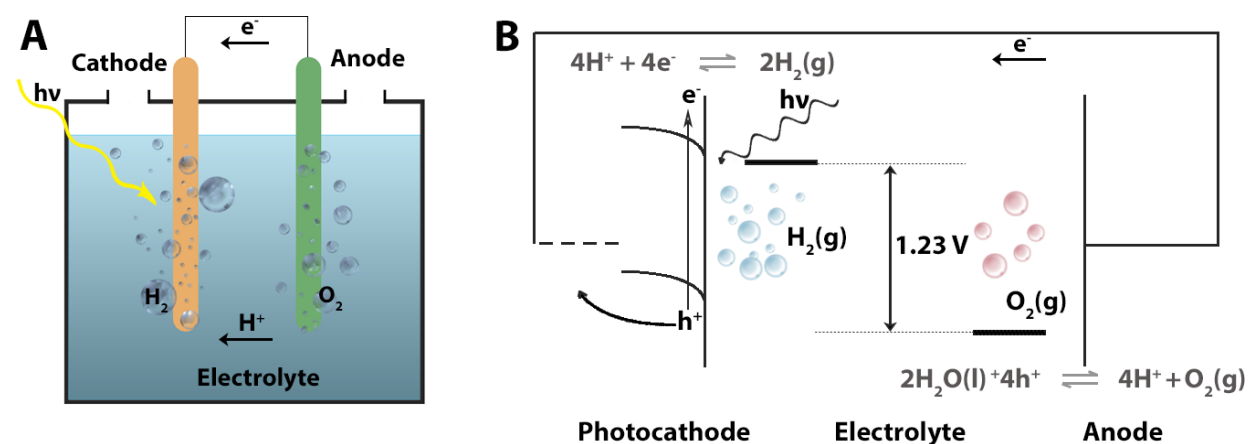

Figure 1.1: A) Electrolysis cell with a photo-active cathode and anode partially submerged in an electrolyte. B) Energy diagram of photo-cathode and metal anode. The electron promotion by absorbed photons leaves behind an electron hole, this is shown at the photo-cathode. The reduction and oxidation half reactions are depicted at the relevant electrodes. 


\subsubsection{Thermodynamic considerations}

During hydrolysis under acidic conditions, the reduction reaction at the cathode is:

$$
2 \mathrm{H}^{+}(a q)+2 e^{-} \rightarrow H_{2}(g) .
$$

While at the anode the oxidation reaction is:

$$
2 \mathrm{H}_{2} \mathrm{O}(l) \rightarrow \mathrm{O}_{2}(g)+4 H^{+}(a q)+4 e^{-} .
$$

In theory, at $\mathrm{pH} 0$ and at standard temperature and pressure, the standard potential for pure water splitting reactions is $-1.23 \mathrm{~V}$. However, in practice a higher potential is required. Entropy, activation energy, mobility of species, concentration of species, impurities of materials, and circuit resistances cause the requirements for the reaction potential to increase. The potential difference between the thermodynamically determined reaction potential and the experimentally observed potential is termed the total overpotential and can be defined as

$$
\eta_{\text {total }}=\eta_{a}+\eta_{\Omega}+\eta_{c}
$$

with $\eta_{a}$ the activation overpotential, $\eta_{\Omega}$ the resistance overpotential, and $\eta_{c}$ the concentration overpotential [9]. Bubbles affect the total overpotential, increase the resistive overpotential, scatter incoming light, and block electrode surfaces needed for water splitting.

\subsubsection{Bubble formation during electrolysis}

Electrolysis of water was first researched by the Dutchmen Paets van Troostwijk and Deiman in 1789 [10]. Using electrogenerated sparks, they decomposed water into hydrogen and oxygen gases. The gases where recombined in a second phase of their experiments via combustion, to provide insight in the composition of the gas mixture. Soon after, in 1800, Nicholson and Carlisle made use of Voltaic piles for the electrolysis of water. Figure 1.2 shows an electrolysis setup from 1800 that made use of a Voltaic pile [11].

Later, in 1834 the first law of electrochemistry was articulated by Faraday [12]. This law states that the mass of a substance liberated at an electrode is proportional to the electric charge used:

$$
m=\left(\frac{Q}{F}\right)\left(\frac{M}{z}\right)
$$

with $m$ the mass in $\mathrm{g}, Q$ the electric charge in C, $F=96485 \mathrm{C} / \mathrm{mol}$ Faradays constant, $M$ the molar mass of the substance in $\mathrm{g} / \mathrm{mol}$, and $z$ the electron valency. 
More recently, the bubble evolution, that is the nucleation, growth and detachment processes of bubbles have been studied in more detail [13, 14, $15]$.

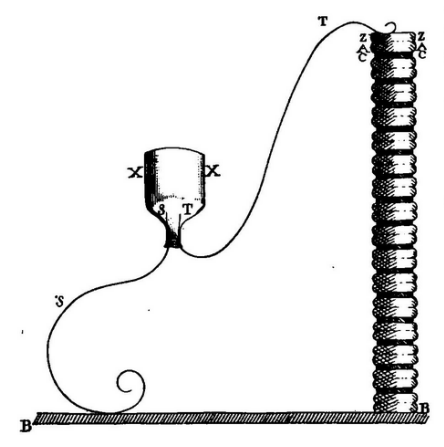

Figure 1.2: Landriani's electrolysis setup in 1800. On the right hand side the voltaic pile is shown, connected via wires $\mathrm{T}$ and $\mathrm{S}$ to a reservoir $\mathrm{X}$. Image reprinted from W. Ostwald. Elektrochemie, Ihre Geschichte un Lehre. Verlag von Veit \& Comp., Leipzig, 1896 [11].

\subsection{The aim of this work}

Figure 1.3 illustrates the general context of this work. The market and the hydrogen economy, a proposed system of energy delivery based on hydrogen, are interrelated and here indicated by the red rectangle. There are alternatives to the hydrogen economy such as the methanol and ethanol economy concepts and nonrenewable energy economies based on coal and nuclear energy. The choice on which direction, or combination of directions is chosen, is up to the decision-makers on a global level and does not concern this work. Here hydrolysis, an important part in the hydrogen economy, and its efficiency is the point of focus, indicated by the green rectangle.

Phenomena occurring in hydrolysis such as the evolution of electrolytic bubbles, a subset of bubbles that are formed as a result of an electrochemical reaction, can occur in other electrolysis processes in which gases form in a liquid. Such processes might be able to benefit from the fundamental knowledge of hydrolysis. In the blue rectangle, two examples of such processes are given. The energy efficiency which we define as the energy requirement to perform hydrolysis, is determined by the total overpotential of the hydrolysis system, of which the activation overpotential component is determined by the reaction kinetics and is therefore intrinsically coupled to the catalyst and the conditions applied during the reaction. In this work we make use of surfaces which may be 
considered catalytic such as platinum electrodes. However, the material choice was based upon chemical inertness of the material and simplicity of use rather than its catalytic activity.

Our goal is to elucidate the phenomena associated to bubble evolution and the electric potential response caused by electrolytic bubbles.

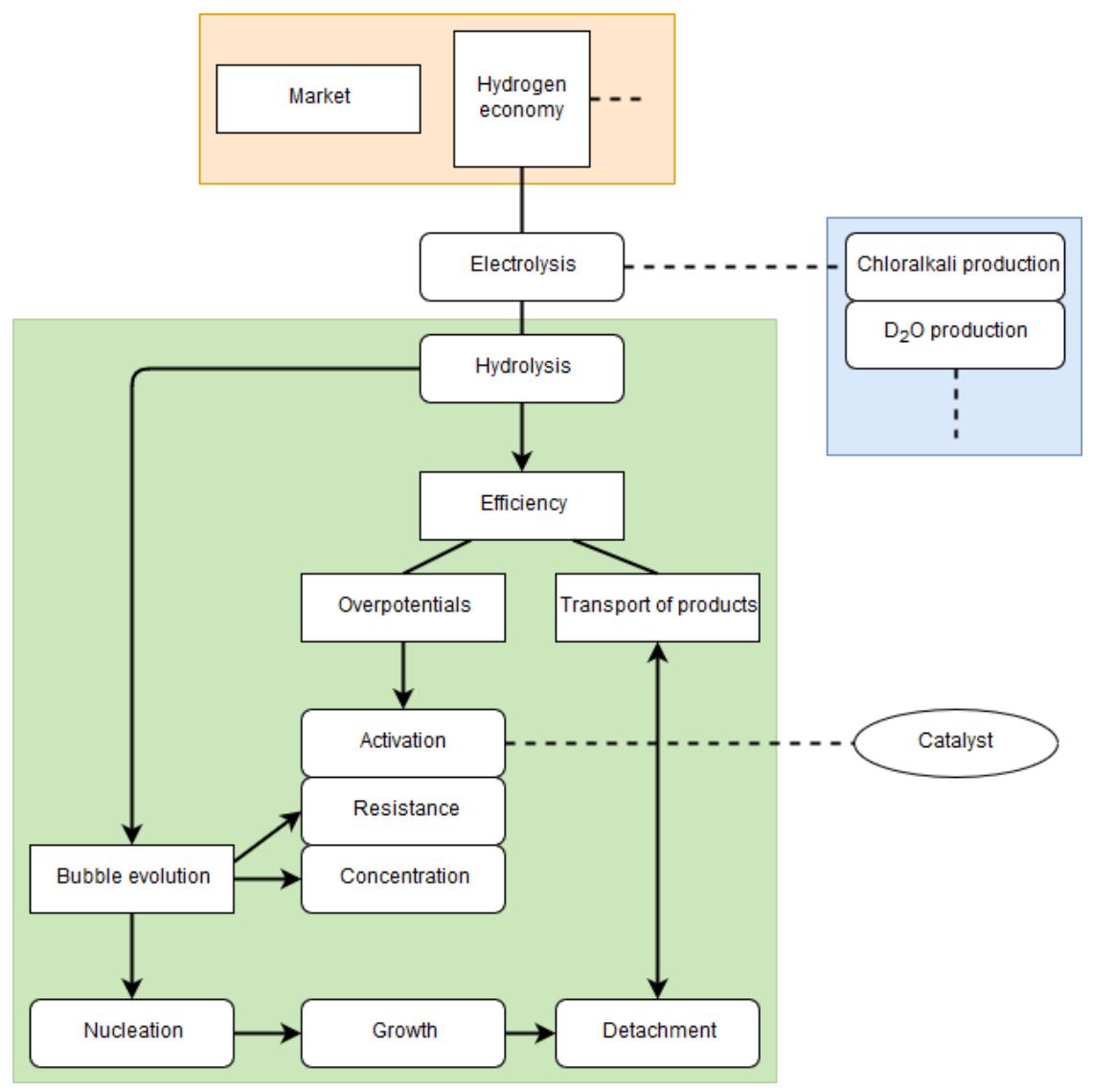

Figure 1.3: Schematic overview of the framework in which the research was carried out. The red rectangle shows part of the context this work does not concern itself with, the blue rectangle shows two examples where this work might be applicable, and the green rectangle shows the topics the research focuses on. The dashed lines indicate topics beyond the scope of this work. The arrows point towards items that are a subset of the items at the start of the arrows. The double arrow indicates that the two items influence each other. 


\subsection{Thesis structure}

Chapter 2 provides an overview of the existing literature on the phenomena associated with the overpotentials during bubble evolution. In Chapter 3 , microstructured silicon electrodes with artificial nucleation sites are used to study isolated successive bubble growth. The substrates are applied to evolve hydrogen bubbles formed with electrolysis, and $\mathrm{CO}_{2}$ bubbles that were formed with pressure regulated supersaturation of liquids. In Chapter 4, an extensive theoretical work on the bubble evolution on microstructured silicon electrodes covered in Chapter 3 is presented. Chapter 5 addresses the detachment of bubbles on superhydrophobic pits next to catalytic surfaces. In Chapter 6 the conclusions of this work are given and perspectives presented. Here membraneless electrolyzers and electric potential responses to bubble formation near large electrodes are covered. 


\section{2}

\section{Influence of bubbles on energy and mass transfer efficiencies of electrochemical systems}

Bubbles influence energy and mass transfer in electrochemical systems. Since hydrogen gas holds a pre-eminent position as a renewable energy source to partially replace the use of fossil fuels, hydrolysis is chosen as the model process for which bubbles and related phenomena are discussed. In this review, overpotential losses in electrolyzers and overpotential losses due to bubbles are covered. Also, the influence of surfactants on bubbles, the current fluctuations arising from bubbles, photoelectric losses due to bubbles, and techniques to remove bubbles are discussed. We close with a conclusion and an outlook where we elude opportunities for higher Faradaic efficiencies in electrolyzers. 


\subsection{Introduction}

The presence of bubbles on a surface during industrial processes or laboratory experiments, can be the expected result or the undesired consequence of a thermodynamic transition. For example, bubbles form an important component of many beverages [16]. Bubbles positively influence chemical processes, such as mixing [17], aeration of bioreactors [18], and reduce fouling by suspended particles [19]. However, bubbles can cause cell death in bioreactors [20], and reduce heat-transfer in convection boilers [21].

In electrochemical processes, bubbles are known to induce convection, enhancing mass transfer rates [22, 23], but also undesired blockage of electrode surfaces [24]. To provide insight in the current understanding of how bubbles affect reaction efficiencies, we provide here a literature overview on this topic. Our aim is to advance the knowledge on electrochemical processes to arrive at an improved design of electrochemical systems with enhanced operational efficiencies.

We have focused on electrolysis of water where hydrogen and oxygen bubbles are formed, but the discussed phenomena are relevant for other experimental conditions. For example in aluminum electrolysis bubbles can block electrodes [25], as well as shielding membranes and electrodes in chloralkali cells [26]. The electrochemical hydrolysis of water results in hydrogen and oxygen gas, directly observed as bubbles on the electrode surfaces once a given saturation level of gas has been reached.

Bubble evolution is defined as the nucleation, growth, and detachment from the surfaces where they were formed [27]. Bubble evolution phenomena have been studied in batch [28] and flow conditions [29], with the goals of increasing the fundamental understanding of bubble evolution and raising the Faradaic efficiency of hydrogen evolving electrolyzers.

The economic and environmental interest in the production of hydrogen gas with high Faradic efficiency in electrolyzers is discussed in the next section. This is followed by a discussion on the choice for hydrogen production by electrolysis in Section 2.2. In Section 2.3, we discuss the influence of the characteristic lengthscale on heat- and mass transfer, after which we describe overpotential losses in electrolyzers, followed by the influence of the specific bubble evolution stages on the Faradaic efficiency. We finalize with a conclusions and outlook section where we elude the opportunities given by higher Faradaic efficiencies in electrolyzers. 


\subsection{Hydrogen gas to replace fossil fuels}

The consumption of oil and gas from the limited reserves that are readily accessible on Earth is expected to lead to a demand larger than the supply over a period of several decades [1]. New sources of these fossil fuels have recently been discovered $[30,31]$ and advanced techniques are now used to extract from the more difficult to reach oil and gas sources. An example of such an advanced technique is the conversion of kerogen, a solid organic matter from sedimentary rocks, into synthetic oil and gas [32]. However, the sources are limited and an oil and gas shortage seems imminent in the long run [33].

With the expected oil and gas shortage and an unequal distribution of natural resources, a (partial) transition away from the use of fossil fuels is desired. This energy transition is often paired with tackling another global issue, the reduction of $\mathrm{CO}_{2}$ emission levels. To combat this two-fold problem, the use of renewable energy sources such as solar energy has been suggested [2].

The sunlight that reaches the Earth's surface in less than one hour exceeds the yearly energy consumption by mankind [2, 4]. Due to this abundance of solar energy, there is a large interest in the use of this renewable source to address the energy shortage challenge. The energy supply of solar light and the energy demand of end users varies in time, due to transition from day to night, seasonal changes, etc. [34], this intermittency problem needs to be addressed by ways of storing solar energy.

Hydrogen [35], methanol [36], and chemical conversion of energy such as in lithium ion or sodium/sulfur batteries [37] have been demonstrated to function as energy carriers to store and release energy when required. Hydrogen holds a pre-eminent position to partially replace the use of fossil fuels [35, 38]. Hydrogen is a light-weight dense energy carrier, and upon combustion it is free of harmful products [39]. Furthermore, the possibility to produce hydrogen in a decentralized manner with the use of photo-voltaics or photo-electrochemical systems, can be advantageous for isolated communities that lack electrical grid access [6]. Hydrogen can be stored in (pressurized) cylinders, cryogenic liquid storage, and material-based storage [40] such as in metal hydrides [41], which addresses the intermittent supply and demand issues. It should be noted that the use of metal hydrides provides several benefits, such as the lack of hydrogen leakage probability, which are outweighed by several downsides such as the total weight per kilogram hydrogen storage and some of the hydrides reacting violently with air [42]. In previous studies, the use of hydrogen gas to replace fossil fuels has been discussed in great detail. 
The interested reader is referred to other works on this topic for further information [2, 35, 38].

Large volumes of oil and natural gas are consumed $(1.7 \mathrm{Mb} / \mathrm{d}$ and 96 bcm in 2017, respectively [43]), meaning that large quantities of hydrogen gas are required if all of the fossil fuels need to be replaced. Because the volumetric energy density of hydrogen with $4 \mathrm{MJ} / 1$ at $\sim 700$ bar is low compared to diesel with a volumetric energy density of $25 \mathrm{MJ} / 1$ [44]. Large storage and distribution capacity is required before hydrogen gas can truly become an attractive alternative. Solar-driven water-splitting has the potential for large-scale implementation, for both photo-electrochemical as well as photovoltaic systems [45]. The most relevant aspects influencing the energy efficiency of solar-driven water-splitting systems are therefore discussed in detail in the next sections.

\subsection{Electrolysis at the mesoscale}

At the mesoscale (the length scale between microscale and macroscale where individual atom behaviour can be excluded when addressing material properties or phenomena), heat- and mass transfer rates are increased due to shorter lengths scales [46] and increased surface to volume ratios [47].

Electrolyzers operate more efficient at elevated temperatures due to the lowered power requirement by enhanced thermodynamics and kinetics of the reactions involved [48] and increased electrolyte conductivity [49]. Diffusion of species often is the limiting mass transport mechanism, due to its relative slow nature. For example, in water dissolved hydrogen and oxygen gases have diffusion coefficients of $4.5 \times 10^{-9} \mathrm{~m}^{2} / \mathrm{s}$ and $2.1 \times 10^{-9} \mathrm{~m}^{2} / \mathrm{s}$, respectively [50]. Enhanced mass transfer can be achieved by electrolyzers with mesoscale dimensions, which can translate to a micrometer separation distance between cathode and anode and results in short species residence times. The electrode spacing is further related to the electrical resistance of the electrolyte volume between the electrodes [47, 51]. Small separations result in a low electrolyte resistance, and large separations result in losses from the ionic transport [46]. The void fraction, a measure of the void created by bubbles in the electrolyte, is related to the spacing, height, inclination of electrodes, current density, and parameters related to cell design such as; material choice, electrolyte, and cell operating conditions $[47,51]$. High void fractures result in increased overpotentials, which reduce the electrolysis efficiency as will be discussed in Section 2.4.3. 


\subsection{Overpotential losses}

Electrolysis efficiency is determined by the overpotential losses which can be categorized in activation, resistance, and concentration overpotentials. We discuss in the next subsections the overpotential changes caused by bubbles as a special case of the concentration and resistance overpotentials. Figure 2.1 illustrates the overpotential losses in electrolyzers by setting out the potential of the electrolyzer cell (cell voltage) as function of the current density.

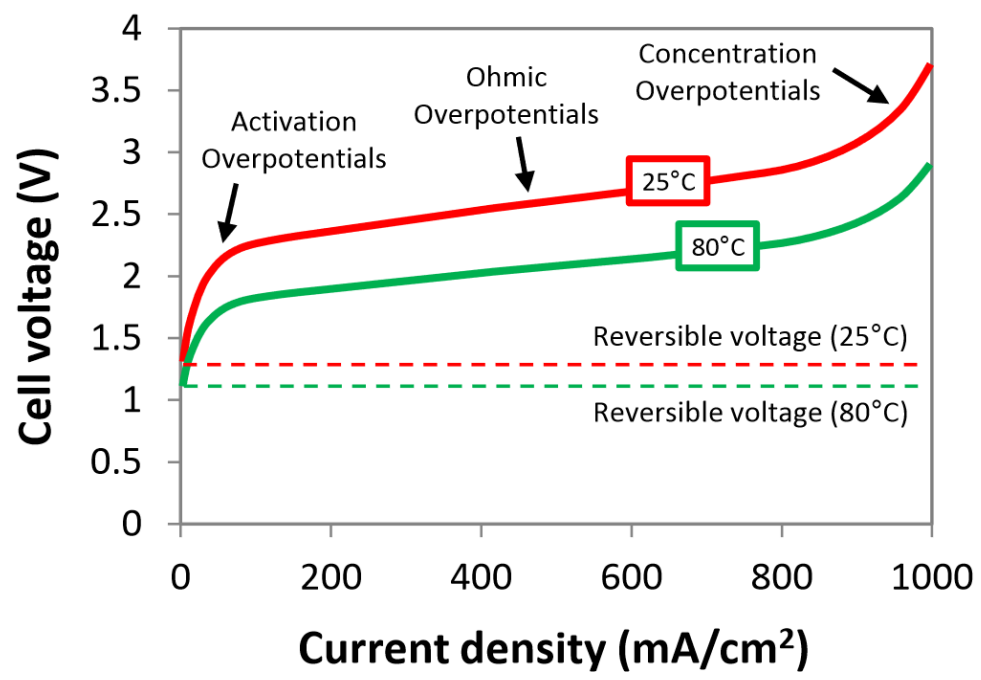

Figure 2.1: The electrolyzer cell voltage is shown as function of the current density for electrolyzer cell operation at two temperatures (operation at $25^{\circ} \mathrm{C}$ and $80^{\circ} \mathrm{C}$ shown by the red and green curves, respectively). The components of the total overpotential are indicated on the polarization curve. The dashed lines show the reversible voltages at $25^{\circ} \mathrm{C}$ (top line) and $80^{\circ} \mathrm{C}$ (bottom line). Reprinted with permission from (Amores, E., Rodríguez, J., Oviedo, J., et al. (2017). Development of an operation strategy for hydrogen production using solar PV energy based on fluid dynamic aspects. Open Engineering, 7(1), pp. 141-152. doi:10.1515/eng-2017-0020). (C) 2017 Ernesto Amores et al.. This work is licensed under the Creative Commons Attribution-NonCommercial-NoDerivatives 3.0 License. BY-NC-ND 3.0 


\subsubsection{Activation overpotential}

The activation overpotential is the potential loss from the kinetics of electrochemical reactions at the electrodes. The Butler-Volmer equation, shown in Equation 2.1, expresses the operational current density as a function of exchange current density and the activation overpotential of both electrodes.

$$
j=j_{0}\left[e^{\left(\alpha_{a} z F \eta / R T\right)}-e^{\left(-\alpha_{c} z F \eta / R T\right)}\right]
$$

with $j_{0}$ the exchange current density in $\mathrm{A} / \mathrm{m}^{2}, \alpha_{a}$ and $\alpha_{c}$ the anodic and cathodic charge transfer coefficients respectively, $z$ the electron valency, $F \approx 96485 \mathrm{C} / \mathrm{mol}$ the Faraday constant, $\eta$ the activation overpotential in $\mathrm{V}, R \approx 8.314 \mathrm{~J} / \mathrm{K} \cdot \mathrm{mol}$ the universal gas constant, and $T$ the absolute temperature in K. During electrolysis of liquids, conditions such as, temperature, electrolyte composition, $\mathrm{pH}$, and material choices, determine the exchange current density [52], since the dissociation potential of a liquid is influenced by the catalytic activity of the electrodes [53]. For example, $\mathrm{IrO}_{2}$ film electrodes for the oxygen evolution reaction are typically used in acidic environments [54], and Ni-Fe film electrodes for the oxygen evolution reaction were found to function with higher activity in alkaline solutions [55]. Exchange current densities, as well as the Tafel slopes $\left(\eta / \ln \left(\mathrm{j} / \mathrm{j}_{0}\right)\right)$ of various materials often employed in electrolysis have been tabulated [56]. It should be taken into account that some of the listed materials can be covered by an oxide film. The presence of an oxide layer on the reacting surface may reduce the reaction rate, e.g. because of a lower adsorption energy [57]. Another relevant review in the context of solar driven water splitting [58] provides a thorough overview of photo-anode and photo-cathode materials. An extensive list of catalytic materials for photo driven water splitting was given in another review [59], including their activity for $\mathrm{H}_{2}$ and $\mathrm{O}_{2}$.

\subsubsection{Resistance overpotential}

Resistance overpotentials in electrolyzers arise due to the presence of junctions, such as those of membranes used to prevent the crossover of species from one electrode to the other. Gaseous species such as oxygen and hydrogen are often kept separated to prevent the formation of explosive mixtures [60]. This can be done by using proton exchange membranes that allow for the passage of protons but with pores too small for gas species to cross. Materials that are used for membranes include polymers such as Zirfon [61], Nafion, Flemion, and Aciplex [62]. Among the limiting conditions in the use of membranes are high temperatures (for 
polymer membranes), chemical stability [63], mechanical strength, proton conductivity, degree of crossover, clogging of membranes, and costs. A recent review [64] elucidated that the presence of a membrane can indeed increase the cell resistance by the electrical resistivity of the membranes. Depending on the specific cell design, membranes can help minimize the interelectrode spacing. This in turn contributes to reducing the electrolyte resistance. The interested reader is referred to recent literature $[53,64]$ on the current developments on this topic.

Several membraneless alternatives have been employed in electrolyzers where the formed products have successfully been separated. A membraneless electrolyzer which operates with electrodes placed under angles has been reported [65]. The orientation of the electrodes and buoyancy of detaching bubbles was used to separate the products, hydrogen crossover rates as low as $1 \%$ have been reported. A membraneless electrolyzer using electrolyte flow to separate the products has also been demonstrated [66]. A microfluidic channel with T-junction (one inlet and two outlets) was created over two electrodes, the flow-induced velocity profile ensured that formed gas was separated at the two outlets. At the channel walls, a slower flow occurs than in the center of the channel, thus effectively the products were pushed to the sides by a net intertial lift force. Crossover percentages below 5\% were achieved with flow rates of $10 \mathrm{ml} / \mathrm{h}$ and above. Impedance spectroscopy was used to measure device resistances below $110 \Omega$ for various electrolytes and higher conductivity values compared to Nafion with the same electrolyte.

\subsubsection{Overpotential changes due to bubbles}

Bubbles can cause overpotential changes when attached to electrodes employed in electrochemical reactions. The void fraction arising as bubbles form affects several parameters for example net cell resistance is increased [67], electrolyte conductivity is lowered [68], and the total overpotential is increased typically. Decreasing total overpotential values have been reported in studies $[69,70]$ by a lowered concentration overpotential component under specific conditions.

The effects of the void fraction on the overpotential have been investigated extensively. The influence of interelectrode spacing on the overpotential showed that large increases in overpotentials arise when large void fractions are present between closely spaced electrodes, however interelectrode spacing above $10 \mathrm{~mm}$ resulted in little resistivity changes because bubbles had a negligible contribution to the much larger total resistance from the electrolyte between the electrodes [71]. Overpotentials were further investigated by the influence of electrode 
spacing, current density, membrane, temperature, inclination angle, and electrode wettability on the void fraction [51]. An increased void fraction and decreased electrolysis efficiency was found upon decreasing the spacing between the electrodes.

Void fraction, Ohmic resistance, and the current density have been measured in experiments where the electrolyte volumetric flow rate was varied as well as the spacing between the electrodes [72]. Both a decreased flow rate and electrode spacing resulted in a larger current density. More recently, it was found that the void fraction solely as function of current density can be used for straightforward predictions using a semi-empirical equation:

$$
\frac{I / A}{I_{S U} / A}=3.08 \Theta^{1.5}(1-\Theta)^{0.5}
$$

with $I$ the current, $A$ the electrode area, $I_{S U}$ the maximum current, and $\Theta$ the fractional bubble coverage of the electrode surface. Parameters such as pressure, temperature, and residence time of bubbles need to be taken into account for more precise predictions [24].

The overpotential increase has been modeled [73] taking into account a stagnant electrolyte layer at the electrodes and void fraction. The theoretical model was compared with experimental findings [71], the measured resistivity was found larger than the model predictions. The larger resistivity was attributed to possible variations in the liquid layer thickness where the bubbles resided, with respect to flow conditions that could arise in the experimental study. The overpotential caused by bubbles, and electric current distributions around bubbles was investigated [74]. The study showed how bubbles increase electrolyte resistance by deforming the current path. It was concluded that the current density reaches small values near the contact line of a bubble in contact with an electrode surface.

Due to strong coupling between various overpotential effects caused by bubbles, it proved difficult to study one specific contributor to the total overpotential [69]. The contribution of the concentration and Ohmic overpotentials to the total overpotential was investigated [69]. The study focused on the influence of evolving bubbles attached to gas-generating electrodes, taking into account the potential drop and current distribution around the bubbles. The authors considered two effects exerted by the gas bubbles in detail. Firstly, a bubble on a gas-evolving electrode has a certain footprint related to the wettability of the electrode, and the surface is blocked for further reaction where the bubble is in contact with the electrode. Secondly, evolving bubbles take in dissolved gases present in the electrolyte, reducing local gas concentrations surrounding the bubble which changes the concentration overpotential [70]. 
A theoretical model predicting voltage effects caused by multiple bubbles, has been proposed [69]. In the model it was assumed that transport phenomena are pseudo-static with respect to the bubble growth, with spherical bubbles all having the same radii. The multi-bubble problem was approached by using an equal area cylinder approximation around each bubble. The packing density, indicating the ratio of the area covered by the cylinders over the total area, was found to influence the current distribution. Furthermore, the maximum current density increased with the increased packing density. It was reasoned that the current goes through the unmasked electrode area, and the reduced surface area resulted in a larger maximum current density. Moreover, an increased resistance was found with larger packing densities, in agreement with void fraction studies $[51,71,72,75,76]$. The theoretical prediction of a differential resistance increment, as a function of the packing density, was set out against experimental measurements [77] and showed good agreement. The model showed that, near the bubble contact line, the current density is highest when taking into account the locally decreased gas concentration and concentration overpotential.

The model predicted that the differential potential increase could be negative under certain conditions. The dimensionless differential potential $\Delta V^{*}=\ln \left(\frac{1}{1-\sigma_{s}}\right)$ with $\sigma_{s}=\sqrt{3} \pi \sin ^{2} \Theta / 6 \mathrm{~s}^{2}$, where $\Theta$ is the contact angle of the bubble, and s the interbubble spacing, is shown as function of the dimensionless current density $\delta$ in Figure 2.2. Where

$$
\delta=\left(\alpha_{a}+\alpha_{c}\right) \frac{i_{A V G} a}{R T \kappa / F},
$$

with $\alpha$ the charge transfer coefficient (the fraction of the interfacial potential between electrode and electrolyte that assists the reduction of the free energy barrier for the electrochemical reaction), subscripts $a$ and $c$ specify the anode and cathode respectively, $i_{A V G}$ is the average current density in $\mathrm{A} / \mathrm{cm}^{2}, a$ the radius of the bubbles, $R \approx 8.314 \mathrm{~J} / \mathrm{K}$ mol the gas constant, $T$ the temperature, Faraday's constant $F \approx 96485 \mathrm{C} / \mathrm{mol}$, and $\kappa$ the conductivity of the liquid phase in $1 / \Omega \cdot \mathrm{cm}$. The various curves in Figure 2.2 indicate the modeled result from various dimensionless exchange current densities, their magnitudes are indicated next to the corresponding curves. The graph shows that with slow electrode kinetics a large voltage drop exists as function of increased current density. Most interestingly, with fast kinetics a negative dimensionless voltage increment arises at low dimensionless current density. At a high exchange current density and at low current density, the bubbles attached to the electrode deplete their local surrounding liquid phase from dissolved gas. The gas depletion in the liquid causes a local net depolarization of the electrode, resulting in 
a lowered overpotential. At high current densities with fast kinetics, this depletion effect is diminished by the large influx of gas, resulting in a larger concentration gradient which counteracts the depletion effect.

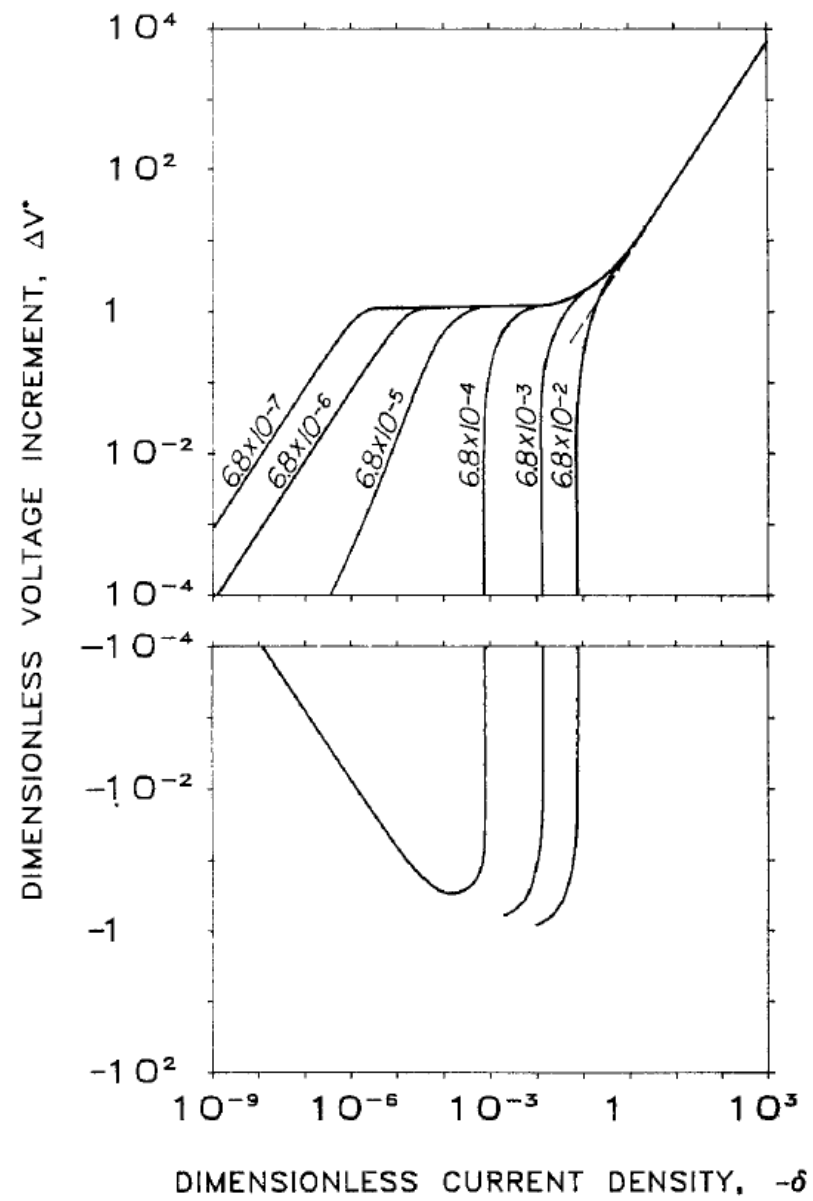

Figure 2.2: Dimensionless voltage increment $\left(\Delta V^{*}\right)$ due to bubbles attached to electrodes as function of dimensionless current density $(-\delta)$ for various exchange-current densities. The top figure shows the positive and the bottom figure shows the negative voltage increments due to reduced concentration overpotentials. Republished with permission of Electrochemical Society, Inc, from The Influence of Attached Bubbles on Potential Drop and Current Distribution at Gas-Evolving Electrodes, J. Dukovic and C.W. Tobias, Journal of the Electrochemical Society, volume 143, issue 2 1987; permission conveyed through Copyright Clearance Center, Inc. 
In an experimental study [70] the concentration overpotential was found by spectral analysis of the measured current. The prediction of the lowered overpotential in the model of Dukovic et al. (1987) [69] was verified using ferricyanide in an alkaline medium to form hydrogen gas on a circular platinum electrode with $5 \mathrm{~mm}$ radius. With a high exchange current at low cathodic current densities of $-1.8 \mathrm{~mA} / \mathrm{cm}^{2}$, resistance drops $(\sim 20 \mathrm{~m} \Omega)$ were measured upon an increased area of the electrode being exposed to the electrolyte.

\subsubsection{Electrolytic bubble evolution}

The upcoming sections elucidate the nucleation, growth, and detachment stages (shown in Figure 2.3) followed by a section on the influences of bubble evolution on electrolysis measurements.

A) Nucleation

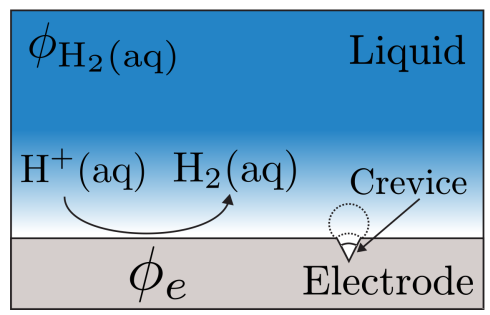

B) Growth

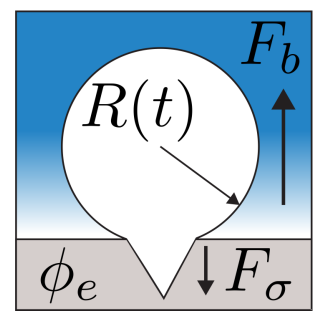

C) Detachment

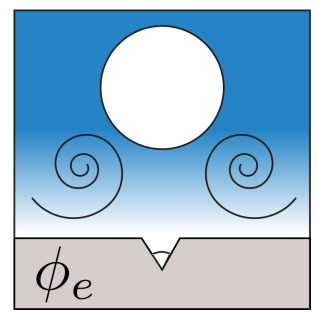

Figure 2.3: The various stages of bubble evolution. From left to right, the nucleation, growth, and detachment of bubbles on a hydrogen gas evolving electrode surface. Nucleation occurs typically on cracks and crevices in the electrode surface, after which the bubble grows by taking in gas from the dissolved gas boundary layer. The bubble detaches (for upward facing electrodes) when the buoyancy force overcomes the interfacial tension force. The departing bubble induces convection in the liquid indicated by the spirals. Adapted from Ref. [27] with permission from The Royal Society of Chemistry.

\section{Nucleation of electrolytic bubbles}

During electrolysis, the bubble nucleation process is driven by a chemical potential caused by an amount of dissolved gas molecules in the liquid phase [78]. Nucleation occurs when the chemical potential is large enough such that dissolved gas molecules can overcome the thermodynamic energy barrier associated with forming a gas bubble nucleus [78, 79, 80]. The nucleation of bubbles occurs typically at the electrode surface since heterogeneous nucleation has a lower thermodynamic barrier than 
homogeneous nucleation in the bulk [81, 82, 13, 83, 84]. A study of the nucleation of hydrogen bubbles near a mercury pool electrode surface [85], concluded that gas bubbles underwent homogeneous nucleation in the electric double layer next to the electrode surface. This was attributed to an intense electric field $\left(-2.3 \times 10^{8} \mathrm{~V} / \mathrm{m}\right)$ in the electric double layer, originating from the polarization of the liquid molecules lowering the local surface tension, which reduced the nucleation energy barrier.

The homogeneous nucleation of bubbles has also been investigated at a gold electrode (25 $\mathrm{nm}$ diameter) using in situ transmission electron microscopy [84]. Using a high spatial and temporal resolution, it was demonstrated that the nucleation occurred several nanometers away from the electrode surface before the formed nucleus came in contact with the electrode. Figure 2.4 shows the bubble situated next to the gold electrode with a $7 \mathrm{~nm}$ space between electrode and bubble. The homogeneous nucleation, attributed to the electrode wettability, was followed after $2 \mathrm{~s}$ by dewetting the electrode in a thermodynamic irreversible interaction.

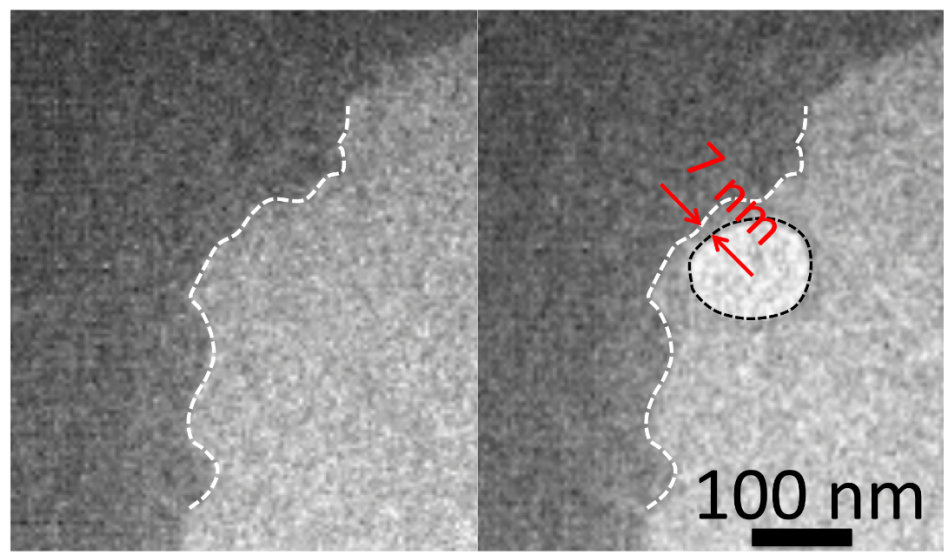

Figure 2.4: In situ transmission electron microscopy images of the nucleation of a hydrogen bubble next to a gold electrode, the dashed line shows the outline of the electrode. The left image shows the electrode before nucleation, the right image shows the bubble adjacent to the electrode. The time step between the two images is $340 \mathrm{~ms}$ and the distance between bubble and electrode is $7 \mathrm{~nm}$. Republished with permission of ROYAL SOCIETY OF CHEMISTRY, from In situ observation of electrolytic H2 evolution adjacent to gold cathodes, Y. Liu and S.J. Dillon, Chemical communications, volume 50, issue 14 2014; permission conveyed through Copyright Clearance Center, Inc. 
Both homogeneous as well as heterogeneous nucleation processes are described for single gas systems in classical nucleation theory, which combines thermodynamics and kinetics [13]. The thermodynamics determine the activation barrier, based on the cohesive force of the liquid, whilst assuming a critical radius for the bubble nucleus which is large enough that bulk thermodynamic properties apply [86]. Since electrolysis of water is a process in which multiple gaseous components are formed, the nucleation theory takes these components into account [87]. However, theoretical nucleation rates and required supersaturation levels for nucleation during electrolysis are often higher than their experimental counterparts $[88,89]$. Several examples of this mismatch are given in a review [13] and were attributed to the fact that often the surface tension was not accounted for, which incorrectly lead to nucleation predictions independent of gas type [88].

It was proposed that the origin for the theoretical inaccuracy in predicting the supersaturation levels originated from the bubble shape, a spherical volume that is taken for the initial phase change [90]. The likeliness for all the molecules to undergo the phase transition with the increased pressure inside the bubble was doubted since this would cause a steep increase in molecule density. An alternative approach was proposed in which a blob of unknown shape would be the initial volume of the phase change which could account for the mismatch in supersaturation levels. Here the Helmholtz energy to form the blob was found lower than a bubble nucleus calculated with classical nucleation theory, since less work was required to form the gas liquid interface.

Isolated bubbles have been formed in electrolysis with nano- and micro-sized electrodes [79, 91, 92, 93, 94, 23]. Isolated bubbles are by definition not under the influence of interactions with other bubbles such as Ostwald ripening, convection upon detachment of bubbles in close vicinity, and coalescence phenomena. Studying isolated electrolytic bubbles resulted in improved understanding of the dissolved gas boundary layer on nucleation [79], bubble growth [28], and the mass transfer in spatial confinement of the electrolyte and bubble [94].

The nucleation of hydrogen bubbles in spatial confinement was studied using glass pores of $10 \mathrm{~nm}-100 \mathrm{~nm}$ in radii, which exposed an underlying platinum disk [94]. As a result of the design of the pores, the diffusion of $\mathrm{H}_{2}$ was restricted to the pore, which allowed for calculation of a $0.22 \mathrm{M} \mathrm{H}_{2}$ supersaturation concentration at time of the nucleation and the observation of an anodic peak during a reverse voltammetric scan related to the oxidation of dissolved hydrogen gas.

Inverted pyramidal structures etched into silicon with a width of $40 \mu \mathrm{m}$ at the top, covered with titanium and gold layers, formed electrodes used 
for electrolysis [79]. Due to the shape of the cavity it was reasoned that the dissolved gas concentration gradients, formed at the electrode surface, would overlap in the apex of the cavity, resulting in a highly oversaturated area during electrolysis and to bubble nucleation inside the cavity.

Other ways to structurally define the onset location for bubble nucleation are the formation of bubbles on needle electrodes [95]. The influence of the frequency of an applied AC voltage during electrolysis on a needle-shaped electrode was investigated. A frequency range, depended on the apex of the needle, in which the nucleation of bubbles occurred at a single nucleation site on the electrode was found. This was attributed to the convergent electric field at the tip of the electrode controlling the charge transfer rate. The onset location for nucleation was also defined used pits and scratches deliberately made on electrodes surfaces [96], and changes in hydrophobicity [97, 98]. Recently, bubble nucleation and the influence of the local dissolved gas boundary layer on the growth of bubbles on superhydrophobic pits has been studied [28]. Here the superhydrophobic pits were micromachined in silicon electrodes that were employed as cathodes in water splitting reactions.

\section{Electrolytic bubble growth}

Different growth regimes occur during bubble evolution in electrolysis. The initial stage of growth is governed by inertia [99], and lasts around 10 ms [92]. This stage of growth is characterized by a growth rate that can be described by: $R_{b}=\tilde{b} t[92,100]$, with $\mathrm{R}_{b}$ the radius of the bubble, the dimensionless growth coefficient $\tilde{b}=b / \sqrt{D}$ with $b$ a growth coefficient, $D$ the gas diffusion coefficient, and $t$ the time. The second stage is governed by mass transfer. When the diffusion of gases is the governing mass transfer mechanism, the growth of bubbles can be described by: $R_{b}=\tilde{b} t^{1 / 2}$. The diffusion-controlled bubble growth has been extensively covered in literature. The works of Epstein and Plesset [14] show the approximate solutions for bubble growth of a free bubble in undersaturated and supersatured liquid-gas solutions. Later Scriven [101] took into account the advection term in the diffusion equation, providing an exact solution for the diffusion controlled bubble growth. Diffusion controlled bubble growth during electrolysis occurs once the liquid surrounding the bubble saturates over time such that diffusion of dissolved gas occurs from the bulk electrolyte towards bubbles located on the electrode. Thus, diffusion governed growth can occur on large electrodes were gas formation partially happens far away from the bubble. Diffusive growth can occur in boiling [102] and pressure driven supersaturation [103] as well by the saturation of the bulk liquid. When the reaction rate limits bubbles 
growth, the growth can be described by: $R_{b}=\tilde{b} t^{1 / 3}$ where the exponent originates from the volumetric gas addition [92, 23, 96, 104, 105]. Reaction governed growth occurs once the distance between reacting surface and bubble is short enough for diffusion effects to become negligible. Typical systems in which reaction governed mass transfer can be observed use nano- and microelectrodes. Although most electrolysis systems follow the aforementioned types of growth, deviation from this can occur. A moving gas-evolving source was studied [106], where a bubble growth following $R_{b}=b t^{\sim 1 / 4}$ was measured. Deviations from the diffusion governed growth were also observed during bubble growth in an underdeveloped dissolved gas boundary layer [28].

\section{Bubble detachment}

Bubble detachment is the process of a bubble unpinning from a surface. This process has been researched extensively particularly in theoretical studies [89, 15, 107] and during electrolysis in experimental studies [27, 92, 93, 97, 108].

Bubble evolution during boiling and electrolysis of liquids is analogous to the transport of substances [109]. It was shown that when the current density of the gas evolving electrode was kept low enough to prevent wettability changes by the electrode potential, calculations using empirical equations concerning bubble detachment radii for both boiling and electrolysis were in agreement.

Studies on bubble detachment that do not make use of boiling, nor electrolysis, typically focus on directly injecting gasses into liquids $[15,110$, $111,112]$ or pressure driven oversaturation $[103,113]$. Single bubbles can be formed with the injection of gas in a liquid. Control can be exerted over the type of gas and liquid conditions such as adiabaticity [110]. Similarly, control over liquid conditions such as pressure, temperature, and the type of liquid and gas in pressure driven supersaturation is available. Pressure driven $\mathrm{CO}_{2}$ oversaturation has previously been compared with electrolysis where liquid conditions, such as gas concentration, are dynamic [27, 28]. The bubble detachment occurred at comparable radii from the same micromachined artificial nucleation sites with either technique. An attenuation in the detachment radii of successive bubbles in the pressure driven system occurred due to density driven convection [113].

Early work on bubble detachment, covered the force balance between buoyancy force and surface tension in which the necking of the bubble was empirically accounted for [114]. Bubble detachment was further covered in pool boiling were the detachment size of bubbles was experimentally and theoretically investigated [115]. Later, the detachment 
of electrolytic bubbles from platinum microelectrodes has been thoroughly investigated [92]. Microelectrodes with various radii were employed to study the effect of $\mathrm{pH}$ on the net charge induced on the bubble interface, the influence of electrostatic interactions on detachment radii of bubbles, screening effects, and the influence of surfactants. The influences of electrostatic interactions are further highlighted in Section 2.4.4.

Coalescence often precedes the detachment of bubbles because the bubble is mechanically forced to detach by the expanding boundaries of the bubble [89]. Also liquid flow [17, 116] tends to promote bubble detachment, where wakes or convection caused by bubbles promote the detachment of neighboring bubbles [17, 117]. Bubbles experience additional forces due to movement of the liquid, resulting in the onset of detachment at smaller radii. A force balance further showed that bubbles originating from an orifice in a wall that experiences gravity influences and cross-flow, detach at smaller radii with increased flow rates due to the induced shear stress [118]. In Section 2.4.5, methods to induce early bubble detachment and the response caused to the total overpotentials are discussed further.

\section{Electrical charge and surface tension influence on the detachment of bubbles}

Measurements in surfactant free electrolytes showed a decrease in hydrogen bubble detachment diameters with increased $\mathrm{pH}$ levels [92]. The decreased detachment diameters were the result of repulsive electrostatic interaction between the charge on bubbles, induced by $\mathrm{pH}$ via the accumulation of protons and hydroxide ions on the bubble interface [119], and the electrode. Below $\mathrm{pH} 3$, the bubble had a net positive charge, at $\mathrm{pH} 3$ the bubble had no net charge, and at values higher than $\mathrm{pH} 3$ the bubbles were negatively charged [92]. Similarly, oxygen bubbles evolving on the anode detached with increased radius at higher $\mathrm{pH}$ levels due to the $\mathrm{pH}$ induced bubble charge

The effects of Sodium dodecyl sulfate (SDS) and Dodecyltrimethylammonium bromide (DTAB) on electrolytic bubbles were investigated. Addition of SDS resulted in both hydrogen bubbles (with $\mathrm{pH}$ below 3) and oxygen bubbles (with $\mathrm{pH}$ above 3) detaching at smaller radii, due to the Coulombic forces being reduced by the adsorption of negatively charged SDS on the bubble and anode, respectively. Addition of the positively charged DTAB had the same effect, hydrogen bubbles (with $\mathrm{pH}$ below 3) and oxygen bubbles (with $\mathrm{pH}$ above 3) detaching at smaller radii. However, the effects where now caused by adsorption of $\mathrm{DTAB}$ on the cathode for hydrogen bubble and adsorption on the bubble 
interface for oxygen bubbles due to the charge of DTAB being opposite in sign with respect to the charge of SDS.

Dissolved gases can reduce the surface tension of the liquid phase [120, 121]. A change in surface tension could result in soluto-capillary flow [122], also known as Marangoni flow. Although Marangoni flow has been hinted at to play an important role during electrolysis [123], little research has been published on the topic. In a recent publication [124], an experimental study employing particle tracking velocimetry measured the Marangoni flow around hydrogen bubbles generated via electrolysis. Marangoni flow velocity was increased with elevated current density levels. Two possible causes for this were given: an enhanced soluto-capillary flow originating from the larger gas flux with increased current density, or increased Joule heating which would result in thermo-capillary flow. However, no discrimination between the two mechanisms could be made.

\section{Electric current fluctuation by evolving bubbles}

The presence of bubbles on electrodes can exert influence on the overpotential measured as discussed in Section 2.4.3. The bubble evolution stages during electrolysis have been correlated to changes in currents or potentials measured. Nano- or micro-electrodes are employed to form a small number of bubbles on the electrode surface such that the screening area, and therefore fluctuation in potential, is relatively large. Other techniques make use of noise spectra analysis, typically used in systems where larger number of bubbles evolved.

The overpotential resulting from evolving bubbles on platinum microelectrodes with diameters of $125 \mu \mathrm{m}$ was investigated [93]. The optical recording of the evolution of bubbles on the platinum microelectrode was correlated with the measured overpotential. Figure 2.5a shows at the top the measured overpotential and at the bottom the measured contact angle as function of time. The numbers in the graph correspond to the numbered images in Figure 2.5b, depicting the various stages in the bubble evolution. It is shown that the bubble in contact with the electrode has the same order of magnitude in radius as the electrode radius. During the bubble evolution, three stages were found. The first stage is characterized by a decreasing contact angle whilst the overpotential increased. The second stage has a small contact angle and decreases slightly while the overpotential becomes a little less negative. The third stage shows the necking process in which the bubble neck decreases till the interface comes in contact with itself and the bubble detached. No explanation was given for the measured overpotential and the decreased values in the second stage. 

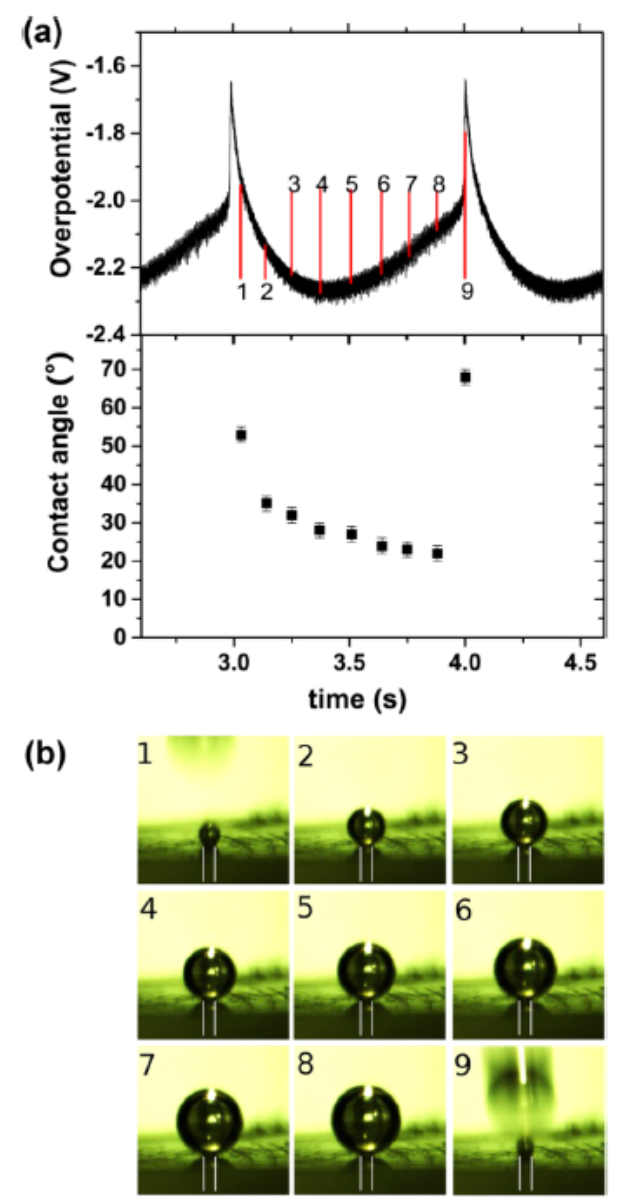

$800 \mu \mathrm{m}$

Figure 2.5: Bubble evolution on a platinum microelectrode with a diameter of $125 \mu \mathrm{m}$. A) The top figure show the overpotential as function of time during the evolution of a single bubble. The bottom figure shows the contact angle during the bubble evolution as function of time. B) Images taken during the bubble evolution, where the numbers in the images indicate their time in the overpotential measurement shown in (A). The electrode is indicated by the white lines. Reprinted with permission from (Bubble Formation at a Gas-Evolving Microelectrode, Damaris Fernández, Paco Maurer, Milena Martine, J. M. D. Coey, and Matthias E. Möbius, Langmuir 201430 (43), 13065-13074, DOI: 10.1021/la500234r). Copyright (2019) American Chemical Society. 
Simultaneous acquisition of the current, during a water splitting reaction with a $-1.5 \mathrm{~V}$ constant potential, and bubble evolution on a platinum microelectrode with a diameter of $100 \mu \mathrm{m}$ has been reported [23]. In Figure 2.6 a graph is shown with the measured current as function of time. The letters inside the graph indicate which stage of bubble evolution, shown in the image sequence below, corresponds to the current measured. It can be seen that in image A the bubble is detached from the platinum electrode where several small bubbles have nucleated. The current profile shows that the current is about $-1.55 \mathrm{~mA}$ and with increased bubble volume shown in images B-D the current decreases with about $0.2 \mathrm{~mA}$. After 1.65 $s$ the current increases whilst the bubble continues to grow (images E \& F), the authors attributed this to the bubble moving to the edge of the electrode effectively screening a smaller part of the electrode with the bubble.
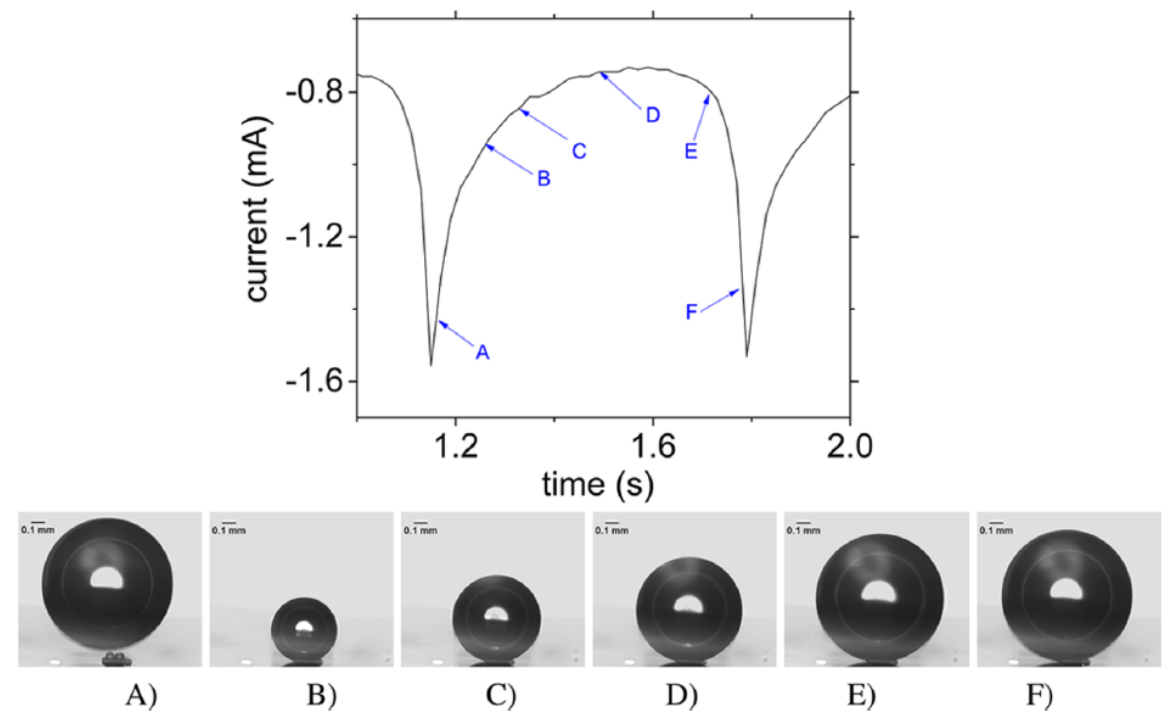

Figure 2.6: The top figure shows the measured current as function of time at a constant potential of $-1.5 \mathrm{~V}$ during the evolution of an isolated bubble on an upward-facing microelectrode. The numbers indicated near the curve show where the images in the bottom figure correspond in time. The bottom images show the bubble evolution as function of time. Reprinted with permission from (Dynamics of Single Hydrogen Bubbles at a Platinum Microelectrode, Xuegeng Yang, Franziska Karnbach, Margitta Uhlemann, Stefan Odenbach, and Kerstin Eckert, Langmuir 2015 31 (29), 8184-8193, DOI: 10.1021/acs.langmuir.5b01825). Copyright (2019) American Chemical Society. 


\section{Photoelectric losses caused by bubbles}

In electrolysis driven by illumination, losses in the photo-driven processes can occur by the presence of bubbles [125, 126]. Incident light can be scattered resulting in light that has a reduced intensity reaching the surface of the electrode underneath the bubbles. Due to the shapes of bubbles and the change in refractive index between the gas and liquid phase, incident light follows different pathways depending on the position on the bubble with respect to the light source. Snell's law allows for the calculation of the pathways through the bubble. This method has been applied in studies of photocurrent changes caused by the presence of bubbles in electrolysis.

The effects of illumination on single isolated bubbles during electrolysis on a $\mathrm{TiO}_{2}$ nanorod array have been studied [125]. On this photoanode, oxygen bubbles were formed and an initial increase in local photocurrent was measured due to the incident light on growing bubbles being scattered towards the triple contact line. Upon bubble growth, the area absorbing the scattered light became larger which led to an attenuation of the rate with which the intensity increased. The influence of bubbles on the photocurrent have been investigated in incident light measurements on photoelectrodes [126]. Several pathways for the incident light through the bubble have been identified using Snell's law. The theoretical work was supported by scanning photocurrent microscopy measurements, which showed that for large bubbles (diameter $\sim 1000 \mu \mathrm{m}$ ) photocurrent losses of $23 \%$ were measured. For small bubbles (diameter $\sim 150 \mu \mathrm{m}$ ) $2 \%$ photocurrent losses were recorded.

\subsubsection{Bubble removal and the electric response in electrolyzers}

Several techniques are available for the removal of bubbles. Here we discuss the influences of flow and ultrasound on the efficiency of electrolyzers and the detachment of bubbles.

Flow has been induced to ensure the early detachment of bubbles. Recently a study made use of pressure driven oversaturation to saturate liquid water with air [29]. Cavities etched in microchannels were used as artificial nucleation sites for bubble evolution. It was observed that with increasing liquid flow rates, smaller gas bubbles detached.

Bubble growth and detachment under shear flow near a wall has been studied [112]. A force balance model which predicted the radii of departing bubbles as function of gas flow rate and flow shear rate was provided. With increased flow shear rate the bubble radii measured decreased. Another study [108] made use of a micro-electrolyzer consisting out of a microfluidic channel fitted with electrodes for water splitting. As the flow velocity was increased, the bubble departure diameter decreased. It was observed that 
this was due to the bubbles being teared from the electrodes. Furthermore, their study found that with increased applied voltage the bubbles detached at smaller radii which was attributed to the influence of convection induced by detaching bubbles and electrostatic repulsions between bubble and electrode.

\section{Electrohydrodynamics and ultrasound}

Magnetic fields have been imposed in electrolyzers to induce a Lorentz force on electrical charges in the electrolyte [127, 128, 129], generating convection in the electrolyte. The convection can enhance mass transfer, during which Ohmic losses, and concentration overpotentials can be reduced [127, 128, 129]. The (convective)flow improved the desorption of bubbles and therefore improved the void fraction [130, 131, 132].

Reduced overpotentials have been measured as a result of applying a magnetic field (up to $1.5 \mathrm{~T}$ ) perpendicular to the working electrode [133]. The study employed frequency analysis of the noise spectrum in a system where hydrogen gas evolution from a platinum electrode ( $0.5 \mathrm{~mm}$ diameter) took place. The magnetic field decreased the overpotential for hydrogen formation by $10 \%(\sim 0.2 \mathrm{~V})$ for a field strength of $1.5 \mathrm{~T}$ by inducing electrohydrodynamic flow. It was stated that the measured overpotential decrease of $10 \%$ is similar to levels achieved with mechanical agitation.

The electrolysis efficiency for various electrode materials has been studied [128]. The electrolysis efficiency was found higher with the use of ferromagnetic materials than with paramagnetic material which in turn had a higher efficiency than diamagnetic materials. The electrode materials used were nickel, platinum, and graphite, respectively. The material performance was attributed to the intensity of spontaneous magnetization of the electrodes. Furthermore, it was found that a shorter interelectrode distance $(2 \mathrm{~mm})$ resulted in more significant effects (current densities of $170 \mathrm{~mA} / \mathrm{cm}^{2}, 35 \mathrm{~mA} / \mathrm{cm}^{2}$, and $24 \mathrm{~mA} / \mathrm{cm}^{2}$ were obtained form nickel, platinum, and graphite electrodes, respectively) caused by the magnetic field.

Ultrasound has been employed to increase mass transfer [134] and to actively remove bubbles from electrodes during reactions [134, 135, 136]. The influence of ultrasound on the overpotential of hydrolysis was studied [135]. A reduction in the cell voltage (the entire potential applied to operate the electroysis cell) was measured when ultrasound was applied, due to electrode polarization improvements by induced convection as well as a void fraction reduction. The effect was more pronounced under low electrolyte concentration and high current density conditions, because of the increased voltage. An improvement in hydrogen evolution of 5-18\% 
and energy savings up to $10-25 \%$ were reported.

\subsection{Conclusions}

There is economic interest for hydrogen gas to replace the depleting natural oil and gas reserves. The use of renewable energy sources such as solar energy to generate hydrogen gas from water has an additional benefit over many other solutions, that no $\mathrm{CO}_{2}$ is released upon water splitting nor at recombination. However, many challenges need to be overcome in hydrogen gas storage and distribution before hydrogen gas can be implemented at large scale. A main focus point is also the efficiency decrease by the presence of bubbles in electrolyzers. This problem needs to be addressed on the mesoscale where heat and mass transfer are enhanced and retention times are short such that the total overpotential can be lowered and Faradaic efficiencies increased.

Electrode materials can be chosen such that the activation overpotentials can be lowered in the specific environment in which the electrolyzer needs to operate. Electrolyzer design such as the electrode size, spacing, positioning, and membrane placement determines a large fraction of the resistance overpotential encountered. Membraneless solutions allow for novel electrolyzer designs in which the resistance overpotential arising from interfaces can be drastically reduced. Gas bubbles affect both resistance and concentration overpotentials, and are therefore of special interest. The presence of bubbles increases electrode screening and void fraction, at the same time the depletion of the gas concentration by a bubble can lower the concentration overpotential.

The nucleation of bubbles occurs typically heterogeneously on gas evolving electrodes due to the lowered thermodynamic energy barrier. However, cases in which homogeneous nucleation occurred have been reported. In one study homogeneous nucleation occurred due to strong electric fields in the electric double layer reducing the surface tension locally and with it the nucleation energy barrier. Also, homogeneous nucleation was reported when the wettability of the electrode did not accept the bubble nucleus readily. A mismatch between the classical nucleation theory and their experimental counterparts exists arising from surface tension effects often not being accounted for. The growth of bubbles is typically governed by the availability of gases, where diffusion governed growth of bubbles results in the bubble radius scaling with $\sqrt{t}$ and in reaction governed growth follows the bubble radius scaling with $\sqrt[3]{t}$. The growth of bubbles is often followed by detachment, analogous in boiling and electrolysis studies for low current densities such that the wettabillity of the electrode does not change. The influence of electric charge on the bubble detachment is a 
result of the electrodynamic interaction between charge residing on the bubble interface and the electrodes. A positively charged bubble interface is found at low $\mathrm{pH}$ where at high $\mathrm{pH}$ a negative charge is experienced. The addition of charged molecules to the interface showed influence of the electrodynamic interactions between the bubbles and electrodes. Next to the increased overpotentials caused by evolving bubbles in electrolyzers, losses in incident light by the presence of bubbles on photo-electrodes are the result of different pathways taken by the light through the bubble.

We consider that more research is needed for establishing better design rules for electrodes that can contribute to higher efficiencies, and an efficient deployment of the scientific knowledge to society in the form of commercial equipment. Despite the large number of literature reviewed, we believe that with the recent development of advanced imaging techniques (fast cameras) and cleverly designed experiments, the scientific community devoted to electrochemical studies involving bubble formation has still much to discover and learn.

\section{Outlook}

Determining by how much reaction efficiencies are lowered due to the presence of bubbles allows for a careful consideration in applying methods that can reduce the time bubbles are present in electrolyzers. Such methods are often paired with energy consumption themselves, such is most often the case when imposing electrolyte flow. Therefore, optimizations can be made in terms of the total energy required. Passive methods to displace or control the bubble onset formation are therefore of great importance.

How bubbles affect the resistive and concentration overpotentials is not well known. Small electrodes, such as nano- and micrometer sized electrodes, have been studied to a small extend. The larger electrodes, meso- and macroscale electrodes, provide a relatively unexplored area since effects as potential drops average out over large electrode surfaces. The covered works investigating resistive and concentration overpotentials made a good start on the subject, however it was required that several assumptions were made such as static bubble growth. Further research on the subject employing a non stagnant boundary layer and non static bubble growth has previously been proposed. Maragoni flow warrants further investigation as well, the decoupling of the thermal and concentration effects could perhaps be realized using large electrode surfaces where isolated successive bubble growth takes place. With these electrodes significant amounts of gas can be produced at low current densities while Joule heating could be minimized. 


\section{Acknowledgments}

This work was supported by the Netherlands Centre for Multiscale Catalytic Energy Conversion (MCEC), an NWO Gravitation programme funded by the Ministry of Education, Culture and Science of the government of the Netherlands. 


\section{3}

\section{Gas bubble evolution on microstructured silicon substrates}

The formation, growth and detachment of gas bubbles on electrodes are omnipresent in electrolysis and other gas-producing chemical processes. To better understand their role in the mass transfer efficiency, we perform experiments involving successive bubble nucleations from a predefined nucleation site which consists of a superhydrophobic pit on top of a micromachined pillar. The experiments on bubble nucleation at these spots permit the comparison of mass transfer phenomena connected to electrolytically generated $\mathrm{H}_{2}$ bubbles with the better-understood evolution of $\mathrm{CO}_{2}$ bubbles in pressure-controlled supersaturated solutions. In both cases, bubbles grow in a diffusion-dominated regime. For $\mathrm{CO}_{2}$ bubbles, it is found that the growth rate coefficient of subsequent bubbles always decreases due to the effect of gas depletion. In contrast, during constant current electrolysis the bubble growth rates are affected by the evolution of a boundary layer of dissolved $\mathrm{H}_{2}$ gas near the flat electrode which competes with gas depletion. This competition results in three distinct regimes. Initially, the bubble growth slows down with each new bubble in the succession due to the dominant depletion of the newly-formed concentration boundary layer. In later stages, the growth rate increases due to a local increase of gas supersaturation caused by the continuous gas production and finally levels off to an approximate steady growth rate. The gas transport efficiency associated with the electrolytic bubble succession follows a similar trend in time. Finally, for both $\mathrm{H}_{2}$ and $\mathrm{CO}_{2}$ bubbles, detachment mostly occurs at smaller radii than theory predicts and at a surprisingly wide spread of sizes. A number of explanations are proposed, but the ultimate origin of the spreading of the results remains elusive. ${ }^{1}$

\footnotetext{
${ }^{1}$ This chapter has been published as: van der Linde, P. Peñas-López, P. Moreno Soto, Á. van der Meer, D. Lohse, D. Gardeniers, H. and Fernández Rivas, D. Gas bubble evolution on microstructured silicon substrates, Energy Environ. Sci., 11, 3452-3462, 2018. DOI: 10.1039/C8EE02657B
} 


\subsection{Introduction}

Hydrogen is a promising energy carrier that can be obtained via zero $\mathrm{CO}_{2}$ emission techniques $[8,137,138]$ such as solar-driven water splitting [139, $140,141,142]$. However, the chemical reactions involved in such processes result in bubble generation. Such bubbles can block the reacting surfaces and decrease the process efficiency $[116,126]$.

The formation of bubbles on liquid-immersed surfaces is relevant for many gas-producing processes such as boiling [143], catalysis [144, 145] and electrolysis [85, 146]. More specifically, the formation of bubbles during chemical processes may be either beneficial due to increased heat and mass transfer induced by convection upon bubble detachment [147], or detrimental due to overpotentials caused by blocked active sites on the electrodes [148, 69, 149].

Bubbles preferably nucleate in small defects such as pits or crevices, where gas can be easily entrapped and the energy barrier is smallest [13]. A certain control over the location at which bubbles are prone to nucleate can be achieved by modifying the topography of the solid surface with suitable microstructures that act as preferential nucleation sites. The robustness of this concept has been demonstrated during pressure pulse propagation [150], ultrasound exposure [151], turbulent boiling [152] and under liquid flow conditions [29]. For this purpose, pillars are fabricated as preferential nucleation sites for bubbles, as shown in Figure 3.1D, following a long-term line of research in our group with the aim of understanding and controlling the bubble evolution as a function of gas diffusion [153, 113, 103, 28].

Three different phases can be distinguished during bubble evolution as shown in Figure 3.1: bubble nucleation at the surface (Figure 3.1A), growth (Figure 3.1B) and detachment (Figure 3.1C). In this study, we provide an in-depth comparative analysis between bubble evolution on a single pillar during electrolysis and the better-understood bubble evolution in pressure-controlled $\mathrm{CO}_{2}$ supersaturated solutions on the same geometry, working out similarities and differences between the two processes. Our ultimate goal is to increase energy conversion efficiencies of solar-driven water splitting systems by controlling the gas bubble evolution on micromachined electrodes. 
A) Nucleation

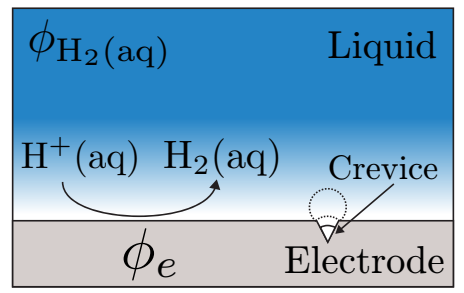

B) Growth

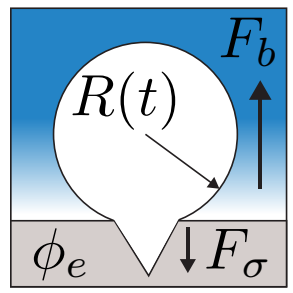

C) Detachment

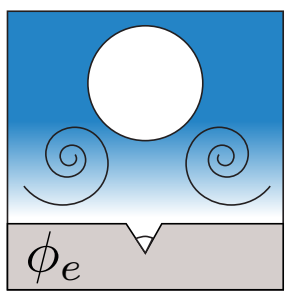

D) Artificial nucleation
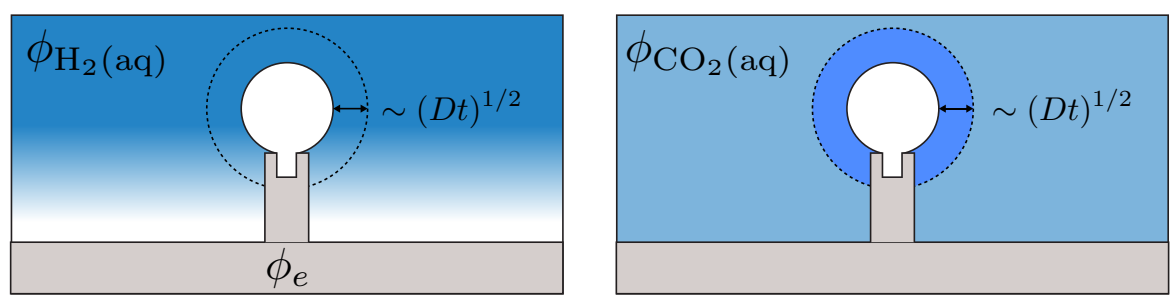

Figure 3.1: Various stages of bubble evolution on electrodes. A) Heterogeneous bubble nucleation, here shown to occur in a crevice. The electron flux towards the electrode surface is indicated by $\phi_{e}$. The flux $\phi_{\mathrm{H}_{2}(\mathrm{aq})}$ indicates the diffusive transport of $\mathrm{H}_{2}$ gas to the nucleating bubble. The highest gas concentration is at the electrode surface, indicated by a lighter blue color (the same colour pattern applies to the other plots). B) Bubble growth on the electrode surface. The direction of the interfacial tension force $F_{\sigma}$ and buoyancy force $F_{b}$ are shown. C) Detachment of bubbles by buoyancy overcoming the interfacial tension force which pins the bubble to a crack or crevice. D) Artificial nucleation sites to facilitate successive bubble evolution. On the left panel, the $\mathrm{H}_{2}$ bubble evolution during water splitting is shown. The dotted area shows the time-dependent area from which the bubble experiences influx of gas via diffusion. On the right panel, the $\mathrm{CO}_{2}$ bubble evolves in a $\mathrm{CO}_{2}$ supersaturated medium. The gas concentration is homogeneous in the liquid apart from the time-dependent area around the bubble where the gas becomes depleted as successive bubbles grow [103], indicated by a darker blue color. 


\subsubsection{Outlook}

In this fundamental study, we have investigated the isolated bubble evolution on artificial nucleation sites micromachined on electrodes. The knowledge achieved with our experimental and theoretical work can certainly assist in the design of novel devices in the future. These future works could use nucleation sites to prevent the crossover of species in configurations in which the electrodes could be used to drive the bubbles to different streams [66] or to facilitate buoyancy driven separation mechanisms [65]. Artificial nucleation sites could also be used to evolve bubbles in predefined locations, a scenario which has been suggested to give rise to increased flexibility in device design, optimization and operation [6]. The use of multiple nucleation sites on electrodes permits the definition of areas on the electrodes where bubbles are generated such that they do not compete for evolved gas as well as areas where they do. This could determine areas on the electrode surface where bubbles do not form and dedicated areas where bubbles do form, allowing for controlled bubble formation at higher current densities. Major advantages could lie in designing electrodes where the catalytic surface is kept free from bubbles.

\subsection{Materials and methods}

\subsubsection{Microfabrication of silicon substrates}

Micropillars on the surface of the electrode increase the active area and contact with the liquid phase, ultimate characteristics which are desirable in photolysis applications [154, 155]. This approach encourages the construction of small and dense structures which work as light-harvesting areas. With the aim of understanding the fundamentals of bubble evolution on pillars, we focus on a single pillar microstructure of radius $R_{p}=2.5-$ $15 \mu \mathrm{m}$ to study the succession of single bubbles generated on them. A superhydrophobic pit on top of the micropillar serves as the nucleation site [13].

Boron-doped silicon wafers with (100) crystal orientation, resistivity in the range of $0.01 \Omega \cdot \mathrm{cm}-0.025 \Omega \cdot \mathrm{cm}$, thickness of $525 \mu \mathrm{m}$ and single side polished, were covered by $1.7 \mu \mathrm{m}$ Olin OiR 907-17 resist. Using photolithography, circular regions ranging $R_{0}=1-10 \mu \mathrm{m}$ in radius were defined, as shown in step 1 in Figure 3.2D. The circular regions were etched with a deep reactive ion etching (DRIE) Bosch process (Adixen AMS100SE) to a depth of $\sim 20 \mu \mathrm{m}$. Black silicon was formed at the bottom of the pits with DRIE, as shown in step 2 in Figure 3.2D. Black silicon is an important structure that allows for better gas trapping while immersing the 
substrates in liquid. Afterwards, additional fluorocarbons were deposited ( $\pm 40 \mathrm{~nm} / \mathrm{min}$ ) inside the pits, turning them superhydrophobic [156]. The deposition times varied per set of samples between $7 \mathrm{~s}$ to $60 \mathrm{~s}$.

The pillar radii were defined with photolithography as shown in step 3 in Figure 3.2D. These pillars were etched with DRIE to various heights in the range of $0 \mu \mathrm{m}-60 \mu \mathrm{m}$. An aluminium contact was created via DC-sputtering with a thickness of $100 \mathrm{~nm}(99 \% \mathrm{Al}, 1 \% \mathrm{Si})$ at the bottom of the substrate, as shown in step 4 in Figure 3.2D. An ultrasound (VWR Ultrasonic Cleaner USC-THD, $45 \mathrm{kHz}$ ) acetone bath was used to remove the resist. Afterwards, the wafers were diced (Disco DAD 321) into 10 $\mathrm{mm} \times 10 \mathrm{~mm}$ square substrates. Prior to the measurements, the samples were cleaned with another ultrasound acetone bath step. Figure 3.2A-B shows SEM images of fabricated micropillars and Figure 3.2C shows the black silicon inside the superhydrophobic pit.

\subsubsection{Experimental set-ups for bubble evolution}

Figure 3.3 shows the electrolysis set-up, consisting of a custom-made acrylic holder, a camera and a power source. The acrylic holder is designed to keep the substrate in place, to hold a platinum wire counter electrode far away from the growing bubble and to contain the electrolyte. A circular area of the silicon substrate with radius $R_{e}=3.5 \mathrm{~mm}$ and sealed to the holder with a Teflon ring is in contact at all times with the electrolyte. This radius is approximately ten times the maximum bubble radius and, therefore, we can assume that the holder walls do not play any significant role during bubble growth on the pillars. The substrate contains an electrical contact at the bottom aluminium layer through which the current is supplied (not shown in Figure 3.3 for simplicity). A Keithley 2410 power source is used to drive the constant-current electrolysis. For optical imaging, a Flea ${ }^{\circledR} 3$ Monochrome Camera, (optical resolution of $1.1 \mu \mathrm{m} / \mathrm{pixel}$ ) is coupled to a 50/50 Beam-splitter Cube. For illumination, a Galvoptics KL2500 LCD 230V light source is used.

At the beginning of each experiment, the holder is filled with $20 \mathrm{~mL}$ of fresh electrolyte. The electrolyte consists of a solution of non-degassed Milli-Q water with $10 \mathrm{mM} \mathrm{Na}_{2} \mathrm{SO}_{4}$ salt and a pH 3 buffer of $1 \mathrm{mM}$ anhydrous sodium acetate and $0.1 \mathrm{M}$ acetic acid. The temperature remains constant at all times, $T \approx 20^{\circ} \mathrm{C}$. During each experiment, a constant current in the range of $10 \mu \mathrm{A}-600 \mu \mathrm{A}$ is supplied. The resulting current density $J$ falls in the range of $0.3 \mathrm{~A} / \mathrm{m}^{2}-15 \mathrm{~A} / \mathrm{m}^{2}$. The potentials during experiments were measured within a range of $1.8 \mathrm{~V}$ to $4.9 \mathrm{~V}$.

To compare the evolution of $\mathrm{H}_{2}$ bubbles generated by electrolysis with that of $\mathrm{CO}_{2}$ bubbles growing in an initially uniformly supersaturated 


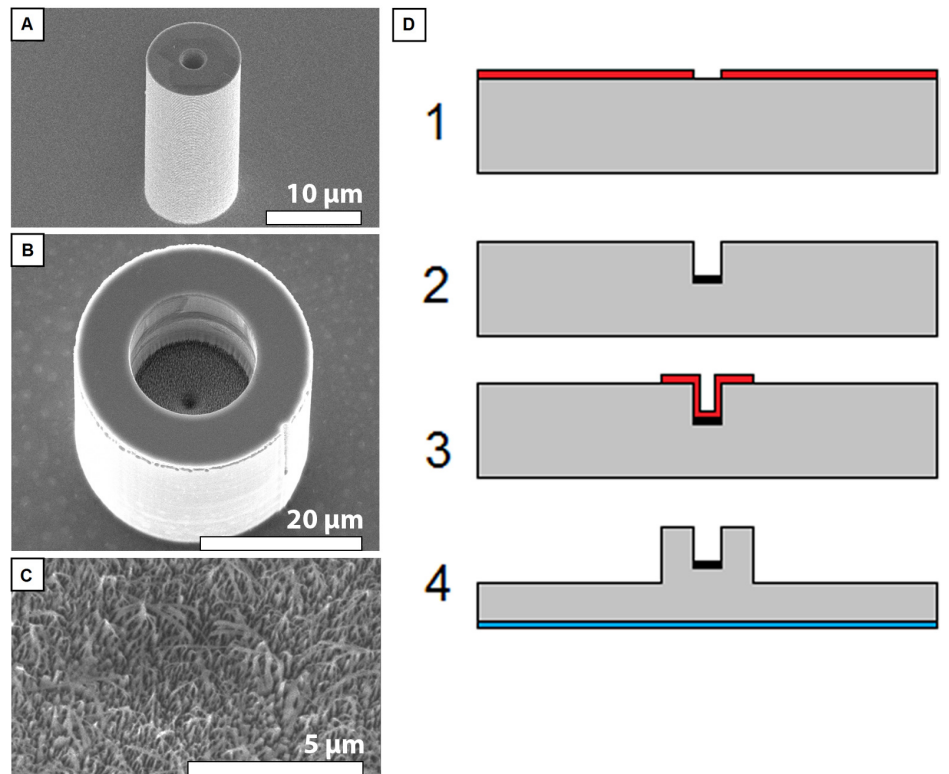

Figure 3.2: Scanning Electron Microscope (SEM) images of A) a micropillar with a $10 \mu \mathrm{m}$ diameter, a pit diameter of $2 \mu \mathrm{m}$ and a pillar height of 25 $\mu \mathrm{m}$, viewed at a $45^{\circ}$ angle, B) a micropillar with a $30 \mu \mathrm{m}$ diameter, a pit diameter of $15 \mu \mathrm{m}$ and a pillar height of $30 \mu \mathrm{m}$ viewed at a $20^{\circ}$ angle, and C) a close-up of black silicon at the bottom of the pit in panel B viewed under a $20^{\circ}$ angle. D) Sketch of the cross-sectional view (not to scale) of the substrate fabrication process. Step 1 shows the $\mathrm{p}^{++}$type silicon wafer on which a pattern is created via photolithography to mark the outline of the pit. With dry etching, a pit is created and black silicon formed at its bottom, step 2. Resist is applied and patterned via photolithography to mark the outline of the micropillar for dry etching, step 3, after which an aluminium backside contact is formed via DC sputtering. The resulting complete substrate is shown in step 4. 


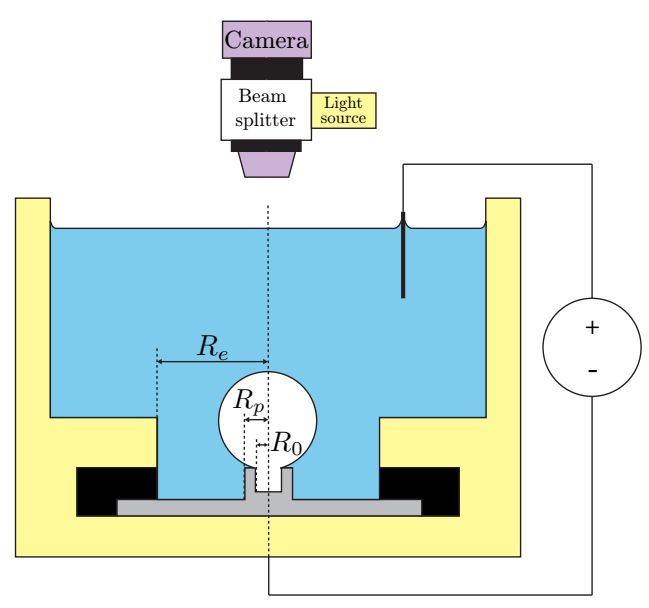

Figure 3.3: Schematic of the electrolysis set-up (not to scale). At the top, the optics consist of a camera, lens and light source. Below the optics, an acrylic holder (yellow) which contains the substrate (grey) is placed. A circular area of the substrate of radius $R_{e}=3.5 \mathrm{~mm}$ is in contact with the electrolyte (light blue), in which the counter electrode is placed (top right). A DC power source is used to drive the reaction.

solution, identical silicon substrates are placed within a pressurized test chamber (pressure $P_{0} \approx 9$ bar) that is filled with carbonated water previously saturated at the same pressure. By lowering the pressure to approximate values of $P_{l} \approx 7.7$ bars, a supersaturation of $\zeta=P_{0} / P_{l}-$ $1 \approx 0.17$ is achieved following Henry's law (at constant temperature) and, consequently, bubbles nucleate and grow on the predefined spots. A detailed description of this experimental set-up and procedure can be found elsewhere [153, 103].

\subsection{Results and discussion}

\subsubsection{Bubble nucleation on a superhydrophobic pit}

The superhydrophobic pit entraps a gas pocket upon submersion in a liquid [150] and hence acts as a site for heterogeneous nucleation. The interfacial or equilibrium concentration of dissolved gas at a liquid-gas interface can be written as $C=k_{H} P_{g}$ according to Henry's law, where $k_{H}$ is Henry's constant (a decreasing function of temperature specific to each gas-liquid pair) and $P_{g}$ is the partial pressure of the gas acting on the liquid surface [157]. For a pit with a circular opening of radius $R_{0}$, the pressure 
threshold at which bubbles begin to grow is given by the condition [156]

$$
P_{v}+P_{g}>P_{l}+\frac{2 \sigma}{R_{0}} \equiv \frac{P_{v}}{P_{l}}+(\zeta+1)>1+\frac{2 \sigma}{P_{l} R_{0}},
$$

where $P_{v}$ is the liquid vapor pressure, $P_{l}$ is the liquid pressure and $\sigma=0.07$ $\mathrm{N} / \mathrm{m}$ is the liquid-gas interfacial tension (for simplification, we assume a constant value for both $\mathrm{H}_{2}$ and $\mathrm{CO}_{2}$ cases). The radius $R_{0}$ in the Laplace pressure term (last term in (3.1)) is justified since, at the nucleation stage, the bubble can be assumed to be a hemispherical cap of radius $R_{0}$ growing from the pit with the same radius. Equation (3.1) reflects that the pressure inside the bubble must overcome the forces resulting from the liquid pressure and surface tension to achieve bubble growth. If a multicomponent solution of $N$ volatile ideal gas species is considered, the condition for growth in (3.1) can be approximated as [158]:

$$
\frac{P_{v}}{P_{l}}+\sum_{i=1}^{N}\left(\zeta_{i}+1\right)>1+\frac{2 \sigma}{P_{l} R_{0}},
$$

where $\zeta_{i}=C_{i} /\left(k_{H, i} P_{l}\right)-1$ is the supersaturation of the dissolved gas species $i$ (in general, position and time dependent), with $C_{i}$ being the gas concentration in $\mathrm{mol} / \mathrm{m}^{3}$. With this equation, we can calculate the critical minimum supersaturation level required to overcome the energy barrier due to surface tension.

For the electrolysis case, we perform experiments at $T=20^{\circ} \mathrm{C}$ and $P_{l}=$ 1 bar. Under these conditions, the water vapour pressure can be neglected since $P_{v} / P_{l} \sim 0.02$ (the effect of dissolved gases on the vapour pressure has been considered negligible since their mole fraction is small enough to assume that there is no appreciable change in the boiling point of water). $\mathrm{H}_{2}$ gas bubbles grow in a binary solution of $\mathrm{H}_{2}$ and air since the electrolyte is not degassed (this condition is similar to that present in real electrolyzer applications) and it is permanently exposed to ambient air throughout its preparation, subsequent storage and finally during experiments. Therefore, it is reasonable to assume that it is air equilibrated, i.e. $\zeta_{\text {air }}=0$ (assuming that air is a single component entity). Consequently and according to (3.2), the minimum supersaturation of $\mathrm{H}_{2}$ required to trigger growth for a typical pit radius $R_{0}=2.5 \mu \mathrm{m}$ corresponds to

$$
\zeta_{H_{2}}=\frac{2 \sigma}{P_{l} R_{0}}-1 \approx-0.44 .
$$

In practical terms, the negative value above means that the presence of other dissolved species, i.e. air (which consists of a mixture of $\mathrm{N}_{2}, \mathrm{O}_{2}$ and other gases), makes bubble nucleation easier and, consequently, it is 
possible to achieve bubble nucleation shortly after initiating the electrolysis. We can anticipate that somewhat higher concentrations are required in practice. There are many other factors that can inhibit bubble nucleation and growth. Those will be discussed later in the text.

In contrast, the experiments with $\mathrm{CO}_{2}$ bubbles growing from pressure-controlled supersaturated carbonated water within a pressurized chamber are performed at a liquid pressure $P_{l} \approx 7.7$ bars and isolated from the outside. The preparation procedure ensures that in the experimental chamber there are no other gas species present within the liquid apart from $\mathrm{CO}_{2}$. Therefore, the minimum supersaturation required for nucleation is

$$
\zeta_{\mathrm{CO}_{2}}=\frac{2 \sigma}{P_{l} R_{0}} \approx 0.07 .
$$

Note that in this case a positive minimum supersaturation value is necessary. Supersaturation levels below $\zeta_{\mathrm{CO}_{2}}=0.07$ were tried and resulted in no bubble generation. The lowest $\mathrm{CO}_{2}$ supersaturation for which we experimentally achieved bubble growth was indeed $\zeta_{\mathrm{CO}_{2}} \approx 0.07$.

\subsubsection{Bubble nucleation times}

In constant-current electrolysis and in the absence of bubbles, the (molar) concentration of $\mathrm{H}_{2}$ near the electrode can be estimated as

$$
C(t)=\frac{2 J}{F z \sqrt{\pi D}} \sqrt{t},
$$

which is an increasing function of time obtained by solving the 1D diffusion equation in a semi-infinite domain with a constant flux boundary condition $[159,28]$. Here, $t$ denotes the time after the start of electrolysis, $J$ is the current density, $z=2$ is the valency of the $\mathrm{H}_{2}$ evolution reaction, $F=96485 \mathrm{C} / \mathrm{mol}$ is Faraday's constant and $D=4.5 \times 10^{-9} \mathrm{~m}^{2} / \mathrm{s}$ is the diffusivity of $\mathrm{H}_{2}$ in water. Combining Henry's law, (3.3) and (3.5), we obtain the theoretical minimum time for a bubble nucleation after the start of electrolysis as a function of the current density:

$$
t^{*}=\frac{\pi \sigma^{2} k_{H, \mathrm{H}_{2}}^{2} F^{2} z^{2} D}{J^{2} R_{0}^{2}} .
$$

Here $k_{H, \mathrm{H}_{2}}=7.7 \times 10^{-6} \mathrm{~mol} / \mathrm{N} \cdot \mathrm{m}$. It stands to reason that as $J$ increases, the gas formation rate also increases and, therefore, the minimum time to nucleate a bubble is achieved faster. There is evidence that the concentration at which the first bubble nucleates on a gas-evolving electrode also depends on the value of the current density [159]. Tawfik et al. (2014) reported that the nucleation time does not depend on a 
constant concentration $C$, but rather on the applied current density $J$, with $C$ increasing as $J$ increases. They proposed the following empirical relation for the nucleation time of the first bubble spontaneously growing on a flat electrode in a presumably non-degassed electrolyte:

$$
t^{*}=k \pi z^{2} F^{2} D J^{-1},
$$

with $k=0.19 \mathrm{~mol}^{2} / \mathrm{m}^{4} \mathrm{~A}$ a fitting constant. The nucleation times of the first $\mathrm{H}_{2}$ bubble in the succession on the predefined pits are plotted vs the current density in Figure 3.4 and compared to the theoretical prediction in (3.6) with $R_{0}=5,7.5$ and $10 \mu \mathrm{m}$, and the empirical relation in (3.7). The times are measured from the start of the electrolysis up to a threshold radius of $\sim 25 \mu \mathrm{m}$, following the method used by Tawfik and Diez [159]. The nucleation time, $t^{*}$, appears to generally decrease with $J$; however, no clear trend can be appreciated. The significant variability in our experimental measurements can be attributed to three possible causes:

(1) The pit topography is different from sample to sample. We measured deviations from the ideal circular pit opening in the radial direction of several hundreds of nanometers (refer to the Electronic Supplementary Information). Fluorocarbons within the pit may hinder mass transport of dissolved gas towards the gas pocket or enforce pinning at different contact angles and, thus, affect the effective value of $R_{0}$.

(2) The current density is likely far from being spatially uniform along the electrode surface $[160,28]$.

(3) The electrolyte contains air, partially composed of $\mathrm{O}_{2}$. Oxygen reduction competes with $\mathrm{H}_{2}$ formation. This implies that the net current density available for $\mathrm{H}_{2}$ formation is less than the actually applied current density. By definition, the standard potential for $\mathrm{H}_{2}$ formation is $0 \mathrm{~V}$, whereas $\mathrm{O}_{2}$ reduction occurs at $0.40 \mathrm{~V}$. Consequently, higher current densities result in both more $\mathrm{H}_{2}$ production and $\mathrm{O}_{2}$ reduction. This fact means that $\mathrm{H}_{2}$ is not efficiently produced (not all of the applied current is used for its generation) and, thus, the bubble nucleation time seems not to follow a clear decreasing trend with increasing $J$. Furthermore, this may be a cause of the scattering in Figure 3.4, since $\mathrm{O}_{2}$ levels at the start of each experiment may not be the same (although a fresh solution was employed for each experiment). The levelling off of the nucleation time at higher current densities in the same figure could be attributed to the influence of the dissolved $\mathrm{O}_{2}$ reduction, the unequal distribution of gas production, the time required for the diffusion of the gases through the liquid 
towards the artificial nucleation site and the stochastic nature of nucleation. In addition to the influence of the parameters mentioned above, other factors unknown to us may play a rather significant role in the measured deviation between the nucleation times of the bubbles and of the predicted theoretical values.

Moreover, the empirical prefactor in (3.7) may correct for the growth of the bubble to the threshold size of $25 \mu \mathrm{m}$, even though the time needed to reach that threshold may be negligible compared to the time necessary to achieve nucleation. However, no such correction is performed in (3.6). Surface tension reduction due to dissolved gases in the solution can also explain why the experimental nucleation times differ from those predicted by theory [88]. For electrolysis, the nucleation times for the various applied currents in this research fall within the order of tenths of seconds. In comparison, the nucleation of $\mathrm{CO}_{2}$ bubbles in carbonated water is observed to occur at or below the order of seconds after the pressure was reduced below the saturation value [103]. The differences may rely then on the different ways of bubble generation and not on the substrate surface properties.

\subsubsection{Bubble growth}

Bubble growth can be described as $R(t) \propto t^{\alpha}$, with $R$ denoting the bubble radius, $t$ the time after nucleation and $\alpha$ the time exponent [92]. For diffusive bubble growth, $\alpha=1 / 2$ [14], whereas for reaction limited growth $\alpha=1 / 3$ [104, 92].

In electrolysis, diffusion-limited growth occurs when the characteristic time of the diffusive transport of the evolved gas across the electrode, $t_{e} \approx R_{e}^{2} / D$ (where $D$ is the diffusion coefficient), is much larger than that of the diffusive gas transport to the bubble, $t_{d} \approx R_{d}^{2} / D$. The relation between these two characteristic diffusive times can be associated to the Damköhler number, which is defined as $D a=t_{e} / t_{d}=R_{e}^{2} / R_{d}^{2}$ and can be interpreted as the ratio of the diffusive transport across the characteristic electrode size and across the characteristic bubble size. Here, $R_{e}=3.5 \mathrm{~mm}$ is the electrode radius and $R_{d} \sim 0.3 \mathrm{~mm}$ is the bubble experimental mean detachment radius of all experiments at all current densities, which results in $D a \approx 100$. Therefore, our research focuses on bubble growth during electrolysis controlled by diffusion.

Figure 3.5A and 3.5B (top plot) show the evolution of bubble radii over time of five series of successive $\mathrm{H}_{2}$ bubbles produced at constant-current electrolysis. Each series corresponds to a different current density. At the beginning, each successive bubble evolves slower than the previous one approximately up to the $4^{\text {th }}$ bubble, when the growth rate becomes faster. 


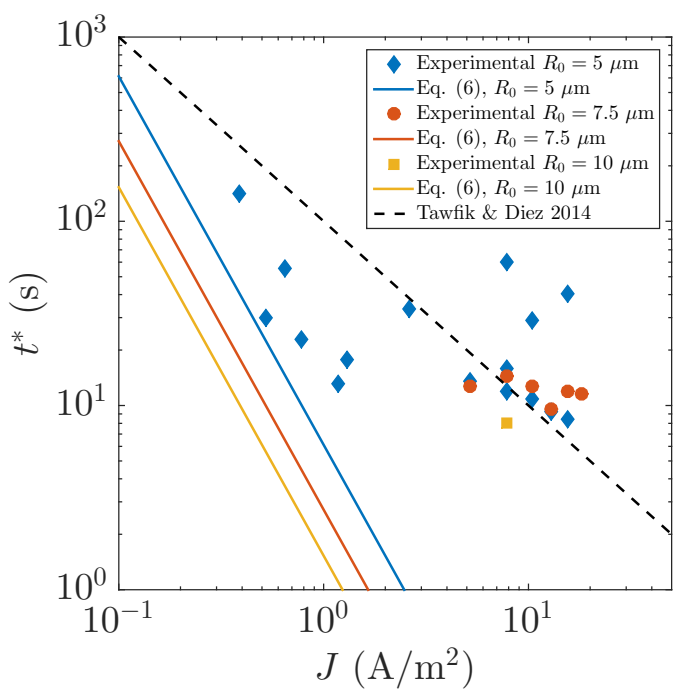

Figure 3.4: Experimental nucleation time of the first $\mathrm{H}_{2}$ bubble formed since the start of electrolysis as a function of current density. The blue diamonds, red circles and yellow squares show the measured nucleation times with $R_{0}=5,7.5$ and $10 \mu \mathrm{m}$, respectively. The blue, red, and yellow lines represent Equation (3.6) for $R_{0}=5,7.5$ and $10 \mu \mathrm{m}$, correspondingly. The dashed line shows the empirical relation by Tawfik and Diez (3.7) [159]. Generally, the nucleation time $t^{*}$ decreases with current density $J$. Discrepancies between experiments and the theoretical prediction are more than apparent and explained in the text. 


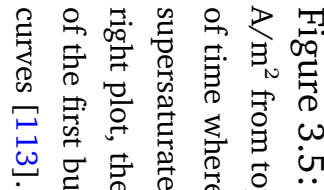

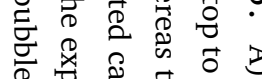

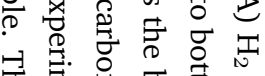

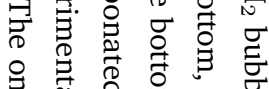
号壱芯

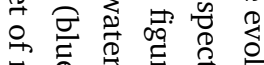
帝

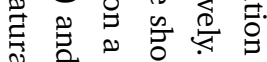

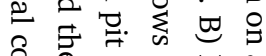
吕京志志 ก

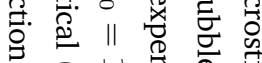
。

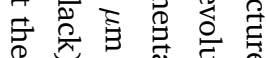

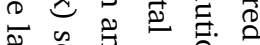

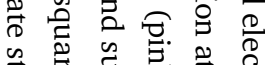

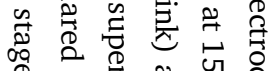

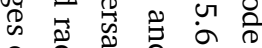

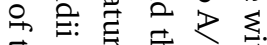

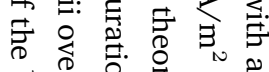
용요

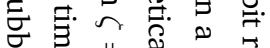

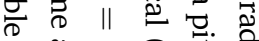
o0

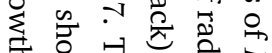

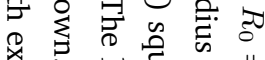

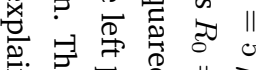

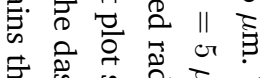

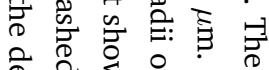

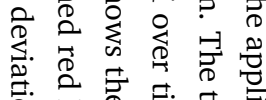

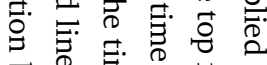
范

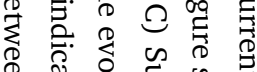
๑节完苛帘

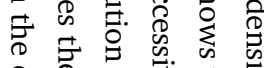
胥

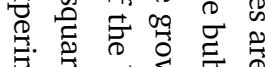

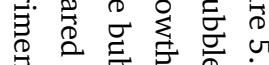
$\exists$ 西 5 i

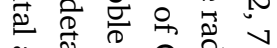

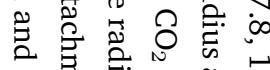

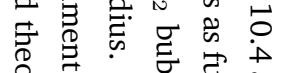
虽

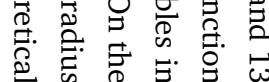
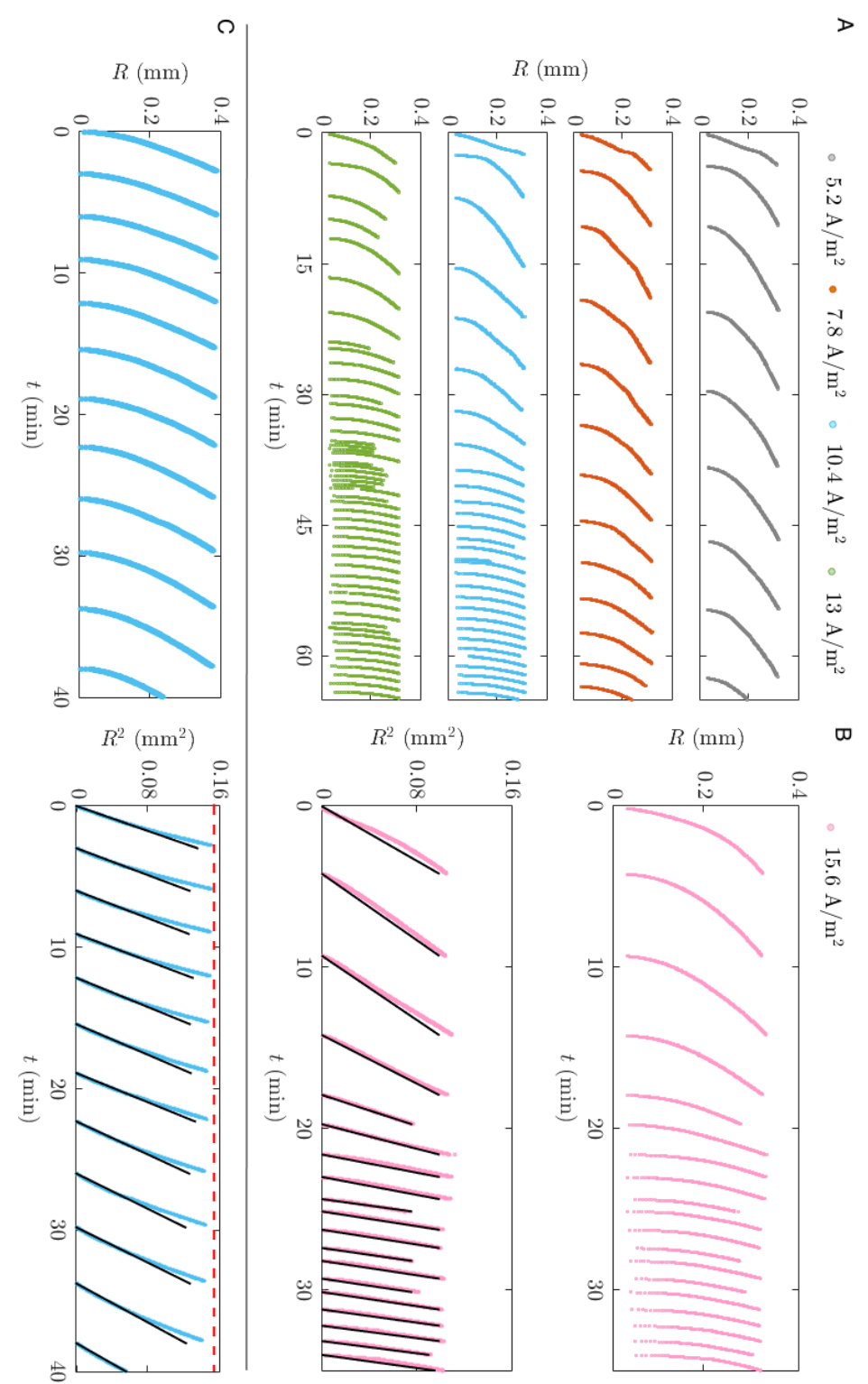
This acceleration is attributed to the evolution of the diffusive concentration boundary layer in which the bubbles grow [28] and the most-probable complete reduction of the dissolved $\mathrm{O}_{2}$ in the electrolyte (see item (3) in the discussion above). With increasing current densities, the growth rates at the beginning of each succession increase due to the larger gas production, but the evolution trend remains unaltered since the early bubbles in the succession deplete the diffusive concentration boundary layer around them.

The unsteady nature of the electrolytic bubble growth becomes more apparent upon comparison with the bubble growth in pressure-controlled supersaturated carbonated liquid (Figure 3.5C). In this figure, we present a succession of $\mathrm{CO}_{2}$ bubbles in supersaturated water at $\zeta=0.17$. The growth in this case continuously slows down with the successive bubble detachment due to the active depletion of the total amount of $\mathrm{CO}_{2}$ gas available [103]. In contrast, for the electrolytically-generated bubbles, after the early depletion the $\mathrm{H}_{2}$ gas concentration near the substrate continues to increase over time due to the continuous water splitting reaction resulting in a faster growth of the $\mathrm{H}_{2}$ bubbles [28].

Both $\mathrm{H}_{2}$ and $\mathrm{CO}_{2}$ bubbles evolve via pure diffusive growth, namely

$$
R(t)=\tilde{b} \sqrt{D t},
$$

where $\tilde{b}$ is the dimensionless growth coefficient [28]. The straight slopes observed in $R^{2}$ plotted against time in Figures 3.5B (bottom plot) and C (right plot) corroborate this behaviour. A short movie showing a succession of single $\mathrm{H}_{2}$ bubbles during electrolysis can be found on-line along this article (Movie 1).

The gas boundary layer evolution during electrolysis results in three different growth regimes, which are further elucidated by taking a closer look at the growth coefficient $\tilde{b}$. Figure 3.6 shows the evolution of $\tilde{b}$ with time since the start of electrolysis calculated from the data in Figure 3.5A and the top plot in 3.5B. Note that each experimental point corresponds to the growth coefficient of a particular bubble in the succession. Initially, $\tilde{b}$ decreases as a consequence of the initial bubble locally depleting the boundary layer of gas, behaviour referred to as the 'stagnation' regime (I). Successive bubbles keep growing in a mildly supersaturated liquid until the boundary layer overcomes the depletion losses due to the constant gas production and evolves to higher gas concentrations. The accompanying increase in $\tilde{b}$ characterises regime II, in which bubbles grow faster. The transition between regimes depends on the applied current density: the higher the current density, the earlier the onset of increasing $\tilde{b}$. Finally, regime III shows a stabilization in the growth rate for successive bubbles, reflected by $\tilde{b}$ increasing in small increments. In contrast, the growth coefficients corresponding to the $\mathrm{CO}_{2}$ bubble succession in Figure 3.5C 
always decrease due to gas depletion, [103] inset in Figure 3.6, similar to the early $\mathrm{H}_{2}$ bubbles in electrolysis (regime I). In this case, there is no influx of new gas which can counteract this depletion effect, resulting in a continuous smaller growth rate. The pillar height does not have any influence on the bubble growth coefficients [28]. For a more in-depth discussion on the different growth regimes and the influence that the boundary layer and its depletion have on the bubble growth dynamics, the interested reader is referred to van der Linde et al. (2017) [28].

During both $\mathrm{H}_{2}$ and $\mathrm{CO}_{2}$ measurements, successive bubble growth could suddenly stop because of spurious pit deactivation. This may occur once liquid enters the pit during bubble detachment: the interface of the gas pocket in the pit can form a jet which can wet the surface inside the pit, displacing the air [156]. We found no consistency in how long bubbles can be generated before pit deactivation. The fastest deactivation in the measurements occurred after the growth and detachment of a single bubble.

\subsubsection{Bubble detachment}

The position of the triple contact line on the pit-pillar microstructure and the contact angle dynamics determine the size at which the bubbles detach from the microstructure [161, 162]. Since optical access to the contact line was not possible, we speculate on five probable pinning positions during the bubble evolution process, sketched in Figure 3.7A. The inner surface of the pit contains several artifacts as a result of the fabrication process that can pin the bubble interface. As shown in Figure 3.7B, needle-like structures of black silicon are present at the bottom of the pit, whereas the inner surface contains vertical and horizontal scallops resulting from the Bosch etching process [163]. Additionally, the fluorocarbon (FC) layer deposited for enhanced hydrophobicity can facilitate pinning. Typically, the FC layer will adhere to the pit wall; however, in Figure 3.7B the layer detached prior to the FIB milling process (a video can be found on-line along this article) as observed with optical microscopy and SEM. This event could provide unpredictable pinning positions during the experiments and, consequently, end up in a different detachment radius. However, we have evidence that for the majority of the bubbles, the pinning is most likely to occur inside the pit (position I) throughout their whole lifetime, forming a bridging neck between the gas trapped in the pit and the bubble growing outside [103]. 


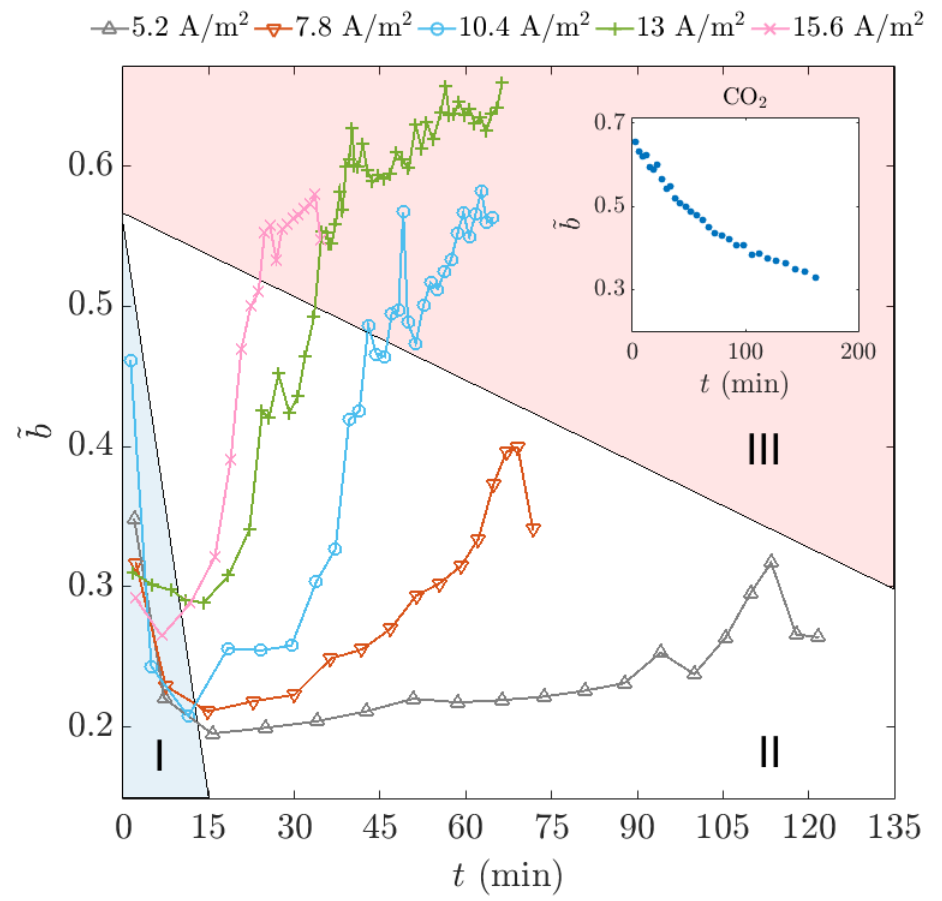

Figure 3.6: The dimensionless growth coefficient $\tilde{b}$ per successive $\mathrm{H}_{2}$ bubble as function of the time after the start of the electrolysis, $t$. The data are derived from the experimental results in Figure 3.5A and the top plot in Figure 3.5B. The different regimes are marked by the shaded regions, where region I corresponds to the stagnation regime in which $\tilde{b}$ decreases due to the early depletion, region II shows the counteracting effect due to the continuous gas production and III marks the regime in which an approximated steady state is reached. The transition between regimes II and III is defined by the moment in which the derivative $d \tilde{b} / d t$ drastically decreases, i.e. $\tilde{b}$ approaches a quasi-steady state. With increasing current density, the transition between regimes occurs faster because of the increased gas formation and the faster bubble evolution and their corresponding boundary layer. The inset shows the single regime I for the $\mathrm{CO}_{2}$ bubble growth coefficient in supersaturated carbonated water caused by continuous depletion due to the successive bubble growth. 

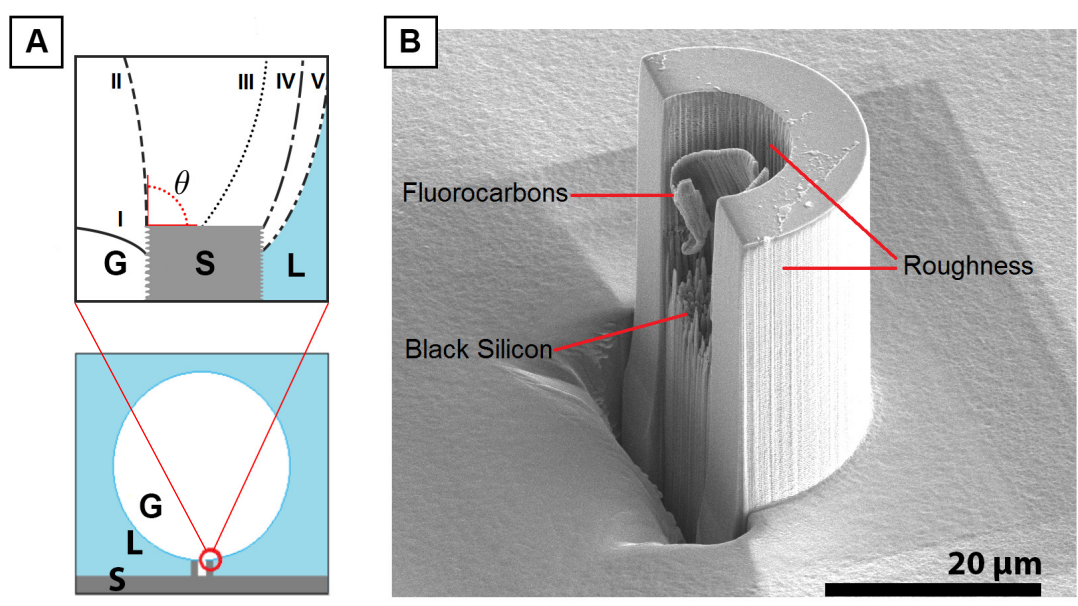

Figure 3.7: A) Schematic side view of a cross section showing a micropillar with micropit, indicating five possible pinning positions for the contact line of evolving bubbles. Position I is inside the pit, II is at its edge with an arbitrary contact angle $\theta$ shown in red, position III indicates a transition location, IV the outer edge of the pillar and $\mathrm{V}$ is on the pillar surface. B) Focused ion beam (FIB) milled down pillar, under $52^{\circ}$ angle with respect to the electron source. The inside of the pit shows the black silicon needle structure at the base and a detached fluorocarbon layer. Etching defects (vertical lines along the pillar) on the inside and on the outside are present. A video recording of the milling process can be found on-line along this article (Movie 2). 
As the bubble grows and attains its detachment size, it is possible that the bubble contact line moves from position I up to V [164], as sketched in Figure 3.7A. The departure size is an indirect way of estimating the position of the contact line. The maximum theoretical value of the bubble detachment radius growing from a pit of radius $R_{0}$ is given by Fritz's formula [114],

$$
R_{d}^{*}=\left(\frac{3 R_{0} \sigma}{2 \Delta \rho g}\right)^{1 / 3},
$$

with $\Delta \rho$ the difference in density between the liquid and gas phases and $g=9.81 \mathrm{~m} / \mathrm{s}^{2}$ the gravitational acceleration. Equation (3.9) can be derived from the balance between buoyancy and capillary forces, assuming that the contact line is at position II with a contact angle of $90^{\circ}$ with respect to the horizontal at the moment of detachment, as sketched in Figures 3.1B and 3.7A. Net charges present on bubbles due to the solvent $\mathrm{pH}$ or absorbed species, such as surfactants [91], may affect the pinning position of the bubble to the pit and consequently, its final detachment radius. Our electrolysis experiments are carried out in a medium with a pH 3 buffer and with no absorbent species to ensure a point of zero charge on the bubble. We can thus exclude electrostatic forces from the detachment force balance.

Figure 3.8 shows the detachment radius for electrolysis at various current densities. The measured radii are smaller than what equation (3.9) predicts, as one would expect from the contact line pinned somewhere inside the pit (position I) and a potential necking process [103]. Histograms of the detachment radii per current density applied to the same sample are included in Figure 3.8B. The detachment radius does not seem to be affected by the current density. The inset in Figure 3.8A likewise shows that the detachment radii of successive $\mathrm{CO}_{2}$ bubbles always fall below the theoretical value. Moreover, the measured radii slightly decrease with each successive bubble formed due to the onset of buoyancy-driven convection near the bubbles [113]. However, in general terms, bubble detachment radii remain stable and reproducible, especially in the short term (below 1 hour) [103].

Figure 3.9 shows two histograms of the detachment radii $R_{d}$ normalized with the theoretical maximum detachment radius $R_{d}^{*}$. The top histogram shows that the detachment radii of $\mathrm{CO}_{2}$ bubbles spread over a range with a mean value of $\sim 0.6 R_{d} / R_{d}^{*}$. The lower histogram shows a peaked distribution for the $\mathrm{H}_{2}$ bubble detachment radii, also with a mean value of $\sim 0.6 R_{d} / R_{d}^{*}$. The same mean range of $R_{d} / R_{d}^{*}$ for the $\mathrm{H}_{2}$ and $\mathrm{CO}_{2}$ measurements is not accidental since both scenarios make use of similar microstructures with the same pit-pillar configuration. The spread in the measured radii must arise from the fact that the contact line may differ from experiment to experiment, and thus the necking before pinch-off occurs 

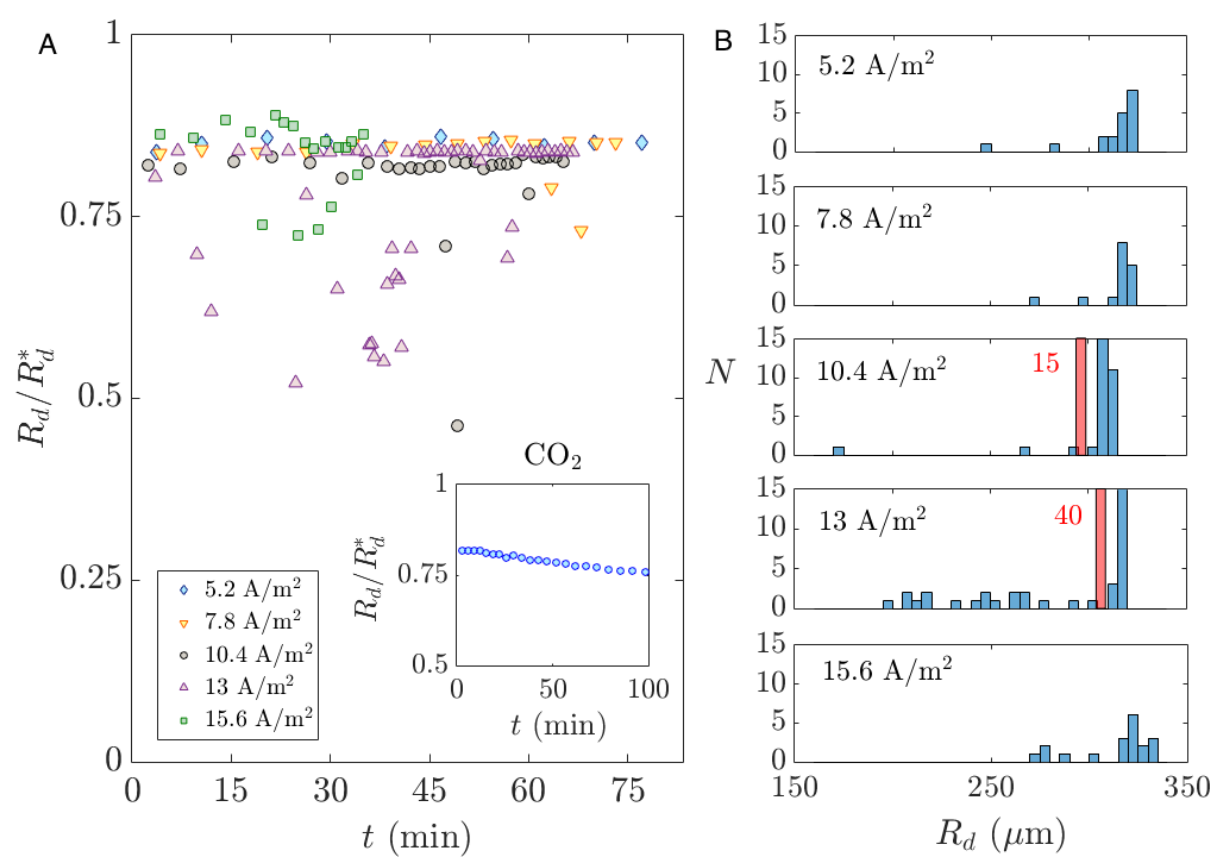

Figure 3.8: A) Successive $\mathrm{H}_{2}$ bubble detachment radii over time, normalized by the Fritz radius (3.9) for various levels of applied current density. The inset shows the normalized detachment radii of successive $\mathrm{CO}_{2}$ bubbles over time for $\zeta=0.17$. In this case, the detachment radii slightly decrease with time due to induced density-driven convection, which does not occur in electrolysis. B) Histograms of the measured detachment radius for various applied current densities (from top to bottom, $5.2 \mathrm{~A} / \mathrm{m}^{2}, 7.8 \mathrm{~A} / \mathrm{m}^{2}, 10.4$ $\mathrm{A} / \mathrm{m}^{2}, 13 \mathrm{~A} / \mathrm{m}^{2}$ and $15.6 \mathrm{~A} / \mathrm{m}^{2}$ ). The red bars at current densities 10.4 $\mathrm{A} / \mathrm{m}^{2}$ and $13 \mathrm{~A} / \mathrm{m}^{2}$ have values of $N=15$ and $N=40$, respectively. 
differently. Preferred adhesion sites or defects within the pit or on the pillar could be responsible for this. Since roughness of flat electrodes has been shown to influence the detachment radii of bubbles [165, 166, 167], we expect that pit roughness might play a role in the detachment radii of evolving bubbles. We measured the roughness in radial direction but found no apparent correlation between the detachment radii and the radial roughness (see the Electronic Supplementary Information). For some bubbles, $R_{d} / R_{d}^{*}>1$, probably due to the fact that the bubbles were not pinned to the pit (positions I or II in Figure 3.7A) but rather to defects on the pillar or the outer rim (position III, IV or V in Figure 3.7A). In our experiments, we have measured detachment radii up to $1.5 R_{d}^{*}$, especially for the case of the smallest pit to pillar radii ratio. This case is particularly interesting, since such a small ratio could be used for future designs of pillars in which the pit functions as the gas trapping source and the pillar as the outer pinning geometry for the bubble. Convective forces, electrostatic charges induced by local $\mathrm{pH}$ changes, and the dependency of surface tension and liquid density with concentration of dissolved gases may also influence the force balance and final detachment radius in a complex way. Although we provide several possible scenarios and parameters which could cause the deviation between the measured detachment radii and theory, the influence of other unknown factors can not be excluded. Nonetheless, a full analysis of the force balance and other factors influencing detachment is beyond the scope of this study.

\subsubsection{Gas transport efficiency}

The efficiency of gas transported away from the electrode surface by the bubbles can be quantified as the ratio between the amount of gas moles within each bubble after detaching from the nucleation site, $n_{b}$, and the total amount of electrolytically produced moles of $\mathrm{H}_{2}, n_{g}$. Note that this efficiency is not constant in time since it changes as the subsequent bubbles grow at different rates and depends on the amount of dissolved $\mathrm{O}_{2}$ which is reduced at the electrode. The efficiency after the $n$-th bubble in the succession has detached is thus calculated as

$$
\frac{n_{b}}{n_{g}}=\frac{\sum_{i=1}^{n} \frac{4 \pi P_{b, i} R_{d, i}^{3}}{3 R_{u} T}}{Q /(F z)}
$$

Here, $R_{d, i}$ denotes the detachment radius of the $i$-th bubble, $P_{b, i}=$ $\left(2 \sigma / R_{d, i}\right)+P_{l}$ is the internal pressure of the $i$-th bubble, $\mathrm{F}=96485 \mathrm{C} / \mathrm{mol}$, $z=2$ is the valency of the $\mathrm{H}_{2}$ evolution reaction, $Q\left(t_{n}\right)=J \pi R_{e}^{2} t_{n}$ is the total electric charge supplied at the detachment time of the $n$-th bubble $t_{n}$, $R_{u}=8.314 \mathrm{~J} / \mathrm{K} \cdot \mathrm{mol}$ is the universal gas constant and $T=293 \mathrm{~K}$ the absolute 


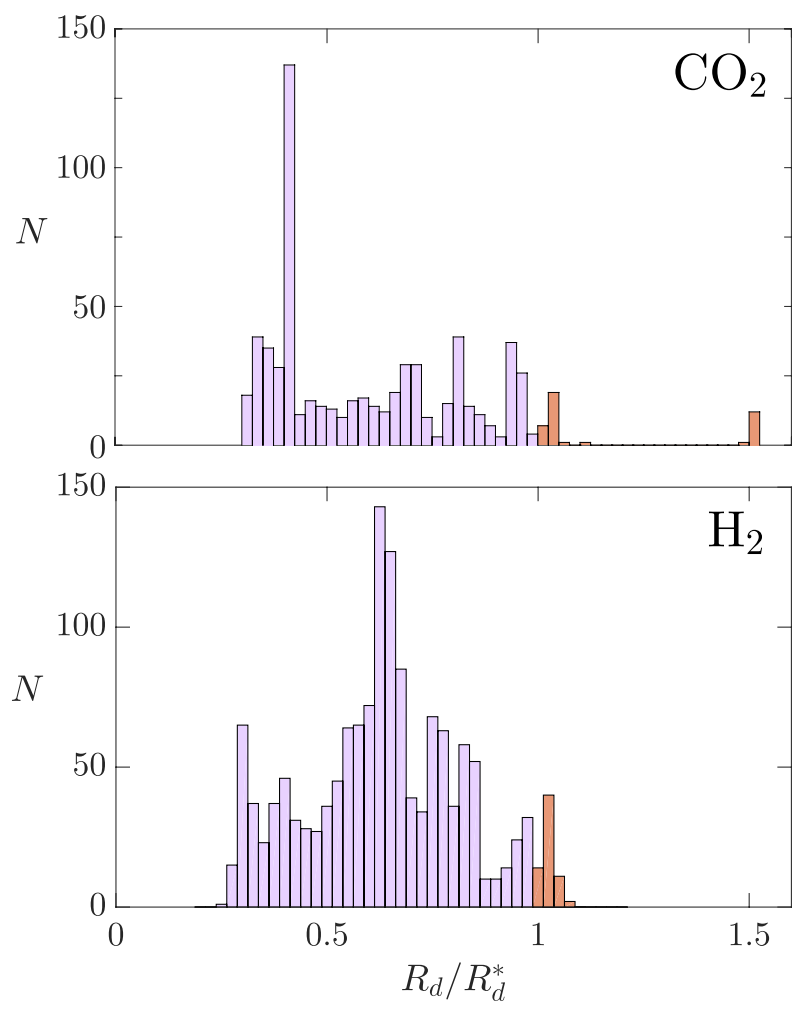

Figure 3.9: The top histogram shows the detachment radii of $\mathrm{CO}_{2}$ bubbles normalized by the Fritz radius (3.9) formed at $0.16<\zeta<0.18$. Values below $R_{d} / R_{d}^{*}<1$ are shown in purple whereas larger values are shown in red. The bottom histogram shows the detachment radii of $\mathrm{H}_{2}$ bubbles evolved on various substrates at different current densities. Here the same color palette as in the top figure has been used. Even though the histograms have different distributions, both correspond to a mean value of bubble detachment $R_{d} / R_{d}^{*} \approx 0.6$.

temperature. Note that this definition of the efficiency is limited to the gas transported away in each bubble and, therefore, does not consider the gas transport from the electrode in the form of convective plumes caused by bubble detachment or in the form of parasitic bubbles growing in other spots within the set-up.

Figure 3.10 shows the $\mathrm{H}_{2}$ transport efficiency of the bubble succession as a function of time. A single substrate is used for the measurements of the various current densities. The efficiency evolves as a parabola in time for all current densities, i.e. a similar trend as that of $\tilde{b}$ in time, Figure 
3.6. This originates from the definition of the transport efficiency, equation (3.10), which fundamentally corresponds to a discrete integral of $\tilde{b}$ in time. Consequently, the efficiency initially decreases due to the effect of depletion during the stagnation regime, region I in Figure 3.6. During the stagnation, the efficiency is surprisingly higher for lower current densities. This may originate from larger depletion losses caused, for instance, by the formation of parasitic bubbles. However, the efficiency becomes larger with increasing current densities as the concentration boundary layer evolves with time to higher gas concentrations. This is expected since the current density is directly proportional to the generation rate of molecular hydrogen. The produced gas does not diffuse fast enough into the bulk electrolyte, but accumulates instead around the bubble and electrode, increasing the local supersaturation. This results in faster bubble formation frequencies and higher transport rates. We find the highest experimental efficiency (5.7\%) for a current density of $7.8 \mathrm{~A} / \mathrm{m}^{2}$ after 270 minutes of constant electrolysis operation. A general optimal efficiency value could not be determined due to the eventual pit deactivation or parasitic bubble formation blocking optical access.

Future designs of electrodes with multiple nucleation sites may increase the amount of gas that is transported away by the bubbles, resulting in higher transport efficiencies. The size of the nucleation sites and the spacing over the surface would be crucial since they determine to what degree the bubbles compete for gas and how the gas concentration boundary layer evolves with time.

\subsection{Conclusions}

The microfabrication of artificial nucleation sites (in the form of pillar-pit microstructures on flat silicon substrates) allowed us to experimentally study bubbles evolving in water. By observing the succession of single bubbles, we compared the differences between the pressure-controlled supersaturated $\mathrm{CO}_{2}$ and electrolytic $\mathrm{H}_{2}$ bubbles, focusing on the evolution of the concentration boundary layer and its effect on the bubble growth rate, the detachment radius and the gas transport efficiency.

The time taken for the first $\mathrm{H}_{2}$ bubble to nucleate after the start of electrolysis at various current densities coincides with previous electrolysis nucleation studies and covers a wide spread ranging from the order of seconds to tens of seconds (most probably affected by the presence of dissolved $\mathrm{O}_{2}$ at the beginning of the experiment) whereas the $\mathrm{CO}_{2}$ nucleation occurs generally in the order of seconds once the carbonated solution becomes supersaturated. By studying the growth coefficient $\tilde{b}$, we determine that a system with a finite amount of gas available will 


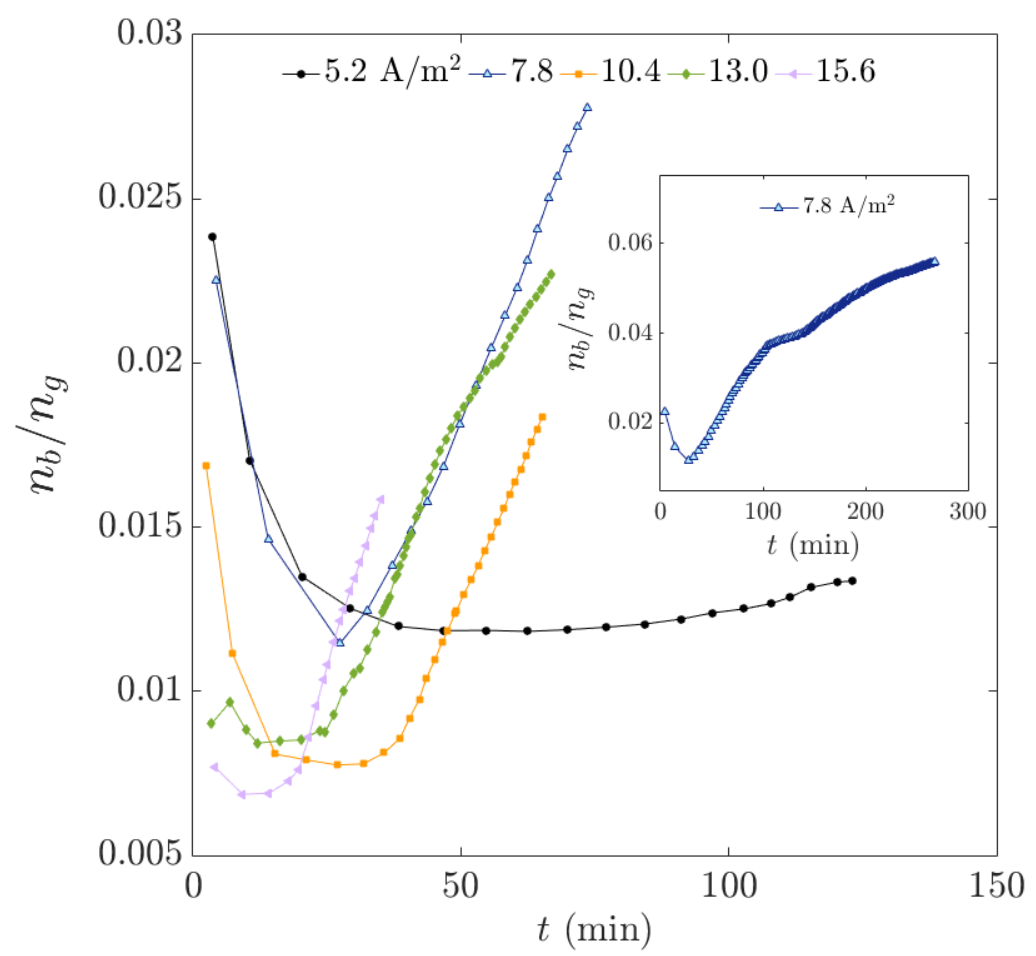

Figure 3.10: The ratio of gas transported out of the liquid phase by the bubbles and the amount of electrolytically generated gas as function of time for various current densities. For the measurement at $5.2 \mathrm{~A} / \mathrm{m}^{2}$, the current density is so low that only regime I appears within our experimental time. It is expected that the other regimes (II \& III) would occur with prolonged reaction time. The inset shows the efficiency ratio for the full length of the $7.8 \mathrm{~A} / \mathrm{m}^{2}$ measurement up to 270 minutes. A maximum efficiency of $5.7 \%$ is obtained at the end of the experiment. The employed nucleation site has a radius of $R_{0}=5 \mu \mathrm{m}$.

experience continuously slower bubble evolution over time due to gas depletion, whereas in the case of electrolytically generated bubbles, their growth experiences different phases depending on the concentration of available gas as a function of time. The height of the pillars does not seem to play any significant role during bubble evolution in any of the cases studied here.

Bubble detachment usually occurs around $60 \%$ of the maximum theoretical radius (see equation (3.9)) for both cases. This fact indicates that bubble detachment is mainly governed by the pillar-pit geometry. The smaller detachment value originates from the structural imperfections of 
the pits that lead to random adhesion sites of the contact line. The contact angle, the force balance and the neck formation of the bubbles are thus affected. For $\mathrm{CO}_{2}$ bubbles, detachment occurs at slightly decreasing radii over time because of the onset of density driven convection [113] and a neck formation between the trapped gas in the pit and the growing bubble on top [103]. In electrolysis, the detachment of $\mathrm{H}_{2}$ bubbles does not follow any clear trend.

Finally, the gas evolution efficiency follows a parabolic trend with time. A matching trend is observed for the bubble growth rates. We conclude that the efficiency first decreases due to depletion losses, and then increases after a certain supersaturation is achieved and the dissolved $\mathrm{O}_{2}$ is reduced. Surprisingly, during the stagnation regime the efficiency is higher for lower current densities. This effect is counteracted later in time, such that higher current densities $J$ imply higher efficiencies. The maximum efficiencies range from 1 to $5 \%$, values which could be further increased with the use of multiple nucleation sites and flow conditions, closer to real life applications where continuous flow reactors are desirable. The aspects of nucleation, growth, and detachment considered here certainly warrant future studies toward higher transport efficiencies of (photo)electrolytic devices.

\section{Acknowledgements}

We would like to thank S. Schlautmann for the discussion and fabrication of the experimental substrates, and R. P. G. Sanders for the discussions regarding the electrolysis set-up. We would further like to extend our thanks to H. A. G. M van Wolferen for the FIB milling and SEM imaging and the MESA+ Nanolab for the use of their facilities. This work was supported by the Netherlands Center for Multiscale Catalytic Energy Conversion (MCEC), an NWO Gravitation programme funded by the Ministry of Education, Culture and Science of the government of the Netherlands. 


\section{Appendix A: Supplementary information}

\section{Roughness study and its effect on the bubble detachment radius}

The roughness in the radial direction at the top of the pits is measured by imaging the apex from the top using Scanning Electron Spectroscopy (SEM). A circle-line is fitted over the pit apex and deviations from the ideal circumference are measured. We then calculate the roughness in radial direction as:

$$
R_{a}=\frac{1}{n} \sum_{i=1}^{n}\left|y_{i}\right|,
$$

where $y_{i}$ is the deviation per measurement point. For these calculations, we take points with equal spacing over each circle. We calculate $R_{a}$ for various DRIE conditions and fluorocarbon deposition times, but a correlation to the detachment radius could not be found.

To illustrate the complexity of taking the surface roughness into account, we image various substrates. Figure $3.11 \mathrm{~A}$ shows partially the inside of a pit with a $7.5 \mu \mathrm{m}$ radius. Here the black silicon is visible at the bottom half of the image, the top half shows part of the inner wall of the $20 \mu \mathrm{m}$ deep pit where fluorocarbons have been deposited for 37 seconds after the pit formation. The wall shows non-homogeneity in the surface created during the pit fabrication, which could play an important factor if the bubble is pinned within the pit. A top view of a pillar with a $15 \mu \mathrm{m}$ radius is shown in Figure 3.11B, which contains a defect on the outer edge at the bottom right side, providing an irregularity that could influence the bubble detachment size if the contact line moves to the pillar edge. Figure 3.11C shows a close-up of the pit in the pillar of Subfigure $\mathrm{B}$, in which the pit apex is shown in bright white. The pit has a radius of $7.5 \mu \mathrm{m}, R_{a}=0.004 \mu \mathrm{m}$ averaged by three measurements, and due to the substrate not being levelled under the electron source, a small part of the wall is shown at the right side of the image between the dark black silicon and the bright pit apex. Figure 3.11D shows a top view of a $30 \mu \mathrm{m}$ tall micropillar, with a radius of $15 \mu \mathrm{m}$ and pit radius of $5 \mu \mathrm{m}$. Before imaging, this substrate was used in an electrolysis experiment, during which an unknown substance precipitated on the pillar surface near the pit, creating additional pinning locations for bubbles. Subfigure E shows a close-up of this pit with $R_{a}=0.008 \mu \mathrm{m}$ averaged by three measurements. Similar to Subfigure C, a part of the pit wall is shown on the right side between the dark black silicon and bright apex. Although in our experiments roughness does not seem to play any significant role during bubble growth and detachment, its influence warrants further investigation which does 
not only take into account the pit apex but the roughness of the pit inner wall, the pillar outer wall and the top pillar surface, as well as other defects.

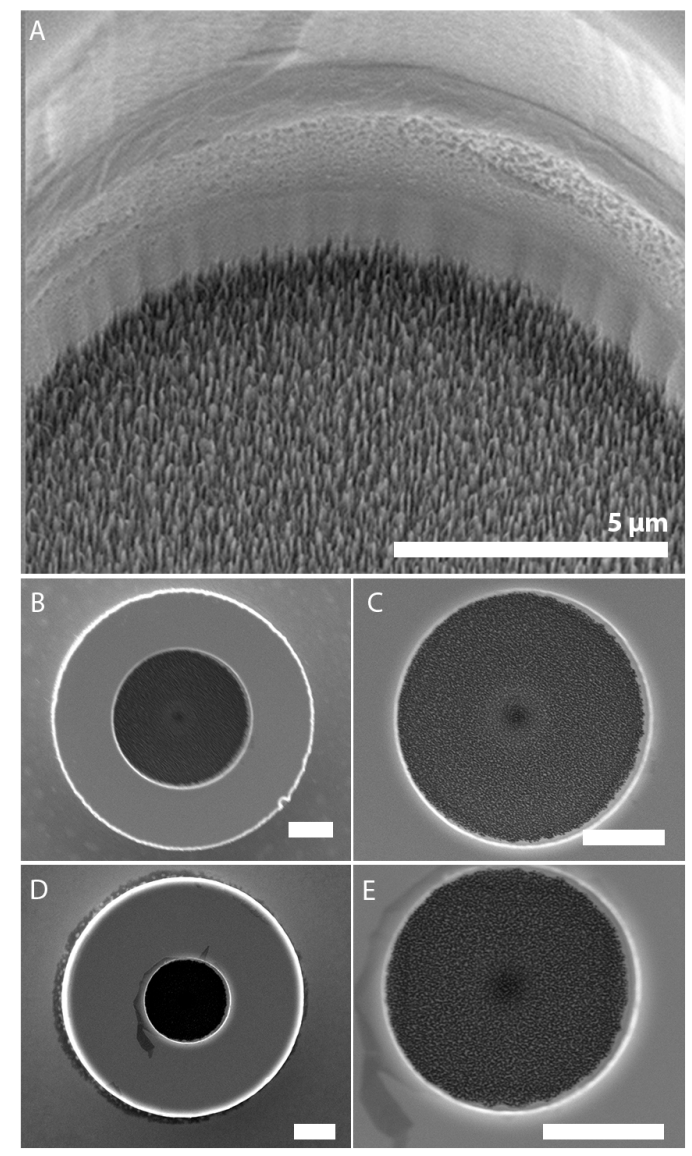

Figure 3.11: A) The inside of a micropit of $20 \mu \mathrm{m}$ depth and $7.5 \mu \mathrm{m}$ radius is partially shown. The substrate is under a $20^{\circ}$ angle with respect to the electron beam source. At the bottom of the image, black silicon is visible. At the top, the wall on the inside of the pit shows roughness, a result of the DRIE process. In all subfigures, the white scalebars indicate a $5 \mu \mathrm{m}$ length. B) A top view of a pillar with a $15 \mu \mathrm{m}$ radius and $7.5 \mu \mathrm{m}$ radius pit is shown. A defect from the fabrication of the substrate on the outer edge at the bottom right side of the pillar is present. C) Close-up of the pit shown in B. Here the radial roughness is $R_{a}=0.004 \mu \mathrm{m}$. D) A micropillar of 30 $\mu \mathrm{m}$ height, $15 \mu \mathrm{m}$ radius and a pit radius of $5 \mu \mathrm{m}$ is shown. A deposit on the surface of the pillar can be noticed near the pit aperture. E) Close-up of the pit from Subfigure D. Here, $R_{a}=0.008 \mu \mathrm{m}$. 


\section{Appendix B: Electronic supplementary information}

The following QR codes can be scanned to access the electronic supplementary information.

The QR code in Figure 3.12 leads to the first electronic supplementary information file. The file is a video of a micropillar being milled with a focussed ion beam to show the black silicon and fluorocarbon layer inside the superhydrophobic cavity. The following url will lead to this video as well: http://www.rsc.org/suppdata/c8/ee/c8ee02657b/c8ee02657b2. mp4.

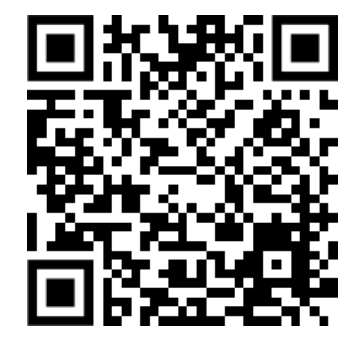

Figure 3.12: Roughness study and its effect on the bubble detachment radius.

The QR code in Figure 3.13 leads to the second electronic supplementary information file. The file is a video of bubble evolution at different current densities. The following url will lead to this video as well: http://www.rsc. org/suppdata/c8/ee/c8ee02657b/c8ee02657b3.mp4.

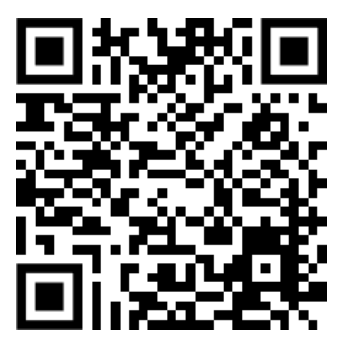

Figure 3.13: Bubble evolution at different current densities. 


\section{Electrolysis-driven and pressure-controlled diffusive growth of successive bubbles on micro-structured surfaces}

Control over the bubble growth rates forming on the electrodes of water-splitting cells or chemical reactors is critical towards the attainment of higher energy efficiencies within these devices. This study focuses on the diffusion-driven growth dynamics of a succession of $\mathrm{H}_{2}$ bubbles generated at a flat silicon electrode substrate. Controlled nucleation is achieved by means of a single nucleation site consisting of a hydrophobic micropit etched within a micron-sized pillar. In our experimental configuration of constant-current electrolysis, we identify gas depletion from (i) previous bubbles in the succession, (ii) unwanted bubbles forming on the sidewalls and (iii) the mere presence of the circular cavity where the electrode is being held. The impact of these effects on bubble growth is discussed with support from numerical simulations. The time evolution of the dimensionless bubble growth coefficient - a measure of the overall growth rate of a particular bubble - of electrolysis-generated bubbles is compared with that of $\mathrm{CO}_{2}$ bubbles growing on a similar surface in the presence of a supersaturated solution of carbonated water. For electrolytic bubbles, and under the range of current densities considered here $\left(5-15 \mathrm{~A} / \mathrm{m}^{2}\right)$, it is observed that $\mathrm{H}_{2}$ bubble successions at large gas-evolving substrates first experience a stagnation regime, followed by a fast increase in the growth coefficient before a steady state is reached. This clearly contradicts the common assumption that constant current densities must yield time-invariant growth rates. Conversely, for the case of $\mathrm{CO}_{2}$ bubbles, the growth coefficient successively decreases for every subsequent bubble due to the persistent depletion of dissolved $\mathrm{CO}_{2} \cdot{ }^{1}$

\footnotetext{
${ }^{1}$ Reprinted with permission from (van der Linde, P. Moreno Soto, Á. Peñas-López, P. Rodríquez-Rodríquez, J. Lohse, D. Gardeniers, H. van der Meer, D. and Fernández Rivas, D. Electrolysis-Driven and Pressure-Controlled Diffusive Growth of Successive Bubbles on Microstructured Surfaces, Langmuir, 2017, 33(45), pp 12873-12886. DOI: 10.1021/acs.langmuir.7b02978). Copyright (2019) American Chemical Society.
} 


\subsection{Introduction}

At sufficiently large voltages, gas bubbles are produced on the electrodes in electrochemical reactors [168, 169, 46], water-splitting cells during the electrolysis of water for hydrogen production [56] or the study of nanobubbles [170, 160, 171], and during photoelectrolysis by solar-driven cells $[172,58]$, a topic which recently has gained interest. The formation of unwanted nucleation sites or large bubble departure sizes may lead to an excessive coverage of electrodes by bubbles. This ultimately results in increased electrical resistances [70, 56] within the aforementioned devices. Thus, proper control over the nucleation, growth and detachment of bubbles is expected to play a key role in the progress towards higher energy efficiencies [28]. Moreover, such control should allow to efficiently harvest the produced gas bubbles [98] without further need of energy input, e.g. in the form of pumping- or vibration-based mechanical systems.

Control over the bubble growth rates is a critical aspect that should be achieved after proper understanding of the underlying physical problem. Bubble growth kinetics in electrolytic systems have been generally described in the form of $[173,174,92,97,93,175,176,23,100]$

$$
R=b t^{\alpha},
$$

where $R$ is the bubble radius, $t$ is the actual residence time of the bubble - which starts growing at $t=0-$ on the electrode before detachment. Here, $b$ is the dimensional growth coefficient whereas $\alpha$ is the scaling exponent. Throughout the majority of the bubble lifetime, except for a very fast inertia-controlled initial growth [92] (of the order of $0.1 \mathrm{~s}$ in our experiments), $b$ and $\alpha$ can be approximated as constants. The scaling exponent $\alpha$ is typically $1 / 2$ or $1 / 3$, depending on the experimental conditions.

The occurrence of one or the other scaling exponent is determined by a quantity of immediate interest, $A_{e} / R_{d}^{2}$, namely the ratio of the active surface area, $A_{e}$, of the electrode upon which the bubble grows to the characteristic bubble surface at the moment of detachment (at a bubble radius $R_{d}$ ). This coefficient represents the ratio between the characteristic time of the diffusive transport of the evolved gas across a region of the size of the electrode, $t_{t} \sim A_{e} / D$, and that of the diffusive transport of gas to the bubble, namely $t_{d} \sim R_{d}^{2} / D$, which in turn limits the bubble growth rate. Here, $D$ denotes the gas diffusivity in the liquid. Alternatively, if the exsolution of dissolved gas into the bubble is considered as a chemical reaction, the quantity $A_{e} / R_{d}^{2}$ can be interpreted as a Damköhler number representing the ratio between the diffusive transport time to the bulk fluid and the diffusive transport time of gas into the bubble. This ratio is 
geometrically imposed by the experimental configuration and it essentially specifies the growth mechanism which determines the nature of $b$ and $\alpha$ [100]. Two limiting cases $[174,100]$ are to be identified:

(i) Surface-reaction-controlled (or production/reaction-limited) growth for relatively small active electrode surfaces, $A_{e} / R_{d}^{2} \ll 1$, where $R=$ $b t^{1 / 3}$.

(ii) Diffusion-controlled (or diffusion-limited) growth for relatively large active electrode surfaces: $A_{e} / R_{d}^{2} \gg 1$, where $R=b t^{1 / 2}$.

Bubbles generated at microelectrodes [92, 93, 23] by catalysis, and at laser-irradiated photoelectrodes $[177,100]$ or plasmonic nanoparticles [178] are common scenarios of case (i). Here, bubble growth is directly limited by the gas production rate and hence by the supplied current. Essentially, all the gas produced at the surface of the electrode directly diffuses into the bubble adhered to it. Gas diffusion into the bulk electrolyte is negligible in comparison. The gas evolution efficiency [179], namely the fraction of gas contained within the departing bubble(s) to the total amount of gas produced at the electrode, has been reported to be close to $100 \%$ [23]. For these reasons, microelectrodes enable the production of well-controlled successions of single bubbles that nucleate, grow and detach in a highly periodic and repetitive fashion. In the case of a single microelectrode, multiple bubble interactions or bubble coalescence events are suppressed. Moreover, the effect of convection induced by the detachment of previous bubbles on the growth rate is negligible since viscous dissipation limits detachment-induced flow to a short time interval of around $0.1 \mathrm{~s}$ [23]. The mathematical formulation of the growth dynamics in case (i) is trivial. Provided that the growing bubble remains spherical as it grows and that the contact angle is small, a simple mass balance yields the following experimentally-confirmed relation [96, 92, 93, 23]:

$$
R=b t^{1 / 3}=\frac{3 R_{u} T_{\infty} I}{4 \pi F v P_{0}} t^{1 / 3},
$$

where $R_{u}$ is the universal gas constant, $T_{\infty}$ and $P_{0}$ are the liquid pressure and temperature, $I$ is the electric current, $F$ is Faraday's constant and $v$ is number of electrons transferred in the electrochemical cell, per molecule of evolved gas.

The mechanism behind case (ii) - the case of bubbles growing on electrodes with relatively large surfaces - is substantially different. Growth is almost entirely driven by Fickian diffusion of gas into the bubble from the surrounding supersaturated electrolyte. Mass is now transferred across the entire bubble surface. Direct gas diffusion from the substrate to the 
bubble is, on the other hand, usually small in comparison. We then expect a very similar behavior to the diffusive, quasi-static growth of bubbles in uniformly supersaturated solutions [166, 113]. Diffusion-limited growth has been found to be well described by the classical theories by Epstein \& Plesset [14] or Scriven [101]. These theories - which can be extended to the growth of surface (nano)bubbles [180] - coincide in that diffusive growth may be described by $R=b t^{1 / 2}$. The scaling $R \propto t^{1 / 2}$ is in full agreement with reported electrolysis experiments for case (ii) [173, 97, 175, 176].

The diffusive growth coefficient $b$ is now determined by the local degree of supersaturation of the nearby liquid, by the gas solubility and the gas diffusivity. The local degree of supersaturation and, in consequence, the average bubble growth rate and gas evolution efficiency are all expected to increase with the current density [179]. Most studies reporting bubble growth dynamics $[173,174,97,100]$ or analysing mass transfer from electrodes [181, 179, 182] assume a constant, time-independent value of the local supersaturation for a given current density. This therefore would entail a unique growth coefficient $b$ common to all bubbles, regardless of the time of nucleation after the onset of electrolysis. However, it follows that the gas supersaturation near the electrode not only depends on the current density, but also on the elapsed time of electrolysis. This stands to reason, since, from simple mathematical considerations [175], the imposition of a constant gas flux at the electrode surface must lead to an accumulation over time of dissolved gas near the electrode. The concentration of dissolved gas increases with time until an approximately steady-state profile is reached. Consequently, $b$ is expected to increase for subsequent bubbles until a steady-state value is reached, even under the imposition of a constant current density.

The constant-electrolysis scenario bears some similarities with the diffusion-driven growth of a succession of bubbles from a nucleation site in a supersaturated liquid. In both scenarios, the behavior $R=b t^{1 / 2}$ holds for each individual bubble, and the value of the coefficient $b$ is observed to discretely vary from bubble to bubble in the succession. However, in the latter scenario the growth coefficient $b$ of subsequent bubbles continuously gets smaller and smaller, as opposed to becoming increasingly larger as seen during electrolysis. This effect has been recently studied for a succession of $\mathrm{CO}_{2}$ bubbles in carbonated water kept at constant pressure under supersaturation conditions [103].

With all these ideas in mind, the aim of this work is to explore in detail the time evolution of the growth coefficient for a succession of $\mathrm{H}_{2}$ bubbles produced by constant-current electrolysis. In our configuration, the electrode is much larger than the bubble, hence $R=b t^{1 / 2}$ is expected. We make use of flat silicon electrodes with a single microstructure upon 
which a succession of single bubbles may form. This way, the effect on $b$ of bubble electrode coverage, convection and diffusive interactions between multiple bubbles [182] is intentionally minimised. This is done in view of future applications in photoelectrolysis, where silicon is becoming an increasingly popular material for photoelectrodes [183, 184], considering that it may be feasibly etched with micrometre- or nanometre-sized pillars [154] as a means to increase light-harvesting efficiencies and gas production rates. Finally, some interesting conclusions will be drawn by comparing the growth coefficient of electrolysis bubbles with that of $\mathrm{CO}_{2}$ bubbles in carbonated water in a pressure-controlled (P-C) set-up.

\subsection{Experiments}

\subsubsection{Micro-structured substrates}

A single active site for bubble formation was defined at the centre of flat boron-doped silicon substrates $(10 \times 10 \times 0.525 \mathrm{~mm})$. This nucleation site, fabricated through reactive ion etching after photolitography, consists of a micron-sized hydrophobic cylindrical pit [150] (about $20 \mu \mathrm{m}$ deep) etched within a cylindrical micron-sized pillar [154]. Details of the fabrication process may be found elsewhere [185].

Figure 4.1 shows a scanning electron microscope image of a micropillar containing the superhydrophobic pit. The pit radius, $R_{0}$, was set in the range of $1-10 \mu \mathrm{m}$ over the various experimental samples, values which ensure successful bubble nucleation [156]. Similarly, the pillar radius, $R_{p}$, was set to 5-15 $\mu \mathrm{m}$. The pillar heights considered were $H_{p}=0,15$ and 30 $\mu \mathrm{m}$, in view of potentially identifying any influence that the distance from the electrode to the bubble nucleation spot may have on bubble growth. The case $H_{p}=0 \mu \mathrm{m}$ corresponds to a micropit in an otherwise flat substrate, i.e. the pillar is absent in this case.

\subsubsection{Experimental method}

The experimental set-up, sketched in Figure 4.2, comprises a 3D-printed acrylic holder and a mounting plate designed to hold the silicon substrate (electrode) in place. The substrate is at the base of a circular cavity (of radius $R_{e}=3.5 \mathrm{~mm}$ and depth $H_{c}=2 \mathrm{~mm}$ ) that determines the active electrode surface in contact with the electrolyte. A rubber ring, placed between the silicon substrate and the substrate holder, creates a liquid-tight seal. A counter electrode in the form of a platinum wire is then placed at the corner of the holder far away from the substrate.

At the beginning of each experiment, the holder is filled with $20 \mathrm{~mL}$ of 


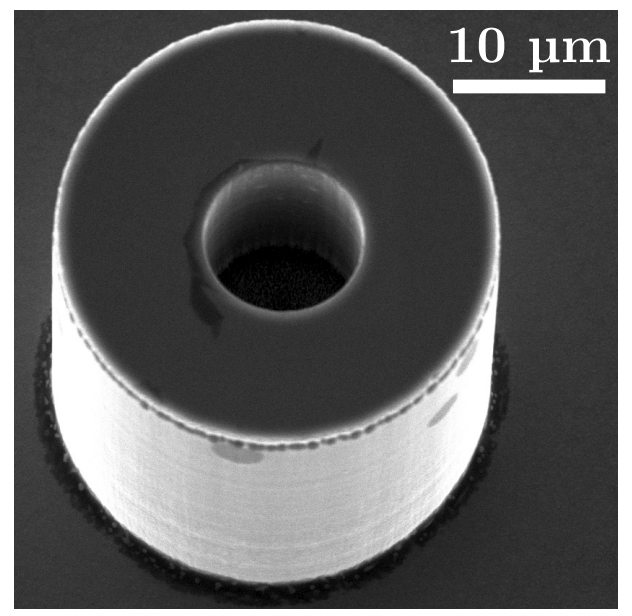

Figure 4.1: SEM image of a micropillar protruding from the silicon substrate. In this particular sample, the pillar height is $H_{p}=30 \mu \mathrm{m}$, the pit (inner) radius is $R_{0}=5 \mu \mathrm{m}$ and the pillar (outer) radius is $R_{p}=15 \mu \mathrm{m}$.

fresh electrolyte. The resulting electrolyte level depth is $H \approx 12 \mathrm{~mm}$. The electrolyte consists of a solution composed of non-degassed milli-Q water with $10 \mathrm{mM}$ of $\mathrm{Na}_{2} \mathrm{SO}_{4}$ salt and a pH 3 buffer (sodium acetate anhydrous and acetic acid), giving the bubbles a neutral electric charge [92]. During each experiment, a constant current $I$ in the range of $200-600 \mu \mathrm{A}$ is supplied. The corresponding current density, computed as $I_{w}=I /\left(\pi R_{e}^{2}\right)$, falls in the range of $5-15 \mathrm{~A} / \mathrm{m}^{2}$. This way, a constant molar flux of hydrogen gas, $J_{w}=I_{w} /(F v)$, is uniformly released from the substrate by electrolysis. Here, $F=96485 \mathrm{C} / \mathrm{mol}$ is Faraday's constant and $v=2$ is the number of electrons taken up per molecule of evolved $\mathrm{H}_{2}$ gas.

A few seconds after the start of electrolysis, the electrolyte is sufficiently saturated with hydrogen and a single bubble grows from the pit on top of the micropillar. Eventually, the bubble reaches a critical size and detaches. Shortly afterwards a new bubble nucleates and the process starts over again. Images of the succession of growing bubbles are acquired from the top by a digital camera mounted with an axial light-source during one to up to five hours.

The radius of the gas-evolving substrate is about 10 times larger than the typical detachment radius of the bubble $\left(R_{d}<0.4 \mathrm{~mm}\right)$, which gives a Damköhler number $D_{e}=R_{e}^{2} / R_{d}^{2} \gtrsim 80 \gg 1$. As discussed in the introduction, it is expected that the bubble dynamics then follows diffusion-limited growth. 


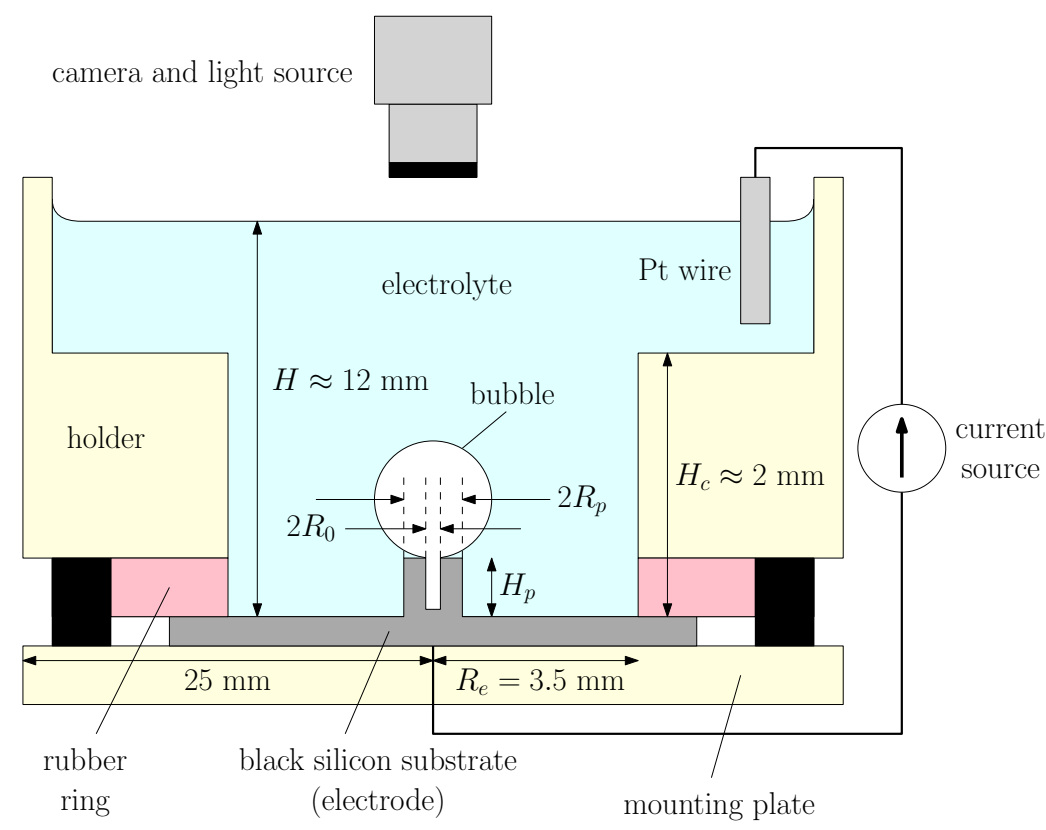

Figure 4.2: Sketch of the electrolysis set-up. The dimensions of the substrate, cavity and holder are not drawn to scale to highlight, within a single schematic, the different dimensions playing a crucial role in electrolytic bubble growth. Note that the pit and pillars are much smaller in reality.

\subsubsection{Experimental observations: electrolysis-induced bubbles}

Figure 4.3 shows the typical evolution of the bubble radius for a succession of bubbles captured during the first hour of constant $I_{w}$. Several effects come into view. Firstly, the bubble growth rates eventually increase with time. This stands to reason since imposing a constant flux $J_{w}$ leads to an accumulation of dissolved $\mathrm{H}_{2}$ gas in the bubble surroundings. In other words, there is a net increase of the average $\mathrm{H}_{2}$ concentration in the region where the bubble grows. The fact that a repetitive, steady-state growth is not reached immediately is very striking and significant. The time required to reach the steady state in the absence of bubbles (see Appendix A) can be very roughly estimated as $t_{s s} \approx H^{2} / D_{H_{2}}$, where $D_{H_{2}}=4.2 \times 10^{-9} \mathrm{~m}^{2} / \mathrm{s}$ is the diffusivity of $\mathrm{H}_{2}$ in the electrolyte [186]. To do so, we have assumed a 1D concentration profile $C(z, t)$ with a constant flux at $z=0$ together with a zero-concentration boundary condition at the free surface, $C(H, t)=0$. Taking $H=10 \mathrm{~mm}$, this gives approximately 6 hours. In our experiments, it will be seen that the steady state is attained somewhat faster, within the order of $0.5-3$ hours. 
(a)

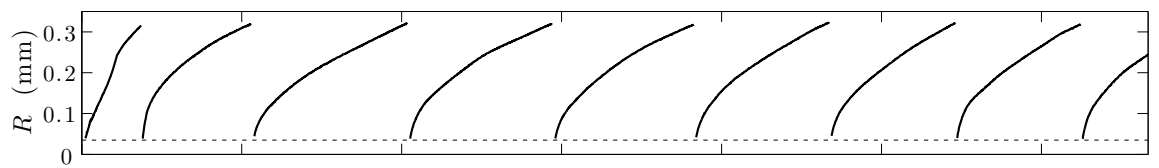

(b)

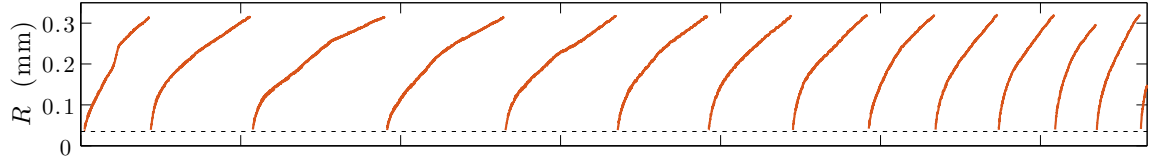

(c)

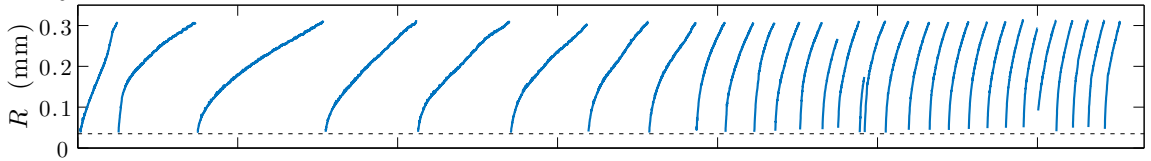

$(d)$

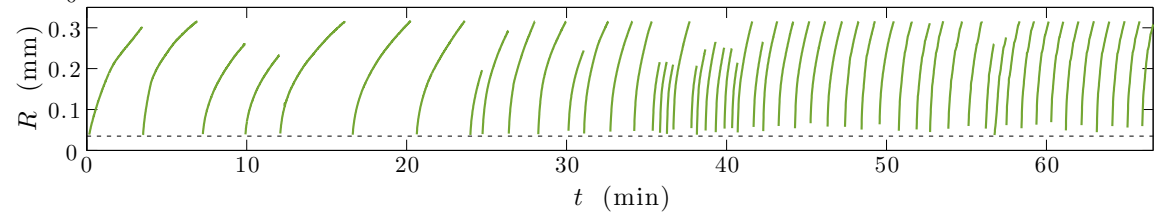

Figure 4.3: Time evolution of the bubble radius for a succession of bubbles captured during the first hour after the start of the electrolysis. Each individual curve represents a distinct bubble. Four different experiments are shown, in which the supplied current density is set to $(a) 5.2,(b) 7.8$, (c) 10.4 and $(d) 13.0 \mathrm{~A} / \mathrm{m}^{2}$. The pit and pillar radius are 5 and $15 \mu \mathrm{m}$, respectively, whereas the pillar height is $30 \mu \mathrm{m}$. Using $R_{d} \approx 0.3 \mathrm{~mm}$ and $R_{e}=3.5 \mathrm{~mm}$ yields a Damköhler number of $D_{e}=R_{e}^{2} / R_{d}^{2} \approx 136$. The dotted horizontal line marks the etching defect size below which it was not possible to optically detect the bubbles.

Secondly, the radius dynamics are affected by transient effects. This is especially evident in the very first bubble(s) of Figures 4.3(a)-(d), whose growth dynamics displays fast temporal variations. This is attributed to the unsteadiness and non-uniformity of the evolving concentration field, which makes it more susceptible to perturbations during the first minutes after the onset of electrolysis. In time, these transient effects diminish as the boundary layer of evolved $\mathrm{H}_{2}$ gas grows and the local concentration field surrounding the pillar becomes more uniform. The effect of the contact angle dynamics on bubble growth is discarded since the detachment radius is always found to be below the maximum theoretical Fritz radius [114] calculated using the pit circumference as the reference contact line. This implies that bubbles do not adhere to the top surface of the pillar. Instead, they are attached to the inside of the pit and detach through a necking process [103]. 


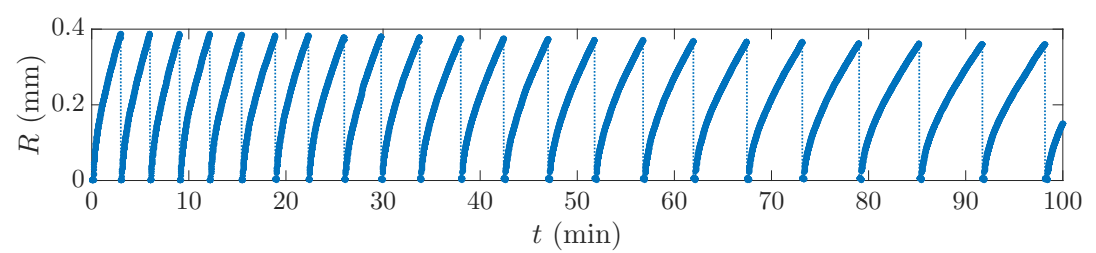

Figure 4.4: Pressure-controlled growth for a succession of $\mathrm{CO}_{2}$ bubbles for $\zeta=0.17$ during the first 100 minutes after the pressure drop. The dotted vertical lines indicate bubble detachment and nucleation of the following bubble. The pit and pillar radius are 10 and $15 \mu \mathrm{m}$, respectively, whereas the pillar height is $30 \mu \mathrm{m}$.

Thirdly, electrolytically driven bubble evolution is not perfectly reproducible for a number of reasons that will be discussed later. Premature detachment of particular bubbles is a commonly observed phenomenon in our experiments, especially for large current densities or supersaturations, as seen in Figure 4.3(d) for $t=24$ and $35 \mathrm{~min}$.

\subsubsection{Experimental observations: pressure-controlled bubbles}

It is of interest to see how the growth dynamics obtained in the electrolysis experiments compares with those found in pressure-controlled (P-C) experiments entailing a succession of $\mathrm{CO}_{2}$ bubbles produced in uniformly supersaturated water. To do so, a silicon chip, identical to those used in electrolysis experiments, is placed at the center of a pressurized test chamber (pressure $P_{s} \approx 9$ bars) that is then filled with carbonated water previously saturated under the same pressure. A detailed description of the experimental set-up and procedure may be found in the works by Enríquez et al. (2013)[153] and Moreno Soto et al. (2017)[103]. to which the reader is referred to. At the beginning of the experiment, the pressure is lowered down to a value $P_{0} \approx 8$ bars, which yields a supersaturation $\zeta=P_{s} / P_{0}-1 \approx 0.17$. Shortly afterwards, a single bubble nucleates and grows on top of the micropillar. As in the case of the electrolysis experiments, a succession of bubbles then follows. The time evolution of the bubble radii for a particular experiment is shown in Figure 4.4.

Two differences become immediately apparent upon comparison of Figures 4.3 and 4.4. In the first place, the P-C growth rate and detachment radius are more repeatable and stable than for the electrolysis experiments. In the second place, in the P-C experiments, the time taken for each bubble to detach grows with time, thus the growth coefficient decreases. This is in contrast to what is observed in electrolysis, where the continuous dissolution of $\mathrm{H}_{2}$ in the bubble surroundings enhances the growth rate. In 
the case of P-C bubble growth, Moreno Soto et al. (2017)[103] showed that the slowing down of the bubble growth is caused by the local depletion of $\mathrm{CO}_{2}$ near the bubble. Such a depletion phenomenon was attributed to buoyancy-driven convection and mixing due to bubble detachment.

\subsubsection{Numerical model}

As a means to provide further insight into the growth dynamics of the electrolytic bubbles studied here, a numerical model for the growth of a spherical bubble attached to a micropillar of height $H_{p}$ protruding from a (gas-evolving) substrate has been developed. It is an extension of the (pillar-less) numerical model described in Peñas-López et al. (2017)[187].

The governing equations are solved through a second order finite-difference discretisation in space and an implicit Euler method in time. The 2D axisymmetric grid is portrayed in Figure 4.18. The grid is divided into two regions that use different coordinate systems: a bulk region for $z>H_{p}$ and a microlayer region below. In the bulk region, the advection-diffusion equation coupled with the streamfunction-vorticity equations [187] are solved, imposing a no-slip boundary condition at $z=H_{p}$. In the microlayer region, only the diffusion equation is solved. The concentration and its gradient are matched at the coupling plane $z=H_{p}$. If required, a constant and uniform molar flux can be imposed at the substrate wall $(z=0)$ and pillar surface. Details about the simulation equations may be found in the supporting material.

The limitations of the simulation regarding the electrolysis experiments are that it neither considers the real finite size of the electrode, nor the presence of a cavity (see Figure 4.2). Moreover, we assume a spatially-uniform flux along the electrode. Finally, we must estimate the initial $\mathrm{H}_{2}$ field corresponding to a bubble nucleating at some time after the start of electrolysis. To do so, we use the 1D analytical concentration profile in the absence of bubbles provided in Appendix A. Thus, this idealised initial concentration profile just depends on two input parameters, namely, the effective current density (which establishes the flux of dissolved $\mathrm{H}_{2}$ gas that is evolved at the electrode) and the elapsed time of electrolysis. We must finally remark that, when modelling the growth of electrolytic bubbles, a binary system of $\mathrm{H}_{2}$ gas and air is considered. The electrolyte is not degassed; in fact it was kept in a container openly exposed to ambient air. Hence, we make the approximation that the electrolyte surrounding the micropillar is perfectly equilibrated with air at the beginning of bubble growth. The presence of dissolved air explains why the first hydrogen bubbles nucleate and grow after just a few seconds after the start of electrolysis when the electrolyte close to the electrode is still undersaturated 


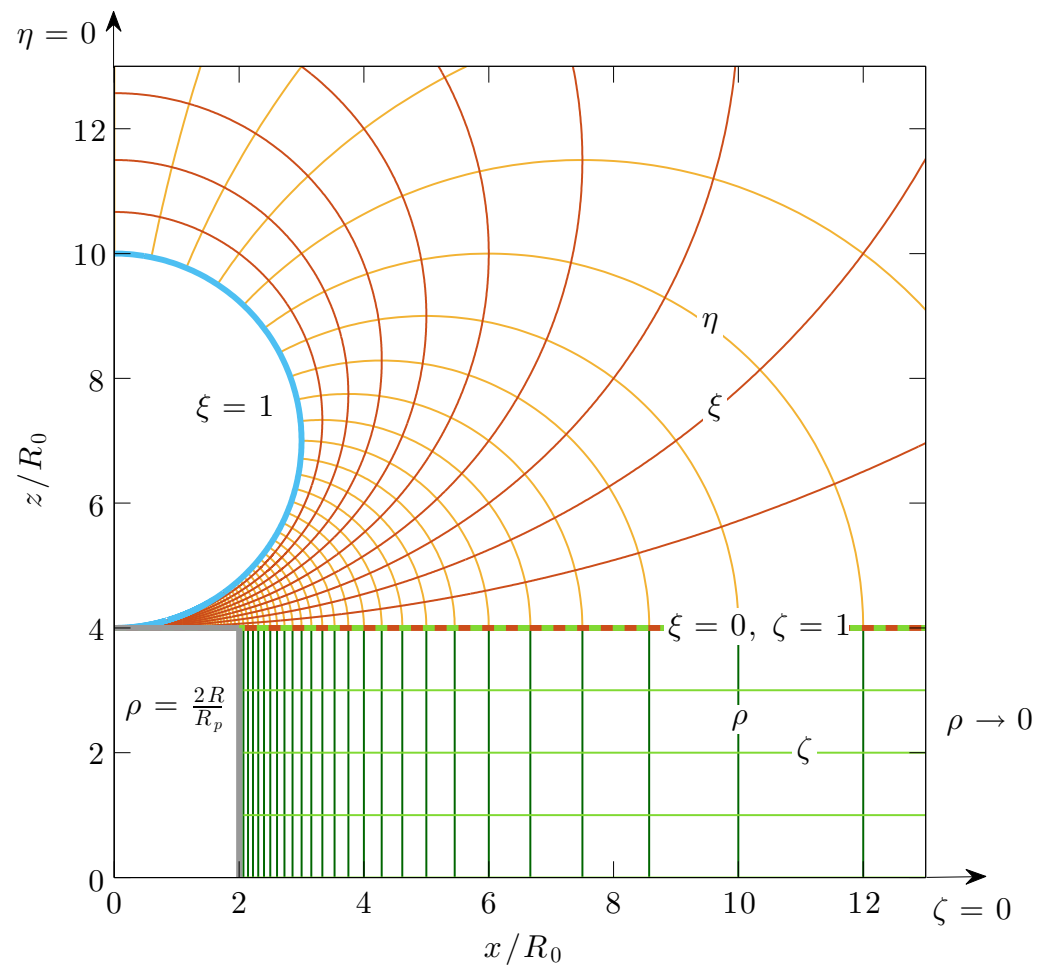

Figure 4.5: Schematic of the simulation grid for a pillar with dimensions $H_{p} / R_{0}=4$ and $R_{p} / R_{0}=2$. The simulation points fall on the intersection of the contourlines. The simulation is axisymmetric around the $z$-axis. The bulk region in tangent-sphere coordinates $(\eta, \xi)$ applies to $z>H_{p}$. The microlayer region in pseudo-cylindrical coordinates $(\rho, \zeta)$ applies to $0<$ $z<H_{p}$. A matching condition is imposed at $z=H_{p}$. Coordinates $\eta$, $\xi$ and $\rho$ evolve with the bubble, so that $\xi=1$ (in blue) always maps the bubble surface, regardless of the actual bubble size. Here, the bubble size has been arbitrarily set to $R / R_{0}=3$. The separation of the contours is uniform. Here, $\Delta \eta=\Delta \xi=\Delta \rho=0.1$ and $\zeta=0.5$. The coordinate $\eta=0$ lies on the $z$-axis, $\eta \rightarrow \infty$ at the contact point. 
of $\mathrm{H}_{2}$. Nonetheless, the small molar fractions of air typically present in the bubbles $(<15 \%)$ can be neglected for all other purposes.

\subsection{Results and discussion}

\subsubsection{Significance of the diffusive growth coefficient}

Assuming diffusion-limited growth, the $n^{\text {th }}$ bubble in the succession grows as $R_{n}\left(t_{n}\right)=b_{n} \sqrt{t_{n}}$, where $b_{n}$ is its associated diffusive growth coefficient, and $t_{n}=t-t_{0, n}$ is the current time after nucleation at $t=t_{0, n}$. In spirit of the theories for monocomponent bubble growth by Epstein et al. (1950)[14] or Scriven (1959)[101], it is convenient to recast this law in terms of the dimensionless growth coefficient $\tilde{b}_{n}=b_{n} / \sqrt{D}$ such that

$$
R_{n}\left(t_{n}\right)=\tilde{b}_{n} \sqrt{D t_{n}},
$$

where $D$ denotes the gas diffusivity. Indeed, if the bubble grows to a radius $R_{n}$ much larger than the size of the nucleation site (the pit on the pillar in our case), its growth will be given by the Epstein-Plesset equation [14], that determines the quasi-static diffusive growth of an isolated spherical bubble:

$$
\frac{\mathrm{d} R_{n}}{\mathrm{~d} t_{n}}=\frac{D R_{u} T_{\infty}}{P_{0}}\left(\bar{C}_{n}-k_{H} P_{0}\right)\left[\frac{1}{R_{n}}+\frac{1}{\sqrt{\pi D t_{n}}}\right] .
$$

In this expression, $R_{u}$ and $k_{H}$ are the universal gas and Henry's constants, respectively. Moreover, $T_{\infty}$ and $P_{0}$ are the liquid temperature and pressure during growth, while $\bar{C}_{n}$ is the gas concentration far away from the bubble. Finally note that surface tension has been neglected, as even the largest capillary (or Laplace) pressure $2 \gamma_{l g} / R \sim 0.05$ bar is negligible in comparison to the pressure involved in bubble growth, $P_{0} \sim 8$ bar. Here, we have considered surface tension $\gamma_{l g}=0.07 \mathrm{~N} / \mathrm{m}$ and the smallest trackable bubble size, $R=30 \mu \mathrm{m}$.

In the case of electrolysis within our air-equilibrated electrolyte, the Epstein-Plesset equation will be valid for sufficiently large concentrations of $\mathrm{H}_{2}$ such that the flux of other species $\left(\mathrm{N}_{2}, \mathrm{O}_{2}\right)$ into the growing bubble is small in comparison. In such cases, the ratio of $\mathrm{H}_{2}$-to-air fluxes can be shown to be roughly equivalent to where $\zeta$ is the supersaturation of $\mathrm{H}_{2}$, to be defined in (4.7b).

At times $t_{n} \gg R_{0}^{2} / D$ - this corresponds to roughly $t_{n} \gtrsim 1 \mathrm{~s}$ in our experiments - equation (4.4) allows for the following solution (neglecting surface tension):

$$
R_{n}\left(t_{n}\right) \approx \sqrt{J a_{n} / \pi}\left[\sqrt{J a_{n}}+\left(2 \pi+J a_{n}\right)^{1 / 2}\right] \sqrt{D t_{n}} .
$$


Table 4.1: Gas properties in our experimental conditions $\left(T_{\infty}=20^{\circ} \mathrm{C}\right)$.

\begin{tabular}{llll}
\hline & $\mathrm{CO}_{2}$ & $\mathrm{H}_{2}$ & Air $^{c}$ \\
\hline$D\left(\mathrm{~m}^{2} / \mathrm{s}\right)$ & $1.78 \times 10^{-9} a$ & $4.2 \times 10^{-9 b}$ & $2.0 \times 10^{-9 a}$ \\
$k_{H}\left(\mathrm{~mol} \mathrm{~m}^{-3} \mathrm{~Pa}^{-1}\right)$ & $3.36 \times 10^{-4}$ & $7.7 \times 10^{-6}$ & $8.5 \times 10^{-6}$ \\
$\Lambda$ & 0.824 & 0.0188 & 0.0207 \\
\hline
\end{tabular}

${ }^{a}$ supersaturated pressure $\approx 8 \mathrm{bars} ;{ }^{b}$ ambient pressure: $1 \mathrm{bar} ;{ }^{c}$ air is only considered in the simulation.

This solution is essentially that provided by Epstein et al. (1950)[14] formulated for the $n^{\text {th }}$ bubble in the succession and, correspondingly, using the mass-transfer Jakob number $[174,186]$ related to the $n^{\text {th }}$ bubble,

$$
J a_{n}=\frac{R_{u} T_{\infty}}{P_{0}}\left(\bar{C}_{n}-k_{H} P_{0}\right)=\Lambda \zeta_{n} .
$$

The Jakob number is a measure of the driving force for bubble growth induced by a solubility parameter $\Lambda$, commonly known as the Ostwald coefficient, and the degree of supersaturation $\zeta_{n}$. These are defined as follows:

$$
\Lambda=k_{H} R_{u} T_{\infty}, \quad \zeta_{n}=\frac{\bar{C}_{n}}{k_{H} P_{0}}-1 .
$$

We expect the Jakob number to be smaller than unity in our experiments. Indeed, for the P-C experiments, multiplying the supersaturation of $\mathrm{CO}_{2}$ ( $\zeta_{n} \approx 0.17$ ) by the corresponding solubility tabulated in Table 4.1 results in $J a_{n} \approx 0.14$. For the $\mathrm{H}_{2}$ electrolysis experiments, $\zeta_{n}<12$ according to simulations and therefore $J a_{n}<0.23$. Equation (4.5) then simplifies to

$$
R_{n}\left(t_{n}\right)=\sqrt{2 J a_{n} D t_{n}}
$$

since $J a_{n} \ll 2 \pi$ always. Within this diffusive regime, the Jakob number can be readily shown to be equivalent to the Péclet number, $P e_{n}=R_{n} \dot{R}_{n} / D$, which expresses the relative significance of advective over diffusive mass transport. The quantity $\dot{R}_{n}=\mathrm{d} R_{n} / \mathrm{d} t_{n}$ can be computed by differentiating (4.8) with respect to time. One then gets that $P e_{n} \equiv J a_{n}$, which is consistent with the fact that the Péclet number must also be smaller than unity. Finally, direct comparison of (4.8) with (4.3) gives that the square of the dimensionless growth coefficient scales as the Jakob number:

$$
\tilde{b}_{n}^{2}=2 J a_{n}=2 \Lambda \zeta_{n} .
$$

It should be pointed out that, strictly speaking, (4.9) is only valid for a spherical bubble far from any boundary. Nonetheless, taking into account the presence of the substrate will not modify any of the scalings, but will 
only change the numerical coefficient relating $\tilde{b}_{n}^{2}$ and $J a_{n}$, as shown by Enríquez et al. (2014)[113] for P-C growth. In P-C growth, this coefficient is expected to be lowered by the presence of the inert substrate which has a hindering effect on bubble growth $[113,187]$. This is perceived upon inspection of the $\mathrm{CO}_{2}$ concentration field displayed in Figure 4.6 $(d)$ obtained from simulation. The concentration isocontours are farther apart closer to the substrate compared to those near the apex.

The opposite occurs for the first bubbles in the case of electrolysis-driven growth, where $\mathrm{H}_{2}$ transfer rates (concentration gradients) are highest in the portion of bubble interface located closest to the electrode, as can be inferred from the accumulation of iso-concentration lines in the region closer to the substrate (see Figures $4.6 a, b$ ). Here, the bubbles are set to nucleate shortly after the onset of electrolysis and consequently the $\mathrm{H}_{2}$ supersaturation in the bulk electrolyte is still low. One may note that as the degree of supersaturation in the bulk liquid increases with time (Figure $4.6 a$ to $4.6 b$ to $4.6 c$ ), the boundary layer evolves to the form attained in the P-C case (Figure 4.6d). Most of the $\mathrm{H}_{2}$ flux occurs then at the apex of the bubble, as observed for P-C growth.

Finally, we must remark that the pillar height $H_{p}$ is much smaller than the radius of the growing bubble except for the initial seconds of bubble growth. Therefore, the effect of the pillar height on the aforementioned coefficient is expected to be small. 

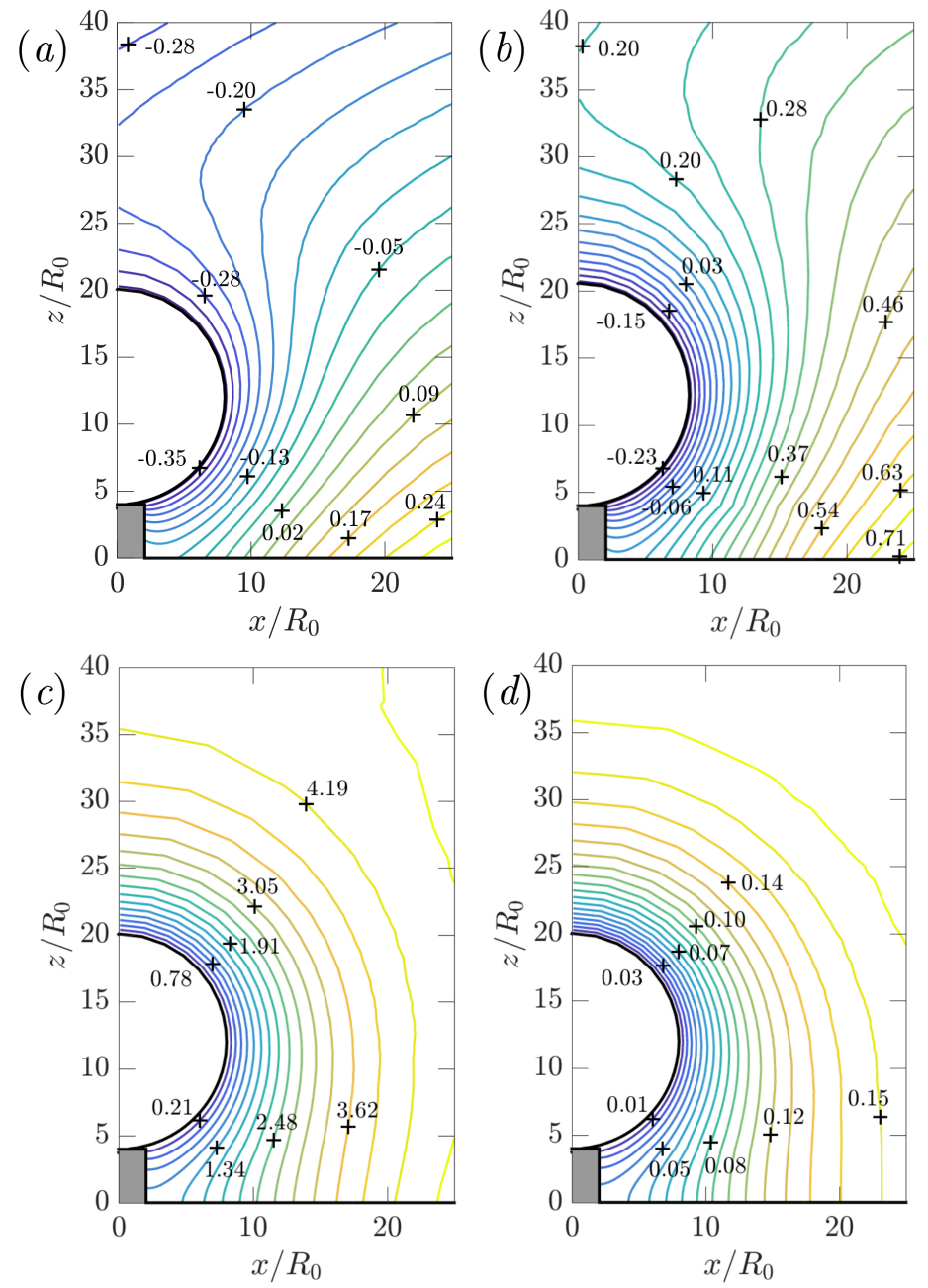

Figure 4.6: Simulation snapshots of $(a-c) \mathrm{H}_{2}$ or $(d) \mathrm{CO}_{2}$ concentration isocontours around four distinct growing bubbles. The contour labels display the local supersaturation value, $C /\left(k_{H} P_{0}\right)-1$. The simulations are performed for $R_{0}=7.5 \mu \mathrm{m}, R_{p}=15 \mu \mathrm{m}$ and $H_{p}=30 \mu \mathrm{m}$. The snapshots correspond to the early growth stage of the bubbles when $R=2 H_{p}=60$ $\mu \mathrm{m}$. Panels $(a-c)$ show $\mathrm{H}_{2}$ bubbles growing in an air-saturated electrolyte assuming an effective current density of $1.56 \mathrm{~A} / \mathrm{m}^{2}$ that provides a similar $\mathrm{H}_{2}$ flux as the highest nominal current density used in our experiments (see later Fig. 4.13.) The bubble nucleation time after the start of electrolysis, $t_{0}$, the corresponding initial degree of $\mathrm{H}_{2}$ supersaturation (in the absence of bubbles), $\zeta$, and time after nucleation at which the snapshot is taken, $t$, are (a) $t_{0}=30 \mathrm{~s}, \zeta \approx 0, t \approx 31 \mathrm{~s},(b) t_{0}=2 \mathrm{~min}, \zeta \approx 1, t \approx 16 \mathrm{~s},(c) t_{0}=20 \mathrm{~min}$, $\zeta \approx 5.3, t \approx 3 \mathrm{~s}$. Panel $(d)$ portrays a $\mathrm{CO}_{2}$ bubble growing in an initially uniform supersaturated solution, $\zeta=0.17$ at $P_{0}=7.75$ bars. The bubble is $t \approx 5 \mathrm{~s}$ old. 


\subsubsection{Growth dynamics}

The experimental diffusive growth coefficient for each electrolytic bubble in the succession was obtained by fitting the experimental data to (4.3), namely $R_{n}\left(t_{n}\right)=\tilde{b}_{n} \sqrt{D t_{n}}$. More specifically,

$$
\tilde{\beta}_{H_{2}{ }^{2}}=\frac{1}{D_{H_{2}}}\left\langle\frac{\mathrm{d} R^{2}}{\mathrm{~d} t}\right\rangle_{R \rightarrow R_{d}} .
$$

Here, $\tilde{\beta}_{H_{2}}$ is the asymptotic growth coefficient [173] at which the bubble reaches its detachment size. Even so, $\tilde{\beta}_{H_{2}}$ should be regarded, by all accounts, as the experimentally-obtained $\tilde{b}$ for the electrolytic bubbles.

(a)

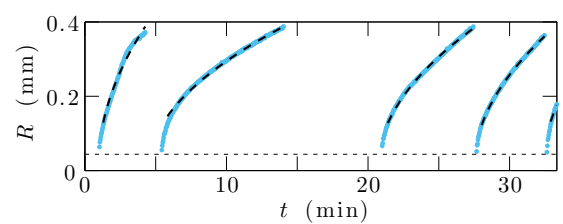

(c)

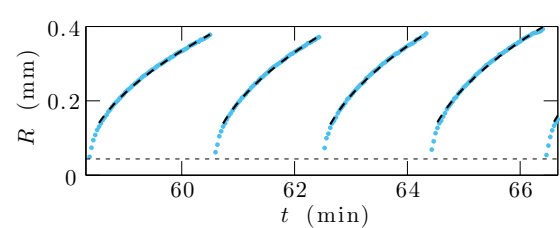

(e)

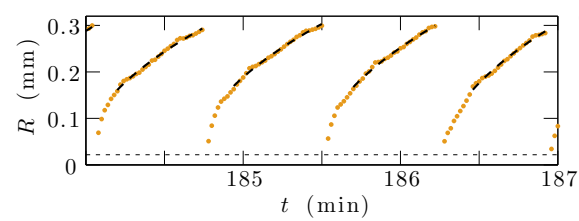

(b)

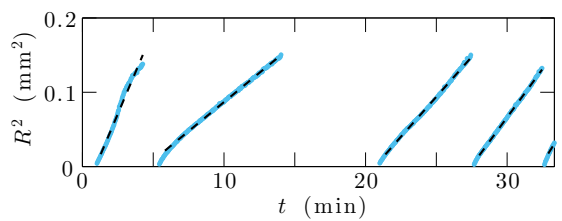

$(d)$
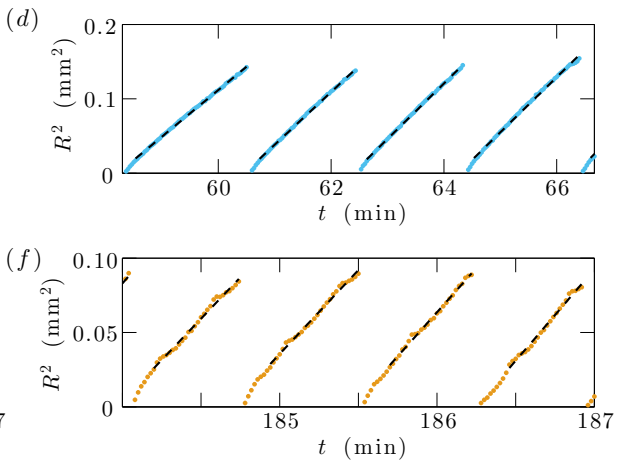

Figure 4.7: Bubble radius dynamics at three different time periods after the onset of electrolysis with $7.8 \mathrm{~A} / \mathrm{m}^{2}$. The measured bubble radii (dots) are plotted in left-column panels $(a, c, e)$ whereas the corresponding square of the measured radii are plotted in the right-column panels $(b, d, f)$. Panels $(a, b)$ correspond to the stagnation regime, $(c, d)$ to the supersaturation regime and $(e, f)$ to the advected growth regime. The dashed black curves are the fits from which the asymptotic growth coefficient $\tilde{\beta}_{H_{2}}$ is obtained. The pit and pillar radius are 5 and $15 \mu \mathrm{m}$, respectively. The plots in $(a)-(d)$ belong to the same experiment where $H_{p}=30 \mu \mathrm{m}$. The plots in $(e, f)$ belong to a different experiment where $H_{p}=15 \mu \mathrm{m}$. The dotted horizontal line marks the size below which it was not possible to optically determine the size of the bubble accurately.

The theoretical fits are plotted in Figure 4.7 for three different time periods after the start of electrolysis. Three regimes with distinct growth 
dynamics can be identified depending on the elapsed time of electrolysis. At early times, we devise a characteristic 'stagnation regime' (see Figure $4.7 a$ ), in which the second and third bubble usually grow slower than the very first. Here, the accumulation of $\mathrm{H}_{2}$ gas that one would expect from the continuous flux at the electrode is in fact exhausted or overridden by local gas depletion effects induced by the growth of the first bubbles and other sources that will be later discussed. The duration of this stagnation regime is shortened with increasing current density (see later Figure 4.9). It stands to reason that no depletion regime may be observed if the current density is sufficiently large.

Thus, provided that the current density is large enough, the flow of $\mathrm{H}_{2}$ into the bubble liquid surroundings eventually overrides the flow of gas lost through depletion effects. This results in a net accumulation of $\mathrm{H}_{2}$ and, consequently, a vertical $\mathrm{H}_{2}$ concentration boundary layer that develops from the electrode.

In such a case, a supersaturation regime (Figure 4.7c) is eventually attained. The local supersaturation of the surrounding electrolyte is now more uniform. The expected diffusive behaviour, $R_{n}^{2} \propto t_{n}$, can be corroborated in Figure 4.7(d). If, at later times, the supersaturation becomes large enough, an advective growth regime (Figure 4.7e) becomes manifest. It is characterised by discontinuous $\mathrm{d} R / \mathrm{d} t$ dynamics caused by the appearance of boundary-induced advection in bubble growth and departure [182]. Furthermore, a fast initial growth is observed. It is attributed to the presence of a thin boundary layer of excess $\mathrm{H}_{2}$ gas near the gas-evolving electrode that quickly gets adsorbed into the bubble. In consequence, the initial growth is observed to be more pronounced for pillar heights of 0 or $15 \mu \mathrm{m}$ as opposed to $30 \mu \mathrm{m}$.

In slightly supersaturated water- $\mathrm{CO}_{2}$ solutions, bubbles are known to diffusively grow before density-driven convection sets in at the final stages of their growth [113]. In consequence, the diffusive growth coefficient for the $\mathrm{CO}_{2}$ bubbles in the P-C experiments was similarly computed as

$$
\tilde{\beta}_{C O_{2}}^{2}=\frac{1}{D_{C O_{2}}}\left\langle\frac{\mathrm{d} R^{2}}{\mathrm{~d} t}\right\rangle_{R<3 R_{d} / 4},
$$

where $R_{d}$ denotes the bubble radius at detachment. The upper limit $3 R_{d} / 4$ is established in order to restrict the fitting of $\tilde{\beta}_{\mathrm{CO}_{2}}$ to just the initial diffusive growth regime.

The theoretical fit (extrapolated to span the full bubble lifetime) is compared to a typical experiment $(\zeta=0.17)$ in Figure 4.8. The initial diffusive growth, where $R \propto \sqrt{t}$, can be perfectly distinguished. One may also note a deviation between the theoretical fits and experiments as the bubble radius reaches its detachment size. This deviation is expected 
since it is related to the onset of natural convection at the end of bubble growth. The prominent effect of density-induced convection on bubble growth observed here is, on the other hand, negligible for the case of $\mathrm{H}_{2}$ bubbles.
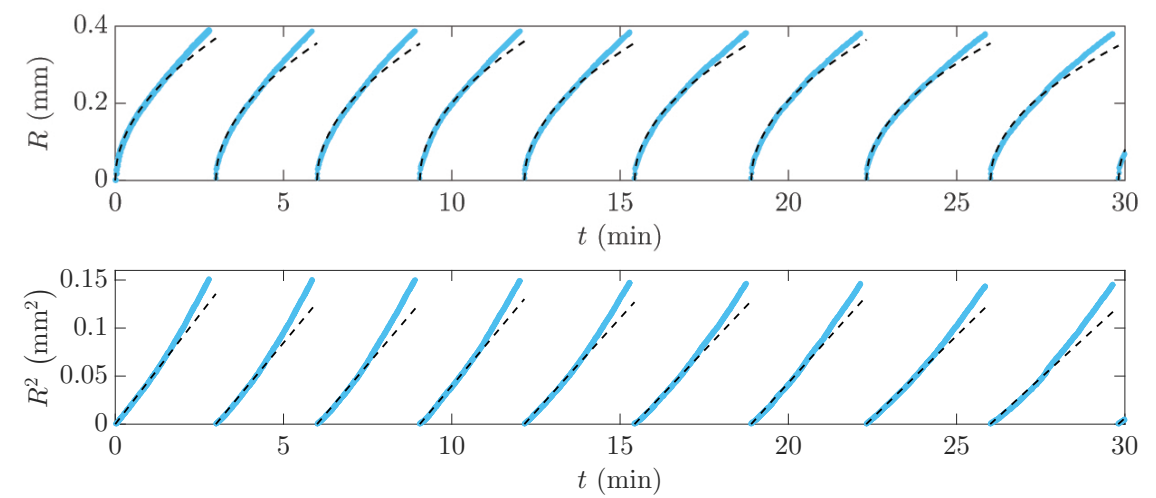

Figure 4.8: (a) Pressure-controlled bubble radius dynamics and (b) the corresponding squared radius. The dashed curves are the (extrapolated) fits from which $\widetilde{\beta}_{\mathrm{CO}_{2}}$ is computed. The pit and pillar radius are 10 and 15 $\mu \mathrm{m}$, respectively, whereas the pillar height is $30 \mu \mathrm{m}$.

The computation of $\tilde{\beta}_{\mathrm{H}_{2}}$ and $\tilde{\beta}_{\mathrm{CO}_{2}}$ for each individual bubble essentially allows for a quantitative measure of the evolution of the growth rate of the bubble succession as a whole. The fact that the evolution of $\tilde{\beta}_{H_{2}}$ in time for a succession of bubbles under constant-current electrolysis is far from uniform is immediately corroborated in Figure 4.9. The first few bubbles fall under what we have referred to as the stagnation regime, in which $\tilde{\beta}_{H_{2}}$ is observed to decrease or remain fairly constant. The stagnation regime occurs due to the partial depletion of the evolving boundary layer of dissolved gas. There are three main sources of depletion that will be soon described. These are responsible for the ever-present loss of gas dissolved in the cavity where the electrode is held (cf. Figure 4.2). This loss is most noticeable and even dominant at the early stages of electrolysis. This stands to reason since, as mentioned before, the local concentration of dissolved $\mathrm{H}_{2}$ gas near the pillar is relatively small at the beginning of the electrolysis. Accordingly, gas losses due to depletion constitute a proportionally large reduction of the small amount of gas dissolved.

The subsequent bubbles experience a fast increase in $\tilde{\beta}_{H_{2}}$. This is a sign of the net accumulation of dissolved $\mathrm{H}_{2}$ gas in the cavity. In other words, the flow rate of evolved $\mathrm{H}_{2}$ into the cavity overrides the smaller flow rate losses due to depletion. Figure 4.10 displays the same experimental values of $\tilde{\beta}_{H_{2}}$ as function of the total electric charge that has been in circulation before a given time, which is a direct measure of the total mass of $\mathrm{H}_{2}$ 


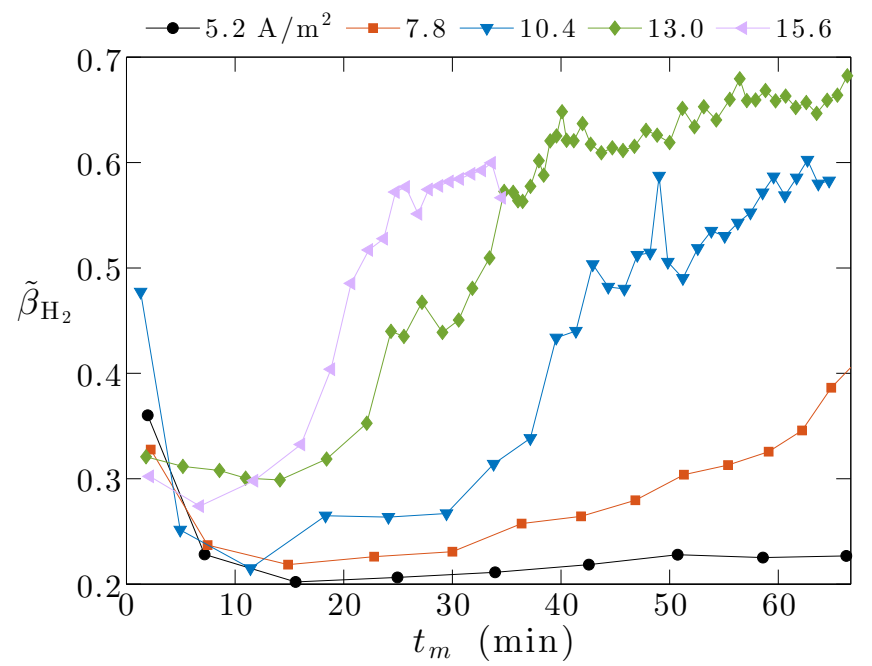

Figure 4.9: $\tilde{\beta}_{H_{2}}$ for a succession of electrolytic bubbles exposed to constant current densities $\left(I_{w}\right)$. Each point corresponds to a distinct bubble in the succession. Time $t_{m}$ is the mean time (after the start of electrolysis) of the fitting period used to compute $\tilde{\beta}_{H_{2}}$. The bubbles evolved on a pit with a radius of $5 \mu \mathrm{m}$, a pillar radius of $15 \mu \mathrm{m}$ and a pillar height of $30 \mu \mathrm{m}$.

supplied. For a given charge $Q=I_{w} t$, during the initial transient stage of electrolysis, we conclude that $\tilde{\beta}_{H_{2}}$ is higher for larger current densities. It is expected that the gas evolution efficiency increases with current density since the diffusion length of dissolved $\mathrm{H}_{2}$, which scales as $\sqrt{D_{H_{2}} Q / I_{w}}$, is smaller. In other words, mass is supplied faster and $\mathrm{H}_{2}$ does not diffuse fast enough into the bulk electrolyte above the cavity in which the bubble evolves. Consequently, the gas supersaturation in the cavity surrounding the electrode is higher.

Naturally, in the P-C experiments, the absence of gas production must derive into a permanent depletion regime. Moreno Soto et al. (2017)[103] recently developed a theoretical depletion model for this particular scenario. Based on this model (see Appendix B), the evolution of the experimental growth coefficient should approximately satisfy

$$
\frac{1}{\tilde{\beta}_{C O_{2}}^{2}(t)}-\frac{1}{\tilde{\beta}_{C O_{2}}^{2}(0)}=\frac{2 \pi}{3} \frac{D_{C O_{2}} t}{R_{d}^{2}} .
$$

The experimental evolution in time of $\tilde{\beta}_{\mathrm{CO}_{2}}$ in plotted in Figure 4.11(a). In Figure 4.11(b), these are compared to the best fitting law $1 / \tilde{\beta}_{\mathrm{CO}_{2}}^{2}(t)=$ $m t+c$. According to (4.12), the slope $m=0.027 \mathrm{~s}^{-1}$. This estimate agrees well with experiments, where $m \approx 0.03 \mathrm{~s}^{-1}$. Similarly, the constant $c=2.93$ 


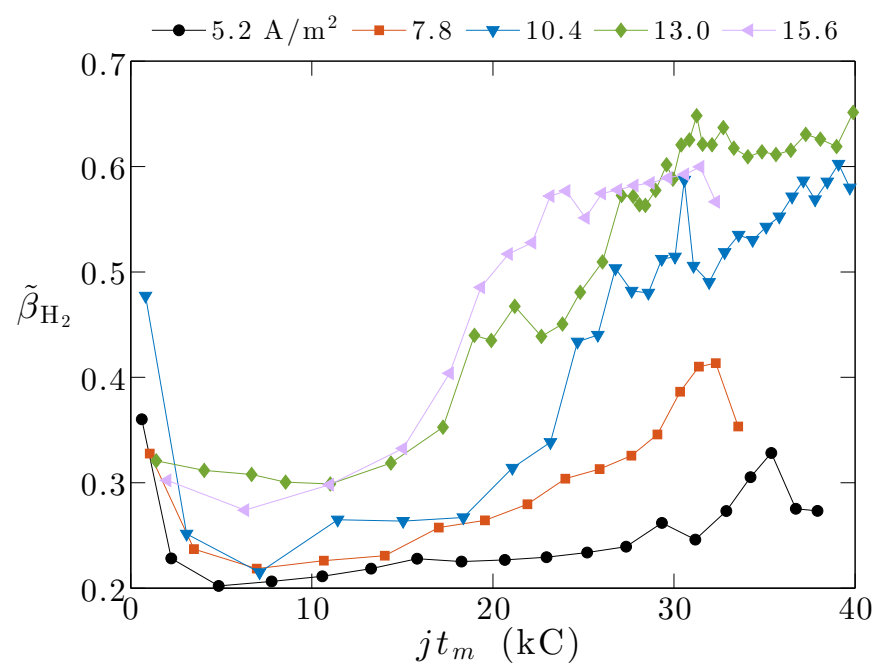

Figure 4.10: Diffusive growth coefficient as a function of charge for different current densities (see caption of Figure 4.9). The efficiency of gas evolution in the transient regime increases with the current density $I_{w}$.

from theory falls close to the experimental values which lie within 2.6-2.9. The small differences may be associated to the many simplifications and approximations that the theoretical model is based upon. Nonetheless, the good overall agreement between the model and experiments indeed suggests that in the P-C configuration there is only depletion from previous bubbles in the succession. 

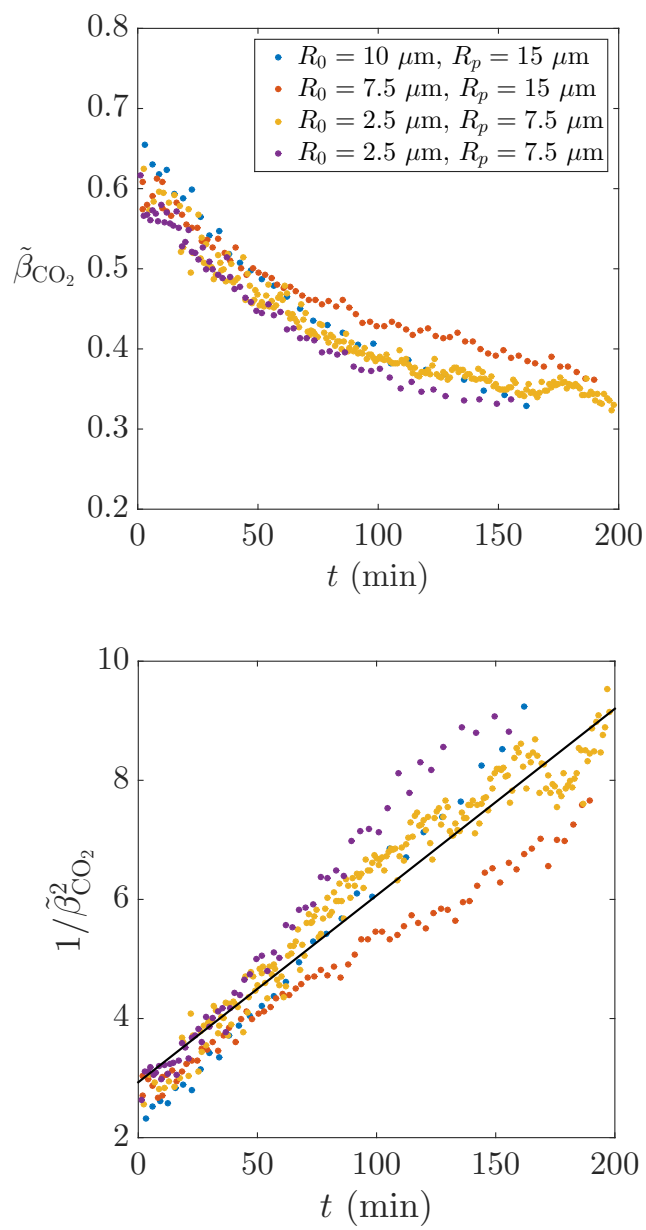

Figure 4.11: (a) Diffusive growth coefficient, $\tilde{\beta}_{\mathrm{CO}_{2}}$, as a function of time for four different P-C experiments. The time measurement for each bubble $n$ is associated to its moment of detachment. (b) Plot of $1 / \tilde{\beta}_{\mathrm{CO}_{2}}^{2}$ as a function time. The straight solid line shows the linear relation (4.12), with a slope $m=2 \pi D_{\mathrm{CO}_{2}} t /\left(3 R_{d}^{2}\right)=0.027 \mathrm{~s}^{-1}$. 


\section{Depletion sources in the electrolysis experiments}

In our electrolysis experiments, however, the electrolyte in the cavity suffers $\mathrm{H}_{2}$ gas depletion from (i) previous bubbles in the succession, from (ii) parasitic bubbles (eventually mm-sized) forming all around the sidewall of the circular cavity, and from (iii) the cavity opening, toward which the $\mathrm{H}_{2}$ is transported by diffusion. Regarding the latter, it is worth noticing that the cavity perimeter is much smaller than the perimeter of the holder above (cf. Figure 4.2). In addition, the volume of electrolyte in the tank above the cavity is almost three orders of magnitude bigger than the volume of the cavity itself. The opening of the cavity therefore initially acts as a zero $\mathrm{H}_{2}$ concentration boundary condition (a pseudo free surface) since $\mathrm{H}_{2}$ is essentially diffusing into an infinite reservoir.

The effect of these depletion sources on the bubble growth dynamics will be discussed next for the electrolysis experiments, discerning between the stagnation regime and supersaturation (eventually steady-state) regime that follows.

\section{Stagnation regime}

In the stagnation regime, the bulk concentration of dissolved $\mathrm{H}_{2}$ gas within the cavity is small. We can then make the rough approximation that the $\mathrm{H}_{2}$ gas in these bubbles is entirely supplied by the molar flux $J_{w}$ that is evolved within a harvest area $\delta^{2}$ on the flat electrode. The characteristic harvest length, $\delta$, depends of course on the aforementioned depletion sources. Indeed, $\delta$ represents an important design parameter in electrolytic systems which should be investigated in future studies. Assuming that these bubbles are entirely composed of $\mathrm{H}_{2}$, a simple mass balance scaling yields

$$
\frac{P_{0}}{R_{u} T_{\infty}} \frac{R_{d}^{3}}{\Delta t} \sim J_{w} \delta^{2}
$$

where $\Delta t$ denotes the bubble lifetime prior to detachment. As a first approximation, we expect $\delta \sim R_{d}$. If we further assume purely diffusive growth, $R_{d}=\tilde{\beta}_{H_{2}} \sqrt{D_{H_{2}} \Delta t}$, and noting that $J_{w} \propto I_{w}$, then (4.13) suggests that the asymptotic growth rate must scale as $\tilde{\beta}_{H_{2}}^{2} \sim I_{w} R_{d}$. This relation is compared against experimental values in Figure 4.12. The experimental dispersion is expected due to the random variability of the harvest length (primarily due to the spatial non-uniformity of the $\mathrm{H}_{2}$ flux at the electrode) and depletion strength from one experiment to the other.

Within the stagnation regime or transient regime that follows, the diffusive depletion at the cavity opening has an important retardation effect on the bubble growth rate as the bubble grows bigger and gets closer to the opening. This retardation can be noted upon inspection of Figures 4.3 


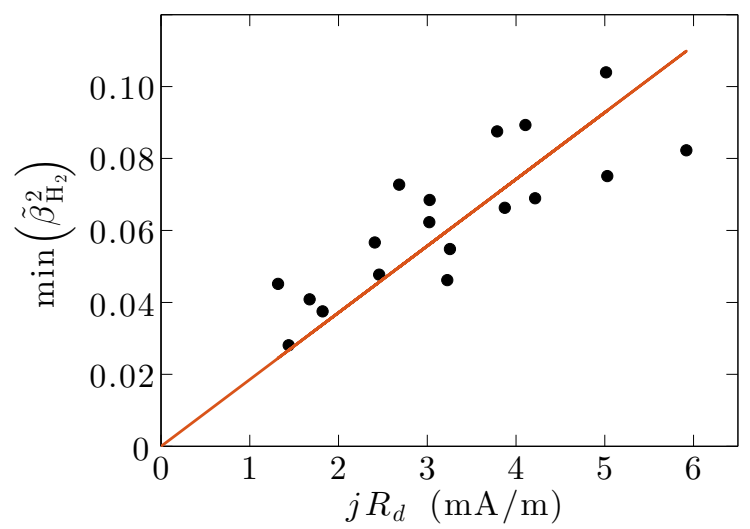

Figure 4.12: Minimum squared value of $\tilde{\beta}_{H_{2}}$ plotted versus the product of current density and detachment radius. Each point corresponds to a single bubble in a unique succession. The linear regression fit is also plotted.

and 4.7(a). This claim is supported by numerical simulations as seen in Figure 4.13. The simulation curves predict the experimental behavior once a zero-concentration boundary condition is imposed at a height somewhat lower, but close to the actual cavity depth of $2 \mathrm{~mm}$. However, this is expected, since a lower height also accounts for other losses such as parasitic bubble formation at the cylindrical sidewall of the cavity.

It is imperative to note that the effective current density (which establishes the molar flux of dissolved $\mathrm{H}_{2}$ at the electrode) employed in the simulation is in fact taken as a free parameter. The effective current density, together with the experimental time of nucleation of a particular bubble are put into (4.17) in Appendix A to obtain the initial concentration field in which that particular bubble grows. The effective current density is thus chosen so that the numerical solution matches the experimental radii at the initial growth stage of the bubble. Surprisingly, the effective current densities are 7 to 10 times smaller than the nominal (experimental) values. Accordingly, the growth rates obtained by simulations at the nominal current densities were exceedingly high. A similar discrepancy in theoretical versus measured $\mathrm{H}_{2}$ diffusive fluxes was observed by Aoki et al. (2012) [188], who argued that most of the gas may not actually dissolve in hydrated form. They reported that $\mathrm{H}_{2}$ gas preferentially resides in the form of stable bulk nanobubbles $(R \sim 400 \mathrm{~nm})$ instead $[189,188]$.

Although we cannot give at the moment a precise answer to this discrepancy, we propose two plausible - not mutually excluding explanations. Firstly, not all the $\mathrm{H}_{2}$ flux put in dissolution remains in the electrolyte or eventually goes to the bubble nucleating at the pit. Indeed, 
- experiment -w/ cavity effect ---w/o cavity effect
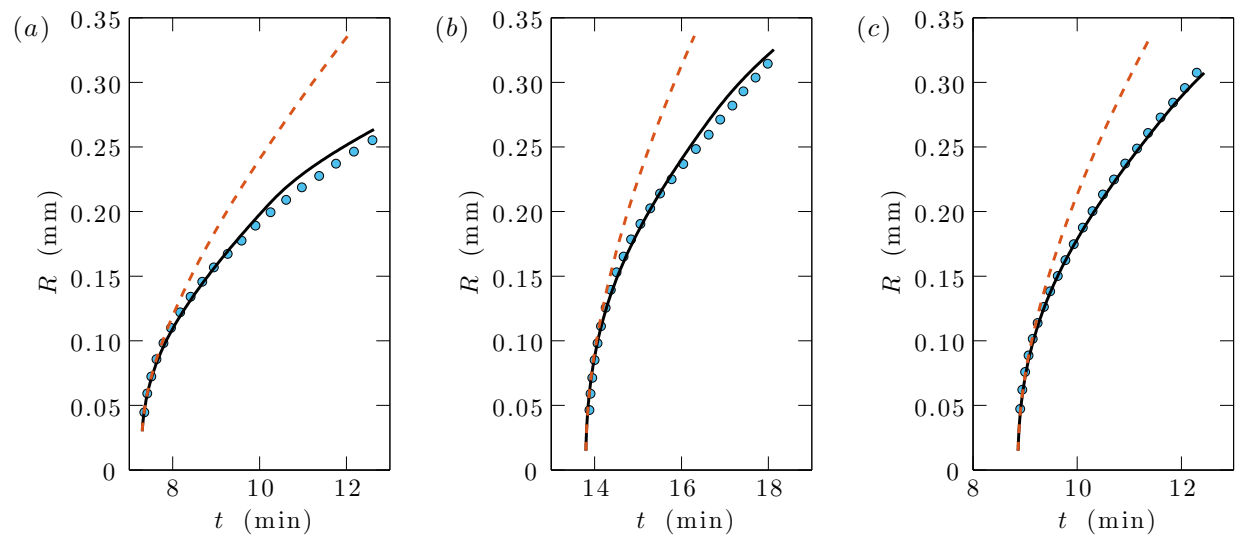

Figure 4.13: Comparison between simulation (curves) and the experimental growth dynamics of the third bubble for three nominal current densities: (a) 7.8, (b) 10.4 and (c) $15.6 \mathrm{~A} / \mathrm{m}^{2}$. Simulations employ effective current densities of $(a)$ 1.04, (b) 1.30 and (c) $1.56 \mathrm{~A} / \mathrm{m}^{2}$. Using (4.17) in Appendix A, these yield initial supersaturations, $\zeta$, of approximately (a) $1.5,(b) 3.3$ and (c) 3.1. The black curves are computed imposing a zero-concentration boundary condition at height of $(a) 1.0 \mathrm{~mm},(b) 1.3 \mathrm{~mm}$ and $(c) 1.3 \mathrm{~mm}$ to model the effect of the cavity. The red dashed curves are computed without imposing the aforementioned boundary condition.

we observe that large bubbles unavoidably form at the cylindrical cavity sidewall. Secondly, the numerical model assumes a spatially uniform current density along the substrate. In practice, this may not be so. In fact, current density can be highly non-homogeneous for planar electrodes [160]. Moreover, one may note that the actual boundary condition occurring at the electrode is of constant potential, and not of uniform current density. Thus, close to the base of the pillar the local current density must be smaller than the average on the electrode surface.

\section{Supersaturation regime}

In the supersaturation regime, the gas concentration in the cavity is relatively more uniform at the beginning of the bubble growth, especially for sufficiently high supersaturations, $\bar{C}_{n} /\left(k_{H} P_{0}\right) \gg 1$. Making then use of (4.6), $\bar{C}_{n}$ can be made continuous in time by setting $\bar{C}(t)$ equal to the wall concentration $C(z=0, t)$ in the absence of bubbles assuming a 1D profile. The expression is provided in (4.17) in Appendix A. In such a case, 
the growth coefficient has the following behavior at long times:

$$
\tilde{\beta}_{H_{2}}^{2}(t) \approx \frac{2 R_{u} T_{\infty}}{P_{0}} \bar{C}\left(t ; H_{e f f}, J_{w, e f f}\right) .
$$

The local concentration $\bar{C}(t)$ depends on the effective molar flux $J_{w, e f f}$, or, equivalently, on the effective current density $I_{w, \text { eff }}$. These will be smaller than the nominal values in order to account for the aforestated discrepancy between the experimental and theoretical concentrations. Furthermore, $\bar{C}(t)$ is also dependent on the effective electrolyte liquid column height $H_{\text {eff }}$ which is expected to be lower than $H=12 \mathrm{~mm}$. $H_{\text {eff }}$ accounts for the geometry of the electrolysis holder and cavity and losses from growing and departing bubbles at the cavity perimeter. For high current densities, $H_{\text {eff }}$ is also limited by the maximum supersaturation of dissolved $\mathrm{H}_{2}$ that the electrolyte can withstand at the experimental pressure. The steady-state growth coefficient can be shown to directly depend on these two quantities:

$$
\tilde{\beta}_{H_{2}, s s}^{2} \approx \frac{2 R_{u} T_{\infty} F v}{D_{H_{2}} P_{0}} I_{w, \text { eff }} H_{\text {eff }} .
$$

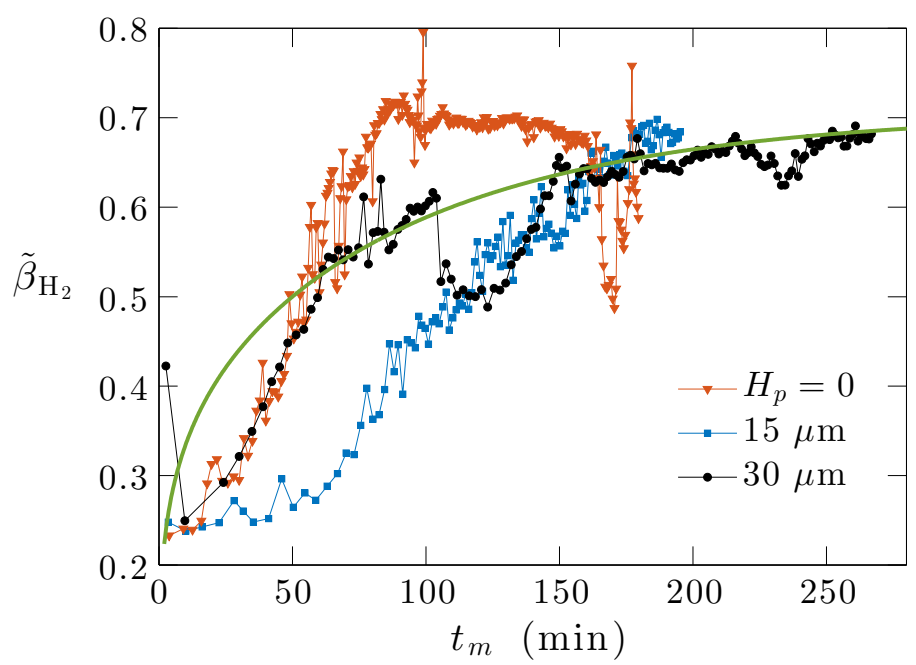

Figure 4.14: Evolution in time of the diffusion growth rate for $I_{w}=7.8$ $\mathrm{A} / \mathrm{m}^{2}$ for three different experiments. The pit radius is $R_{0}=5 \mu \mathrm{m}$ for $H_{p}=0$ and $30 \mu \mathrm{m}$, whereas $R_{0}=1 \mu \mathrm{m}$ for $H_{p}=15 \mu \mathrm{m}$. The green curve is a theoretical approximate solution given in (4.14), with $I_{w, \text { eff }}=1.04 \mathrm{~A} / \mathrm{m}^{2}$ (same value as in simulations) and $H_{\text {eff }}=8 \mathrm{~mm}$.

Figure 4.14 compares this theoretical model for the behavior of $\tilde{\beta}_{H_{2}}$ at long times against three experiments with different pillar heights but 
performed under the same current density. A similar steady-state value is attained in all cases after about $150 \mathrm{~min}$. However, it is evident that the transient behaviour of $\tilde{\beta}_{H_{2}}$ is not repetitive across experiments. The deviations arise presumably due to differences in the flux distribution across the electrode samples and especially due to gas losses from the random formation and subsequent detachment of large parasitic bubbles on the cavity sidewall. The bubbles in the succession must then compete against the parasitic bubbles for the available $\mathrm{H}_{2}$ gas. The formation of the parasitic bubbles ultimately has a hindering effect on the growth rates of the bubbles growing on the pillar. This effect increases over time as the solution becomes more supersaturated and the parasitic bubbles increase in size and hence in proximity to the micropillar. For instance, the sudden decrease in $\tilde{\beta}_{H_{2}}$ at around $110 \mathrm{~min}$ in the experiment with $H_{p}=30 \mu \mathrm{m}$ in Figure 4.14 is a consequence of a particular large parasitic bubble that expanded quite close to the pillar. After its detachment at approximately $130 \mathrm{~min}, \tilde{\beta}_{H_{2}}$ is observed to quickly recover.

The radius dynamics of two bubbles belonging to this particular succession are compared with simulations in Figure 4.15. The agreement is quite good once the (a priori unknown) initial supersaturation level $\zeta$ is adjusted. Note that $\zeta$ corresponds to the mean value of the initial concentration profile close to the electrode wall. As before, $\zeta$, or rather the initial concentration profile, is computed from (4.17), and it is a function of the current density and time of nucleation after the onset of electrolysis. The value of the effective current density employed is $1.04 \mathrm{~A} / \mathrm{m}^{2}$ : the same as the one obtained by fitting the initial growth rate of the third bubble in the succession (as done for Figure 4.13a). Thus, the nucleation time after the onset of electrolysis is now the sole free parameter. Setting it equal to the experimental nucleation time clearly overestimates the growth rates, as seen from the zero-loss prediction curves in Figure 4.15. One can then account for depletion losses by decreasing the time of nucleation used to calculate $\zeta$. After a suitable reduction in the nucleation time, and hence $\zeta$, the bubble growth behaviour is reasonably well reproduced.

One may note that in the supersaturation regime, the zero-concentration boundary condition is not required since the entire cavity, and possibly a small region above it, will be homogeneously supersaturated to a good degree. As the bubble approaches departure, a retardation in its growth is noticeable nonetheless. This is attributed to $\mathrm{H}_{2}$ deprivation due to the presence of parasitic bubbles. The deviation for the $48^{\text {th }}$ bubble is greater than for the $74^{\text {th }}$ bubble. This is consistent with the fact that the $48^{\text {th }}$ bubble is deprived from the $\mathrm{H}_{2}$ available to a greater extent by the aforementioned large parasitic bubble. 


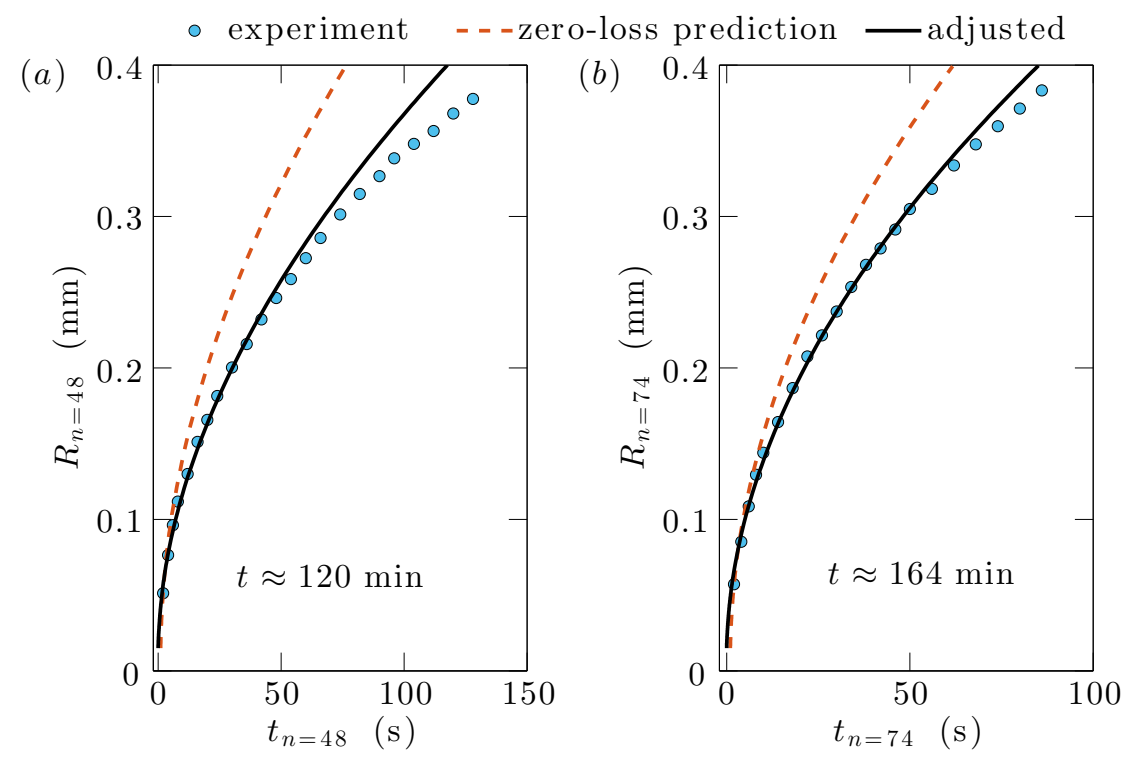

Figure 4.15: Comparison between simulation (curves) and the experimental growth dynamics for the (a) $48^{t h}$ and (b) $74^{\text {th }}$ bubble of the succession with $H_{p}=30 \mu \mathrm{m}$ (cf. Figure 4.14). The nominal current density is $I_{w}=7.8 \mathrm{~A} / \mathrm{m}^{2}$, whereas simulations employ an effective current density of $1.04 \mathrm{~A} / \mathrm{m}^{2}$. The red dashed curves are expected growth for a bubble in an infinitely long electrode that nucleates at the experimental time $t$ in the absence of any previous bubbles. The initial concentration profile is given by (4.17) evaluated at time $t$. The resulting local supersaturation is $\zeta_{48} \approx 9.25$ and $\zeta_{74} \approx 11.0$. For the black curves the time is adjusted to $(a)$ $0.5 t$ and $(b) 0.6 t$ resulting in $\zeta_{48} \approx 7.25$ and $\zeta_{74} \approx 8.4$. 


\subsection{Conclusions}

The growth dynamics of a succession of $\mathrm{H}_{2}$ bubbles driven by constant-current electrolysis on a large electrode has been studied. The bubbles form within a hydrophobic pit on top of a single micropillar etched on a flat silicon electrode. The large surface area of the electrode ensures that the bubble growth is diffusion-limited. As such, growth can be suitably described in terms of a (experimentally-determined) dimensionless growth coefficient $\tilde{\beta}_{\mathrm{H}_{2}}$ according to $R(t)=\tilde{\beta}_{\mathrm{H}_{2}} \sqrt{D_{\mathrm{H}_{2}} t}$.

The accumulation of dissolved $\mathrm{H}_{2}$ near the gas-evolving electrode is hindered by several depletion sources. We identify depletion from (i) previous bubbles in the succession, from (ii) unwanted parasitic bubbles forming on the cavity sidewall, and from (iii) the mere presence of the cavity where the electrode is being held. It follows that the degree of supersaturation near the electrode (and consequently $\tilde{\beta}_{H_{2}}$ ) is largely unsteady. For the range of current densities considered in our experiments (5-15 A/ $\mathrm{m}^{2}$ ), the $\mathrm{H}_{2}$ bubble succession first experiences a stagnation regime where depletion effects are most noticeable. Here, $\tilde{\beta}_{H_{2}}$ remains fairly constant or decreases. A fast growth in $\tilde{\beta}_{H_{2}}$ then follows before the steady state is reached. Conversely, for the case of $\mathrm{CO}_{2}$ bubbles in our P-C experiments, persistent depletion (exclusively from previous bubbles in the succession) is observed. The analogous growth coefficient $\tilde{\beta}_{\mathrm{CO}_{2}}$ decreases in a way such that the quantity $1 / \tilde{\beta}_{C O_{2}}^{2}(t)$ increases linearly with time.

Furthermore, in the electrolysis experiments, the depletion effect of the cavity opening and of parasitic bubbles forming on the cavity sidewall account for the growth retardation observed as bubbles approach their departure size. Oppositely, $\mathrm{CO}_{2}$ bubbles experience enhanced growth as they approach detachment. This is attributed to the onset of density-induced natural convection.

The comparison of both case scenarios serves to highlight the important effect of depletion, omnipresent in the growth of a succession of bubbles. The knowledge obtained can be further expanded by conducting electrolysis experiments with two main variations. Firstly, we have nucleated bubbles within a 'cavity' with no flow (stagnant conditions). In real-life scenarios, however, it may be desirable to have continuous flow. This will change the force balance keeping the bubbles pinned at the surface, and influence mass transport through advection. Secondly, closed systems that could be pressurised (as opposed to open systems, such as the one presented here) will be much similar to the actual utilisation of microfluidic electrochemical reactors [46]. Both cases are expected to entail distinct bubble growth dynamics, and certainly warrant further study. 


\section{Appendix A. 1D concentration profile from a gas-evolving surface}

The one-dimensional diffusion equation governing the evolution of the dissolved gas concentration profile

$$
\frac{\partial C}{\partial t}=D \frac{\partial^{2} C}{\partial z^{2}}
$$

applies to the the liquid region $0<z<H$. A constant molar flux $J_{w}$ enters the region at $z=0$. At the free surface, $C(z=H, t)=0$. Initially we assume that $C(z, t=0)=0$. The concentration profile has the following analytical solution [190]:

$C(z, t)=2 J_{w} \sqrt{t / D} \sum_{n=0}^{\infty}(-1)^{n}\left\{\operatorname{ierfc}\left(\frac{n H+z / 2}{\sqrt{D t}}\right)-\operatorname{ierfc}\left(\frac{(n+1) H-z / 2}{\sqrt{D t}}\right)\right\}$

where the integral of the complementary error function is defined as

$$
\operatorname{ierfc}(x)=\int_{x}^{\infty} \operatorname{erfc}(u) \mathrm{d} u=\frac{\mathrm{e}^{-x^{2}}}{\sqrt{\pi}}-x \operatorname{erfc}(x) .
$$

Equation (4.17) converges in time to the linear profile of the steady state solution, $C(z, t \rightarrow \infty)=J_{w}(H-z) / D$. Profiles computed from (4.17) are plotted in Figure 4.16, whereas the evolution in time of the concentration at the gas-evolving surface $(z=0)$ is plotted in Figure 4.17. Note that for the case of infinite height, $H \rightarrow \infty$, the solution in (4.17) simplifies to

$$
C(z, t)=2 J_{w} \sqrt{t / D} \operatorname{ierfc}\left(\frac{z}{\sqrt{4 D t}}\right) .
$$

In this particular case, no steady-state solution exists, and the concentration at the wall increases unboundedly as $2 J_{w} \sqrt{t /(\pi D)}$. Furthermore, if small heights or short times are considered such that $z / \sqrt{4 D t} \ll 1$, then (4.19) can be approximated as

$$
C(z, t)=\frac{2 J_{w}}{\sqrt{\pi D}}\left(\sqrt{t}-\frac{z}{2} \sqrt{\pi / D}\right)
$$




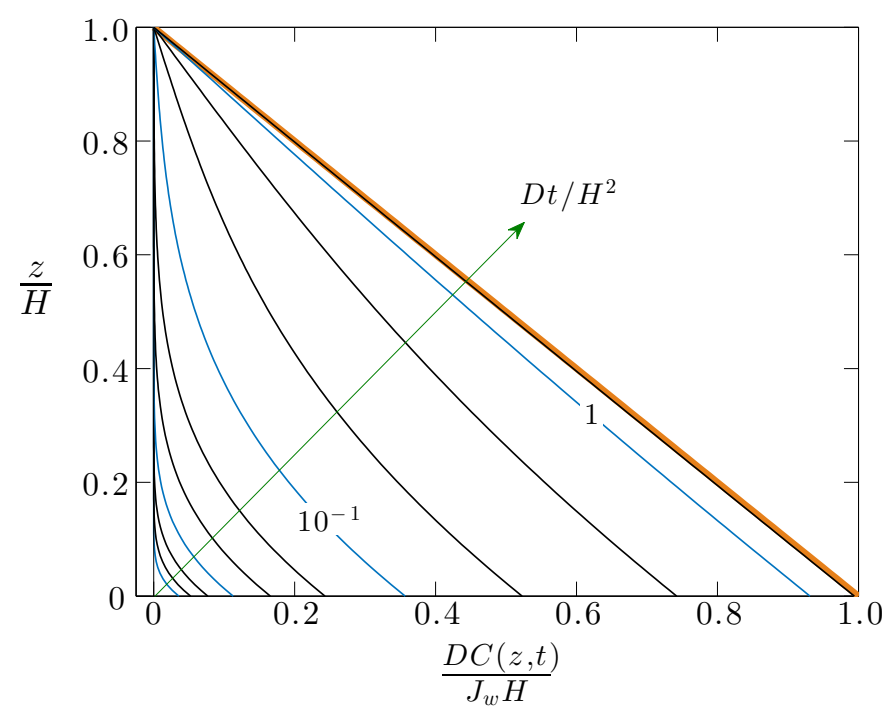

Figure 4.16: Universal plot of the time evolution of the 1D concentration profiles for different instants in dimensionless time. The profiles marked in blue correspond to $\log _{10}\left(D t / H^{2}\right)=-3,-2,-1$ and 0 . The profile in red is the steady-state profile.

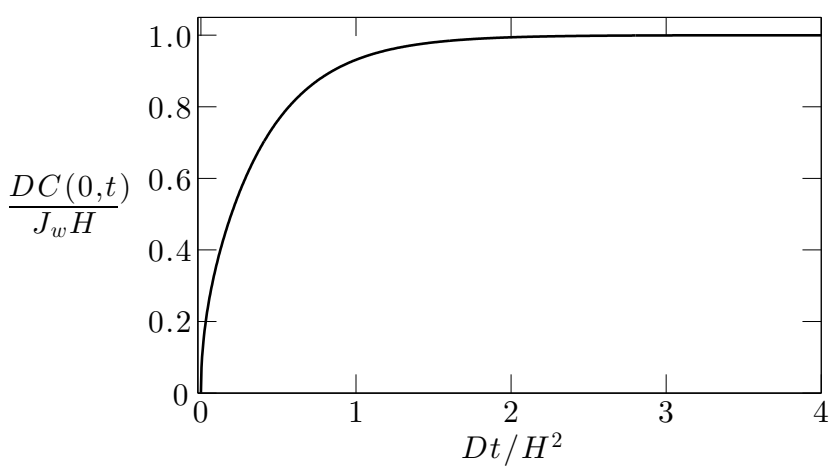

Figure 4.17: Invariant, dimensionless plot of the time evolution of the concentration at the electrode wall $(z=0)$. 


\section{Appendix B. Depletion model for the growth coefficient}

Let subscript $n$ refer to the $n^{\text {th }}$ bubble. Let $t_{n}=t-t_{0, n}$, where $t_{0, n}$ is the nucleation time of such bubble and $t_{0, n=1}=0$. Let $\theta_{n}(r, t)=C(r, t)-\bar{C}_{n}$, where

$$
\bar{C}_{n}=C_{n}\left(r, t_{n}=0\right)=C_{n-1}\left(r=\delta_{n-1}, t_{n-1}=\Delta t_{n-1}\right)
$$

is the characteristic (homogeneous) concentration field (left behind by bubble $n-1$ ) in which bubble $n$ immediately grows. It is essentially the concentration field evaluated at the characteristic diffusion length $\delta_{n}$ and time $\Delta t_{n}$ corresponding to the previous bubble, where $\Delta t_{n}=t_{d, n}-t_{0, n}$ is the bubble residence time. Note that we assume negligible time between detachment and nucleation of the subsequent bubble: $t_{n-1}=\Delta t_{n-1}$ is equivalent to $t_{n}=0$. Furthermore, the detachment radius $R_{d}=\tilde{b}_{n} \sqrt{D \Delta t_{n}}$ is assumed constant for all bubbles.

Following the model proposed by Moreno Soto et al. (2017)[103], we treat each bubble in the succession as a point source of molar strength

$$
\dot{n}_{n}=-\frac{4 \pi P_{0} R_{d}^{3}}{3 R_{u} T_{\infty} \Delta t_{n}}
$$

which acts as a boundary condition to the diffusion equation,

$$
\frac{\partial \theta_{n}}{\partial t_{n}}=\frac{D}{r^{2}} \frac{\partial}{\partial r}\left(r^{2} \frac{\partial \theta_{n}}{\partial r}\right)
$$

together with a second boundary condition $\theta_{n}(r \rightarrow \infty, t)=0$ and initial condition $\theta_{n}\left(r, t_{n}=0\right)=0$. Let $\bar{\theta}_{n}$ be the characteristic solution for $\theta_{n}$ evaluated at $r=\delta_{n}$ and $t=\Delta t_{n}$. Then,

$$
\bar{\theta}_{n}=-\frac{4 \pi P_{0} R_{d}}{3 R_{u} T_{\infty}} \frac{\tilde{b}_{n}^{2}}{\delta_{n}} \operatorname{erfc}\left(\frac{\delta_{n} \tilde{b}_{n}}{2 R_{d}}\right) .
$$

Let $\Delta \bar{C}_{n}=\bar{C}_{n}-k_{H} P_{0}$ be the characteristic concentration field strength relative to the interfacial concentration. One then has that

$$
\Delta \bar{C}_{n}=\bar{C}_{1}-k_{H} P_{0}+\sum_{m=2}^{n} \bar{\theta}_{m} .
$$

Making use of (4.9), we know that $\Delta \bar{C}_{n}=P_{0} \tilde{b}_{n}^{2} /\left(2 R_{u} T_{\infty}\right)$. Substituting this expression along with (4.24) into (4.25) yields

$$
\tilde{b}_{n}^{2}=\tilde{b}_{1}^{2}-\frac{8 \pi}{3} \sum_{m=2}^{n} R_{d} \frac{\tilde{b}_{m}^{2}}{\delta_{m}} \operatorname{erfc}\left(\frac{\delta_{m} \tilde{b}_{m}}{2 R_{d}}\right),
$$


where $\tilde{b}_{1}^{2}=2 R_{u} T_{\infty} \Delta \bar{C}_{1} / P_{0}$ is just the known growth rate of the first bubble (corresponding to the initial, undepleted supersaturation $\Delta \bar{C}_{1}$ ). From experimental evidence in Moreno Soto et al. (2017)[103], we can make the approximation that $\delta_{n} \approx 4 R_{d}$. Moreover, since $\tilde{b}_{m} \sim 0.1$ is small, the complementary error function can be approximated as unity. Equation (4.26) then simplifies to

$$
\tilde{b}_{n}^{2}=\tilde{b}_{1}^{2}-\frac{2 \pi}{3} \sum_{m=2}^{n} \tilde{b}_{m}^{2} .
$$

The above expression can be made continuous in time through

$$
\tilde{b}^{2}(t)=\tilde{b}^{2}(0)-\frac{2 \pi}{3} \int_{0}^{t} \tilde{b}^{2}\left(t^{\prime}\right) \frac{\mathrm{d} n}{\mathrm{~d} t^{\prime}} \mathrm{d} t^{\prime}
$$

where $\mathrm{d} n / \mathrm{d} t=D \tilde{b}^{2}(t) / R_{d}^{2}$ is simply the continuous form of the bubble nucleation frequency. Differentiating (4.28) in time yields

$$
2 \tilde{b} \frac{\mathrm{d} \tilde{b}}{\mathrm{~d} t}=-\frac{2 \pi D}{3 R_{d}^{2}} \tilde{b}^{4} .
$$

Upon integration, we obtain

$$
\frac{1}{\tilde{b}^{2}(t)}-\frac{1}{\tilde{b}^{2}(0)}=\frac{2 \pi}{3} \frac{D t}{R_{d}^{2}} .
$$

\section{Acknowledgement}

The authors thank S. Schlautmann for his fruitful contribution to this work. This work was supported by the Netherlands Centre for Multiscale Catalytic Energy Conversion (MCEC), an NWO Gravitation programme funded by the Ministry of Education, Culture and Science of the government of the Netherlands. We also acknowledge the support of the Spanish Ministry of Economy and Competitiveness through grants DPI2014-59292-C3-1-P and DPI2015-71901-REDT, partly funded through European Funds. 


\section{Supporting information}

This supporting information provides the mathematical formulation of the mass transfer problem in addition to the equations employed by the numerical model. In particular we focus on $(a)$ the electrolytic growth of a single $\mathrm{H}_{2}$ bubble attached to a micropillar protruding from a $\mathrm{H}_{2}$ gas-evolving substrate in an air-saturated, $\mathrm{H}_{2}$-containing, electrolyte and (b) the growth of a $\mathrm{CO}_{2}$ bubble attached to a micropillar in mildly supersaturated carbonated water.

\section{Formulation of the mass transfer problem}

The transport of dissolved gas species in liquids is governed by the mass transport equation, usually referred to as the advection-diffusion equation. The bubbles evolving in the electrolysis experiments are immersed in a binary system of dissolved hydrogen gas and air. We choose to model air as a single species. The advection-diffusion equation for each gas species reads

$$
\frac{\partial C_{k}}{\partial t}+\boldsymbol{U} \cdot \nabla C_{k}=D_{k} \nabla^{2} C_{k}, \quad k=1,2
$$

where $C_{k}(\boldsymbol{x}, t)$ is the molar-based concentration field of dissolved hydrogen gas $(k=1)$ or air $(k=2)$. The concentration field and the velocity vector field $\boldsymbol{U}(\boldsymbol{x}, t)$ are both functions of the position vector $\boldsymbol{x}$ and time $t$. The gas diffusivities are denoted by $D_{k}$.

The pressure-controlled (P-C) experiments obviously involve monocomponent $\mathrm{CO}_{2}$ bubbles. In this case, the problem is simplified to a single gas species. Therefore, $k=1$ in (4.31) refers to $\mathrm{CO}_{2}$ gas, whereas $k=2$ is not applicable. From hereon forth, the problem shall be formulated for the electrolysis problem. The P-C problem is identical, except that the molar flux of $\mathrm{CO}_{2}$ at the substrate is zero $\left(J_{w}=0\right)$, and species $k=2$ should be removed from the formulation. The initial conditions are somewhat different. These shall be pointed out correspondingly.

The $\mathrm{H}_{2}$ gas generated at the gas-evolving electrode can be suitably described through a constant flux boundary condition at the electrode surface,

$$
\nabla C_{1} \cdot \hat{\boldsymbol{n}}=-J_{w} / D_{1}
$$

where $\hat{\boldsymbol{n}}$ is the outward-pointing unit normal from the electrode surface. One would expect a zero-flux boundary condition along the impermeable holder and cavity walls, whereas at the free surface a zero-concentration boundary condition applies. For air, we expect a zero-flux boundary condition along the walls of the substrate and holder. At the free surface, the electrolyte is perfectly saturated with air. The boundary condition for 
the dissolved gas concentration at the bubble surface, $C_{k, i}(t)$, is given by Henry's Law,

$$
C_{k, i}(t)=k_{H, k} \frac{n_{k}}{n}\left(P_{0}+\frac{2 \gamma_{l g}}{R}\right),
$$

where $n_{k}(t)$ is the number of moles of species $k$ inside the bubble, $n(t)=$ $n_{1}(t)+n_{2}(t)$ is the total number of moles, $P_{0}$ is the ambient pressure, $\gamma_{l g}$ is the liquid-gas surface tension and $R(t)$ is the bubble radius.

The bubble gas volume is related to the gas content and pressure via the equation of state for an ideal gas,

$$
\frac{4}{3} \pi R^{3}\left(P_{0}+\frac{2 \gamma_{l g}}{R}\right)=n R_{u} T_{\infty}
$$

where $R_{u}$ denotes the universal gas constant and $T_{\infty}$ is the ambient temperature. Finally, the mass transfer rate across the bubble surface $S$ is given by Fick's first law. The molar rate of gas within the bubble is thus

$$
\frac{\mathrm{d} n}{\mathrm{~d} t}=\sum_{k=1}^{2} \frac{\mathrm{d} n_{k}}{\mathrm{~d} t}=\sum_{k=1}^{2} D_{k} \int_{S} \boldsymbol{\nabla} C_{k} \cdot \hat{\boldsymbol{n}} \mathrm{d} S
$$

where $\mathrm{d} S$ is an infinitesimal area element of the bubble surface, and here $\hat{\boldsymbol{n}}$ is the outward-pointing unit normal from the bubble surface.

\section{Nondimensionalization}

It is convenient to rewrite the time, radius, Cartesian coordinates, mole number and concentration in dimensionless form:

$$
\begin{array}{r}
\tau=\frac{D_{1}}{R_{0}^{2}} t, \quad a=\frac{R(t)}{R_{0}}, \quad \tilde{\boldsymbol{x}}=\frac{\boldsymbol{x}}{R_{0}}, \\
\mu_{k}=\frac{R_{u} T_{\infty}}{4 / 3 \pi R_{0}^{3} P_{0}} n_{k}(t), \quad c_{k}=\frac{C_{k}(\boldsymbol{x}, t)-C_{k, \infty}}{k_{H, k} P_{0}} .
\end{array}
$$

We also normalise the pillar height $h_{p}=H_{p} / R_{0}$, pillar radius $r_{p}=R_{p} / R_{0}$ and the free surface height $h=H / R_{0}$ using the pit radius $R_{0}$. The reference concentrations are $C_{1, \infty}=0$ for $\mathrm{H}_{2}$ and $C_{2, \infty}=k_{H, 2} P_{0}$ for air. Moreover, the species diffusivity, reference concentration and solubility, in addition to the surface tension and $\mathrm{H}_{2}$ flux, may all be written in dimensionless form,

$$
\begin{gathered}
\Gamma_{k}=\frac{D_{k}}{D_{1}}, \quad \Upsilon_{k}=\frac{C_{k, \infty}}{k_{H, k} P_{0}}, \quad \Lambda_{k}=k_{H, k} R_{u} T_{\infty}, \\
\sigma=\frac{2 \gamma_{l g}}{R_{0} P_{0}}, \quad j_{w}=\frac{R_{0} J_{w}}{D_{1} k_{H, 1} P_{0}} .
\end{gathered}
$$

One may note that $\Upsilon_{1}=0$ whereas $\Upsilon_{2}=1$. For the P-C problem, $\Upsilon_{1}=P_{s} / P_{0}$ (approximately 1.17 in the experiments), where $P_{s}$ denotes the saturation pressure. 


\section{Simulation overview}

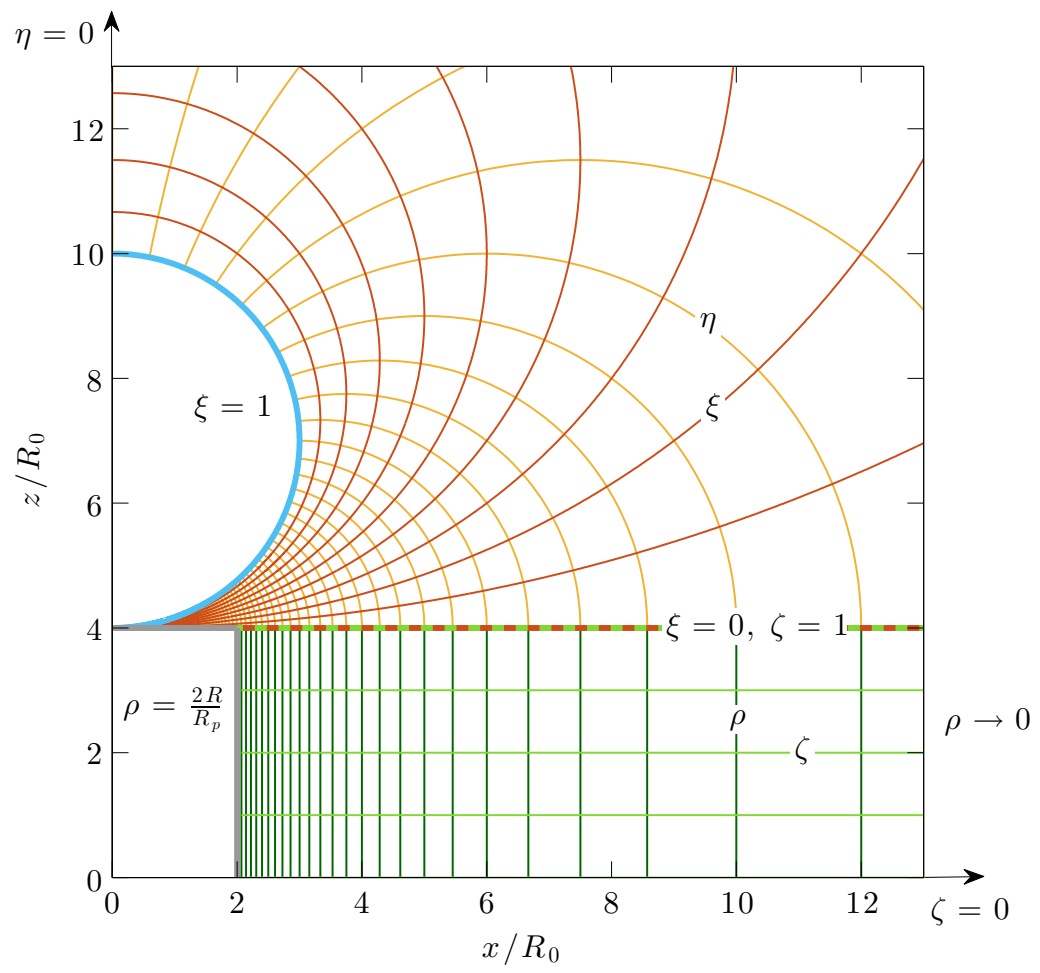

Figure 4.18: Schematic of the simulation grid. The simulation points fall on the intersection of the contourlines. The simulation is axisymmetric around the $z$-axis. Drawn for $H_{p} / R_{0}=4$ and $R_{p} / R_{0}=2$. The bulk region in tangent-sphere coordinates $(\eta, \xi)$ applies to $z>H_{p}$. The microlayer region in pseudo-cylindrical coordinates $(\rho, \zeta)$ applies to $0<z<H_{p}$. A matching condition is imposed at $z=H_{p}$. Coordinates $\eta$, $\xi$ and $\rho$ evolve with the bubble, so that $\xi=1$ (in blue) always maps the bubble surface, regardless of the actual bubble size. Here, the bubble size has been arbitrarily set to $R / R_{0}=3$. The separation of the contours is uniform. Here, $\Delta \eta=\Delta \xi=$ $\Delta \rho=0.1$ and $\zeta=0.5$. The coordinate $\eta=0$ lies on the $z$-axis, $\eta \rightarrow \infty$ at the contact point.

As discussed in the main manuscript, neither the geometry of the holder and cavity, nor the free surface, is directly modelled. The simulated scenario is simplified to that of a bubble growing on a pillar upon an a substrate of infinite area in a semi-infinite liquid. The simulation solves the mass transfer problem for this simplified configuration. The computational grid, sketched in Figure 1, is divided into two regions that use different 
coordinate systems: a bulk region for $z>H_{p}$ and a microlayer region below. In the bulk region, the advection-diffusion equation (1) coupled with streamfunction-vorticity equations are solved imposing a no-slip boundary condition at $z=H_{p}$. In the microlayer region, only the diffusion equation is solved, taking into account the flux boundary condition (2) across the substrate wall and the pillar surface. The concentration and its gradient are matched at the coupling plane $z=H_{p}$.

Overall, the numerical model is an extension of the (pillar-less) numerical model described in Peñas-López et al (2017)[187]. A detailed description of the numerical model and procedure may be found there. The novelties here are $(a)$ the consideration of multiple species, $(b)$ the addition of the microlayer region (as a means to overcome the geometrical difficulty imposed by the pillar) and $(c)$ the non-zero $\mathrm{H}_{2}$ flux boundary condition at the substrate. These additions or changes strictly affect only the mass transfer equations or their boundary conditions. The streamfunction-vorticity formulation and numerical procedure remains unchanged. For this reason, expressions for just these particular mass transfer equations shall be provided next.

\section{Equations for the bulk region}

The mass transfer problem is recast in dimensionless tangent-sphere spatial coordinates $(\eta, \xi, \phi)$, where

$$
\tilde{x}=2 a \frac{\eta}{\eta^{2}+\xi^{2}} \cos \phi, \quad \tilde{y}=2 a \frac{\eta}{\eta^{2}+\xi^{2}} \sin \phi, \quad \tilde{z}=2 a \frac{\xi}{\eta^{2}+\xi^{2}}+h_{p} .
$$

The scale factors

$$
\tilde{h}(\eta, \xi)=\tilde{h}_{\eta}=\tilde{h}_{\xi}=\frac{2 a}{\eta^{2}+\xi^{2}}, \quad \tilde{h}_{\phi}(\eta, \xi)=\frac{2 a \eta}{\eta^{2}+\xi^{2}} .
$$

also depend on the instantaneous bubble radius $a(\tau)$. The dimensionless advection-diffusion equation for $c_{k}(\tilde{\boldsymbol{x}}, \tau)$ reads

$$
\frac{\partial c_{k}}{\partial \tau}+\boldsymbol{u} \cdot \tilde{\nabla} c_{k}=\tilde{\nabla}^{2} c_{k}
$$

and, for $c_{k}(\eta, \xi, \tau)$, it transforms to

$$
\frac{\partial c_{k}}{\partial \tau}=F \frac{\partial c_{k}}{\partial \eta}+G \frac{\partial c_{k}}{\partial \xi}+H\left(\frac{\partial^{2} c_{k}}{\partial \eta^{2}}+\frac{\partial^{2} c_{k}}{\partial \xi^{2}}\right)
$$

where

$$
F=-\Gamma_{k} \frac{\eta^{2}-\xi^{2}}{2 a \tilde{h} \eta}-\frac{\mathrm{d} a}{\mathrm{~d} \tau} \frac{\eta}{a}-\frac{u_{\eta}}{\tilde{h}}, \quad G=-\Gamma_{k} \frac{\xi}{a \tilde{h}}-\frac{\mathrm{d} a}{\mathrm{~d} \tau} \frac{\xi}{a}-\frac{u_{\xi}}{\tilde{h}}, \quad H=\frac{\Gamma_{k}}{\tilde{h}^{2}} .
$$


The velocity field components $u_{\eta}(\eta, \xi, \tau)$ and $u_{\xi}(\eta, \xi, \tau)$ are computed as detailed in Peñas-López et al[187]. The boundary conditions for the concentration at the bubble surface, axis of symmetry and triple contact point are

$$
c_{k}(\eta, 1, \tau)=\frac{\mu_{k}}{\mu}\left(1+\frac{\sigma}{a}\right)-\Upsilon_{k}, \quad \frac{\partial c_{k}}{\partial \eta}(0, \xi, \tau)=0, \quad \frac{\partial c_{k}}{\partial \eta}(\infty, \xi, \tau)=0 .
$$

At the coupling plane, we prescribe the flux $j_{w, k}$ at the pillar top surface, and we force a coupling flux $j_{c p, k}(\eta)$ in the liquid phase equal to the flux obtained from the microlayer region:

$$
\frac{\partial c_{k}}{\partial \xi}(\eta, 0, \tau)= \begin{cases}-\tilde{h}(\eta, 0) j_{w, k}, & \text { if } \eta>2 a / r_{p} \\ -\tilde{h}(\eta, 0) j_{c p, k}(\eta), & \text { otherwise. }\end{cases}
$$

The dimensionless molar flow rate across the bubble surface is

$$
\frac{\mathrm{d} \mu}{\mathrm{d} \tau}=-\left.3 a \sum_{k=1}^{2} \Gamma_{k} \Lambda_{k} \int_{0}^{\infty} \frac{\eta}{1+\eta^{2}} \frac{\partial c_{k}}{\partial \xi}\right|_{\xi=1} \mathrm{~d} \eta .
$$

Finally, the dimensionless ideal gas law simply reads: $(1+\sigma / a) a^{3}=\mu$.

\section{Diffusion equation for the microlayer region}

In the microlayer region bounded by $\tilde{x}^{2}+\tilde{y}^{2}>r_{p}^{2}$ and $0 \leq \tilde{z} \leq h_{p}$, the diffusion equation is recast in dimensionless pseudo-cylindrical coordinates $(\rho, \phi, \zeta)$,

$$
\tilde{x}=\frac{2 a}{\rho} \cos \phi, \quad \tilde{y}=\frac{2 a}{\rho} \sin \phi, \quad \tilde{z}=h_{p} \zeta .
$$

The scale factors are

$$
\tilde{h}_{\rho}=\frac{2 a}{\rho^{2}}, \quad \tilde{h}_{\phi}=\frac{2 a}{\rho}, \quad \tilde{h}_{\zeta}=h_{p} .
$$

One may note that coordinate $\rho$ behaves identically to $\eta$ in the coupling plane defined by $\left(\rho, \phi, \tilde{z}=h_{p}\right)$ or $(\eta, \xi=0, \phi)$. The microlayer region is bounded by $0<\rho \leq \rho_{p}(\tau)$ and $0 \leq \zeta \leq 1$ where $\rho_{p}=2 a / r_{p}$. The diffusion equation for $c_{k}(\rho, \zeta, \tau)$ then reads

$$
\frac{\partial c_{k}}{\partial \tau}=A \frac{\partial c_{k}}{\partial \rho}+B \frac{\partial^{2} c_{k}}{\partial \rho^{2}}+C \frac{\partial^{2} c_{k}}{\partial \zeta^{2}}
$$

where

$$
A=\frac{\Gamma_{k}}{\rho \tilde{h}_{\rho}^{2}}-\frac{\mathrm{d} a}{\mathrm{~d} \tau} \frac{\rho}{a}, \quad B=\frac{\Gamma_{k}}{\tilde{h}_{\rho}^{2}}, \quad C=\frac{\Gamma_{k}}{\tilde{h}_{\zeta}^{2}}
$$


The boundary conditions are

$$
\frac{\partial c_{k}}{\partial \rho}(0, \zeta, \tau)=0, \quad \frac{\partial c_{k}}{\partial \rho}\left(\rho_{p}, \zeta, \tau\right)=2 a j_{w, k} / \rho_{p}^{2}, \quad \frac{\partial c_{k}}{\partial \zeta}(\rho, 0, \tau)=-h_{p}^{2} j_{w, k} .
$$

At the coupling plane, we impose the concentration found at the coupling plane in the bulk region:

$$
c_{k}(\rho, \zeta=1, \tau)=c_{k}(\eta, \xi=0, \tau) .
$$

The flux obtained the coupling plane of the microlayer region is then imposed as the flux boundary condition for the bulk region at the coupling plane,

$$
-j_{c p, k}(\eta, \tau)=\frac{1}{\tilde{h}(\eta, 0)} \frac{\partial c_{k}}{\partial \xi}(\eta, 0, \tau)=\frac{1}{h_{p}} \frac{\partial c_{k}}{\partial \zeta}(\rho, 1, \tau) .
$$

\section{Initial conditions of the numerical model}

Let us consider a bubble that grows at time $\tau=\tau_{0}$ after the start of electrolysis. The initial condition for the concentration of air is assumed always to be $c_{2}\left(\eta, \xi, \tau_{0}\right)=0$, i.e. the electrolyte is uniformly equilibrated with air. Furthermore, we approximate the initial $\mathrm{H}_{2}$ concentration field as the 1D vertical profile that would be obtained in the total absence of previous bubbles (cf. Appendix A in the manuscript):

$$
\begin{aligned}
& c_{1}\left(\eta, \xi, \tau_{0}\right)= \\
& 2 j_{w} \sqrt{\tau_{0}} \sum_{n=0}^{\infty}(-1)^{n}\left\{\operatorname{ierfc}\left(\frac{n h+\tilde{z}_{i} / 2}{\sqrt{\tau_{0}}}\right)-\operatorname{ierfc}\left(\frac{(n+1) h-\tilde{z}_{i} / 2}{\sqrt{\tau_{0}}}\right)\right\} .
\end{aligned}
$$

where $\tilde{z}_{i}=2 \xi a_{i} /\left(\eta^{2}+\xi^{2}\right)+h_{p}$. Here, $a_{i}=a\left(\tau_{0}\right)$ is the initial bubble radius which must be set to a small but finite size (typically around two times the pit radius). The initial bubble composition, $\mu_{1}\left(\tau_{0}\right)$ and $\mu_{2}\left(\tau_{0}\right)$ (whether purely $\mathrm{H}_{2}$, purely air, or a mixture of both), was seen to have a negligible effect on the bubble growth dynamics

The initial concentration condition for the P-C problem is that the water is homogeneously supersaturated with $\mathrm{CO}_{2}, c_{1}\left(\eta, \xi, \tau_{0}\right)=0$. Moreover, the initial time can be set to $\tau_{0}=0$ since its value is of no consequence. 


\section{Electronic supplementary information}

The following QR codes can be scanned to access the electronic supplementary information.

The QR code in Figure 4.19 leads to the electronic supplementary information file. The file is a video of successive evolving bubbles generated with a current density of $10.4 \mathrm{~A} / \mathrm{m}^{2}$. The bubble radius and dimensionless growth coefficient are plotted against the experimental time.

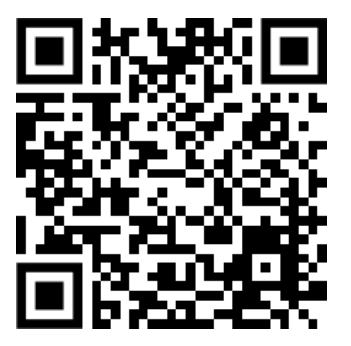

Figure 4.19: Movie of a particular electrolysis experiment.

The following url will lead to this video as well: http: //pubs.acs.org/doi/suppl/10.1021/acs. langmuir.7b02978/suppl_ file/la7b02978_si_002.avi. 


\section{Spatial control over electrolytic bubbles nearby gas evolving electrodes}

Evolving gas bubbles hinder water-electrolysis and therefore their role in electrolysis must be fundamentally understood. We show that the dynamics of the growing bubbles on the electrodes leads to fluctuations in the electrode potential. To reduce this problem, we present a structured substrate where bubbles grow nearby the gas-evolving electrodes. Water was electrolyzed with circular ring electrodes surrounding superhydrophobic cavities, on which hydrogen bubbles formed. The electrical potential drops when bubbles detach, due to convection in the liquid disrupting the dissolved gas boundary layer. This disruption causes a fluctuation in the concentration overpotential, with voltage drops positively correlated with the detachment volume of the bubbles. 


\subsection{Introduction}

Electrochemical manufacturing is responsible for the production of important large-volume products such as sodium, hydroxide, chlorine, aluminum and hydrogen [191, 192, 25]. All of these processes involve multiphase electrolytes, as gasses evolve from at least one of the electrodes of their respective electrochemical cells. The bubbles that are formed often block active electrocatalyst sites, and lower the energy conversion efficiency of the electrochemical reactions of interest $[56,126]$.

Bubble nucleation in electrolysis occurs when the saturation threshold of dissolved gases in an electrolyte is reached. The driving force of the nucleation process is the chemical potential which increases with gas concentration [78]. Therefore, nucleation occurs typically on the gas evolving electrodes not only because heterogeneous nucleation has a lower thermodynamic energy barrier than homogeneous nucleation [82, 81, 83, $13]$, but also because at gas evolving electrodes the gas concentration is larger than in the bulk liquid.

The critical radius, the minimum nucleus size that must be reached before a gas nucleus is stable and begins to grow, has been defined as $[193,194,195,153]$ :

$$
R_{c}=\frac{2 \sigma}{P_{s} \zeta}
$$

with $\sigma$ the surface tension, $P_{s}$ the pressure of the bulk liquid, and $\zeta$ the supersaturation level. The critical radius is slightly lowered by a reduced surface tension of the electrolyte, which can occur by dissolved gases and electric fields [120, 121, 85]. It has been demonstrated that shape [78] and surface roughness of substrates [85] play an important role on the nucleation barrier as well. For example, a flat electrode has a larger nucleation barrier than an electrode with an acute or obtuse angle [81, 83]. Similarly, a smooth electrode has a larger nucleation barrier than a rough electrode [196] due the possibility of pre-existing nuclei, originating from trapped air, within the crevices of the roughness [195].

Superhydrophobic cavities have been used recently in studies on water electrolysis $[28,27]$. Upon submersion of these superhydrophobic cavities in liquid, an air pocket is trapped within the cavity. The air pocket has a meniscus and when the critical radius is smaller than the radius of curvature of this meniscus, for example by the increase in supersaturation level, the meniscus expands outwards and a bubble is formed. The bubble formation on such cavities is achieved at supersaturation levels lower than those for a surrounding substrate.

Since the presence of bubbles can cause losses in electrochemical processes, we study the electrical response to electrolytic bubble evolution 
on the cavity which is the nucleation, growth, and detachment of bubbles during the electrolysis of water. Because bubble evolution on an electrode leads to potential fluctuations, this study explores a new concept to separate bubble nucleation from the electrode by introducing weak spots far away from the electrode, in order to promote nucleation at that location, rather than on the electrode itself. We do so by superhydrophibic cavities micromachined in the center of a flat platinum electrode ring. In this way the energy landscape for nucleation can be in favor of the cavity, and not on the electrode surface, resulting in bubble generation on the cavity and away from the electrode and reduced potential fluctuations. The chapter is organized as follows: the experimental section reports on the novel electrode design and equipment used in this work, the results and discussion of the measurements follows. Finally, a summary of the work is provided in which an outlook is given.

\subsection{Experimental}

The novel electrode design consists of a platinum ring, with a $400 \mu \mathrm{m}$ inner radius (width varied between $10 \mu \mathrm{m}, 25 \mu \mathrm{m}$, and $50 \mu \mathrm{m}$ ) around a superhydrophobic cavity (radii were varied between $5 \mu \mathrm{m}, 10 \mu \mathrm{m}, 15 \mu \mathrm{m}$, and $25 \mu \mathrm{m}$ ) etched into silicon substrates. The substrates were glued in glass containers of $20 \times 60 \times 20 \mathrm{~mm}^{3}$, later filled with an electrolyte with different pH levels; pH 1 (0.2 M KCl, pH adjusted with $\mathrm{HCl}), \mathrm{pH} 3(0.1 \mathrm{M}$ $\mathrm{C}_{8} \mathrm{H}_{5} \mathrm{KO}_{4}, \mathrm{pH}$ adjusted with $\left.\mathrm{HCl}\right), \mathrm{pH} 7$ and $\mathrm{pH} 10\left(0.05 \mathrm{M} \mathrm{NaHCO}_{3}, \mathrm{pH}\right.$ adjusted with $\mathrm{NaOH}$ ). A schematic overview of the setup is shown in Figure 5.1A.

A two-electrode setup was used to reduce probable nucleation of bubbles on the surface of a reference electrode. We define bubble nucleation as the onset of bubble growth, which in our experimental work starts when a bubble radius reaches $25 \mu \mathrm{m}$ so that we obtain a clear image contrast also when bubbles expand from the largest cavities. Optical microscopy and fast cameras allowed capturing sequences of bubble growth and detachment. More details on the fabrication and setup can be found in the supplementary information. 

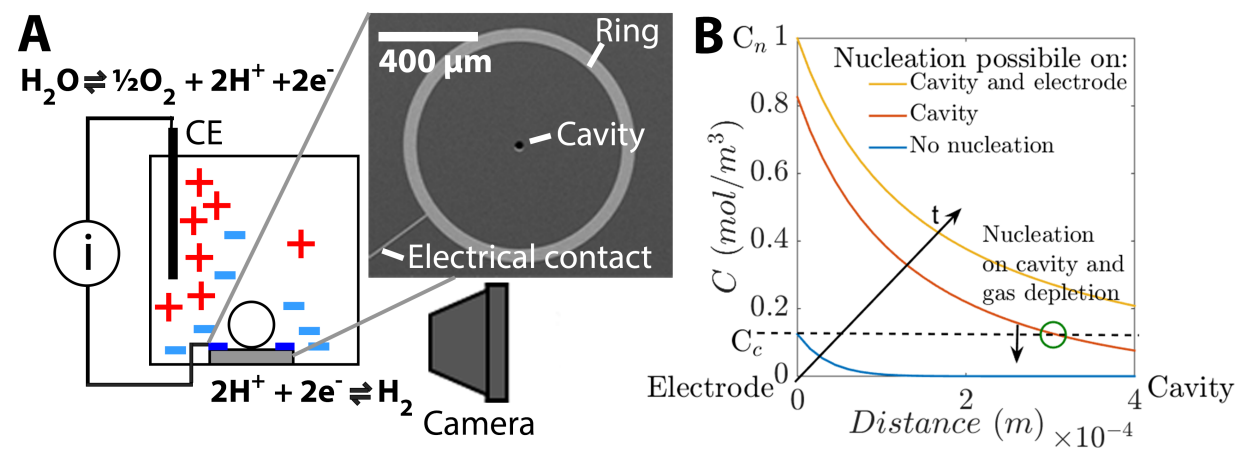

Figure 5.1: A) Experimental setup sketch (not to scale). The substrate with platinum electrode and hydrophobic cavity is situated at the bottom of a glass container $20 \times 60 \times 20 \mathrm{~mm}^{3}$, containing the counter-electrode and the electrolyte. A power source drives the electrolysis which causes bubble evolution on the substrate, recorded with a camera and microscope objective. The inset shows a scanning electron microscopy image of a substrate, showing the silicon dioxide surface with platinum electrode and superhydrophobic cavity. The platinum ring has a width of $50 \mu \mathrm{m}$, and the inner radius is $400 \mu \mathrm{m}$. The cavity has a radius of $25 \mu \mathrm{m}$ and is placed in the center of the platinum ring. A lead from an electrical contact pad (not imaged) to the platinum ring is shown in the bottom left corner. The $2 \times 2 \mathrm{~mm}^{2}$ contact pad is located on the bottom left corner of the $10 \times 10 \mathrm{~mm}^{2}$ substrate. B) Schematic representation of the gas concentration in the cavity vicinity as function of distance, the curves represent the concentration increase in time indicated by the arrow starting in the origin. The concentration required for bubble nucleation on the electrode is indicated by $\mathrm{C}_{n}$ whereas the bubble on the cavity starts growing at the dashed line $\left(\mathrm{C}_{c}\right)$ due to a lowered nucleation threshold. The growing bubble then starts to deplete the liquid of gas resulting in an overall lowered concentration indicated by the downwards arrow.

\subsection{Results and Discussion}

Hydrogen is formed on the cathode surface during electrolysis of water, dissolves in the electrolyte, and diffuses into the bulk liquid forming a concentration boundary layer. The boundary layer thickness and concentration increases with continued reaction, supersaturating the liquid with the highest concentration occurring at the electrode surface.

With increased supersaturation, bubble formation occurs on the superhydrophobic cavity before nucleation can occur on the electrode surface due to the lower thermodynamic barrier. The cavity has a lower 
thermodynamic barrier because less interfacial energy is required for the change in the radius of curvature of the gas-liquid interface at the cavity to reach critical size. This is schematically shown in Figure 5.1B where the gas concentration required for nucleation on the electrode $\left(C_{n}\right)$ is shown, as well as the concentration requirement for bubble growth on the cavity $\left(C_{c}\right.$, indicated by the dashed line) as function of the distance. The concentration evolution in time is shown by the multiple curves. After nucleation, the bubble grows by taking up gas from the dissolved gas boundary layer, depleting an area around the bubble. The gas depletion in radial direction around the bubble is proportional to $\delta=\sim\left(D_{i} t\right)^{1 / 2}$, with $D$ the diffusion coefficient for species $i$, and $t$ the time $[113,103]$. The gas depletion lowers the supersaturation level at the electrode surface (indicated in Figure 5.1B by the downwards arrow), limiting the possibility for onset of bubble nucleation on the electrode. With the electrode facing upwards the bubble growth continued until the buoyancy force overcame the interfacial tension force, after which the bubble detaches from the substrate [114]. Next, a new bubble formed on the superhydrophobic cavity. Figure 5.2A shows images of evolving bubbles, and a schematic representation of the concentration profiles in Figure 5.2B.

Figure 5.2C shows a measurement where bubble evolution took place on both, the electrode, as well as the cavity, resulting in a largely fluctuating electrical potential. In contrast, a typical electrical response measurement is shown in Figure 5.2D during electrolysis with bubble evolution on the cavity. The arrows indicate the detachment of the successive bubbles formed on the cavity. In the next section, the potential changes due to evolving bubbles are discussed in detail. 

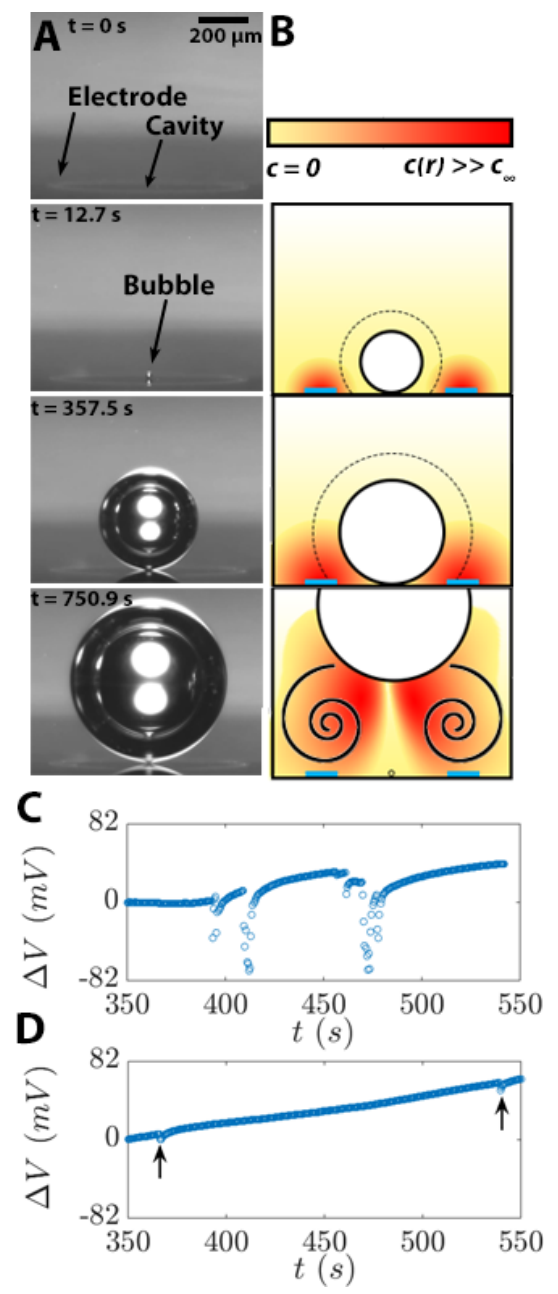

Figure 5.2: A) Images sequence of a bubble growing on the artificial nucleation site at various time intervals. Inside the bubble, dark circular shape, bright spots result from reflections of the light source. The reflection of the bubble is seen on the $\mathrm{SiO}_{2}$ substrate below the bubble neck. B) Schematic representations of the gas concentration profile in the liquid at intervals comparable to A, where the color bar indicates a low (yellow) and high (red) concentration of dissolved gas in the electrolyte around the electrode (blue). The bubble takes up gas from the surrounding liquid indicated by the dashed line. After bubble detachment, the spirals indicate the convective flow induced by the displacement of the liquid phase. C) The potential difference measured during bubble evolution on both cavity as well as the platinum ring electrode. The fluctuation of the potential is attributed to the evolution of the bubbles. A current density of $112 \mathrm{~A} / \mathrm{m}^{2}$ was applied, a $\mathrm{pH} 1$ solution was used as electrolyte, and the radius of the cavity was $5 \mu \mathrm{m}$. D) The potential difference measured during bubble evolution on the cavity alone, the electric response to bubble detachment is indicated by the arrows. A current density of $374 \mathrm{~A} / \mathrm{m}^{2}$ was applied, a $\mathrm{pH}$ 7 solution was used as electrolyte, and the radius of the cavity was $10 \mu \mathrm{m}$. 


\subsubsection{Electrical potential changes due to evolving bubbles}

Bubbles affect both the Ohmic overpotential and concentration overpotential. Therefore, the interelectrode spacing was large $(\sim 1-2$ $\mathrm{cm}$ ), to avoid fluctuations in the Ohmic overpotential introduced by micro-sized bubbles [71, 76]. Additionally, a counter electrode (CE) with an area $\left(\sim 8 \times 10^{-6} \mathrm{~m}^{2}\right)$ larger than the ring electrode (largest: $\sim 1.33 \times 10^{-7}$ $\mathrm{m}^{2}$ ) on the substrate was used. This results in a low current density in the $\mathrm{CE}$, reducing the influences of bubble evolution at the $\mathrm{CE}$ on the total overpotential. Thus, changes in the total overpotential are likely to arise only from fluctuations in the concentration overpotential caused by concentration gradients at the electrode surface.

The cathodic potential can be calculated as:

$$
E_{c}=E_{H_{2}}^{0}+\frac{2 R T}{F} \ln \left(\frac{\left[H^{+}\right]}{\left[H_{2}\right]}\right)
$$

with $E_{H_{2}}^{0}$ the standard hydrogen electrode potential, $R \approx 8.314 \mathrm{~J} / \mathrm{K} \cdot \mathrm{mol}$ the gas constant, and $T$ the temperature in $\mathrm{K}$. The cathodic potential thus changes with proton availability at the electrode surface. Figure 5.3A shows three separate measurements with different current densities forming hydrogen bubbles on the same substrate. The bubble radii and electric potential, are shown as a function of electric charge during hydrolysis ( $Q=i \times t$, with $Q$ the charge in C and $i$ the current in A). The applied current densities were; $197 \mathrm{~A} / \mathrm{m}^{2}, 393 \mathrm{~A} / \mathrm{m}^{2}$, and $786 \mathrm{~A} / \mathrm{m}^{2}$, these values were used since lower current densities resulted in slow bubble evolution, higher current densities resulted in bubble nucleation on the electrode surface by the increased gas production, reaching nucleation conditions. 

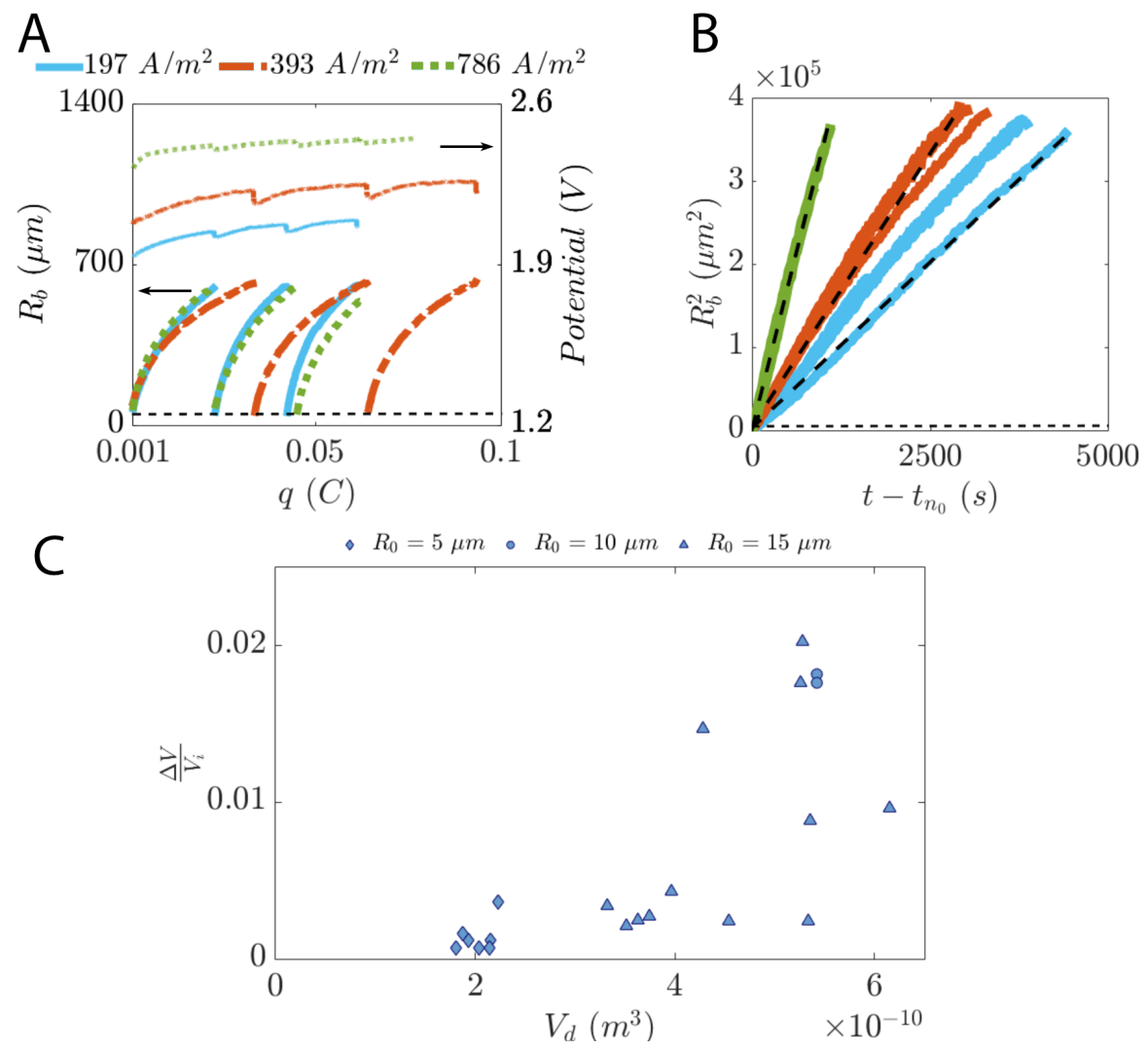

Figure 5.3: A) Bubble radii evolution (left y-axis) for current densities $197 \mathrm{~A} / \mathrm{m}^{2}, 393 \mathrm{~A} / \mathrm{m}^{2}$, and $786 \mathrm{~A} / \mathrm{m}^{2}$, with corresponding potential measurements (right y-axis). These bubbles evolved from the same substrate with a cavity radius of $\mathrm{R}_{0}=15 \mu \mathrm{m}$ in a $\mathrm{pH} 7$ electrolyte, which was renewed between experiments. The potential drops were found synchronized with the bubble detachment. The dashed line indicates the optical resolution which with the bubble radii could be determined accurately. B) The squared bubble radius (as shown in A) as function of the lifetime of a single bubble. The angled dashed black lines indicate the theoretical diffusive growth. The horizontal dashed line indicates the optical resolution limit. C) The potential drop (as obtained in A) normalized by the initial potential as function of the volume of the detached bubble. The bubbles where evolved under various current densities and from various cavity radii indicated by the different symbols (where the diamond indicates the bubbles formed from a cavity with a $5 \mu \mathrm{m}$ radius, the circle indicates the bubbles formed from a cavity with a $10 \mu \mathrm{m}$ radius, and the triangle indicates the bubbles formed from a cavity with a $15 \mu$ m radius). The larger volume bubbles tend to show larger potential drops. The reason for the scatter in results of measurements where the cavity had a radius of $15 \mu \mathrm{m}$ has been attributed to the mixing of the non-homogeneously gas saturated electrolyte and subsequent unknown concentration overpotential after electrolyte mixing. 
The bubble growth was limited by the diffusion of gases as is shown in Figure 5.3A, and evidenced by comparing the time dependent bubble radii with the theoretical diffusive growth (indicated by $R_{b} \propto \mathrm{t}^{1 / 2}$, with $R_{b}$ the bubble radius) shown in Figure 5.3B (described by Enríquez et al. (2014) [113] for bubble growth on a substrate in a homogeneously oversaturated liquid). Figure 5.3B shows the squared bubble radius $R_{b}$ as function of time $t$, minus a time offset $t_{n_{0}}$ indicating the point in time where the $\mathrm{n}^{\text {th }}$ bubble in the succession formed. The increased slope at larger current densities is due to a higher supersaturation level with more gas production, resulting in faster bubble growth. During the growth of bubbles, fluctuations in both the gas and proton concentrations at the electrode are induced by expanding bubble interfaces which causes convection [182], the reaction of protons at the electrode, and the depletion of gases in the electrolyte. The measured potential, does not reflect changes due to the aforementioned effects during bubble growth. However, potential drops (here $18 \mathrm{mV}$ to $65 \mathrm{mV}$ ) coincide in time with bubble detachment, known to induce liquid convection [182]. The liquid phase is displaced by rising bubbles and in turn, the electric double layer and dissolved gas boundary layer are disrupted. We have hypothesized that the transport of protons to the electrode could be altered by the induced convective flows. This can in turn, result in a reduced concentration overpotential and electric potential drop.

Bubble detachment from the superhydrophobic cavities occurs once the buoyancy force overcomes the interfacial tension force. Differences in detachment radii can be observed in Figure 5.2A. The varying detachment radii originated from small contact line movement $(<40 \mu \mathrm{m})$, partially discussed elsewhere $[28,27]$ and in the supplementary information. If the contact line moved beyond the apex of the cavity, the interfacial tension force increased resulting in a larger detaching bubble. Upon measurement of both the radius of the bubble area in contact with the flat silicondioxide surface and the contact angle formed, the measured bubble detachment radius was found in good agreement with the Fritz radius, as shown in Figure 5.6A.

Obviously, for a given rising bubble velocity, larger detaching bubbles induce more convection than smaller bubbles. With more convection, the disruption effect is expected to be more pronounced. To illustrate this, the volume of detaching bubbles is set out against the potential drop in Figure 5.3C. It can be seen that smaller bubbles cause small potential fluctuations and with increased bubble volume the potential drops increase, as well as the spread in the measured potential drop. The spread in measured potential drops could be attributed to the mixing of a non-homogeneously hydrogen saturated electrolyte. As the boundary layer is restructured by 
convection-induced mixing with the bulk electrolyte, small fluctuations on the size and trajectory of the detaching bubbles would lead to differences in dissolved gas concentration near the electrode, hence resulting in fluctuations in the concentration gradient.

\subsection{Summary}

The location of bubble formation was spatially controlled near a novel ring electrode design. As a result, we demonstrated gas evolution with bubble formation away from the electrode surface. The bubbles grew via diffusion until detachment occurred, upon which convection was induced by the rising bubble. The convection disrupted the gas boundary layer near the electrode, and a potential drop proportional to the detached bubble size was measured due to a lowered concentration overpotential. Fluctuations in potentials other than those associated to the bubble detachment that are typically present when bubbles directly form on the electrode surface are here avoided. Keeping the reactive surface free of bubble formation and minimizing potential fluctuations can ultimately lead to a higher Faradaic efficiency in water electrolysis and other gas-evolving electrochemical reactions.

\section{Acknowledgments}

The authors would like to thank Wouter Vijselaar and Stefan Schlautmann for the substrate fabrication. We would like to further extend our thanks to Remco Sanders, Loreto Oyarte Galvez, Katharina $\mathrm{Cu}$, and Haye Witteveen for their help with the equipment used.

This work was supported by the Netherlands Center for Multiscale Catalytic Energy Conversion (MCEC), an NWO Gravitation programme funded by the Ministry of Education, Culture and Science of the government of the Netherlands. 


\section{Appendix A: Supplementary information}

\section{Micromachined $\mathrm{SiO}_{2}$ substrates with $\mathrm{Pt}$ electrodes and superhydrophobic cavities}

Figure 5.4A shows cross-sectional sketches of the steps taken for substrate fabrication. In step 1 , a silicon wafer (gray) with $150 \mathrm{~nm}$ thick $\mathrm{SiO}_{2}$ layer (blue) was patterned using standard photolithography techniques. The Olin OIR 907-17 resist (red) was spin-coated, followed by DC-sputtering of a 10 $\mathrm{nm}$ thick tantalum adhesion layer and $100 \mathrm{~nm}$ thick platinum layer (purple) with a custom made sputter coater (T'COaty, Nanolab, MESA+ Institute). Step 2 shows the substrate after the deposited metal has been patterned via a lift-off process, the resulting metal film forms ring shaped electrodes with an inner radius of $400 \mu \mathrm{m}$ and widths that varied per electrode between 10 $\mu \mathrm{m}$ and $50 \mu \mathrm{m}$. Superhydrophobic cavities with radii between $5 \mu \mathrm{m}-25 \mu \mathrm{m}$ were created (steps 3 and 4 ) in the center of each electrode. The fabrication of superhydrophobic cavities has been described elsewhere [28, 27]. Finally, the wafer is cleaned in acetone with an ultrasonic bath (VWR Ultrasonic Cleaner USC-THD, $45 \mathrm{kHz}$ ) and diced (Dicing Saw Loadpoint Micro Ace 3) to form $10 \times 10 \mathrm{~mm}^{2}$ substrates (step 5).

\section{Sample preparation and electrochemical setup}

All substrates with platinum electrode and superhydrophobic cavity were cleaned for 10 minutes, using an ultrasonic bath (Elmasonic S30H, 50/60 $\mathrm{Hz}$ ) with acetone, after which the samples were rinsed with deionized water followed by acetone. The rinsing was repeated three times followed by a final deionized water rinse before the substrate was dried with $\mathrm{N}_{2}$ gas. Each substrate was glued at the bottom of a glass container with an electrical wire soldered to the electrical contact pad on the substrate bottom surface. In each experiment, $15 \mathrm{~mL}$ of electrolyte was placed inside the container, reaching a $6 \mathrm{~mm}$ layer of electrolyte above the substrate. A platinum wire counter electrode (CE), with a surface area much larger than the platinum ring, was placed into the electrolyte with an interelectrode spacing of several $(1-2 \mathrm{~cm})$ centimeters. The substrate and counter electrode were connected to a Keithley 2410 power source. Bubbles formed during hydrolysis were imaged using backlighting (Schott KL 2500 LCD), a camera (FLIR FL3-U3-32S2M-CS), and a high speed camera (Photron Fastcam SA-X2). 

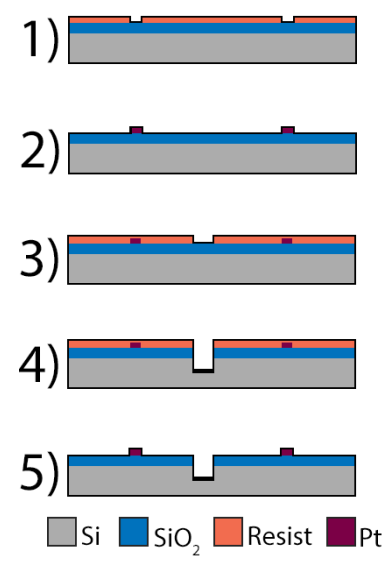

Figure 5.4: Cross-sectional overview of fabrication steps, not to scale. Step 1 shows an oxidized silicon wafer its surface covered by a patterned photoresist layer. Step 2 shows the substrate after the platinum electrode deposition. In another lithography step, shown in step 3, the areas for the cavities were defined. The superhydophobic cavity was formed, shown in step 4. Step 5 shows the finalized substrate after the cleaning step.

Two distinct situations were recorded during the bubble growth experiments. One, where the contact line of the bubble is pinned at the cavity edge, shown in Figure 5.5A, with contact angle of $90^{\circ}$. In the other, the bubble initially formed on the cavity but the contact line moved onto the $\mathrm{SiO}_{2}$ surface, forming a $25.3^{\circ}$ contact angle between gaseous and solid phase, Figure 5.5B. The reason for this could be that the contact line did not pin to the surface but rather slided over it. As expected from the equation in Figure 5.6, the larger footprint in the second scenario results in larger interfacial tension forces, and in turn larger detachment radii of bubbles. 


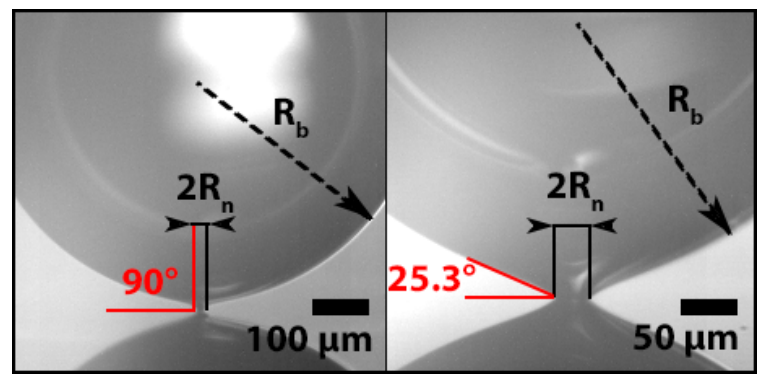

Figure 5.5: Two situations observed in which bubbles are pinned to the substrate. A) The contact line of a bubble pinned to a $15 \mu \mathrm{m}$ radius cavity with a contact angle of $90^{\circ}$. The reflection of the bubble is shown at the bottom by the black half circle starting under the neck of the bubble. B) A bubble formed on the cavity after which the contact line has moved beyond the cavity radius. The result is a contact angle of $25.3^{\circ}$.

Figure 5.6A shows the detachment radii of bubbles with corresponding neck radii $\left(\mathrm{R}_{n}\right)$ from the same electrode substrate. The bubbles evolved in various electrolytes indicated by the colours of the markers: blue for $\mathrm{pH} 1$, green $\mathrm{pH} 3$, and red for $\mathrm{pH} 10$. The current densities are indicated by circles $\left(112 \mathrm{~A} / \mathrm{m}^{2}\right)$ and triangles $\left(187 \mathrm{~A} / \mathrm{m}^{2}\right)$. The numbers next to the markers indicate the number of bubble detached in the specific succession. It should be noted that with increased number of detached bubbles, on average a smaller neck radius was measured, independent from the current density or $\mathrm{pH}$. It is still unclear to us why the bubble pinning point (measured the bubble bubble neck) initially expand beyond the cavity, as well as it is unclear why as more bubbles evolve from the cavity we observe the bubble neck expanding less. The reasons behind the scatter in detachment radii has been partially discussed elsewhere [28, 27]. The dashed lines in Figure 5.6A, depict the detachment radii expected for a contact angle of 26.5 (top dashed line) and 13 degrees (bottom dashed line). These contact angles were arbitrarily chosen to illustrate the theoretical detachment calculated by the detachment equation [114] in the graph. 

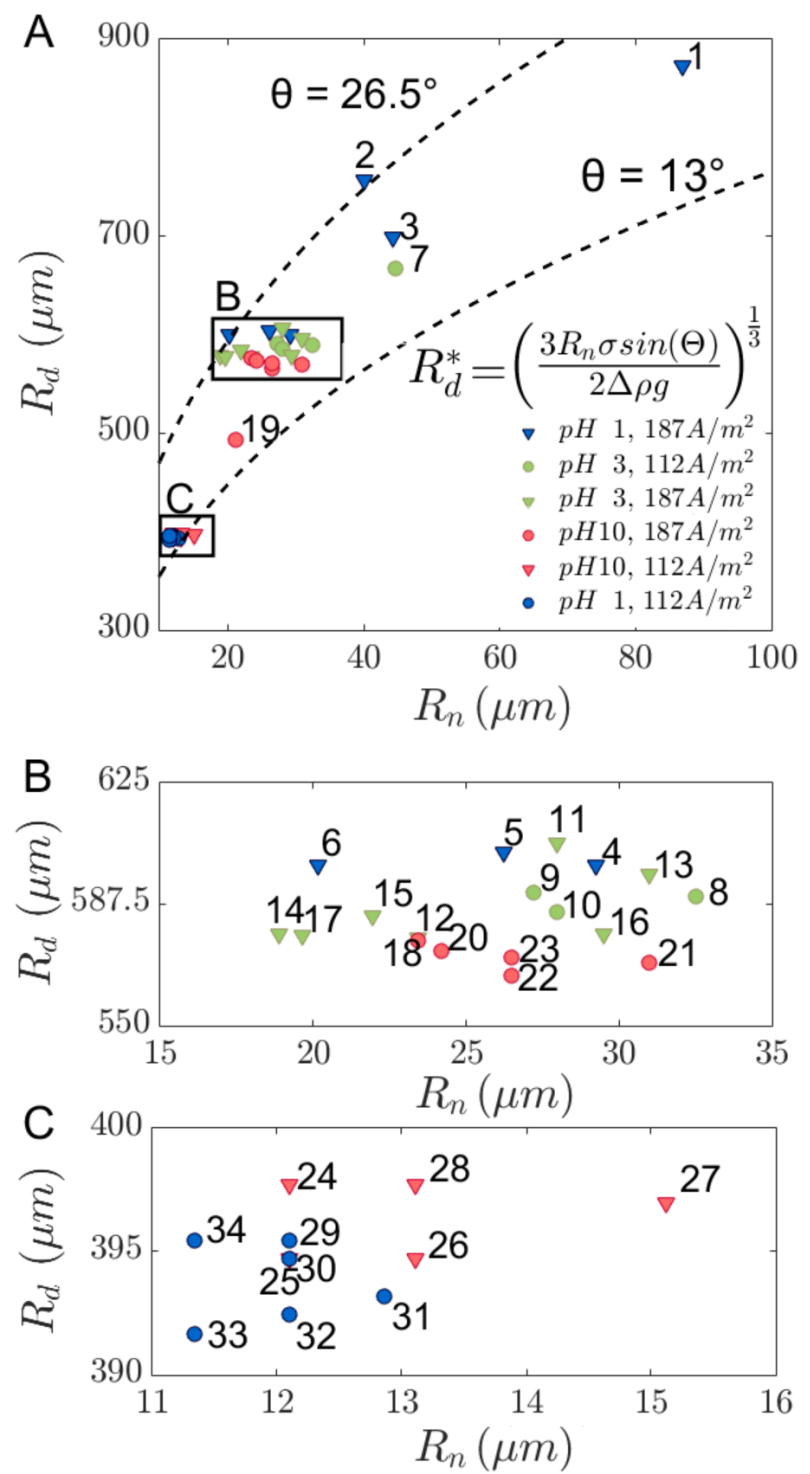

Figure 5.6: A) Detachment radii of six measurements on a single sample. The legend indicates from top to bottom the order in which order the measurements were done. The markers are labeled with a number, indicating the order in the succession of bubble detachment. The equation shows the theoretical bubble detachment radius from balancing the interfacial force and buoyancy force. The clusters of markers in the rectangles are enlarged in the subfigures, and an arrow indicating the decrease in bubble radii with each new detachment. 


\section{Perspectives for future studies and conclusions}

This chapter discusses perspectives for future studies of electrolyzers employing artificial nucleation sites, and general conclusions on the work covered in this thesis. 


\subsection{Introduction}

Scaling up the amount of nucleation sites and electrode surface is a logical step to start bridging the gap between fundamental research to more practical electrolyzer systems. We show the initial findings on the nucleation, growth, and detachment of bubbles in such systems. Closed system electrolyzers allow for preciser control of conditions such as pressure in the electrolyzer. Also liquid/air interfaces are smaller in size and therefore the outflux of dissolved gases from the electrolyzer via diffusion is minimized. Here we show a closed cell membraneless electrolyzer where applied flow separates the products formed. We aim to study the product crossover with the use of fluorescence microscopy. Additionally, we implement artificial nucleation sites for bubbles to evolve from within a glass substrate.

We separated this section in two parts, each part presenting results on a different topic with individual preliminary conclusions. The first section covers the investigation on electrolyzers employing multiple artificial nucleation sites nearby gas evolving electrodes. The second section presents the results of the closed membraneless electrolyzer fitted with artificial nucleation sites within the fluidic channel.

\subsection{Electrolyzers with multiple nucleation sites}

We have previously shown (Chapter 5) that with the use of superhydrophobic cavities located nearby electrodes with a surface area of $1.34 \times 10^{-7} \mathrm{~m}^{2}$, those surfaces remained free from bubble nucleation throughout the electrolysis of water. Here we use larger planar electrodes (2 electrodes of $5000 \times 10^{-6} \mathrm{~m} \times 70 \times 10^{-6} \mathrm{~m}$ ) to investigate the possibility to prevent bubble nucleation on the gas evolving electrodes using multiple superhydrophobic cavities. In addition we investigate the overpotential changes during bubble evolution.

Bubbles evolving next to electrodes have been granted little attention in literature, however bubbles on electrodes have been investigated (and reviewed in Chapter 2). Literature reporting bubbles residing on electrodes showed that the resistance overpotential is increased, which was attributed to the screening of the electrode surface by the presence of a bubble [197, 198, 71, 73]. Also, the increased resistance has been attributed to the current path being obstructed close to a bubble surface [74].

Dukovic et al. (1987) [69] proposed a model that took into account not only the resistive overpotential effects, but also the concentration overpotentials. The concentration overpotential arises with the formation of a dissolved gas boundary layer in which a bubble forms [70]. Bubbles grow 
by taking in gas from their surrounding liquid, upon which the boundary layer is locally depleted [28]. The concentration overpotential can thus vary with the dissolved gas concentration which can be lowered by bubbles. The model presented by Dukovic et al. predicted this occurrence by describing a negative differential overpotential arising from the increased resistance overpotential and lowered concentration overpotential under fast electrode kinetics (a dimensionless exchange current density of $6.8 \times 10^{-4}$ ), and low current densities. Gabrielli et al. (2005) [70] experimentally confirmed later the existence of the negative differential overpotential.

Upon bubble detachment, changes in overpotential can arise due to the boundary layer near the electrode being disrupted by convection induced by the detaching bubble (previously described in Chapter 5 ). The convection can mix the high concentration gas boundary layer that resides close to the electrode surface during stationary electrolyte conditions, with the lower gas concentration liquid from the bulk. As a result, gas and proton concentration at the electrode surface decrease.

\section{Micromachined $\mathrm{SiO}_{2}$ substrates with $\mathrm{Pt}$ electrodes and multiple superhydrophobic cavities}

Figure 6.1A illustrates the various fabrication steps. Shown in step 1, thermal oxidized silicon ( $150 \mathrm{~nm}$ thick $\mathrm{SiO}_{2}$ ) wafers $<100>$ were patterned using standard photolithography techniques. Using dc-sputtering (T'COaty, MESA+ nanolab, custom made sputter coater), subsequently a $10 \mathrm{~nm}$ thick tantalum layer and $100 \mathrm{~nm}$ thick platinum layer were deposited (yellow). It is shown in step 2 that removing the resist and the metal not in contact with the $\mathrm{SiO}_{2}$ layer, using a lift-off technique, resulted in two electrodes $(70 \mu \mathrm{m} \times 5000 \mu \mathrm{m})$ per sample. Superhydrophobic cavities with radii of $25 \mu \mathrm{m}$ were created next to the electrodes. This process step has been reported elsewhere [27] but is also illustrated in step 3 where a standard photolithography step is shown. The amount of cavities has been varied, between 2 and 9 cavities near each electrode, over the various designs. The wafer was cleaned and diced to $10 \mathrm{~mm} \times 10 \mathrm{~mm}$ samples, shown in step 4 .

Figure 6.1B shows a schematic top view of a finalized sample, the varied parameters are indicated by $S_{e}, \mathrm{~S}_{e p}$, and $S_{p}$ for the electrode spacing, electrode-cavity spacing, and intercavity spacing, respectively. 

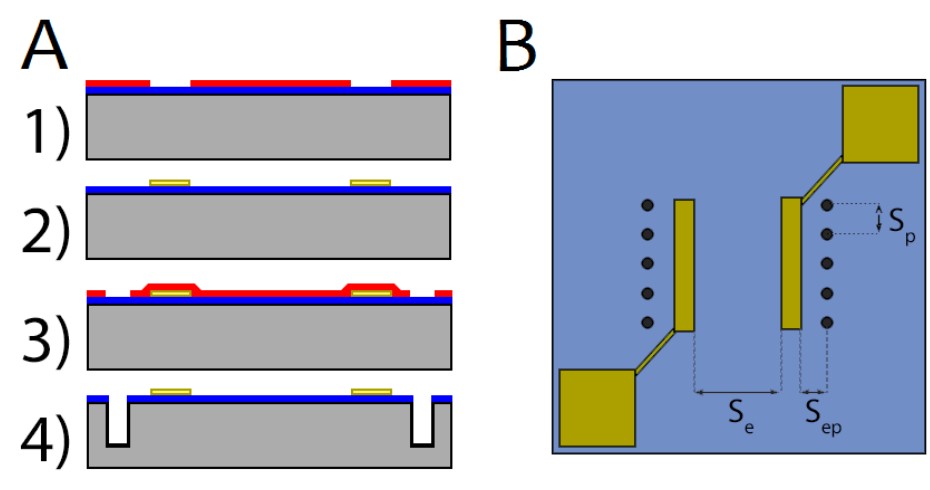

Figure 6.1: A) Cross sectional view (not to scale) of the fabrication steps for multifunctional electrodes with super hydrophobic cavities nearby the electrodes. Step 1 show the silicon wafer (gray) with a thermal oxide layer (blue) of $150 \mathrm{~nm}$ on top with a resist layer (red) that has been patterned with standard photolithography steps. Step 2 shows the substrate after depositing $10 \mathrm{~nm}$ tantalum and subsequently $100 \mathrm{~nm}$ platinum followed by a lift-off technique removing the metal that was deposited upon the resist. Step 3 shows a second photolithography step in which the areas where the superhydrophobic cavities should be formed are defined. Step 4 shows the substrate after the fabrication of the superhydrophobic cavities and subsequent cleaning of the sample. B) Schematic top view (not to scale) of the finalized sample. The electrode (light blue) spacing $S_{e}$ has been varied over the designs, as well as the electrode-cavity spacing $\left(S_{e p}\right)$ and the spacing between cavities $S_{p}$.

\section{Optical imaging setup}

Figure 6.2 shows a cross sectional overview of the setup used to measure bubble formation nearby planar electrodes. A polylactic acid 3D printed holder (light gray) is shown, the two parts where combined with the use of screws (not depicted). The sample (dark blue) was placed between the two parts of the holder with a polytetrafluoroethylene (PTFE) layer (yellow) in between to create a liquid tight seal between the sample and holder to contain the $1 \mathrm{M} \mathrm{H}_{2} \mathrm{SO}_{4}$ (light blue). Next, the sample is connected via electric wires soldered to contact pads on the substrate and the wires are connected to a Keithley 2410 power source. A camera (dark gray) is used to record a top view of the sample and evolving bubbles. 


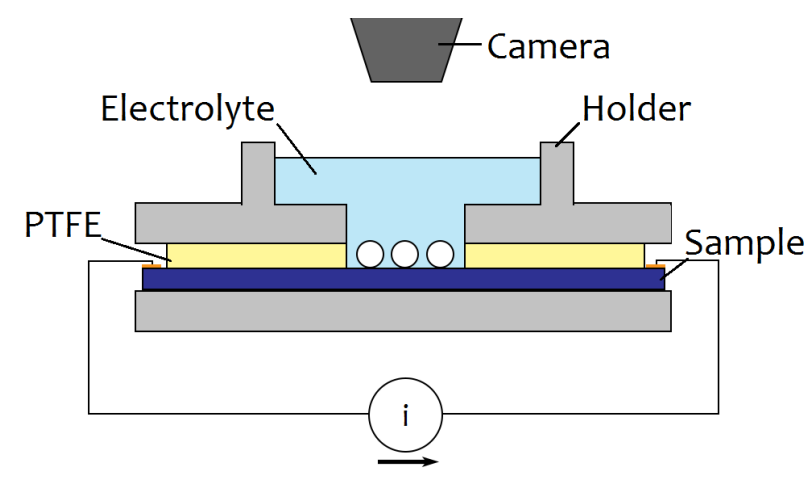

Figure 6.2: Cross sectional view of the experimental setup (not to scale). The polylactic acid 3D printed holder (light gray) contained the $1 \mathrm{M} \mathrm{H}_{2} \mathrm{SO}_{4}$ electrolyte (light blue) and sample (dark blue) with a PTFE liquid tight seal. A camera (dark gray) is used to record the bubble formation during water electrolysis powered with a Keithley 2410 power source.

\section{Bubble evolution}

We investigated the bubble evolution from superhydrophobic cavities next to the electrodes. In Figure 6.3 the bubbles formed on superhydrophobic cavities with radii of $25 \mu \mathrm{m}, 250 \mu \mathrm{m}$ near the electrodes, are shown after 384 seconds of water electrolysis. The artificial nucleation sites have been numbered, with the site numbers indicated next to the evolving bubbles.

Bubble nucleation occurred only on the cavities, keeping the $1 \mathrm{~mm}$ separated anode and cathode free of bubble evolution. The bubbles formed near the cathode are larger in size than those formed near the anode, this was attributed to the stochiometry of the reaction (Equation 1.1 and Equation 1.2) and the larger diffusivity of the hydrogen gas in water. Furthermore, the bubble size increases from left to right at both electrodes. However, the Ohmic resistance along the electrode is expected to increase from the start to end of the electrodes (from left to right for the cathode and right to left for the anode). Therefore, the gas production rates are expected to be highest at the start of the electrodes as well as the growth rates of the bubbles. The observed gas bubble sizes do not adhere to this expectation which might be the result of gas product cross-over from one electrode to another or the presence of specific domains with larger than average gas production rates on the electrodes. 


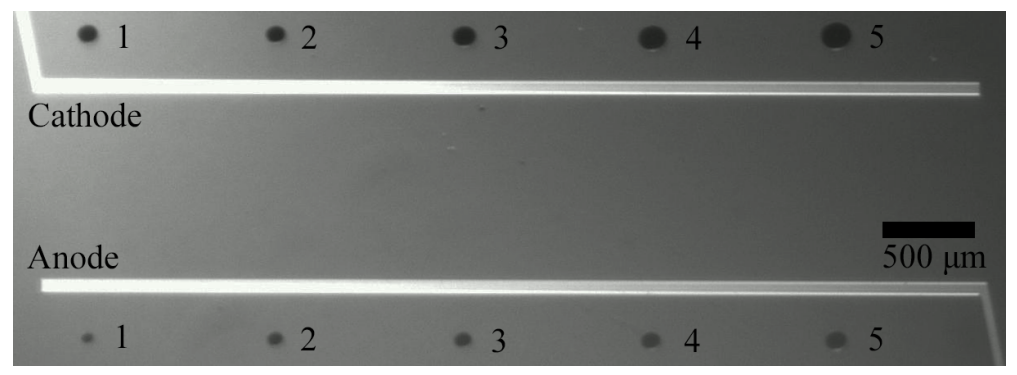

Figure 6.3: Top view of bubbles formed on superhydrophobic cavities after $384 \mathrm{~s}$ of water (deionized water and $1 \mathrm{M} \mathrm{H}_{2} \mathrm{SO}_{4}$ ) electrolysis at a constant potential of $1.6 \mathrm{~V}$. At the top, the cathode and corresponding hydrogen bubbles on the artificial nucleation sites labeled 1 to 5 . At the bottom, the anode and corresponding oxygen bubbles on the artificial nucleation sites labeled 1 to 5 . The spacing between the centers of the sites is $1000 \mu \mathrm{m}$, the spacing between the sites and closest electrode surface is $250 \mu \mathrm{m}$, and the interelectrode spacing is $1000 \mu \mathrm{m}$.

\section{Bubble nucleation}

Figure 6.4 shows the average nucleation time $\overline{t_{n}}$ as function of the current density $J$ for both hydrogen and oxygen bubbles originating from various experiments with various samples. The nucleation times of hydrogen bubbles and oxygen bubbles showed large deviations over all galvanostatic measurements in a $1 \mathrm{M} \mathrm{H}_{2} \mathrm{SO}_{4}$ electrolyte. The extrema in the measured times are indicated per current density by the error bars. The deviation was attributed to the Ohmic drops along the electrodes, sample to sample differences, and the concentration of dissolved gas in the boundary layer, which could be affected if bubbles were already present when bubbles nucleate on another site first. With increased current density the average nucleation times for both gases was found to decrease as expected. The average nucleation times for hydrogen bubbles were found to be lower than those of oxygen bubbles. It stands to reason that the stochiometry, the diffusivity of the two gases in water (diffusion coefficient of $4.5 \times 10^{-9} \mathrm{~m}^{2} / \mathrm{s}$ and $1.97 \times 10^{-9} \mathrm{~m}^{2} / \mathrm{s}$ for hydrogen and oxygen gas, respectively [50]), and the gas solubility (the mole fraction solubility of hydrogen and oxygen gases in water at STP is $1.411 \times 10^{-5}$ and $2.3011 \times 10^{-5}$, respectively [199]) play a large role in the supersaturation level at the superhydrophobic cavities. Therefore, higher supersaturation levels at the cathode than at the anode are expected, and earlier onset of bubble nucleation near the cathode. 


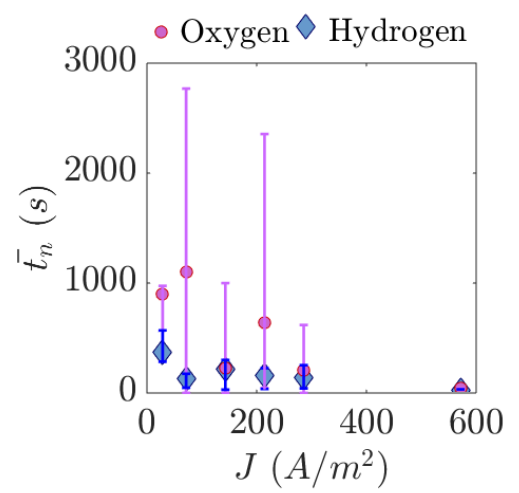

Figure 6.4: The average nucleation time as function of the current density is shown for both oxygen (red circles) and hydrogen (blue diamonds) bubbles formed in galvanostatic measurements in an $1 \mathrm{M} \mathrm{H}_{2} \mathrm{SO}_{4}$ electrolyte.

\section{Electrolytic bubble growth}

Figure 6.5A shows the first evolved hydrogen bubble radii $R_{b}$ as function of time for the experiment depicted in Figure 6.3. The different colors indicate which bubble evolved from which site near the electrode. Similarly, Figure 6.5B shows the oxygen bubble radii as function of time. Figure $6.5 \mathrm{C}$ shows the logarithmic values plotted, corresponding to A, of the hydrogen bubble radii over the cavity radius as function of the time, and in Figure 6.5D the logarithmic values are plotted for Figure 6.5B. The logarithmic values are presented since typical diffusive growth profiles of bubbles growing in gas saturated liquid follow $R_{b} \propto \sqrt{t}$ and form a straight line with a slope of 0.5 , indicated by the black lines in Figures 6.5C and D.

Both hydrogen and oxygen bubbles did not evolve following the typical diffusive growth profile, instead we found that the growthrate varied with time. Several reasons for this could be considered; first, regions on the electrodes with locally higher reaction rates could favor the formation of a non-homogeneously evolving boundary layer which in turn can be depleted by the growth of 10 bubbles simultaneously. Also the interaction of bubbles via Ostwald ripening (a process in which a larger bubble takes in gas from a smaller one) can be considered since the regions around the bubbles where gas is taken from in radial direction is $\delta \propto \sqrt{D_{i} t}$, with $D_{i}$ the diffusion coefficient of species $i$, and $t$ the time [28], this will overlap with neighboring bubbles' regions after $\sim 17.7$ seconds in the case of hydrogen bubbles. Furthermore, experiments forming multiple successive bubbles were affected by the desynchronization between bubbles. A bubble forming after the first bubble detached formed in the presence of bubbles 


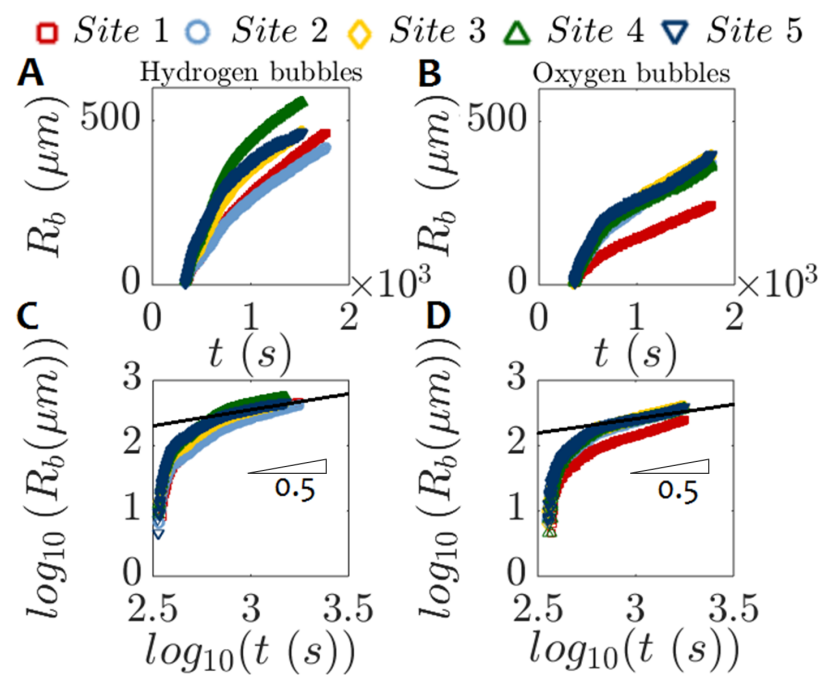

Figure 6.5: The first bubble evolution per site near both cathode and anode. A) The radius of the first hydrogen bubble evolution from each cathode site as function of time. Nucleation of the bubbles started between $330 \mathrm{~s}$ and $355 \mathrm{~s}$. Detachment occurred with radii between $417 \mu \mathrm{m}$ and $556 \mu \mathrm{m}$ via coalescence. B) The radius of the first oxygen bubble evolution from each anode site as function of time. Nucleation occurred with $350 \mathrm{~s}$ to $375 \mathrm{~s}$, on average later for oxygen bubbles than for hydrogen bubbles. No detachment occurred near the anode during the measurement. C) The logarithmic values of (A) are plotted with the black line indicating the growth profile following $R_{b} \propto \sqrt{t}$. D) The logarithmic values of (B) are plotted with the black line indicating the growth profile following $R_{b} \propto \sqrt{t}$.

that had not detached yet. Resulting in the growth being affected by the aforementioned effects caused by other bubbles, the growth profiles are found to be similar to those shown in Figure 6.5.

It should be further noted from comparing Figures $6.3 \mathrm{~A}$ and $\mathrm{B}$ that the stochiometry is tilted in favor of hydrogen bubbles but not in a 2:1 ratio. This is the possible result of not all the gas going to the bubbles but rather to saturating the liquid, the reverse reaction, and possible formation of bubbles on the PTFE layer. 


\section{Bubble detachment}

The spacing between cavities of $S_{p}=1000 \mu \mathrm{m}$ is smaller than the combined Fritz radii (Equation 3.9) of two neighboring sites. With the Fritz radius $R_{F}$ $=644 \mu \mathrm{m}$ (under the assumption of a $90^{\circ}$ contact angle, for $R_{0}=25 \mu \mathrm{m}$ ), coalescence is expected to be the main mechanism to induce detachment in the first bubbles formed. After the first bubbles evolved, variations in detachment times arise due to the divergence in nucleation times and growth rates. This desychronized bubble evolution resulted in a situation where bubbles did not coalesce, but detachment occurs when the buoyancy force exceeds the interfacial tension force.

Figure 6.6 shows current fluctuations (orange line) coinciding with bubble detachment in a $1 \mathrm{M} \mathrm{H}_{2} \mathrm{SO}_{4}$ solution during a measurement in which the potential is kept constant at $2.35 \mathrm{~V}$ (blue line). Here the electrode spacing $S_{e}=1000 \mu \mathrm{m}$, the spacing between electrode and pit $S_{e p}=250 \mu \mathrm{m}$, and $S_{p}=1000 \mu \mathrm{m}$. The current decreases with time due to electrode polarization and upon bubble detachment (indicated by the arrows) fluctuation of max. $\sim 0.3 \mu \mathrm{A}$ in the measured current is observed. Upon the last bubble detachment, indicated by the fifth arrow, a negative current drop can be seen. Upon detachment of bubbles convection can be induced [182], here the response in current fluctuation upon bubble detachment can be the result of the disruption of the dissolved concentration boundary layer at the electrode by the induced convection (as discussed in Chapter 5).

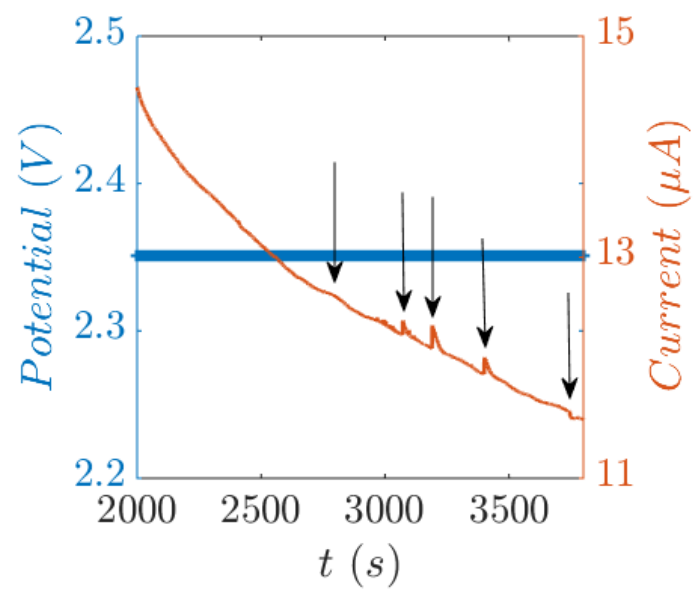

Figure 6.6: Current fluctuation upon bubble detachment during contstant $2.35 \mathrm{~V}$ potential (blue). Due to electrode polarization the current (orange) decreases with time, upon bubble detachment (indicated by the arrows) current fluctuations occurred. 
It stands to reason that upon the detachment of bubbles the induced convection scales with the volume of the detaching bubbles, since with larger bubbles more liquid is displaced upon bubble detachment. Figure 6.7 shows the normalized current drop (the current difference between before and immediately after bubble detachment $(\Delta i)$, normalized by the current before bubble detachment $\left(i_{i}\right)$ ) as function of the volume of the bubbles detaching. Here the electrode spacing is $S_{e}=1000 \mu \mathrm{m}$ and the spacing between electrode and pit $S_{e p}=500 \mu \mathrm{m}$.

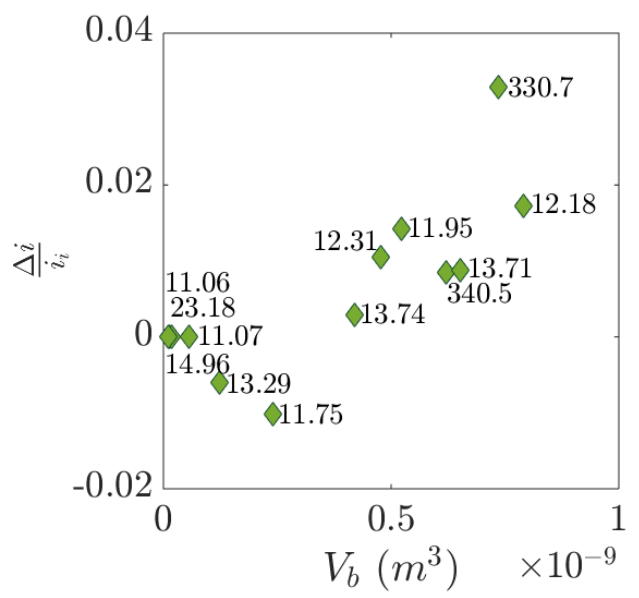

Figure 6.7: The current difference between after and before bubble detachment $(\Delta i)$, is normalized by the current before bubble detachment $\left(i_{i}\right)$. The normalized current is shown as function of the detaching bubble volume with the assumption of a spherical bubble. The number next to each data point indicates $i_{i}$, the current before bubble detachment.

The variation in $i_{i}$ does not show any correlation to the measured normalized current difference $\left(\Delta i / i_{i}\right)$. However, $\Delta i / i_{i}$ converges to zero with a decrease in bubble volume. The measured spread in the data is attributed to the mixing of the boundary layer of dissolved gas with the bulk liquid. Liquid with high and low gas concentrations are mixed together forming the new concentration at the electrode resulting in a new concentration overpotential after mixing. Therefore, the unknown contribution of the concentration and resistance overpotential before and after the bubble detachment determines the height of the current fluctuation. 


\section{Bubble movement}

Occasionally, movement of bubbles from superhydrophobic cavities occurred by the bubbles moving closer to gas evolving electrodes while growing. This was observed at both anode and cathode of the electrolysis cell. In Chapter 5, bubble contact line movement was observed toward the ring electrodes, which could be the case here as well. Figure 6.8A shows the movement of a hydrogen bubble during electrolysis with a constant potential of $2.35 \mathrm{~V}$, in a $1 \mathrm{M} \mathrm{H}_{2} \mathrm{SO}_{4}$ electrolyte. A change in surface tension by electric fields and gas concentration could result in a lowered interfacial tension force and Marangoni flow from high to low surface tension areas [124]. A temperature gradient as result of Joule heating would decrease the surface free energy locally and the density of the liquid which can result in flow [200]. The temperature increase at the electrode is considered unlikely due to the low current density of $\sim 37 \mathrm{~A} / \mathrm{m}^{2}$ applied, resulting in low Joule heating values and the ease of heat dissipation in the liquid. It should be noted that other undetermined parameters might influence the movement of bubbles.

Experiments using alcohol, to reduce the surface tension, around evolving bubbles resulted in the simultaneous introduction of local flow and additional nucleation sites on capillaries used to inject alcohol close to evolving bubbles. Therefore, these experiments were inconclusive and deemed unfit in their current form.

Figure $6.8 \mathrm{~B}$ shows the distance from the center of the bubble, determined by the top view, to the electrode edge (in blue) and the radius of the growing bubble (in orange) are set out as function of time. The bubble nucleated on the superhydrophobic cavity, $250 \mu \mathrm{m}$ distance away from the electrode surface and continues to move to a distance of $87 \mu \mathrm{m}$ removed from the electrode. This was followed by the detachment of the bubble. No changes to the surface during and after reaction were found with optical microscopy nor afterwards with scanning electron microscopy.

\section{Preliminary conclusions}

With the bubble formation occurring on the superhydrophobic cavities next to the electrodes, the electrode surfaces were kept free of bubbles with the experimental parameters applied. Hydrogen bubbles nucleated on average faster on the superhydrophobic cavities than oxygen bubbles under the same conditions, attributed to the stochiometry, solubility, and the diffusivity of the gases in water. The nucleation times for both hydrogen and oxygen bubbles decreased with increased current density due to the higher product formation rates. The bubble growth did not evolve with the square root of time, attributed to gas boundary layers not developing 

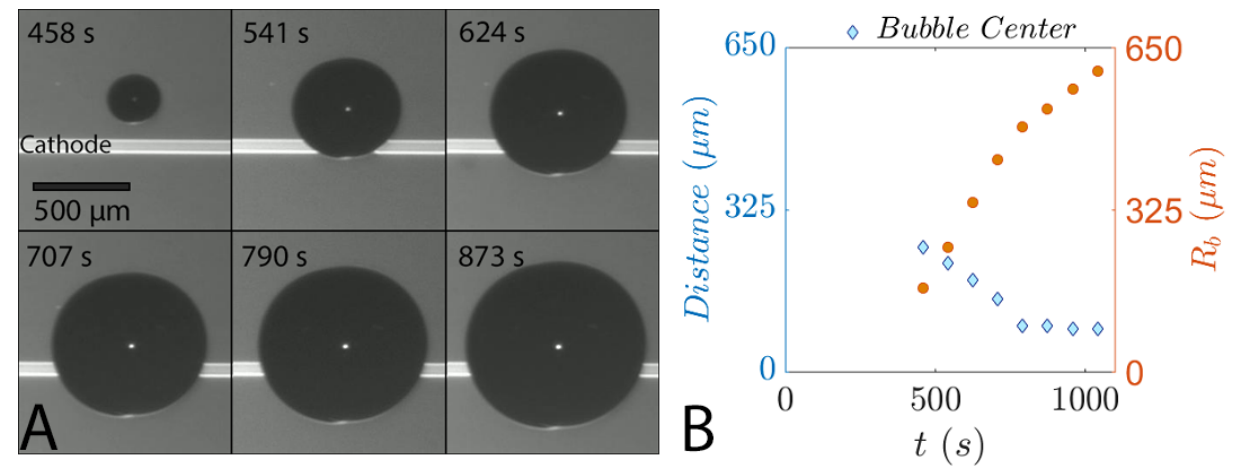

Figure 6.8: Hydrogen bubble evolution on a superhydrophobic cavity. A) From left to right and top to bottom, the growth of a hydrogen bubble over time. During the growth, the center of the bubble moves towards the cathode. B) The distance between the center of the bubble and electrode (in blue) and the radius of the bubble (in orange) are shown as function of time. At $t=0 \mathrm{~s}$ the electrolysis reaction started, nucleation on the superhydrophobic cavity $250 \mu \mathrm{m}$ away from the cathode started after $\sim 450$ s. The bubble center moved up to $87 \mu \mathrm{m}$ from the electrode till the bubble detached.

homogeneously over the electrodes, the evolving bubbles mixing the gas boundary layer, and gas crossover from one electrode to another. During the growth of bubbles, movement towards the electrode occurred sporadically. A change in surface tension by the dissolved gases was suspected to be the driving force, however the origin remained elusive since other effects cannot be excluded.

Bubble detachment, of the first bubble per site in each experiment, predominately occurred via coalescence of two bubbles due to the close spacing of the superhydrophobic cavities. Upon bubble detachment in potentiostatic measurements, positive increases in the measured current were observed which was attributed to lowered overpotentials by induced convection. Negative current drops were found to be in agreement with existing models and experiments on bubble evolution with high reaction kinetics and low current densities. Here the resistance overpotential was reported to be a positive, whereas the concentration overpotential decreased due to the bubble induced convection (partially) removing the gas boundary layer at the electrode surface. Therefore, the sign of the current drop was attributed to the concentration overpotential difference before and after bubble detachment. With smaller bubbles the current drops became smaller in magnitude, this was attributed to less convection arising from the smaller detaching bubbles. 


\subsection{Closed system electrolyzer}

Membraneless electrolyzers operating with a flow driven product separation mechanism have shown high efficient product separation [66]. However, efficiency losses by product crossover have been reported and attributed to diffusive processes. Here we have implemented superhydrophobic cavities in membraneless electrolyzers in order to study the product evolution, product crossover, and the influence of bubbles forming on artificial nucleation sites.

In membraneless water electrolyzers protons have to transfer through the liquid from the anode to the cathode to continue the water splitting reaction. Here the evolving gases are separated using flow, due to the Poiseuille velocity profile in the microfluidic channel, calculated elsewhere for a membraneless electrolyzer with same dimensions (the main fluidic channel has a width of $275 \mu \mathrm{m}$, the T-junction channel width is $100 \mu \mathrm{m}$, and the height of the channel is $61 \mu \mathrm{m}$.) [66]. The flow should therefore be large enough to keep the products separated, but protons are required at the cathode to be able to carry out the half reaction.

\section{Flow cell fabrication}

A SCHOTT MEMpax $100 \mathrm{~mm}$ diameter wafer was cleaned with a $\mathrm{HNO}_{3}$. Using Olin OiR 907-35 resist and standard photolithography steps, the electrode pattern was defined on the glass substrate. Metal films of 10 $\mathrm{nm}$ thick tantalum and $100 \mathrm{~nm}$ thick platinum were DC-sputtered on the substrate. Via a lift-off technique the resist and excess metal was removed resulting in separated electrodes.

A silicon $<100>$ substrate, was cleaned in a $\mathrm{HNO}_{3}$ bath and a subsequent 1 minute HF bath. Using standard photolithography a $1.7 \mu \mathrm{m}$ thick photoresist layer was deposited which was patterned using UV light and a mask. The UV exposed photoresist was removed and using a deep reactive ion etch (DRIE) step, $60 \mu \mathrm{m}$ deep structures trenches were created in the silicon substrate, which will function as microfluidic channels with a T-juction. The channel to the outlets is $100 \mu \mathrm{m}$ in width and has a length of $6 \mathrm{~mm}$, the main fluidic channel has a width of $275 \mu \mathrm{m}$ and a length of $10 \mathrm{~mm}$. The back side was patterned using standard photolithography, exposing circular areas with $2.5 \mathrm{~mm}$ radius. In a subsequent DRIE step, the circles were etched down to form through substrate holes, which will function as in- and outlets for the connections.

The design made use of superhydrophobic cavities with a radius of 2.5 $\mu \mathrm{m}$. They were formed, using DRIE, in the glass substrate with a depth of 5 $\mu \mathrm{m}$. In a final step glass and silicon substrates were bonded via an anodic 
bonding process with a potential of $800 \mathrm{~V}$ and a temperature of $\sim 400$ ${ }^{\circ} \mathrm{C}$ applied. The spacing between the electrodes and nearest microfluidic channel wall is $15 \mu \mathrm{m}$. The final resulting structure is shown in Figure 6.9.
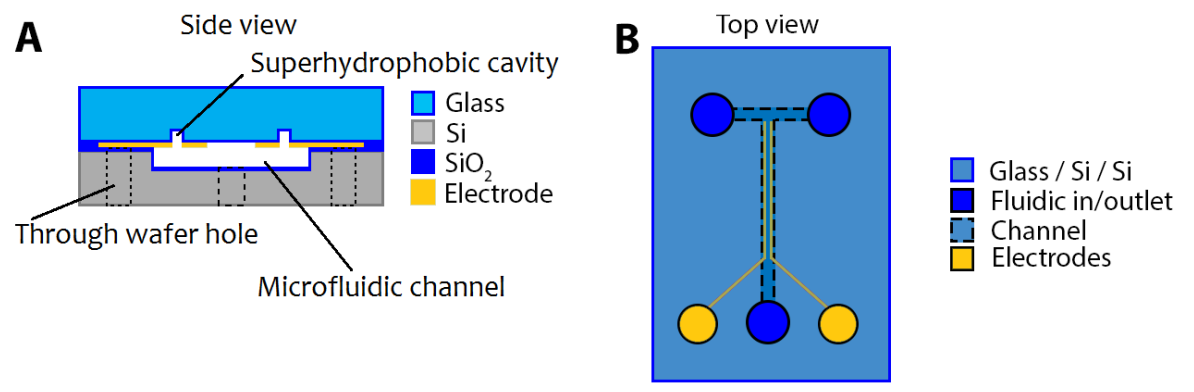

Figure 6.9: A) Side view of the sample is shown, with in light blue the glass wafer, in gray the silicon, dark blue the silicon oxide, and in red the electrodes. The cross sectional view shows the superhydrophobic cavity in the glass substrate. B) Topview of the resulting microfluidic cell. The fluidic in- and outlets are shown in dark blue, red shows the electrodes and the contact pads for electrical connection.

\section{Materials and setup}

Using optical microscopy and fluorescence microscopy the bubble evolution is imaged. Figure 6.10 shows a schematic drawing of the setup. The camera records via the fluorescent microscope (Zeiss Axio Zoom V16 with GFP filter excitation wavelength $470 \mathrm{~nm}$, emission $525 \mathrm{~nm}$ ), providing a top view of the sample. A pump (Elveflow OB1Mk3 and Harvard PHD Ultra Syringe Pump Infuse Only Standard) induces a flow through the microfluidic channel, where the liquid at the outlets is collected in closed containers. At the bottom side of the holder, an electrical contact is made to the electrodes inside the microfluidic channel using gold contact pins. A power supply (VSP-300 - Bio-Logic) is used to apply a constant current.

As fluorescent dye, sodium fluorescein salt is used which has a fluorescent response in electrolytes between $\mathrm{pH} 4$ and $\mathrm{pH} 10$ (here adjusted with $\mathrm{CH}_{3} \mathrm{COOH}$ or $\mathrm{NaOH}$ ), an excitation peak at 475-490 nm at pH 8.5, and an emission peak at $510-520 \mathrm{~nm}$ at $\mathrm{pH} 8.5$ [201]. The fluorescent response starts at $\mathrm{pH} 4$ and is increased almost linearly up to $\mathrm{pH} 8$ after which the emission was reported to remain constant up to $\mathrm{pH} 10$. 


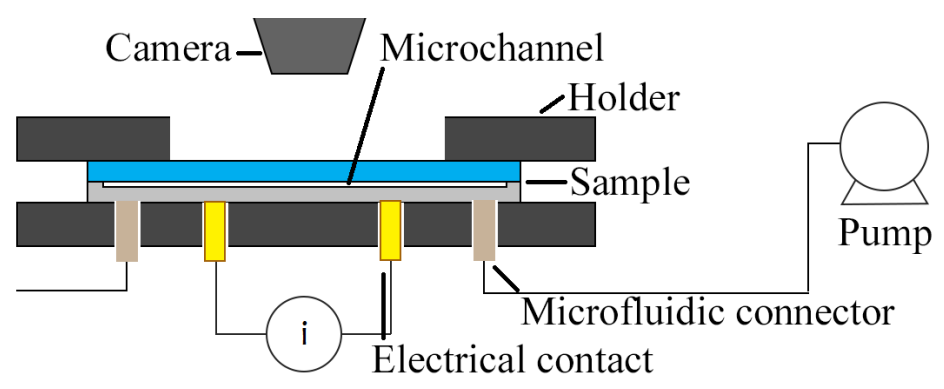

Figure 6.10: Schematic cross sectional view of the experimental setup. At the top the camera objective is shown, for fluorescence measurements a Zeiss Axio Zoom V16 microscope is used and for bright field optical microscopy a Flea3 camera was used. The holder (dark gray) contains the electrical contacts (yellow) and microfluidic connectors (light brown) as well as the sample. A flow is created using precision pumps (Elveflow OB1Mk3 and Harvard PHD Ultra Syringe Pump Infuse Only Standard). The electrolysis is carried out using a powersupply (VSP-300 - Bio-Logic).

\subsection{1 $\mathrm{pH}$ changes induced by gas evolution}

Figure 6.11 shows the fluorescent response of the electrolyte in the microfluidic channel with the gas evolving electrodes operating with various constant currents. The electrolyte contained $10.2 \mu \mathrm{M}$ of sodium fluorescein salt and $10 \mathrm{mM} \mathrm{KCl}$ had a $\mathrm{pH}$ of 5 and was kept at a constant flow rate of $50 \mu \mathrm{l} / \mathrm{min}$. At the anode (bottom electrode) the gas evolution resulted in the generation of protons leading to a lowered $\mathrm{pH}$ which translates into a darker fluorescent signal. The reaction of protons at the cathode (top electrode) increases the $\mathrm{pH}$ resulting in a fluorescent signal increase around the electrode. It can be seen that with increased current, the fluorescent response as a result of the $\mathrm{pH}$ change at the cathode increased, and decreased at the anode. At $100 \mu \mathrm{A}$ and $833 \mu \mathrm{A}$ bubble evolution is visible as well as the degree to which the boundary layers are affected. It should be noted that the bubble evolution occurs at the start of the electrodes where the length of the electrodes in series with the electrolyte is shortest.

Figure 6.12 shows the influence of flow on the fluorescent signal, a part of the microfluidic channel with the start of the electrodes is imaged. Water electrolysis with $1 \mathrm{~mA}$ constant current takes place with $10.2 \mu \mathrm{M}$ of sodium fluorescein salt and $10 \mathrm{mM} \mathrm{KCl} \mathrm{electrolyte} \mathrm{solution} \mathrm{(} \mathrm{pH}$ 5), for increasing flows from top to bottom $(0 \mu \mathrm{l} / \mathrm{hr}$ to $1.5 \mathrm{ml} / \mathrm{hr})$. The $\mathrm{pH}$ decrease at the anode during the electrolysis reaction decreases the electrolyte fluorescence signal. The resulting black region surrounding the anode has a front 


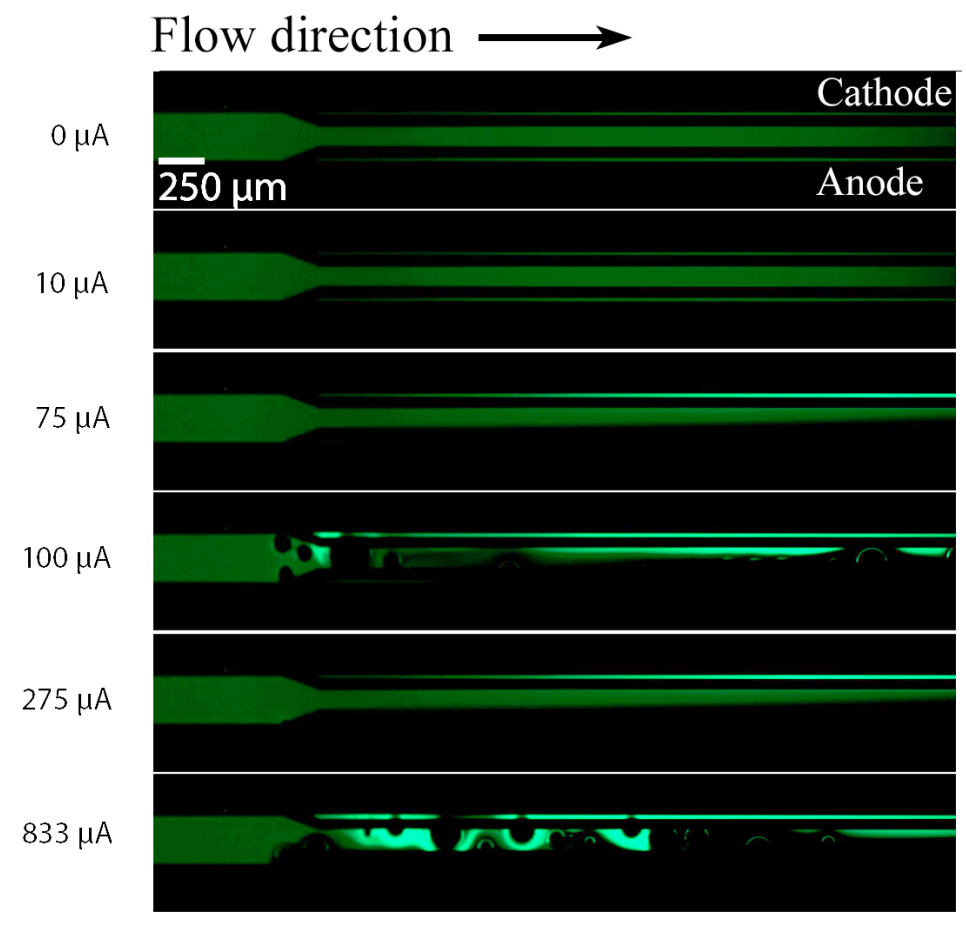

Figure 6.11: Fluorescent imaging of the fluorescent signal from electrolysis with sodium fluorescein salt present in the electrolyte. From top to bottom the constant current applied was $0 \mu \mathrm{A}, 10 \mu \mathrm{A}, 75 \mu \mathrm{A}, 100 \mu \mathrm{A}, 275 \mu \mathrm{A}$, and $833 \mu \mathrm{A}$. A constant flow rate of $50 \mu \mathrm{l} / \mathrm{min}$ was used for these measurements. The cathode consumes protons resulting in an increased $\mathrm{pH}$ and fluorescent signal, while at the anode protons are generated, lowering the $\mathrm{pH}$ resulting in a decreased fluorescent signal. The change in the fluorescent signal is visible for the applied currents of $75 \mu \mathrm{A}$ and above.

which is indicated by the white line. With the increased flow rate the formed species are transported away by the liquid in motion, resulting in a quenched fluorescent front.

Upon bubble evolution, the dissolved gas boundary layer is depleted from gas by bubbles taking in gas. Also, liquid convection occurs with the growth and detachment of bubbles resulting in a varying fluorescent signal as shown in Figure 6.13A. The fluorescent response of the $10.2 \mu \mathrm{M}$ sodium fluorescein salt in a $\mathrm{pH} 5$ solution (no flow conditions applied) is shown during bubble evolution on the cathode. Figure 6.13B shows the logarithmic radius of the bubble as function of the logarithmic time, the slope of 0.23 indicates that the bubble growth was not governed by the reaction nor diffusion. The coalescence of bubbles (small enough that no rapid or large changes in the measured bubble radius were observed at any 


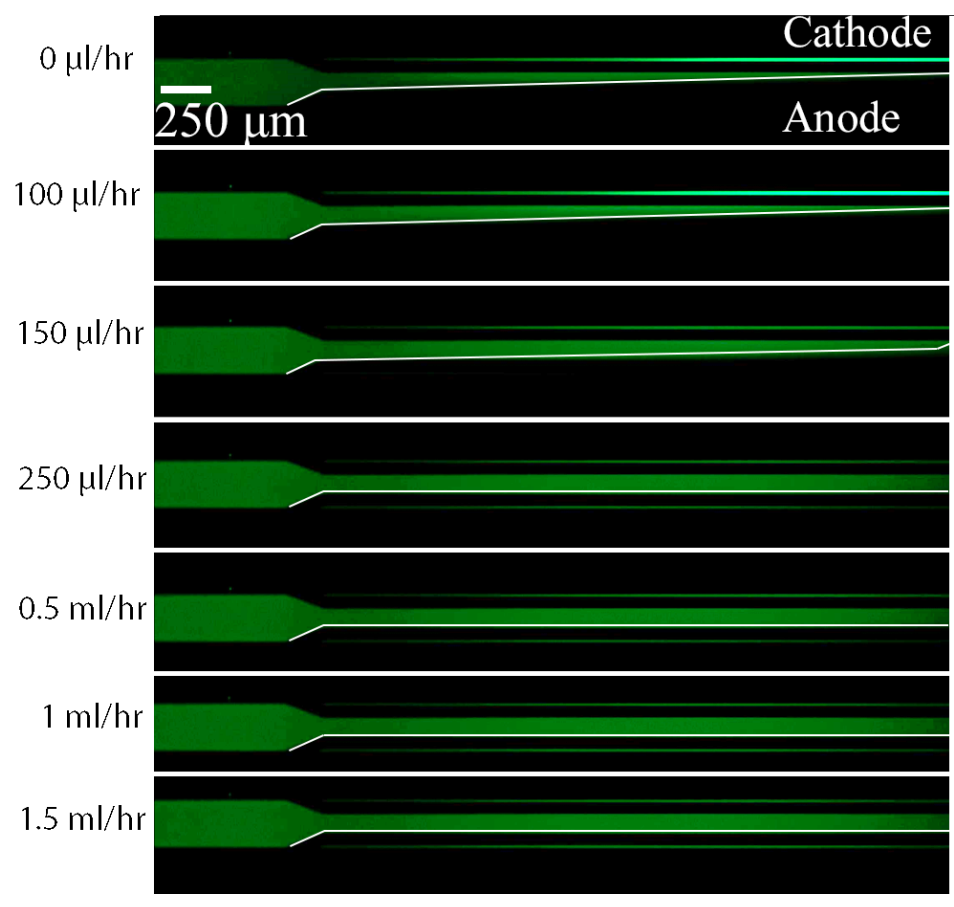

Figure 6.12: Fluorescent imaging of the $\mathrm{pH}$ responsive sodium fluorescein salt indicative for $\mathrm{pH}$ changes with product formation during electrolysis. The white line indicates the front of the fluorescent response to the product formation at the anode surface. From top to bottom a flow of $0 \mu \mathrm{l} / \mathrm{hr}, 100$ $\mu \mathrm{l} / \mathrm{hr}, 150 \mu \mathrm{l} / \mathrm{hr}, 250 \mu \mathrm{l} / \mathrm{hr}, 0.5 \mathrm{ml} / \mathrm{hr}, 1 \mathrm{ml} / \mathrm{hr}$, and $1.5 \mathrm{ml} / \mathrm{hr}$ was applied. With increased flow rate, the evolution front is more confined to the anode area in the channel.

instant) at the neck of the bubble might result in the enhanced growth rate. However, with a top view this could not be verified.

Although the membraneless electrolyzer functioned properly, the superhydrophobic cavities etched in the glass did not. No bubble formation was observed at the artificial nucleation sites. It was found that the fabrication process of the cavities in glass was not optimal. Instead of forming the desired smooth cylindrical shape during the dry etching process step, a rough tapered structure was formed with a wider opening than the bottom. Figure 6.14 shows a scanning electron microscopy image of the formed cavity. The tapered shape can lead to easier filling of the cavity by the electrolyte flow since the tapered angle might result in the air pocket moving out easier. Additionally, the increased pressure in the microfluidic channel might aid in filling the cavity with water [202]. The pressure increase combined with the decrease in supersaturation with the continuous 

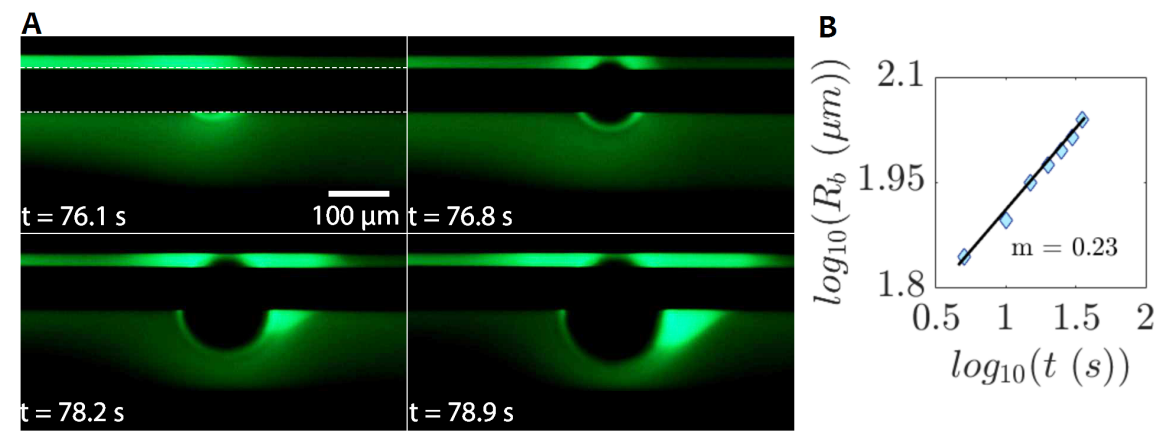

Figure 6.13: Fluorescence imaging of bubble evolution and tracking over time. A) The fluorescent response shows the $\mathrm{pH}$ increase at the cathode (between the dashed lines in the top left image). With increased time the boundary layer fluctuates with the growth of the bubble (black circle) on the electrode. B) The logarithmic radius of the bubble is set out with blue diamond markers against the logarithmic time, a slope $\mathrm{m}=0.23$ is found.

flow transporting products away down the microchannel, might lead to the cavities not acting as nucleation sites. However, filling the microchannel under low flow conditions and electrolysis without flow did not result in the superhydrophobic cavities functioning as intended either. Optimization of the deep reactive ion etching step in the glass wafer could improve the cylindrical shape which might provide a better functionality.

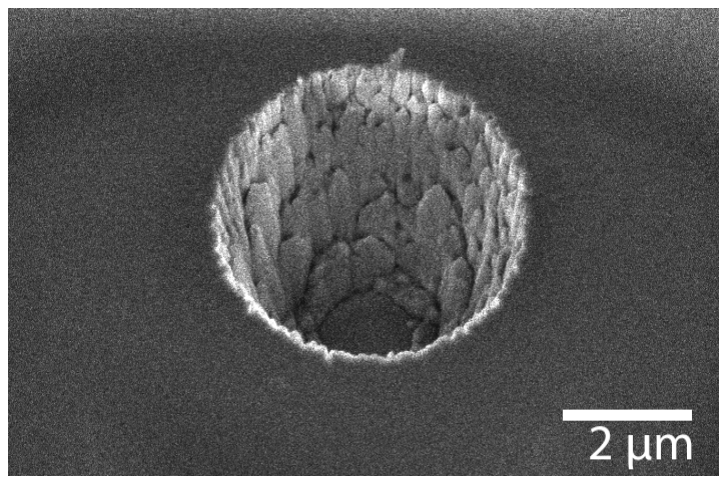

Figure 6.14: Scanning electron microscope image of a superhydrophobic cavity dry etching into a glass SCHOTT MEMpax wafer. 


\subsubsection{Preliminary conclusions}

The superhydrophobic cavities etched in glass did not function as artificial nucleation sites. The cavities were found to be non-cylindrical (tapered at the bottom) and rough. The cavity shape and pressure drop in the microfluidic channel might have lead to easier wetting of the cavities by the liquid. Fluorescent measurements with the membraneless electrolyzer, during electrolysis, showed $\mathrm{pH}$ fluctuations around the gas evolving electrodes due to the reaction and generation of protons at the cathode and anode, respectively. The fluorescence measurements showed that with flow, the boundary layer was spatially confined to the respective electrodes due to the introduced Poiseuille flow effectively move the gradient in $\mathrm{pH}$ to the sides of the microchannel. It was shown that the fluorescence signal was affected by the presence of bubbles on the electrodes, a result of the bubbles actively taking up gas and displacing the liquid.

\section{Acknowledgments}

The following people are thanked for the fruitful discussions on the subjects presented here: Zhixiao Zhao and Miguel Modestino. The people that helped out with the infrastructure of experiments or the experiments: Zhixiao Zhao, Stefan Schlautmann, Remco Sanders, Adlai Katzenberg, and Ben Rizkin are also thanked for their input. Furthermore, thanks to Stefan Schlautmann for the scanning electron microscopy image shown in Figure 6.14 .

Special thanks is given to the Modestino group at New York University - Tandon school for engineering for hosting part of the project and the Netherlands Center of Multiscale Catalysis (MCEC) for financing the project. 


\subsection{Final conclusions}

In this thesis we have investigated electrolytic bubbles and the phenomena associated to bubble evolution. Successive bubbles were studied by using artificial nucleation sites micromachined in, and near electrodes. Isolated bubbles were studied by spatially controlling the bubble evolution during electrolysis and pressure driven oversaturation.

Changes in dissolved gas boundary layer concentration influenced the bubble evolution, a decrease in gas concentration increases the nucleation time and slow down the diffusive growth, whilst an increase in gas concentration would exhibit the opposite behaviour. Hydrogen bubble evolution during electrolysis with silicon electrodes (that had a circular area with a radius of $3.5 \mathrm{~mm}$ in contact with the electrolyte) showed that the gas boundary layer initially evolved, but was depleted from gas by the formed bubbles. Upon continued reaction the gas boundary layer would counter the gas depletion by previously detached bubbles, resulting in subsequent faster bubble growth. The growth of bubbles was followed by detachment when the buoyancy force overcame the interfacial tension force, or when coalescence of two or more bubbles occurred. When the bubble contact line was pinned to the artificial nucleation site, the detachment size of bubbles was fixed by the maximum interfacial tension force determined by the circumference of the cavity. The controlled bubble evolution allowed the study of the influence of the gas boundary layer on successive isolated bubbles in detail.

Placement of artificial nucleation sites near electrodes resulted in the electrodes remaining free of bubbles within a certain operational regime of the current density. The controlled bubble evolution further allowed to study the electric response created by the bubbles near electrodes used in electrolysis of water. By forming the bubbles near electrodes, the screening effects by bubbles when formed on an electrode were decoupled from the concentration overpotential changes. It was found that the bubble detachment influenced the gas and proton concentration at the electrode surface, likely via induced convection of the liquid, resulting in a change in overpotential.

Measured bubble detachment radii varied, a possible result of contact line movement or contact line pinning to defects created during micromachining of the cavities. Further studies using artificial nucleation sites in electrolysis could investigate this in more detail by alternative micromachining steps limiting the possible causes. Furthermore, novel electrode designs incorporating artificial nucleation sites could allow for larger electrode surfaces to remain free of bubbles at higher current densities and warrants further investigation. 


\section{7}

\section{Bibliography}

[1] S. Shafiee and E. Topal. When will fossil fuel reserves be diminished? Energy Policy, 37:181-189, 2009.

[2] N. S. Lewis and D. G. Nocera. Powering the planet: Chemical challenges in solar energy utilization. Proc. Natl. Acad. Sci. U.S.A., 103:15729-15735, 2006.

[3] M. Grätzel. Photovoltaic and photoelectrochemical conversion of solar energy. Philos. Trans. Royal Soc. A, 365:993-1005, 2007.

[4] M. Grätzel. Recent advances in sensitized mesoscopic solar cells. Acc. Chem. Res., 42:1788-1798, 2009.

[5] P. Zhang, T. Wang, X. Chang, and J. Gong. Effective charge carrier utilization in photocatalytic conversions. Acc. Chem. Res., 49:911-921, 2016.

[6] S. Ardo, D. Fernandez Rivas, M. A. Modestino, V. Schulze Greiving, F. F. Abdi, E. Alarcon Llado, V. Artero, K. Ayers, C. Battaglia, J-P. Becker, D. Bederak, A. Berger, F. Buda, E. Chinello, B. Dam, V. Di Palma, T. Edvinsson, K. Fujii, H. Gardeniers, H. Geerlings, S. M. H. Hashemi, S. Haussener, F. Houle, J. Huskens, B. D. James, K. Konrad, A. Kudo, P. P. Kunturu, D. Lohse, B. Mei, E. L. Miller, 
G. F. Moore, J. Muller, K. L. Orchard, T. E. Rosser, F. H. Saadi, J-W. Schüttauf, B. Seger, S. W. Sheehan, W. A. Smith, J. Spurgeon, M. H. Tang, R. van de Krol, P. C. K. Vesborg, and P. Westerik. Pathways to electrochemical solar-hydrogen technologies. Energy Environ. Sci., 11:2768-2783, 2018.

[7] J. G. Canadell, C. Le Queéré, M. R. Raupach, C. B. Field, E. T. Buitenhuis, P. Ciais, T. J. Conway, N. P. Gillett, R. A. Houghton, and G. Marland. Contributions to accelerating atmospheric $\mathrm{CO}_{2}$ growth from economic activity, carbon intensity, and efficiency of natural sinks. Proc. Natl. Acad. Sci. U.S.A., 104:18866-18870, 2007.

[8] A. Fujishima and K. Honda. Electrochemical photolysis of water at a semiconductor electrode. Nature, 238:37-38, 1972.

[9] F. P. Bowden and K. E. W. Grew. Overpotential at very low current densities. The deposition of hydrogen from aqueous and nonaqueous electrolytes. Discuss. Faraday Soc., 1:86-90, 1947.

[10] R. de Levie. The electrolysis of water. J. Electroanal. Chem., 476:92-93, 1999.

[11] W. Ostwald. Elektrochemie, Ihre Geschichte un Lehre. Verlag von Veit \& Comp., Leipzig, 1896.

[12] R. G. Ehl and A. J. Ihde. Faraday's electrochemical laws and the determination of equivalent weights. J. Chem. Educ., 31:226, 1954.

[13] S. F. Jones, G. M. Evans, and K. P. Galvin. Bubble nucleation from gas cavities - a review. Adv. Coll. Interf. Sci, 80:27-50, 1999.

[14] P. S. Epstein and M. S. Plesset. On the stability of gas bubbles in liquid-gas solutions. J. Chem. Phys., 18:1505-1509, 1950.

[15] R. Kumar and N. K. Kuloor. The formation of bubbles and drops. Adv. Chem. Eng., 8:255-368, 1970.

[16] M. L. Storey, R. A. Forshee, and P. A. Anderson. Beverage consumption in the us population. J. Am. Diet Assoc., 106(12):1992-2000, 2006.

[17] A. Taqieddin, R. Nazari, L. Rajic, and A. Alshawabkeh. Review-physicochemical hydrodynamics of gas bubbles in two phase electrochemical systems. J. Electrochem. Soc., 194:E448-E459, 2017. 
[18] F. J. Montes, M. A. Galan, and R. L. Cerro. Mass transfer from oscillating bubbles in bioreactors. Chem. Eng. Sci., 54:3127-3136, 1999.

[19] S. M. Peyghambarzadeh, A. Vatani, and M. Jamialahmadi. Influences of bubble formation on different types of heat exchanger fouling. App. Therm. Eng., 50:848-856, 2013.

[20] N. S. Wang, J. D. Yang, R. V. Calabrese, and K. C. Chang. Unified modeling framework of cell death due to bubbles in agitated and sparged bioreactors. J. Biotechnol., 33:107-122, 1994.

[21] G. E. Thorncroft, J. F. Klausner, and R. Mei. An experimental investigation of bubble growth and detachment in vertical upflow and downflow boiling. Int. J. Heat Mass Transfer, 41:3857-3871, 1998.

[22] D. W. Dees and C. W. Tobias. Mass transfer at gas evolving surfaces. J. Electrochem. Soc., 134:1702-1713, 1987.

[23] X. Yang, F. Karnbach, M. Uhlemann, S. Odenbach, and K. Eckert. Dynamics of single hydrogen bubbles at a platinum microelectrode. Langmuir, 31:8184-8193, 2015.

[24] H. Vogt. The quantities affecting the bubble coverage of gas-evolving electrodes. Electrochim. Acta, 235:495-499, 2017.

[25] R. J. Thorne, C. Sommerseth, A. P. Ratvik, S. Rørvik, E. Sandnes, L. P. Lossius, H. Linga, and A. M. Svensson. Bubble evolution and anode surface properties in aluminium electrolysis. J. Electrochem. Soc., 162(8):E104-E114, 2015.

[26] Y. Kiros and B. Martin. Low energy consumption in chlor-alkali cells using oxygen reduction electrodes. Int. J. Electrochem. Sci., 3:444-451, 2008.

[27] P. van der Linde, P. Peñas López, Á. Moreno Soto, D. van der Meer, D. Lohse, H. Gardeniers, and D. Fernández Rivas. Gas bubble evolution on microstructured silicon substrates. Energy Environ. Sci., 11:3452 - 3462, 2018.

[28] P. van der Linde, Á. Moreno Soto, P. Peñas López, J. Rodríguez-Rodríguez, D. Lohse, J. G. E. Gardeniers, D. van der Meer, and D. Fernández Rivas. Electrolysis-driven and pressure-controlled diffusive growth of successive bubbles on microstructured surfaces. Langmuir, 33(45):12873-12886, 2017. 
[29] T. F. Groß, J. Bauer, G. Ludwig, D. Fernandez Rivas, and P. F. Pelz. Bubble nucleation from micro-crevices in a shear flow. Exp. Fluids, 59(12):12, 1-10, 2018.

[30] R. Leonard. A new oil world: The game has changed, but how? Africa is becoming a major new player in the new world hydrocarbon order. Am. For. Pol. Int., 35:352-359, 2013.

[31] J. Mayer, R.F. Sachesenhoger, C. Ungureanu, A. Bechtel, R. Gratzer, M. Sweda, and G. Tari. Petroleum chage and migration in the black sea: insight from oil and source rock geochemistry. J. Pet. Geo., 41:337-350, 2018.

[32] P. M. Crawford and J. C. Killen. New challenges and directions in oil shale development technologies. ACS Sym. Series, 1032:21-60, 2010.

[33] R. W. Bentley. Global oil \& gas depletion: an overview. Energy Policy, 7:189-205, 2002.

[34] P. Denholm and R. M. Margolis. Evaluating the limits of solar photovoltacis (PV) in electric power systems utilizing energy storage and other enabling technologies. Energy Policy, 35:4424-4433, 2007.

[35] A. Züttel, A. Remhof, A. Borgschulte, and O. Friedrichs. Hydrogen: the future energy carrier. Philos. Trans. Royal Soc. A, 368:3329-3342, 2010.

[36] G. A. Olah. Beyond oil and gas: The methanol economy. Angewandte Chemie, 44:2636-2639, 2005.

[37] B. Dunn, H. Kamath, and J.-M. Tarascon. Electrical energy storage for the grid: A battery of choices. Science, 334:928-935, 2011.

[38] G. W. Crabtree and M. S. Dresselhaus. The hydrogen fuel alternative. MRS Bull, 33:421-428, 2008.

[39] R. Krishna, E. Titus, M. Salimian, O. Okhay, S. Rajendran, A. Rajkumar, J. M. G. Sousa, A. L. C. Ferreira, J. C. Gil, and J. Gracio. Hydrogen Storage. Intech, London, 2012.

[40] A. Züttel. Hydrogen storage methods. Naturwissenschaften, 91:157-172, 2004.

[41] B. Sakintuna, F. Lamari-Darkrim, and M. Hirscher. Metal hydride materials for solid hydrogen storage: A review. Int. J. Hydrogen Energy, 32:1121-1140, 2007. 
[42] J. W. Sheffield, K. B. Martin, and R. Folkson. In Alternative Fuels and Advanced Vehicle Technologies for Improved Environmental Performance, chapter Electricity and hydrogen as energy vectors for transportation vehicles, pages 117-137. Woodhead Publishing, 2014.

[43] BP. BP statiscal review of world energy. https://www.bp. com/content/dam/bp/en/corporate/pdf/energy-economics/ statistical-review/bp-stats-review-2018-full-report.pdf, 2018. Accessed: 2018-07-31.

[44] M. R. Shaner, H. A. Atwater, N. S. Lewis, and E. W. McFarland. A comparative technoeconomic analysis of renewable hydrogen production using solar energy. Energy Environ. Sci., 9:2354-2371, 2016.

[45] F. Yilmaz, M. T. Balta, and R. Selbaș. A review of solar based hydrogen prodruction methods. Ren. Sust. Energy Rev., 56:171-178, 2016.

[46] M. A. Modestino, D. Fernandez Rivas, S. M. H. Hashemi, J. G. E. Gardeniers, and D. Psaltis. The potential for microfluidics in electrochemical energy systems. Energy Environ. Sci., 9:3381-3391, 2016.

[47] G. Planinšič and M. Vollmer. The surface-to-volume ratio in thermal physics from cheese cube physics to animal metabolism. Eur. J. Phys., 29:369-384, 2008.

[48] S. Dutta. Technology assessment of advanced electrolytic hydrogen production. Int. J. Hydrogen Energy, 15:379-386, 1990.

[49] K. Mazloomi, N. B. Sulaiman, and H. Moayedi. Electrical efficiency of electrolysis hydrogen production. Int. J. Electrochem. Sci., 7:3314-3326, 2012.

[50] E. L. Cussler. Diffusion: Mass Transfer in Fluid Systems. Cambridge University Press, $2^{\text {nd }}$ edition, 1997.

[51] N. Nagai, M. Takeuchi, T. Kimura, and T. Oka. Existence of optimum space between electrodes on hydrogen production by water electrolysis. Int. J. Hydrogen Energy, 28:3314-3326, 2003.

[52] D. A. Noren and M. A. Hoffman. Clarifying the Butler-Volmer equation and related approximations for calculating activation losses in solid oxide fuel cell models. J. Power Sources, 152:175-181, 2005. 
[53] C. Xiang, K. M. Papadantonakis, and N. S. Lewis. Principles and implementations of electrolysis systems for water splitting. Mater. Horiz., 3:169-173, 2016.

[54] S. Cherevko, S. Geiger, O. Kasian, N. Kulyk, J-P. Grote, A. Savan, B. R. Shrestha, B. Merzlikin, S. Breitbach, and K. J. J. Ludwig, A. Mayrhofer. Oxygen and hydrogen evolution reactions on $\mathrm{Ru}$, $\mathrm{RuO}_{2}$, Ir, and $\mathrm{IrO}_{2}$ thin film electrodes in acidic and alkaline electrolytes: A comparative study on activity and stability. Catalysis Today, 262:170-180, 2016.

[55] M. W. Louie and A. T. Bell. A investigation of thin-film Ni-Fe oxide catalysts for the electrochemical evolution of oxygen. J. Am. Chem. Soc., 135:12329-12337, 2013.

[56] K. Zeng and D. Zhang. Recent progress in alkaline water electrolysis for hydrogen production and applications. Prog. Energy Combust. Sci., 36:307-326, 2010.

[57] P. Quaino, F. Juarez, E. Santos, and W. Schmickler. Volcano plots in hydrogen electrocatalysis - uses and abuses. Beilstein J. Nanotechnol., 5:846-854, 2014.

[58] M. G. Walter, E. L. Warren, S. W. McKone, J. R. Boettcher, Q. Mi, E. A. Santori, and N. S. Lewis. Solar water splitting cells. Chem. Rev., 110:6446-6473, 2010.

[59] A. Kudo and Y. Miseko. Heterogeneous photocatalyst materials for water splitting. Chem. Soc. Rev., 38:253-278, 2009.

[60] B. Bensmann, R. Hanke-Rauschenbach, and K. Sundmacher. In-situ measurement of hydrogen crossover in polymer electrolyte membrane water electrolysis. Int. J. Hydrogen Energy, 39:49-53, 2014.

[61] M. J. Lavorante and J. I. Reynoso, C. Y. Franco. Water electrolysis with Zirfon $\AA$ as separator and $\mathrm{NaOH}$ as electrolyte. Desalin. Water Treat., 56:3647-3653, 2014.

[62] C. Heitner-Wirguin. Recent advances in perfluorinated ionomer membranes: structure, properties and applications. J. Membrane Sci., 120:1-33, 1996.

[63] Q. Feng, X-Z. Yuan, G. Liu, B. Wei, Z. Zhang, H. Li, and H. Wang. A review of proton exchange membrane water electrolysis on degradation mechanisms and mitigation strategies. J. Power Sources, 366:33-55, 2017. 
[64] M. Paidar, V. Fateev, and K. Bouzek. Membrane electrolysis - history, current status and perspective. Electrochim. Acta, 209:737-756, 2016.

[65] J. T. Davis, J. Qi, X. Fan, J. C. Cui, and D. V. Esposito. Floating membraneless $\mathrm{PV}$-electrolyzer based on buoyancy-driven product separation. Int. J. Hydrogen Energy, 43:1224-1238, 2018.

[66] S. M. H. Hashemi, M. A. Modestino, and D. Psaltis. A membrane-less electrolyzer for hydrogen production across the $\mathrm{pH}$ scale. Energy Environ. Sci., , 8:2003-2009, 2015.

[67] C. W. Tobias. Effect of gas evolution on current distribution and ohmic resistance in electrolyzers. J. Electrochem. Soc., 106:833-838, 1959.

[68] R. E. De La Rue and C. W. Tobias. On the conductivity of dispersions. J. Electrochem. Soc., 106:827-833, 1959.

[69] J. Dukovic and C. W. Tobias. The influence of attached bubbles on potential drop and current distribution at gas-evolving electrodes. $J$. Electrochem. Soc., 134(2):2, 331-343, 1987.

[70] C. Gabrielli, F. Huet, and R. P. Noqueira. Fluctuations of concentration overpotential generated at gas-evolving electrodes. Electrochim. Acta, 50:3726-3736, 2005.

[71] F. Hine and K. Murakami. Bubble effects on the solution IR drop in a vertical electrolyzer under free and forced convection. J. Electrochem. Soc., 127:292-297, 1980.

[72] L. J. J. Jansen and G. J. Visser. Distribution of void fraction, ohmic resistance and current in a tall vertical gas-evolving cell. Proc. Electrochem. Cell Des. Optim., 123:361-385, 1990.

[73] H. Vogt. A hydrodynamic model for the ohmic interelectrode resistance of cells with vertical gas evolving electrodes. Electrochim. Acta, 26:1311-1317, 1981.

[74] P. J. Sides and C. W. Tobias. Primary potential and current distribution around a bubble on an electrode. J. Electrochem. Soc., 127:288-291, 1980.

[75] R. L. LeRoy. Analysis of time-variation effects in water electrolyzers. J. Electrochem. Soc., 126:1674-1682, 1979. 
[76] B. E. Bongenaar-Schlenter, L. J. J. Janssen, S. J. D. van Stralen, and $\mathrm{E}$. Barendrecht. The effect of the gas void distribution on the ohmic resistance during water electrolytes. J. Appl. Electrochem., 15:537-548, 1985.

[77] P. J. Sides and C. W. Tobias. Resistance of a planar array of spheres: Gas bubbles on an electrode. J. Electrochem. Soc., 129:827-833, 1982.

[78] A. Volanschi, W. Olthuis, and P. Bergveld. Gas bubbles electrolytically generated at microcavity electrodes (MCE) used for the measurement of the dynamic surface tension in liquids. Sens. Actuators A Phys., 52:18-22, 1996.

[79] A. Volanschi, J. G. H. Nijman, W. Olthuis, and P. Bergveld. Microcavity electrodes used as single-nucleation site electrodes for the electrolysis of water. Sens. Mat., 9:223-240, 1997.

[80] K. W. Kolasinski. Bubbles: A review of their relationship to the formation of thin films and porous materials. Mesoporous Biomater., 1:49-60, 2014.

[81] P. M. Wilt. Nucleation rates and bubble stability in water-carbondioxide solutions. J. Colloid Interface Sci., 112:530-538, 1986.

[82] S. Lubetkin and M. Blackwell. The nucleation of bubbles in supersaturated solutions. J. Colloid Interface Sci., 126:610-615, 1988.

[83] H. J. Maris. Introduction to the physics of nucleation. C. R. Physicique, 7:946-958, 2006.

[84] Y. Liu and S. J. Dillon. In situ observation of electrolytic $\mathrm{H}_{2}$ evolution adjacent to gold cathodes. Chem. Commun., 50:1761-1763, 2014.

[85] D. V. Dapkus and P. J. Sides. Nucleation of electrolytically evolved hydrogen at an idealy smooth electrode. J. Colloid Interface Sci., 111:133-151, 1986.

[86] S. R. German, M. A. Edwards, Q. Chen, Y. Liu, L. Luo, and H. S. White. Electrochemistry of single nanobubbles. Estimating the critical size of bubbleforming nuclei for gas-evolving electrode reactions. Faraday Discuss., 193:223-240, 2016. 
[87] C. A. Ward, A. Blakrishnan, and F. C. Hooper. On the thermodynamics of nucleation in weak gas-liquid solutions. J. Basic Eng., 92:695-701, 1970.

[88] S. D. Lubetkin. Why is it much easier to nucleate gas bubbles than theory predicts? Langmuir, 19:2575-2587, 2003.

[89] C. A. C. Sequeira, D. M. F. Santos, B. Sljukic, and L. Amaral. Physics of electrolytic gas evolution. Braz. J. Phys., 43:199-208, 2013.

[90] P. G. Bowers, K. Bar-Eli, and R. M. Noyes. Unstable supersaturation solutions of gases in liquids and nucleation theory. J. Chem. Soc. Faraday Trans., 92:2843-2849, 1996.

[91] N. P. Brandon, G. H. Kelsall, S. Levine, and A. L. Smith. Interfacial electrical properties of electrogenerated bubbles. Electrophoresis, 15:485-493, 1985.

[92] N. P. Brandon and G. H. Kelsall. Growth kinetics of bubbles electrogenerated at microelectrodes. J. Appl. Electrochem., 15:475-484, 1985.

[93] D. Fernández, P. Maurer, M. Marine, J. M. D. Coey, and M. E. Möbius. Bubble formation at a gas-evolving microelectrode. Langmuir, 30:13065-13074, 2014.

[94] Q. Chen, L. Luo, and H. S. White. Electrochemical generation of a hydrogen bubble at a recessed platinum nanopore electrode. Langmuir, 31:4573-4581, 2015.

[95] Z. Hammadi, R. Morin, and J. Olives. Field nano-localization of gas bubble production from water electrolysis. Appl. Phys. Let., 103:223106, 2013.

[96] R. Darby and M. S. Haque. The dynamics of electrolytic hydrogen bubble evolution. Chem. Eng. Sci., 28:1129-1138, 1973.

[97] C. Brussieux, P. Viers, H. Roustan, and M. Rakib. Controlled electrochemical gas bubble release from electrodes entirely and partially covered with hydrophobic materials. Electrochim. Acta, 56:7194-7201, 2011.

[98] T. Kadyk, D. Bruce, and M. Eikerling. How to enhance gas removal from porous electrodes? Sci. Rep., 6:38780, 2016.

[99] D. E. Westerheide and J. W. Westwater. Isothermal growth of hydrogen bubbles during electrolysis. A.I.Ch.E., 7:357-362, 1961. 
[100] Y. Wang, X. Hu, Z. Cao, and L. Guo. Investigations on bubble growth mechanism during photoelectrochemical and electrochemical conversions. Colloids Surf., A, 505:86-92, 2016. $6^{\text {th }}$ International Workshop on Bubble and Drop Interfaces.

[101] L. E. Scriven. On the dynamics of phase growth. Chem. Eng. Sci., 10:1-13, 1959.

[102] H. K. Forster and N. Zuber. Dynamics of vapor bubbles and boiling heat transfer. AIChE J., 1:531-535, 1955.

[103] Á. Moreno Soto, A. Prosperetti, D. Lohse, and D. van der Meer. Gas depletion through single gas bubble diffusive growth and its effect on subsequent bubbles. J. Fluid Mech., 831:474-490, 2017.

[104] H. F. A. Verhaart, R. M. de Jonge, and S. J. D. van Stralen. Growth rate of gas bubble during electrolysis in supersaturated liquid. Int. $J$. Heat Mass Transfer, 23:293-299, 1980.

[105] B. C. Donose, F. Harnish, and E. Taran. Electrochemically produced hydrogen bubble probes for gas evolution kinetics and force spectrocopy. Electrochem. Comm., 24:21-24, 2012.

[106] F. Yang, M. Manjare, Y. Zhao, and R. Qiao. On the peculiar bubble formation, growth, and collapse behaviors in catalytic micro-motor systems. Microfluid. Nanofluid., 21:6, 2017.

[107] H. N. Og̃uz and A. Prosperetti. Dynamics of bubble growth and detachment from a needle. J. Fluid Mech., 257:111-145, 1993.

[108] S. Lee, W. Sutomo, C. Liu, and E. Loth. Micro-fabricated electrolytic micro-bubblers. Int. J. Multiphase Flow, 31:706-722, 2005.

[109] H. Vogt, Ö. Aras, and R. J. Balzer. The limits of the analogy between boiling and gas evolution at electrodes. Int. J. Heat Mass Trans., 47:787-795, 2004.

[110] P. Hanafizadeh, J. Eshraghi, E. Kosari, and W. H. Ahmed. The effect of gas properties on bubble formation, growth, and detachment. Part. Sci. Tech., 33:645-651, 2015.

[111] S. Di Bari and A. Robinson. Experimental study of gas injected bubble growth from submerged orifices. Exp. Therm. Fluid Sci., 44:124-137, 2013.

[112] G. Duhar and C. Colin. Dynamics of bubble growth and detachment in a viscous shear flow. Phys. Fluids, 18:077101, 2006. 
[113] O. R. Enríquez, C. Sun, D. Lohse, A. Prosperetti, and D. van der Meer. The quasi-static growth of $\mathrm{CO}_{2}$ bubbles. J. Fluid Mech., 741(R1), 2014.

[114] W. Fritz. Berechnung des maximal volume von dampfblasen. Phys. Z., 36:379-388, 1935.

[115] S. J. D. van Stralen and R. Cole. Boiling phenomena Vol. 1. Hemisphere, $1^{\text {st }}$ edition, 1979.

[116] D. Zhang and K. Zeng. Evaluating the behavior of electrolytic gas bubbles and their effect on the cell voltage in alkaline water electrolysis. Ind. Eng. Chem. Res., 51:13825-13832, 2012.

[117] H. Vogt. Superposition of microconvective and macroconvective mass transfer at gas-evolving electrodes - a theoretical attempt. Electrochim. Acta, 32:633-636, 1987.

[118] H. K. Nahra and Y. Kamotani. Prediction of bubble diameter at detachment from a wall orifice in liquid cross-flow under reduced and normal gravity conditions. Chem. Eng. Sci., 58:55-69, 2003.

[119] V. G. Nefedov, V. M. Serebritskii, and E. A. Borshchenko. Electrolysis of water at high electric field intensities: The fountain effects. Russ. J. Electrochem., 34(9):852-856, 1998.

[120] R. Massoudi and A. D. King. Effect of pressure on the surface tension of water. Adsorption of low molecular weigth gases on water at $25^{\circ}$. J. Phys. Chem., 78:2262-2266, 1974.

[121] S. Jain and L. Qiao. Molecular dynamics simulations of the surface tension of oxygen-supersaturated water. AIP $a d v$., 7:045001, 2017.

[122] C. Marangoni. On the expansion of a drop of liquid floating on the surface of another liquid. Tipographia dei fratelli Fusi, Pavia, 1865.

[123] S. Lubetkin. The motion of electrolytic gas bubbles near electrodes. Electrochim. Acta, 48:357-375, 2002.

[124] X. Yang, D. Baczyzmalski, C. Cierpka, G. Mutschke, and K. Eckert. Marangoni convection at electrogenerated hydrogen bubbles. Phys. Chem. Chem. Phys., 20:11542-11548, 2018.

[125] X. Hu, Z. Cao, S. Wang, Y. Shen, L. Guo, and J. Chen. Single photogenerated bubble at gas-evolving $\mathrm{TiO}_{2}$ nanorod-array electrode. Electrochim. Acta, 202:175-185, 2016. 
[126] A. E. Dorfi, A. C. West, and D. V. Esposito. Quantifying losses in photoelectrode performance due to single hydrogen bubbles. J. Phys. Chem. C, 121:26587-26597, 2017.

[127] H. Matsushima, D. Kiuchi, and Y. Fukunaka. Measurement of dissolved hydrogen supersaturation during water electrolysis in a magnetic field. Electrochim. Acta, 54:5858-5862, 2009.

[128] M-Y. Lin, L-W. Hourng, and C-W. Kuo. The effect of magnetic on hydrogen production efficiency in water electrolysis. Int. J. Hydrogen Energy, 37:1311-1320, 2012.

[129] M-Y. Lin and L-W. Hourng. Effects of magnetic field and pulse potential on hydrogen production via water electrolysis. Int. J. Energy Res., 38:106-116, 2014.

[130] T. Iida, H. Matsushima, and Y. Fukunaka. Water electrolysis under a magnetic field. J. Electrochem. Soc., 154:E112-E115, 2007.

[131] J. A. Koza, S. Mühlenhoff, P. Źabiński, P. A. Nikrityuk, K. Eckert, M. Uhlemann, A. Gebert, T. Weier, L. Schultz, and S. Odenbach. Hydrogen evolution under the influence of a magnetic field. Electrochim. Acta, 56:2665-2675, 2011.

[132] H. Matsushima, T. Iida, and Y. Fukunaka. Gas bubble evolution on transparent electrode during water electrolysis in a magnetic field. Electrochim. Acta, 100:261-264, 2013.

[133] Z. Diao, P. A. Dunn, G. Zangari, and J. M. D. Coey. Electrochemical noise analysis of the effects of a magnetic field on cathodic hydrogen evolution. Electrochem. Comm., 11:740-743, 2009.

[134] M. H. Islam, O. S. Burheim, and B. G. Pollet. Sonochemical and sonoelectrochemical production of hydrogen. Ultrasonics Sonochemistry, 2018.

[135] S-D. Li, C-C. Wang, and C-Y. Chen. Water electrolysis in the presence of an ultrasonic field. Electrochim. Acta, 54:3877-3883, 2009.

[136] J. Li, J. Xue, Z. Tan, Y. Zheng, and L. Zhang. Ultrasound-Assisted Electrolysis in $\mathrm{NaOH}$ Solution for Hydrogen Generation, pages 919-926. John Wiley \& Sons, Ltd, 2012.

[137] L. Barelli, G. Bidini, F. Gallorini, and S. Servili. Hydrogen production through sorption-enhanced steam methane reforming and membrane technology: A review. Energy, 33(4):4 , 554-570, 2008. 
[138] S. A. Chattanathan, S. Adhikari, and N. Abdoulmoumine. A review on current status of hydrogen production from bio-oil. Renew. Sust. Energ. Rev., 16(5):5, 2366-2372, 2012.

[139] K. Maeda, T. Takata, M. Hara, N. Saito, Y. Inoue, H. Kobayashi, and K. Domen. GaN:ZnO solid solution as a photocatalyst for visible-light-driven overall water splitting. J. Am. Chem. Soc., 127:8286-8287, 2005.

[140] K. Maeda and K. Domen. Theoretical and computational chemistry, volume 18. Elsevier B. V., 2007.

[141] I. P. Jain. Hydrogen the fuel for 21st century. Int. J. Hydrogen Energy, 34(17):17, 7368-7378, 2009.

[142] S. Y. Reece, J. A. Hamel, K. Sung, T. D. Jarvi, A. J. Esswein, J. J. H. Pijpers, and D. G. Nocera. Wireless solar water splitting using silicon-based semiconductors and earth-abundant catalysts. Science, 334(6056):6056, 645-648, 2011.

[143] J. W. Westwater. Advances in chemical engineering, volume 2. Elsevier Inc., 1958.

[144] G. A. Somorjai and Y. Li. Introduction to surface chemistry and catalysis. John Willey and Sons, Inc., Hoboken, New Jersey, $2^{\text {nd }}$ edition, 2010.

[145] Q. Chen, L. Luo, H. Faraji, S. W. Feldberg, and H. S. White. Electrochemical measurements of single $\mathrm{H}_{2}$ nanobubble nucleation and stability at Pt nanoelectrodes. J. Phys. Chem. Lett., 5(20):20, 3539-3544, 2014.

[146] P. Lv, H. Le The, J. Eijkel, A. van den Berg, X. Zhang, and D. Lohse. Growth and detachment of oxygen bubbles induced by gold-catalyzed decomposition of hydrogen peroxide. J. Phys. Chem. C, 121:20769-20776, 2017.

[147] X. Zhang, A. Kumar, and P. J. Scales. Effects of solvency and interfacial nanobubbles on surface forces and bubble attachment at solid surfaces. Langmuir, 27(6):6, 2484-2491, 2011.

[148] C. W. M. P. Sillen. The effect of gas bubble evolution on the energy efficiency in water electrolysis. $\mathrm{PhD}$ thesis, Technische Universiteit Eindhoven, 1983. 
[149] J. Eigeldinger and H. Vogt. The bubble coverage of gas-evolving electrodes in a flowing electrolyte. Electrochim. Acta, 45(27):27, 4449-4456, 2000.

[150] N. Bremond, M. Arora, C.-D. Ohl, and D. Lohse. Controlled multibubble surface cavitation. Phys. Rev. Lett., 96(224501):1-4, 2006.

[151] D. Fernandez Rivas, A. Prosperetti, A. G. Zijlstra, D. Lohse, and H. J. G. E. Gardeniers. Efficient sonochemistry through microbubbles generated with micromachined surfaces. Angew. Chem. Int. Ed., 49(50):50, 9699-9701, 2010.

[152] D. Narezo Guzman, Y. Xie, S. Chen, D. Fernandez Rivas, C. Sun, D. Lohse, and G. Ahlers. Heat-flux enhancement by vapour-bubble nucleation in Rayleigh-Bénard turbulence. J. Fluid Mech., 787:331-366, 2016.

[153] O. R. Enríquez, C. Hummelink, G-W. Bruggert, D. Lohse, A. Prosperetti, D. van der Meer, and C. Sun. Growing bubbles in a slightly supersaturated liquid solution. Rev. Sci. Instrum., 84:065111, 2013.

[154] R. Elbersen, R. M. Tiggelaar, A. Milbrat, G. Mul, H. Gardeniers, and J. Huskens. Controlled doping methods for radial $\mathrm{p} / \mathrm{n}$ junctions in silicon. Adv. Energy Mater., 5(1401745):1401745, 1-8, 2015.

[155] D. V. Esposito, Y. Lee, H. Yoon, P. M. Haney, N. Y. Labrador, T. P. Moffat, A. A. Talin, and V. A. Szalai. Deconvoluting the influences of 3d structure on the performance of photoelectrodes for solar-driven water splitting. Sustain. Energ. Fuels, 1(154):154-173, 2017.

[156] B. M. Borkent, S. Gekle, A. Prosperetti, and D. Lohse. Nucleation threshold and deactivation mechanisms of nanoscopic cavitation nuclei. Phys. Fluids, 21(102003):1-9, 2009.

[157] W. Henry. Experiments on the quantity of gases absorbed by water, at different temperature, and under different pressures. Philos. Trans. Royal Soc. A, 93:29-42+247-275, 1803.

[158] A. S. Tucker and C. A. Ward. Critical state of bubbles in liquid-gas solutions. J. Appl. Phys., 46(11):4801-4808, 1975.

[159] M. E. Tawfik and F. J. Diez. On the relation between onset of bubble nucleation and gas supersaturation concentration. Electrochim. Acta, 146:792-797, 2014. 
[160] V. B. Svetovoy, R. G. P. Sanders, and M. C. Elwenspoek. Transient nanobubbles in short-time electrolysis. J. Phys.: Condens. Matter, 25(184002):184002, 1-12, 2013.

[161] R. H. Dettre and R. E. Johnson, Jr. Contact angle hysteresis, volume 69, chapter Contact angle hysteresis. IV. Contact angle measurements on heterogeneous surfaces, pages 136-144. Frederick M. Fowkes, 1965.

[162] P. G. De Gennes. Wetting: statics and dynamics. Rev. Mod. Phys., 57(3):3, 827-863, 1985.

[163] W. J. Park, J. H. Kim, S. M. Cho, S. G. Yoon, S. J. Suh, and D. H. Yoon. High aspect ratio via etching conditions for deep trench of silicon. Surf. Coat. Technol., 171:290-295, 2003.

[164] S. Ramos and A. Tanguy. Pinning-depinning of the contact line on nanorough surfaces. Eur. Phys. J. E, 19(4):4, 433-440, 2006.

[165] T. Baum, J. Satherley, and D. J. Schiffrin. Contact angle, gas bubble detachment, and surface roughness in the anisotropic dissolution of $\mathrm{Si}(100)$ in aqueous KOH. Langmuir, 14(10):10 , 2925-2928, 1998.

[166] G. S. Barker, B. Jefferson, and S. J. Judd. The control of bubble size in carbonated beverages. Chem. Eng. Sci., 57:565-573, 2002.

[167] M. Krasowska and K. Malysa. Kinetics of bubble collision and attachment to hydrophobic solids: I. Effect of surface roughness. Int. J. Miner. Process., 81:205-216, 2007.

[168] F. Tomasoni, H. van Parys, H. Terryn, A. Hubin, J. Deconinck, J.-M. Buchlin, and J. van Beeck. Identification of bubble evolution mechanisms during AC electrograining. Electrochem. Comm., 12:156-159, 2010.

[169] T. Nierhaus, H. van Parys, S. Dehaeck, J. van Beeck, H. Deconinck, J. Deconinck, and A. Hubin. Simulation of the two-phase flow hydrodynamics in an IRDE reactor. J. Electrochem. Soc., 156(9):P139-P148, 2009.

[170] L. Zhang, Y. Zhang, X. Zhang, Z. Li, G. Shen, M. Ye, C. Fan, H. Fang, and J. Hu. Electrochemically controlled formation and growth of hydrogen nanobubbles. Langmuir, 22(19):8109-8113, 2006.

[171] J. J. Chen, M. H. Liao, K. T. Li, and C. M. Shen. One-heater flow-through polymerase chain reaction device by heat pipes cooling. Biomicrofluidics, 9, 2015. 
[172] J. Nowotny, C. C. Sorrell, L. R. Sheppard, and T. Bak. Solar-hydrogen: Environmentally safe fuel for the future. Int. J. Hydrogen Energy, 30:521-544, 2005.

[173] J. P. Glas and J. W. Westwater. Measurements of the growth of electrolytic bubbles. Int. J. Heat Mass Transfer, 7:1427-1443, 1964.

[174] H. F. A. Verhaart, R. M. de Jonge, and S. J. D. van Stralen. Growth rate of a gas bubble during electrolysis in supersaturated liquid. Int. J. Heat Mass Transfer, 23:293-299, 1979.

[175] G. Sakuma, Y. Fukunaka, and H. Matsushima. Nucleation and growth of electrolytic gas bubbles under microgravity. Int. $J$. Hydrogen Energy, 39:7638-7645, 2014.

[176] P. Chandran, S. Bakshi, and D. Chatterjee. Study on the characteristics of hydrogen bubble formation and its transport during electrolysis of water. Chem. Eng. Sci., 138:99-109, 2015.

[177] A. J. Leenheer and H. A. Atwater. Water-splitting photoelectrolysis reaction rate via microscopic imaging of evolved oxygen bubbles. $J$. Electrochem. Soc., 157(9):B1290-B1294, 2010.

[178] Y. Wang, M. E. Zaytsev, H. Le The, J. C. T. Eijkel, H. J. W. Zandvliet, $\mathrm{X}$. Zhang, and D. Lohse. Vapor and gas-bubble growth dynamics around laser-irradiated, water-immersed plasmonic nanoparticles. ACS Nano, 11(2):2045-2051, 2017.

[179] H. Vogt. The rate of gas evolution of electrodes-I. An estimate of the efficiency of gas evolution from the supersaturation of electrolyte adjacent to a gas-evolving electrode. Electrochim. Acta, 29(2):167-173, 1984.

[180] D. Lohse and X. Zhang. Surface nanobubbles and nanodroplets. Rev. Mod. Phys., 87:981-1035, 2015.

[181] L. J. J. Janssen and J. G. Hoogland. The effect of electrolytically evolved gas bubbles on the thickness of the diffusion layer. Electrochim. Acta, 15:1013-1023, 1970.

[182] H. Vogt and K. Stephan. Local microprocesses at gas-evolving electrodes and their influence on mass transfer. Electrochim. Acta, 155:348-356, 2015.

[183] X. Liu, P. R. Coxon, M. Peters, B. Hoex, J. M. Cole, and D. J. Fray. Black silicon: fabrication methods, properties and solar energy applications. Energy Environ. Sci., 7:3223-3263, 2014. 
[184] C. Du, C. Gao, G. Yin, M. Chen, and L. Wang. Facile fabrication of a nanoporous silicon electrode with superior stability for lithium ion batteries. Energy Environ. Sci., 4(1037):1037-1042, 2011.

[185] P. van der Linde, Á. Moreno Soto, P. Peñas-López, J. Rodríguez-Rodríguez, D. van der Meer, D. Lohse, J. Huskens, H. Gardeniers, and D. Fernández Rivas. Evolution of electrolytic hydrogen bubbles on microstructured electrodes. In $232^{\text {nd }}$ ECS Meeting, National Harbor, MD, Oct. 1-5, 2017.

[186] H. Vogt. On the supersaturation of gas in the concentration boundary layer of gas evolving electrodes. Electrochim. Acta, 25:527-531, 1980.

[187] P. Peñas-López, Á. Moreno Soto, M. A. Parrales, D. van der Meer, D. Lohse, and J. Rodríguez-Rodríguez. The history effect in bubble growth and dissolution. Part 2. Experiments and simulations of a spherical bubble attached to a horizontal flat plate. J. Fluid Mech., 820:479-510, 2017.

[188] K. Aoki, H. Toda, J. Yamamoto, J. Chen, and T. Nishiumi. Is hydrogen gas in water present as bubbles or hydrated form? J. Electroanal. Chem., 668:83-89, 2012.

[189] K. Kikuchi, S. Nagata, Y. Tanaka, Y. Saihara, and Z. Ogumi. Characteristics of hydrogen nanobubbles in solutions obtained with water electrolysis. J. Electroanal. Chem., 600:303-310, 2007.

[190] H. S. Carslaw and J. C. Jaeger. Conduction of Heat in Solids. Oxford University Press, 1959.

[191] F. Du, D. M. Warsinger, T. I. Urmi, G. P. Thiel, A. Kumar, and J. H. Lienhard V. Sodium hydroxide production from seawater desalination brine: Process design and energy efficiency. Environ. Sci. Technol., 52:5949-5958, 2018.

[192] J. Qi, W. Zhang, and R. Cao. Solar-to-hydrogen energy conversion based on water splitting. Adv. Energy Mat., 8:1701620, 2018.

[193] M. Strasberg. Onset of ultrasonic cavitation in tap water. J. Acoust. Soc. Am., 31:163-176, 1959.

[194] R. E. Apfel. The role of impurities in cavitation-threshold determination. J. Acoust. Soc. Am., 48:1179-1186, 1970.

[195] A. A. Atchley and A. Prosperetti. The crevice model of bubble nucleation. J. Acoust. Soc. Am., 86:1065-1084, 1989. 
[196] A. Luke. Active and potential bubble nucleation sites on different structured heated surfaces. Chem. Eng. Res. Des., 82:462-470, 2004.

[197] H. Vogt. The actual current density of gas-evolving electrodes - notes on the bubble coverage. Electrochim. Acta, 78:183-187, 2012.

[198] W. Botter and O. Teschke. A method for measuring Ohmic resistance of solution layers at gas evolving electrodes. J. Electrochem. Soc., 138:1028-1033, 1991.

[199] P. Scharlin, R. Battino, E. Silla, I. Tunñón, and J. L. Pascual-Ahuir. Solubility of gases in water: Correlation between solubility and the number of water molecules in the first solvation shell. Pure Appl. Chem., 70:1895-1904, 1998.

[200] N. O. Young, J. S. Goldstein, and M. J. Bloack. The motion of bubbles in a vertical temperature gradient. J. Fluid Mech., 6:350-356, 1959.

[201] M. J. Doughty. pH dependent spectral properties of sodium fluorescein ophthalmic solutions revisited. Ophthalmic Physiol. Opt., 30:164-174, 2010.

[202] D. Seo, A. M. Schrader, S-Y. Chen, Y. Kaufman, T. R. Cristiani, S. H. Page, P. H. Koenig, Y. Gizaw, D. W. Lee, and J. N. Israelachvili. Rates of cavity filling by liquids. Proc. Natl. Acad. Sci. U.S.A., 115:8070-8075, 2018. 


\section{Summary}

A better understanding of electrolysis-driven bubble evolution stages is provided: namely the nucleation, growth, and detachment. Superhydrophobic cavities were used to predefine the onset location of electrolytic bubbles on substrates. Using single and multiple superhydrophobic cavities on substrates allowed the elucidation of dissolved gas boundary layer phenomena associated to bubble evolution and the electric potential response caused by detaching electrolytic bubbles in the electrolysis of water. The complex bubble evolution during the electrolysis of water has been compared in experimental and theoretical work with the well-understood evolution of $\mathrm{CO}_{2}$ bubbles in pressure driven supersaturation.

In Chapter 1, an introduction to the thesis is given which expands on the motivation and relevance of the research. Chapter 2 provides a review covering relevant literature on electrolytic bubbles, focusing on the model reaction of water splitting and overpotential losses caused by bubbles, which occur for both resistive, as well as concentration overpotentials.

In Chapter 3, silicon substrates with a superhydrophobic cavity were used in hydrogen bubble evolution during water electrolysis and $\mathrm{CO}_{2}$ bubble evolution during pressure-driven $\mathrm{CO}_{2}$ supersaturation of water. The bubble nucleation times have been measured in both situations as well as the diffusive growth of the bubbles, and the detachment times and sizes. Deviations between the detachment sizes of bubbles formed on the same cavities and theoretical prediction have been thoroughly discussed. The gas transported via detaching bubbles, compared to the total amount of gas generated, has been reported for successive hydrogen bubbles formed during electrolysis. The transport efficiency showed an initial decrease followed by an increase over time; this was attributed to the amount of gas available in the boundary layer adjacent to the evolving bubbles.

In Chapter 4, the theory behind the electrolytically and pressure-driven evolved bubbles described in Chapter 3 is explained in detail. Three regimes occur during the growth of successively formed isolated electrolytic bubbles. First, the depletion regime begins at the start of the electrolysis reaction. The gas boundary layer is depleted of gas during the growth of the bubbles, resulting in the following bubble having less gas available and growing at a slower rate. The second regime occurs after the gas boundary layer near the substrate starts to counteract the depletion, upon 
which bubbles start growing at a faster rate per succession. Finally, a third regime ensues when a quasi-steady state is achieved, originating from the prolonged water splitting reaction homogeneously saturating the bulk liquid surrounding the growing bubble. Comparison of the bubble growth regimes during electrolysis with the well-understood pressure-driven $\mathrm{CO}_{2}$ supersaturation of water shows that for pressure-driven growth, only the depletion regime occurs. The entire solution is supersaturated from the start and the departing bubbles reduce the amount of gas available by transporting gas away from the substrate.

In Chapter 5, ring electrodes surrounding a super hydrophobic cavity were used during the electrolysis of water. With the current density kept smaller than $786 \mathrm{~A} / \mathrm{m}^{2}$, the platinum ring remained free of bubbles and the bubble evolution occurred only on the cavities. The novel electrode design allowed the measurement of a potential drop upon bubble detachment. The potential drop was attributed to the change in proton and gas concentration at the electrode by the movement of liquid induced by departing bubbles.

Chapter 6 presents preliminary experiments on open and closed membraneless electrolyzers systems. The average nucleation times of both hydrogen and oxygen bubbles in the open system electrolyzers, as expected, show that hydrogen bubbles nucleate faster than oxygen bubbles. We attributed the shorter nucleation times to the faster diffusion times of dissolved hydrogen gas in water than the oxygen gas, smaller solubility, and the stoichiometry of the reaction. The preliminary experiments further showed bubble evolution did not occur on the predefined locations in the closed membraneless electrolyzers. Here the superhydrophobic cavities were formed in glass and had a different shape than those formed in silicon, which likely led to wetting of the cavities in glass, preventing the evolution of bubbles on these sites. 


\section{Samenvatting}

In dit proefschrift is een gestreefd naar een betere begripsvorming van door elektrolyse gedreven evolutie van gasbellen. Met name is gekeken naar de verschillende stadia in de bellenproductie, nl. de nucleatie en groei en het loslaten van het oppervlak. Superhydrofobe caviteiten zijn gebruikt om de locatie van elektrolytische belformatie op substraten te definiëren. Het gebruik van een of meer superhydrofobe caviteiten op substraten gaf de mogelijkheid om de opgeloste gasgrenslaagfenomenen geassocieerd aan gasbelevolutie en de elektrische potentiaalrespons, veroorzaakt door loslatende elektrolytische bellen tijdens de elektrolyse van water, op te helderen. De complexe gasbelevolutie tijdens elektrolyse van water is vergeleken in zowel experimenteel werk als theoretisch werk met de goed begrepen nucleatie en evolutie van $\mathrm{CO}_{2}$-bellen in door druk gedreven supersaturatie van water.

In Hoofdstuk 1 is een introductie voor de thesis gegeven die de motivatie en relevantie van het onderzoek onderschrijft. Hoofdstuk 2 biedt een literatuuronderzoek van de relevante literatuur betreffende elektrolytische bellen, gefocust op de modelreactie van watersplitsing en overpotentiaal verliezen veroorzaakt door bellen, welke zich voordoen bij zowel resistieve alsook concentratie overpotentialen.

In Hoofdstuk 3 zijn silicium substraten met een superhydrofobe caviteit gebruikt in waterstofbelevolutie gedurende water elektrolyse en $\mathrm{CO}_{2}$-bel evolutie tijdens druk gedreven oversaturatie van water. De nucleatietijden van de bellen zijn gemeten in beide situaties, evenals de diffusieve groei, het formaat en de verstreken tijd op het moment van het los laten van de bellen. Verschillen tussen de gemeten belgrootte ten tijden van het loslaten van de caviteiten en de theoretische voorspelling zijn breed uitgemeten. Het gastransport via loslatende bellen, vergeleken met de totale hoeveelheid gevormd gas, is gerapporteerd voor waterstofbellen in elektrolyse. De transport efficiëntie liet een initiële afname gevolgd door een toename in tijd zien; dit is toegeschreven aan de hoeveelheid opgelost gas in de grenslaag nabij de evoluerende bellen.

In Hoofdstuk 4 is de achterliggende theorie van de elektrolytische en druk gedreven belevolutie, zoals beschreven in Hoofdstuk 3, in detail beschreven. Drie regimes komen voor tijdens de groei van een opeenvolging van bellen in isolatie. Ten eerste begint een depletieregime aan de start van de elektrolytische reactie. De gasgrenslaag is onttrokken van gas 
gedurende de groei van bellen, erin resulterend dat de volgende bel minder gas ter beschikking heeft en langzamer groeit. Het tweede regime ontstaat zodra de evoluerende gasgrenslaag nabij het substraat het depletie effect tenietdoet, waarop de bellen sneller groeien per successie. Een derde regime begint wanneer een quasi-stabiele staat is bereikt, voortkomende vanuit de voortdurende watersplitsingreactie die het complete volume vloeistof nabij de groeiende bellen homogeen verzadigd. Het vergelijken van bel groei regimes gedurende elektrolyse met het regime tijdens het beter begrepen druk gedreven $\mathrm{CO}_{2}$ verzadigen van water, toonde aan dat voor drukgedreven groei enkel het depletie regime voorkomt. De gehele oplossing is verzadigd vanaf het begin en door de vertrekkende bellen is de gasconcentratie verlaagd met het volume dat door de bellen wordt weggenomen nabij het substraat.

In Hoofdstuk 5 zijn ringelektrodes, die een superhydrofobe caviteit omringen, gebruikt voor de elektrolyse van water. Met stroomdichtheden kleiner dan $786 \mathrm{~A} / \mathrm{m}^{2}$, bleef de platina ring vrij van bellen en vond belevolutie enkel plaats op de caviteiten. Het nieuwe ontwerp creëerde de mogelijkheid om potentiaalverschillen te meten ten tijde van het loslaten van een bel van de caviteit. Het potentiaal verschil is toegeschreven aan de verandering in protonen en gas concentratie nabij de elektrode door de beweging van vloeistof veroorzaakt door de vertrekkende bellen.

Hoofdstuk 6 geeft de voorlopige experimenten in open en gesloten membraamloze elektrolyzers weer. De gemiddelde nucleatietijden van zowel waterstof- als zuurstofbellen in de open elektrolyzer lieten, zoals verwacht, zien dat waterstof bellen sneller nucleëren dan zuurstof bellen. De kortere nucleatietijden zijn toegeschreven aan de mengverhouding van de reactie. De voorlopige experimenten tonen verder dat belevolutie niet plaatsvond op de vooraf gespecificeerde locaties in de gesloten elektrolyzers. Hier waren de superhydrofobe caviteiten gevormd in glas en hadden daardoor een andere vorm dan de caviteiten gevormd in silicium. Dit heeft er mogelijk voor gezorgd dat water makkelijker in de caviteit kon komen een effect dat de bel formatie op deze locaties hinderde. 


\section{Publications and presentations}

\section{Publications}

- Electrolysis-driven and Pressure-controlled Diffusive Growth of Successive Bubbles on Microstructured Surfaces, Langmuir, 33, 2017, 12873-12886. DOI: 10.1021/acs.langmuir.7b02978

- Gas Bubble Evolution on Microstructured Silicon Substrates, Energy Environ. Sci., 11, 2018, 3452-3462 . DOI: 10.1039/C8EE02657B

- Influence of Bubbles on Energy and Mass Transfer Efficiencies of Electrochemical Systems (Chapter 2), in preparation

- Spatial Control over Electrolytic Bubbles Nearby Gas Evolving Electrodes (Chapter 5), in preparation

\section{Presentations}

- MESA+ day 2018 Enschede, The Netherlands

- Oral presentation "Micromachined electrodes for electrolyzers" September 2018

- $232^{\text {nd }}$ ElectroChemical Society Meeting Washington D.C., U.S.A.

- Oral presentation "Evolution of Electrolytic Hydrogen Bubbles on Microstructured Electrodes" October 2017

- MCEC annual meeting 2017 Rhenen, The Netherlands

- Oral presentation "Electrolytic bubbles" and poster presentation, April 2017

- MCEC School 2016 Rhenen, The Netherlands

- Oral presentation "The Epstein-Plesset solution" and poster presentation, October 2016

- MCEC School 2015 Eindhoven, The Netherlands

- Oral presentation "From nanoscale (photo)electrolytic gas generation via microscale bubble nucleation to macroscale bubble transport" and poster presentation, October 2015

\section{Award}

- European travel grant, ElectroChemical Society 



\section{Acknowledgements}

I am not one to use many words on thanking people. A maximum of two words, thank and you, is the most I would typically utter. This is not because I am not thankful but rather that I am at a loss for proper words to express my thanks. However, after four years of $\mathrm{PhD}$, there are many people to thank and I will try to do so in more than two words. Since there are many people to thank, the order in which I will do so is arbitrary. First, I would like to thank both Han and David for providing me the opportunity to work on this $\mathrm{PhD}$ project. I have learned a lot from you both about science, engineering, and in general over the last years. I would like to thank you both for all the patience with me, and for always keeping the overview of the project. In addition, many thanks for introducing me to the right people throughout the project and providing many ideas. I think these ideas were a good counterbalance to all the bears on the different roads I could foresee.

I would like to thank Niels for all the help during student supervision. Your knowledge delivered with great diplomacy resulted in fruitful meetings that I will not likely forget. I would like to thank the members of the MCS group that I have met over the years. I have enjoyed working with this diverse group of people. Having many nationalities represented and various fields of science, resulted in a group with different ambitions and understandings of certain topics. Yet, a combining factor (mostly cake or beer) brought almost all of us together regardless of background. I would like to thank the members of the lunch league for all the enjoyable conversations during the lunch break, and of course, the coffee crew for the early morning coffees during deep (sometimes even scientific) discussions. In general, I would like to thank everyone from MCS for tolerating me and my sometimes-askew sense of humor.

I would like to extend my thanks to all the people that I worked with. The samples I have used throughout the PhD project were not made by me. Rick Elbersen made the first set of samples I ever received; Alexander Milbrat coated a later set (made by Rick) with platinum nano-particles. In addition, Wouter Vijselaar made several batches of samples on which we discovered that the bubbles formed on those samples behaved in a way that we did not observe before, and formed the start of Chapter 5's research. In New York, Zhixiao Zhao made samples for us and had to learn cleanroom techniques from scratch in order to fabricate samples that I later destroyed 
in one afternoon (that is also a part of science I'm afraid). Thanks to Erwin as well, who made samples for one of my students, and of course Stefan who made hundreds of samples for me over the course of the PhD. Many thanks to you all.

When I started out building the first microscope setup, I asked my many questions regarding optics to Carla, who patiently answered them all. I also received great help from Remco in building everything up. I know it must have been a real pain seeing me butcher the setup by just using tape to hold things in place, or using metal strips to raise parts or leveling a carefully crafted custom made holder. Thank you for all the information, ideas, and help. Furthermore, thank you Loreto for letting me borrow a ton of equipment.

I would like to thank all my coauthors for their input, help, and discussions. I would like to express my gratitude to Álvaro and Pablo in particular, two amazing scientists who have bright scientific futures ahead. Thank you for your patience with me.

I would like to thank Miguel Modestino for allowing me to work in his group for several months at the New York University - Tandon school of Engineering. Our weekly meetings during that period and the biweekly meetings afterwards were not only very helpful but also pleasant. In addition, I would like to thank the following people for making my stay in New York a pleasant one; Zhixiao, Adlai, Daniella, Daniel, Myriam, Alyaah, Tana, Cesar, Brian, Nicole, Akash, and Ben. Thank you for making me feel at home so far away from my actual home. With all of you being nice and tolerating my Dutch mindset, I was able to experience the American PhD-lifestyle of working hard and playing hard.

To my family and friends, thanks for keeping things normal. During a $\mathrm{PhD}$, it is easy to get lost in little details; you need a break from it occasionally. Therefore: mom, dad, Ellen, Martijn, Jasper, Kick, Wouter \& Fleur, Erik, Sterre, Mark, Bart, Thijs, Hoon, Dilu, Henk-Willem, Pepijn \& Moniek, and Haye, thank you all for all the dinners, drinks, pub-quizzes, games played, chats, holidays, and support over the years.

Special thanks to Yiyuan, with who I had amazing adventures together. We have seen (from the top of my head) Australia, Belgium, Canada, China, England, Finland, France, Germany, Ireland, Italy, Japan, Luxembourg, Norway, Scotland, South Korea, Sweden, Switzerland, The Netherlands, and The United States of America together in the last couple years. We climbed mountains, swam like sharks, skied, hiked, chased the lights, and ate amazing food. To many more adventures together! 


\section{About the author}

Peter van der Linde was born on the $5^{\text {th }}$ of August 1985 in Maartensdijk, the Netherlands. From an early age Peter was interested in technology and became enthusiastic about high-tech solutions during his bachelor studies. Peter enrolled in a premaster track of the University of Twente on the $1^{\text {st }}$ of September 2011 followed by the master program on the $1^{\text {st }}$ of September 2012. He joined the Mesoscale Chemical Systems group at the University of Twente on the $28^{\text {th }}$ of August 2013 where he worked towards his master degree on his project concerning the development of a micro-device for selective hydrogenation of aromatic hydrocarbons.

On the $1^{\text {st }}$ of January, 2015 he started his $\mathrm{PhD}$ project on electrochemical bubbles at the Mesoscale Chemical Systems group under the supervision of David Fernández Rivas and Han Gardeniers. Leading to the defense of the thesis on the $4^{\text {th }}$ of April 2019.

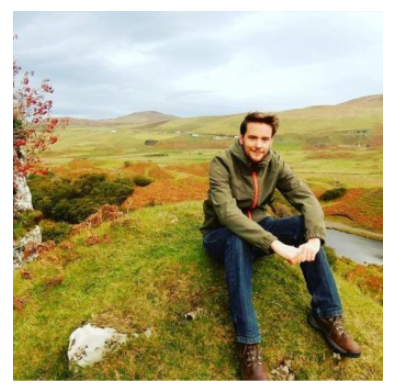

Enschede, The Netherlands February 28, 2019

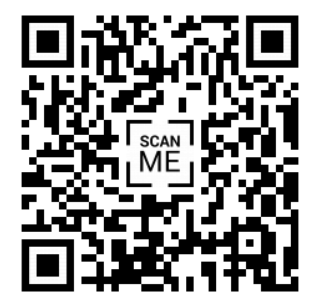


ISBN: $978-90-365-4741-3$ 